\title{
Strangeness Production in Minimum Bias And JET DATA
}

by

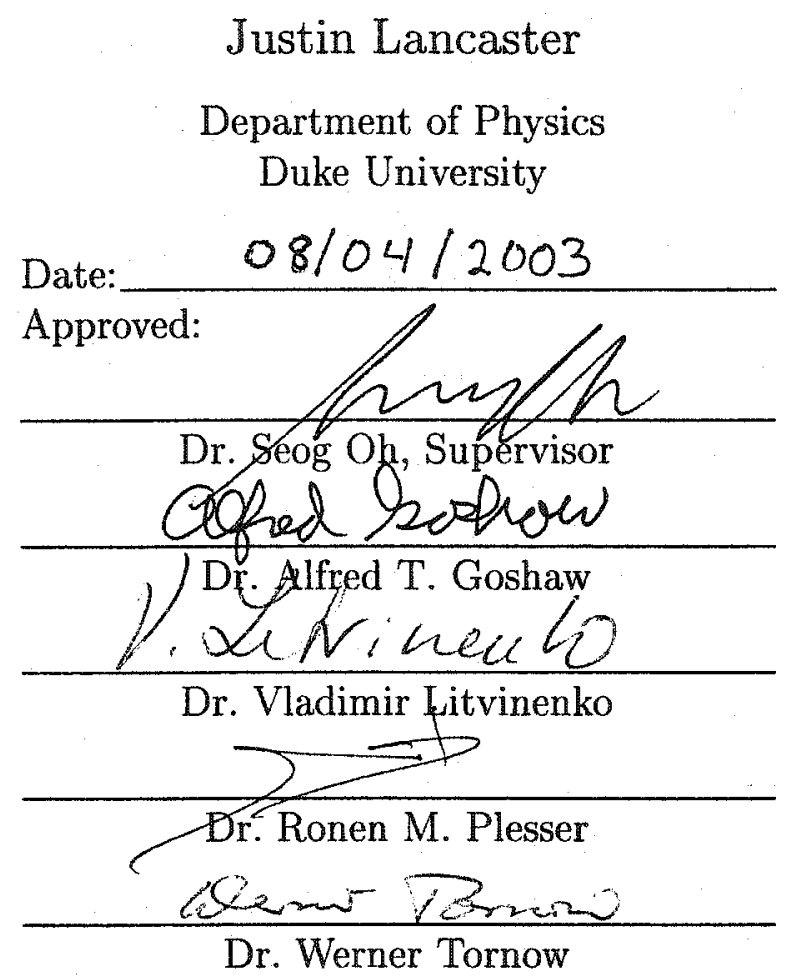

Dissertation submitted in partial fulfillment of the requirements for the degree of Doctor of Philosophy in the Department of Physics in the Graduate School of Duke University 2003 
Copyright (C) 2003 by Justin Lancaster All rights reserved 
ABSTRACT

(Physics)

\section{Strangeness Production in Minimum Bias and Jet DATA}

by

Justin Lancaster

Department of Physics

Duke University

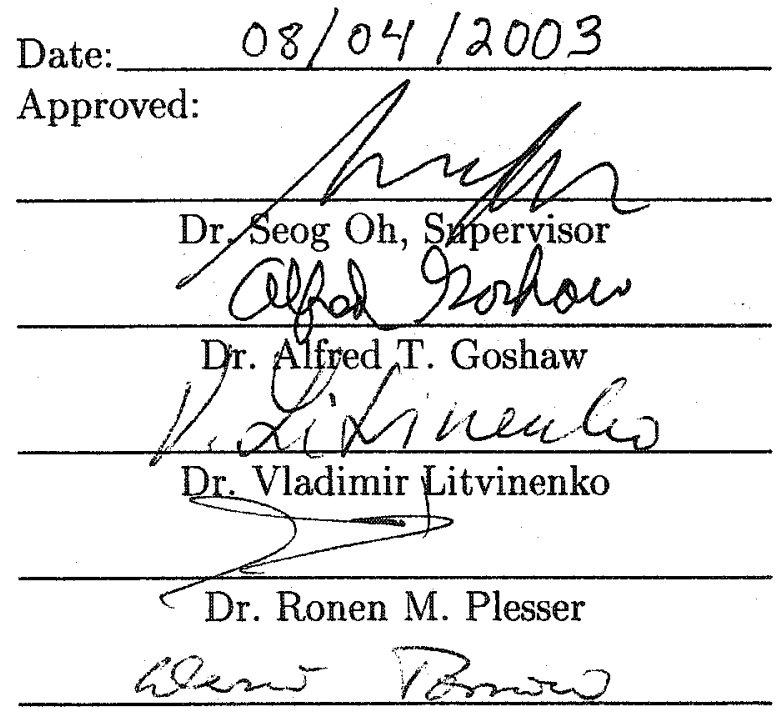

Dr. Werner Tornow

An abstract of a dissertation submitted in partial fulfillment of the requirements for the degree of Doctor of Philosophy in the Department of Physics

in the Graduate School of Duke University

2003 


\begin{abstract}
For the first time, the $K_{S}$ production inside jets originating from $1.8 \mathrm{TeV}$ Tevatron proton-antiproton collisions is researched utilizing the CDF data at Fermilab. Prior to the study of $K_{S}$ production inside jets, the $K_{S}$ production in the Minimum Bias events is examined. The properties of $K_{S}$ production, such as the values of $<p_{T}>, \frac{d N_{K S}}{d \eta}$, lifetime, and invariant cross-section, are found to be consistent with other Minimum Bias publications. After this, the number of $K_{S}$ and tracks inside 0.7 jet cones are computed along with the trigger, background, and efficiency corrections for both the data and the HERWIG+QFL (event generator+detector simulator) Monte Carlo. Furthermore, the fragmentation functions are contrasted with those from the $e^{+} e^{-}$machines.

In the data, the number of $K_{S}$ per jet increases and then reaches a plateau as a function of the jet $E_{T}$. In particular, the number of $K_{S}$ per jet within $1.5<p_{T}<10.0$ $\mathrm{GeV}$ is determined to be $0.156+/-0.007,0.206+/-0.011$, and $0.199+/-0.011$ for the 20-50 GeV, $50-100 \mathrm{GeV}$, and $100-150 \mathrm{GeV}$ jets. Conversely, the number of tracks per jet in the data strictly grows with the jet $E_{T}$, and its values within $1.5<p_{T}<10.0$ $\mathrm{GeV}$ are $2.816+/-0.008,5.107+/-0.009$, and $5.972+/-0.008$ for the $20-50 \mathrm{GeV}, 50-$ $100 \mathrm{GeV}$, and $100-150 \mathrm{GeV}$ cases.

These data results are then compared with those from the HERWIG+QFL Monte Carlo. The Herwig+QFL Monte Carlo results are in agreement to within $10 \%$ as to the number of tracks per jet. Moreover, the number of $K_{S}$ per jet, the data and the Monte Carlo agree to within $5 \%$ for the $20-50 \mathrm{GeV}$ case. However, the HERWIG+QFL Monte Carlo $K_{S}$ per jet values are increasingly above those of the data for $K_{S}$ inside the $50-100 \mathrm{GeV}$ jets (around $20 \%$ too high) and $100-150 \mathrm{GeV}$
\end{abstract}


jets (approximately 35\% to high). We conclude that the HERWIG generates far too many $K_{S}$ inside jets at these higher energy jets. Finally, the fragmentation function is contrasted with equivalently computed quantities from $e^{+} e^{-}$machines, and the results from the Tevatron are below those from $e^{+} e^{-}$machines for all measured jet $E_{T}$ ranges. 


\section{Acknowledgments}

This analysis did not produce itself, nor did $I$, in a vacuum. It is the direct result of thousands of individuals, only a fraction of which I have had the pleasure of meeting. It is also the product of many who through their support, without knowing a quark from a toaster oven, made it possible.

For those who built the accelerator, constructed the detector, gathered the data, wrote the software, developed the field of high energy and physics (especially Galileo), I thank you.

For those always friendly and ever tolerant computer people, who saved at least a computer or two from being tossed out the window, I thank you.

For those graduate students who took time to explain a quark from a toaster oven, or simply that a toaster oven may be hot, I thank you.

For all my mentors at every level, especially my 6th grade teacher, I thank you. For those Duke professors (past and present, in and out of high energy) as well as many other professors digging their cars out of the snow at Fermilab, I thank you. For my committee, who had to read my thesis for a couple of weeks, I thank you. For my advisor, who was not only stuck with me for many years, but had to read my thesis countless times (and this thesis loses its magic after about the 100th read), I thank you.

As for my family and my friends, thank you for your love and support which made this not only possible, but likely. Many thanks.

For the person who is taking his or her time to read this thesis, I thank you. 


\section{Contents}

List of Figures $\quad$ xi

List of Tables $\quad$ xvi

1 Introduction 1

1.1 Quarks and Leptons . . . . . . . . . . . . . . 2

1.2 The fundamental interactions ................... 3

1.2 .1 Electromagnetism . . . . . . . . . . . . 4

1.2 .2 Strong Interaction $\ldots \ldots \ldots 6$

1.2 .3 Weak Interaction . . . . . . . . . . . . 10

1.3 Structure Function . . . . . . . . . . . . . 12

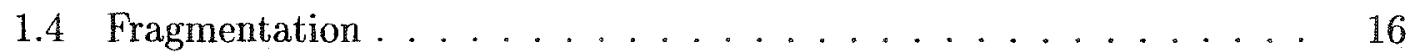

1.5 Hadronization Models . . . . . . . . . . . . . 21

1.6 Motivation of the Analysis ............... 25

2 The Experiment 29

2.1 The Fermilab Tevatron ................... 30

2.2 Collider Detector at Fermilab (CDF) ............ 33

2.2.1 Central Tracking Systems . . . . . . . . . . 37

2.2 .2 Calorimetry .................... 44

2.2 .3 Muon Detector . . . . . . . . . . . . . . 49

vii 
2.2.4 Beam-Beam Counters . . . . . . . . . . 49

2.3 Data Acquisition and Trigger Systems ............ 51

2.3 .1 Level 1 Trigger . . . . . . . . . . . . . 51

2.3 .2 Level 2 Trigger . . . . . . . . . . . . 52

2.3.3 Level 3 Trigger . . . . . . . . . . . . . . 52

2.3 .4 Minimum Bias Triggers . . . . . . . . . . . . 53

2.3 .5 Jet Triggers ...................... 53

3 Minimum Bias: The Selection Criteria 55

3.1 The Event Selection Criteria . . . . . . . . . . . . 56

3.2 The Track Selection Criteria . . . . . . . . . . . . 57

3.3 The Primary Vertex Selection Criteria ... . . . . . . . 59

3.4 The Secondary Vertex Selection Criteria . . . . . . . . . 62

3.5 The $K_{S}$ Selection Criteria . . . . . . . . . . . . 65

4 Minimum Bias: Track Embedding Efficiencies 73

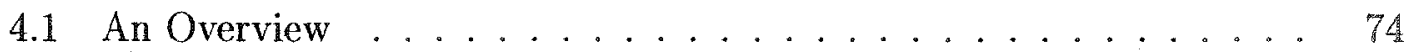

4.2 The Track Embedding Procedure ............ 75

4.3 The $c \tau$ Corrections ................... 82

5 Minimum Bias: Results $\quad 98$

5.1 cr Measurement . . . . . . . . . . . . . . . . 98

5.2 Reconstructed Mass Peak . . . . . . . . . . . . . . . 102

$5.3 p_{T}$ Spectrum . . . . . . . . . . . . . . . 104

5.4 The $\frac{E}{N_{\text {event }}} \frac{d^{3} N_{K S}}{d^{3} p}$ Spectrum ........................... 105

$5.5 \frac{d N_{K S}}{d \eta}$ and $\left\langle p_{T}>\ldots \ldots \ldots \ldots \ldots\right.$ viii 
5.6 Invariant Cross-Section . . . . . . . . . . . . . 108

6 Jets: Selection Criteria 119

6.1 Event Selection . . . . . . . . . . . . . . . . . . . 119

6.1 .1 Cosmic Filter . . . . . . . . . . . . . . . . . . . 119

6.1 .2 Trigger Cut . . . . . . . . . . . . . . 122

6.2 Jet Selection . . . . . . . . . . . . . . . . . . . . 122

6.2 .1 Jet Energy Corrections . . . . . . . . . . . . . . . 124

6.2 .2 Jet Vertex Selection . . . . . . . . . . . . . . . 127

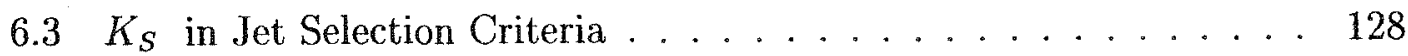

7 Jets: Efficiencies From Track Embedding 141

7.1 Track-embedding Reiterated . . . . . . . . . . . . . . . . . 142

7.2 Track-embedding Jet Procedure . . . . . . . . . . . 146

7.3 Efficiency Dependencies . . . . . . . . . . . . . . . 152

7.4 Comparison with Jets in Data . . . . . . . . . . . . 161

7.5 Single Track Efficiency . . . . . . . . . . . . . 161

8 Jets: Additional Corrections 180

8.1 Applying the $p_{T}$ Efficiency Corrections . . . . . . . 180

8.2 Trigger Corrections to the Data . . . . . . . . . . . 182

8.2 .1 Jet $E_{T}$ Effect $\ldots \ldots \ldots \ldots \ldots \ldots \ldots \ldots$

8.2.2 The Number of Tracks per Jet Effect . . . . . . . . 185

8.3 Background Corrections to the Data $\ldots \ldots \ldots \ldots$

8.4 Results from the Data . . . . . . . . . . . . . . . . . . 192

ix 
9 Jets: HERWIG Monte Carlo

9.1 HERWIG Monte Carlo . . . . . . . . . . . . . . . . . . 203

9.2 "Trigger Corrections" to the Monte Carlo . . . . . . . . . . . 207

9.3 Background Corrections to the Monte Carlo . . . . . . . . . 208

9.4 Comparing the Background of the Data and the HERWIG Monte Carlo210

9.5 Comparing Background with Other Analyses . . . . . . . . . . 211

9.6 The HERWIG Monte Carlo Results . . . . . . . . . . . . . . . . . 213

10 Jets: Results and Conclusions 217

10.1 Correcting for Tracks and $K_{S}$ Inside Jets $\ldots \ldots \ldots \ldots 217$

10.2 Comparing the Data with HERWIG+QFL Monte Carlo . . . . . 219

10.3 Comparing Fragmentation Functions with Other Experiments . . 222

10.4 Estimation of Systematics . . . . . . . . . . . . 224

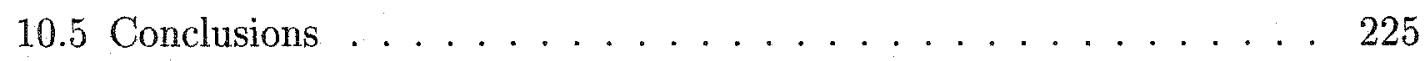

10.6 Overview . . . . . . . . . . . . . . . . . . 2 228

$\begin{array}{ll}\text { Bibliography } & 243\end{array}$

$\begin{array}{ll}\text { Biography } & 249\end{array}$ 


\section{List of Figures}

1.1 The electromagnetic, strong, and weak interaction vertices .... 5

1.2 The running coupling constant $\alpha_{s} \ldots \ldots \ldots 7$

1.3 A hard scattering diagram. . . . . . . . . . . 13

1.4 The structure functions. . . . . . . . . . . . . . 14

1.5 The momentum distributions for the $\mathrm{u}$ and $\mathrm{d}$ quarks. . . . . . 15

1.6 A diagram for the fragmentation for the process $e^{+} e^{-} \rightarrow h X \ldots \ldots 17$

1.7 A pictorial description of jet fragmentation with the three scales $M_{J J}$, $Q_{e f f}$, and $\frac{1}{m_{\pi}} \ldots \ldots \ldots \ldots \ldots$

1.8 The pictorial diagrams of the cluster and the string hadronization models.

2.1 A schematic of the Tevatron accelerator at the Fermi National Accelerator Laboratory. . . . . . . . . . . . . . . . . 30

2.2 The production of antiprotons. . . . . . . . . . 31

2.3 The forward half of the CDF detector. . . . . . . . 33

2.4 A quadrant of the CDF detector. . . . . . . . . . 34

2.5 The diagram of a single SVX barrel. . . . . . . . . . 35

2.6 A figure of a SVX ladder. . . . . . . . . . 36

2.7 A picture of tracks intersecting at a secondary vertex which is displaced $L_{x y}$ from the primary vertex. . . . . . . . . 36

2.8 A figure of a particle leaving behind an ionization trail as it transverses the VTX. . . . . . . . . . . . . . . 39

2.9 A figure of the endplate of the CTC. . . . . . . . . . 41 
2.10 A single wedge of the CEM. . . . . . . . . . . . 43

2.11 A quadrant of the calorimeters showing the $\eta$ - $\phi$ tower segmentation. 44

2.12 A diagram of a quadrant of the PEM. . . . . . . . . . . . 45

2.13 A diagram of one set of Beam-Beam Counters (BBC). . . . . . 50

3.1 The track parameters . . . . . . . . . . . . . . 60

3.2 The $z_{\text {primary }}$ distribution. The cut of $\left|z_{\text {primary }}\right|<60.0 \mathrm{~cm}$ is used. . . 61

3.3 The $\chi^{2}$ distribution and the $\left|z_{K S^{-}} z_{\text {primary }}\right|$ pointback distribution. . 70

3.4 The track $p_{T}$ distribution and the $\eta$ distribution. . . . . . . 71

3.5 The $3 \mathrm{D}$ displacement distribution and the $2 \mathrm{D}$ pointing vector distri-

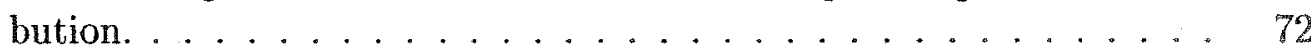

4.1 A transverse view of a Minimum Bias event. A $K_{S}$ has not yet been track embedded. ................ 86

4.2 A transverse view of a Minimum Bias event with a $3.0 \mathrm{GeV}$ trackembedded $K_{S} \ldots \ldots \ldots \ldots \ldots \ldots$

4.3 The $p_{T}$ distribution of $K_{S}$ candidates after track embedding. . . . 88

4.4 The fitted $p_{T}$ distribution of $K_{S}$ candidates after background subtraction. ........................ 89

4.5 The mass distribution of $K_{S}$ candidates after track-embedding. . . 90

4.6 The mass distribution of $K_{S}$ candidates after track-embedding (with and without the $p_{T}$ window cut). ............ 91

4.7 The mass distribution of $K_{S}$ candidates after background subtraction within the $p_{T}$ window. . . . . . . . . . . 92

4.8 The $\chi_{K S}^{2}$ distributions and the $\left|z_{K S^{-}} z_{\text {primary }}\right|$ distributions. . . . . 93

4.9 The daughter track $p_{T}$ distributions and the $\eta^{K S}$ distributions. . . . 94

4.10 The $3 \mathrm{D}$ displacement ${ }^{K S}$ distributions and the $2 \mathrm{D}$ pointing vector distributions. . . . . . . . . . . . . . .

xii 
4.11 The $K_{S}$ efficiency vs. $p_{T}$ curve for the Minimum Bias set. . . . . . 96

4.12 The /slope of the $c \tau$ distribution| vs. $p_{T} \ldots \ldots \ldots 7$

5.1 The $K_{S} \quad c \tau$ spectrum. The curve is obtained after background subtraction and is not corrected for efficiencies. . . . . . . . . 111

5.2 The $K_{S} \quad \mathrm{c} \tau$ spectrum. The curve is obtained after background subtraction and is corrected for efficiencies. . . . . . . . . . 112

5.3 The mass distribution of $K_{S}$ candidates before background subtraction and after Cuts. . .................. 113

5.4 The mass distribution of $K_{S}$ candidates after background subtraction and after cuts. . . . . . . . . . . . . 114

5.5 The $K_{S} \frac{d N}{d p_{T}}$ spectrum. The curve is obtained after background subtraction and is not corrected for efficiencies. . . . . . . 115

5.6 The $\frac{E}{N_{\text {event }}} \frac{d^{3} N_{K S}}{d^{3} p}$ spectrum. The curve is fitted with an exponential. 116

5.7 The $\frac{E}{N_{\text {event }}} \frac{d^{3} N_{K S}}{d^{3} p}$ spectrum. The curve is fitted with a power law from $0.7 \mathrm{GeV}$ to $10.0 \mathrm{GeV} \ldots \ldots \ldots . \ldots . \ldots 117$

5.8 The $\frac{E d^{3} \sigma}{d^{3} p}$ spectrum for $K_{S} \ldots \ldots \ldots \ldots \ldots$

6.1 The fraction of the $K_{S}$ left after each cut in each jet sample. . . . 137

6.2 The jet $E_{T}$ distribution for the Jet20, Jet50, Jet70, and Jet100 sets. 138

6.3 The cosine of the angle distribution and the difference between the jet vertex and primary vertex distribution. . . . . . . . 139

6.4 The mass distribution of $K_{S}$ candidates after background subtraction after cuts. . . . . . . . . . . . . . 140

7.1 A few $\left|\Delta R_{\text {jet-track }}\right|$ distributions normalized to unit area. . . . . 158

7.2 A transverse view of a jet event. A $K_{S}$ has not yet been track



7.3 A transverse view of a jet event with a $3.0 \mathrm{GeV}$ track-embedded $K_{S} \cdot 160$

xiii 
7.4 The mass distribution of $K_{S}$ candidates after track-embedding. . . 163

7.5 The $p_{T}$ distributions of $K_{S}$ candidates after track-embedding. . . . 164

7.6 The $z_{\text {primary }}-z_{\text {jet }}$ distributions and the $z_{\text {jet }}^{r m s}$ distributions. . . . . 165

7.7 The $\chi_{K S}^{2}$ distributions and the $\left|z_{K S^{-}} z_{\text {primary }}\right|$ distributions. . . . 166

7.8 The daughter track $p_{T}$ distributions and the $\eta^{K S}$ distributions. . . 167

7.9 The 3D displacement ${ }^{K S}$ distribution and the $2 \mathrm{D}$ pointing vector distributions. . . . . . . . . . . . . . 168

7.10 The $K_{S}$ efficiency vs. jet $E_{T}$ curve for the 3 groups of $K_{S} p_{T}$ (low,mid,and high). . . . . . . . . . . . . 169

7.11 The $K_{S}$ efficiency vs. $\eta_{j e t}$ curve for the 3 groups of $K_{S} p_{T}$ (low, mid, and high). ..................... 170

7.12 The $K_{S}$ efficiency vs. $\phi_{j e t}$ curve for the 3 groups of $K_{S} p_{T}$ (low,mid,and high).

7.13 The $K_{S}$ efficiency vs. luminosity curve for the 3 groups of $K_{S} p_{T}$ (low,mid,and high). . . . . . . . . . . . 172

7.14 The $K_{S}$ efficiency vs. $\left|z_{\text {primary }}-z_{j e t}\right|$ curve for the 3 groups of $K_{S} p_{T}$

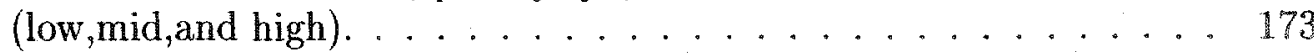

7.15 The $K_{S}$ efficiency vs. the number of tracks per jet curve for the 3 groups of $K_{S} \quad p_{T}$ (low,mid,and high). .......... 174

7.16 The $K_{S}$ efficiency vs. $p_{T}$ curve for $K_{S}$ inside jets. . . . . . 175

7.17 The $K_{S}$ efficiency vs. $p_{T}$ curve for $K_{S}$ inside $20-50 \mathrm{GeV}$ jets. . . 176

7.18 The $K_{S}$ efficiency vs. $p_{T}$ curve for $K_{S}$ inside $50-100 \mathrm{GeV}$ jets. . . 177

7.19 The $K_{S}$ efficiency vs. $p_{T}$ curve for $K_{S}$ inside $100-150 \mathrm{GeV}$ jets. . . 178

7.20 The single track efficiency vs. $p_{T}$ curve for single tracks inside 100$150 \mathrm{GeV}$ jets, $50-100 \mathrm{GeV}$ jets, and $20-50 \mathrm{GeV}$ jets. . . . . . . 179

8.1 The $\frac{1}{N_{j e t}} \frac{1}{p_{T}} \frac{d N_{K S}}{d p_{T}}$ spectra with only $p_{T}$ efficiency and jet $E_{T}$ trigger corrections for $20-50 \mathrm{GeV}, 50-100 \mathrm{GeV}$, and $100-150 \mathrm{GeV}$ jets. . . 195

xiv 
8.2 The $\frac{1}{N_{\text {jet }}} \frac{1}{p_{T}} \frac{d N_{\text {track }}}{d p_{T}}$ spectra with only $p_{T}$ efficiency and jet $E_{T}$ trigger corrections for $20-50 \mathrm{GeV}, 50-100 \mathrm{GeV}$, and $100-150 \mathrm{GeV}$ jets. . . . 196

8.3 The unweighted jet $E_{T}$ for $20-50 \mathrm{GeV}, 50-100 \mathrm{GeV}$, and $100-150 \mathrm{GeV}$ jets. ........................ 197

8.4 The weighted jet $E_{T}$ for $20-50 \mathrm{GeV}, 50-100 \mathrm{GeV}$, and $100-150 \mathrm{GeV}$

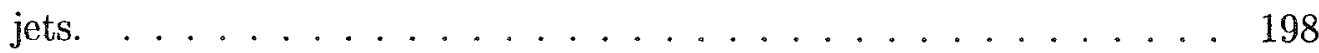

8.5 Another weighted jet $E_{T}$ for $20-50 \mathrm{GeV}, 50-100 \mathrm{GeV}$, and $100-150$ $\mathrm{GeV}$ jets. ...................... 199

8.6 The jet trigger dependence on the number of tracks per jet vs. jet $E_{T} .200$

8.7 The $\frac{1}{N_{j e t}} \frac{1}{p_{T}} \frac{d N_{K S}}{d p_{T}}$ distribution for background $K_{S}$ in jets for data and HERWIG Monte Carlo. . . . . . . . . . . . . . 201

8.8 The $\frac{1}{N_{\text {jet }}} \frac{1}{p_{T}} \frac{d N_{\text {track }}}{d p_{T}}$ distribution for background tracks in jets for data and HERWIG Monte Carlo. . . . . . . . . . . . . . . . . 202

10.1 The $\frac{1}{N_{\text {jet }}} \frac{1}{p_{T}} \frac{d N_{K S}}{d p_{T}}$ spectrum $K_{S}$ inside $20-50 \mathrm{GeV}$ jets. . . . . 233

10.2 The $\frac{1}{N_{\text {jet }}} \frac{1}{p_{T}} \frac{d N_{K S}}{d p_{T}}$ spectrum for $K_{S}$ inside $50-100 \mathrm{GeV}$ jets. . . . 234

10.3 The $\frac{1}{N_{j e t}} \frac{1}{p_{T}} \frac{d N_{K S}}{d p_{T}}$ spectrum for $K_{S}$ inside $100-150 \mathrm{GeV}$ jets. . . . 235

10.4 The $\frac{1}{N_{\text {jet }}} \frac{1}{p_{T}} \frac{d N_{\text {track }}}{d p_{T}}$ spectrum for tracks inside $20-50 \mathrm{GeV}$ jets. . . 236

10.5 The $\frac{1}{N_{\text {jet }}} \frac{1}{p_{T}} \frac{d N_{\text {track }}}{d p_{T}}$ spectrum for tracks inside $50-100 \mathrm{GeV}$ jets. . . 237

10.6 The $\frac{1}{N_{\text {jet }}} \frac{1}{p_{T}} \frac{d N_{\text {track }}}{d p_{T}}$ spectrum for tracks inside $100-150 \mathrm{GeV}$ jets. . 238

10.7 The fragmentation spectrum $K_{S}$ inside $20-50 \mathrm{GeV}$ jets. . . . . . 239

10.8 The fragmentation spectrum $K_{S}$ inside $50-100 \mathrm{GeV}$ jets. . . . . . 240

10.9 The fragmentation spectrum $K_{S}$ inside $100-150 \mathrm{GeV}$ jets. . . . 241

10.10The fragmentation spectrum tracks inside $20-50,50-100,100-150 \mathrm{GeV}$

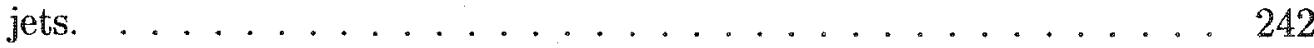




\section{List of Tables}

1.1 The summary of the properties of the leptons .......... 3

1.2 The summary of the properties of the quarks .......... 3

1.3 The summary of the conserved quantities . . . . . . . . . . 4

1.4 The properties of the force carriers ... . . . . . . . . . . 4

1.5 The summary of the quantum numbers for the particles . . . . . . 12

2.1 The summary of the SVX characteristics. . . . . . . . . . 38

2.2 The summary of the calorimeter characteristics . . . . . . . 47

3.1 Minimum Bias set: The number of events after each cut. . . . . 57

3.2 Minimum Bias set: The number of particles after each cut. . . . . . 64

4.1 Minimum Bias set: The $p_{T}$ window ranges for a given $K_{S} \quad p_{T} \ldots \ldots 80$

4.2 Minimum Bias set: The number of $0.7 \mathrm{GeV} K_{S}$ after each cut. . . 80

4.3 Minimum Bias set: The number of $1.6 \mathrm{GeV} K_{S}$ after each cut. . 81

4.4 Minimum Bias set: The number of $3.0 \mathrm{GeV} K_{S}$ after each cut. . . 81

5.1 Minimum Bias set: The $\frac{d N_{K S}}{d \eta}$ and $<p_{T}>$ values for $K_{S} \ldots \ldots \ldots$

5.2 Minimum Bias set: The invariant cross-section comparison . . . . 110

6.1 Jet set: The effect of the cosmic filter on $20-50 \mathrm{GeV}$ jets. . . . . 121

6.2 Jet set: The effect of cosmic filter on $50-100 \mathrm{GeV}$ jets. . . . . . 121

6.3 Jet set: The effect of cosmic filter on greater than $100 \mathrm{GeV}$ jets. . 121

xvi 
6.4 Jet set: The number of events after each cut for all jet samples. . . 130

6.5 Jet set: The number of jets after each cut for Jet20 sample. . . . . 131

6.6 Jet set: The number of jets after each cut for Jet50 sample. . . . . 131

6.7 Jet set: The number of jets after each cut for Jet70 sample. . . . . . 132

6.8 Jet set: The number of jets after each cut for Jet100 sample. . . . . 132

6.9 Jet set: The number of $K_{S}$ after each cut for all jet samples. . . . 133

6.10 Jet set: The number of $K_{S}$ in $20-50 \mathrm{GeV}$ jets after each cut. . . . 134

6.11 Jet set: The number of $K_{S}$ in $50-100 \mathrm{GeV}$ jets after each cut. . . 135

6.12 Jet set: The number of $K_{S}$ in $100-150 \mathrm{GeV}$ jets after each cut. . . 136

7.1 Jet set: The groups of embedded $K_{S}$ in jets. . . . . . . . 154

7.2 Jet set: The $p_{T}$ window ranges for a given $K_{S} p_{T} \ldots \ldots \ldots 154$

7.3 Jet set: The number of $1.6 \mathrm{GeV} K_{S}$ in $20-50 \mathrm{GeV}$ jets after cuts. . 155

7.4 Jet set: The number of $1.6 \mathrm{GeV} K_{S}$ in $50-100 \mathrm{GeV}$ jets after cuts. 155

7.5 Jet set: The number of $1.6 \mathrm{GeV} K_{S}$ in $100-150 \mathrm{GeV}$ jets after cuts. 156

7.6 Jet set: The number of $6.0 \mathrm{GeV} K_{S}$ in $20-50 \mathrm{GeV}$ jets after cuts. . 156

7.7 Jet set: The number of $6.0 \mathrm{GeV} K_{S}$ in $50-100 \mathrm{GeV}$ Jets After Cuts. 157

7.8 Jet set: The number of $6.0 \mathrm{GeV} K_{S}$ in $100-150 \mathrm{GeV}$ jets after cuts. 157

8.1 Jet set: The number of $K_{S}$ per jet in the data. . . . . . . 193

8.2 Jet set: The number of tracks per jet in the data. . . . . . . . 193

8.3 Jet set: $\left(2^{*}\right.$ The number of $\left.K_{S}\right)$ per track in the data. . . . . . 193

9.1 Jet set: The number of $K_{S}$ per jet in the Monte Carlo data. . . . 216

9.2 Jet set: The number of tracks per jet in Monte Carlo data. . . . . . 216

9.3 Jet set: ( $2 *$ The number of $K_{S}$ ) per track in Monte Carlo data. . . 216 
10.1 Jet set: The $\frac{N_{\text {particle }}}{j e t}$ and $\left\langle p_{T}\right\rangle$ values for tracks and $K_{S}$ in $20-50$ GeV Jets in the data. . . . . . . . . . . . . . . . . 230

10.2 Jet set: The $\frac{N_{\text {particle }}}{\text { jet }}$ and $\left\langle p_{T}\right\rangle$ values for tracks and $K_{S}$ in $20-50$ $\mathrm{GeV}$ jets in HERWIG Monte Carlo. . . . . . . . . . . . . . . . 230

10.3 Jet set: The $\frac{N_{\text {particle }}}{j e t}$ and $\left\langle p_{T}\right\rangle$ values for tracks and $K_{S}$ in $50-100$ GeV jets in the data. . . . . . . . . . . . . . . 231

10.4 Jet set: The $\frac{N_{\text {particle }}}{\text { jet }}$ and $\left\langle p_{T}\right\rangle$ values for tracks and $K_{S}$ in $50-100$ $\mathrm{GeV}$ jets in HERWIG Monte Carlo. . . . . . . . . . . . . . . 231

10.5 Jet set: The $\frac{N_{\text {particle }}}{j e t}$ and $\left\langle p_{T}\right\rangle$ values for tracks and $K_{S}$ in 100-150 $\mathrm{GeV}$ jets in the data. . . . . . . . . . . . . . . . 232

10.6 Jet set: The $\frac{N_{\text {particle }}}{\text { jet }}$ and $\left\langle p_{T}\right\rangle$ values for tracks and $K_{S}$ in $100-150$ $\mathrm{GeV}$ jets in HERWIG Monte Carlo. . . . . . . . . . . . . . 232 


\section{Chapter 1}

\section{Introduction}

"The finder of a new particle used to rewarded by a Nobel Prize, but such a discovery now ought to be punished by a $\$ 10,000$ fine," said Nobel Prize winner Willis Lamb in his 1955 acceptance speech. Lamb is referring the discovery of a new class of unexpected and unpredicted "strange" particles that appeared to drop from the heavens (in this case, they actually did in the form of the earliest and still the highest energy accelerators, cosmic rays). It sent shockwaves throughout the particle physics community. "Strange" particles are produced only in pairs very quickly $\left(\sim 10^{-23} \mathrm{sec}\right)$ and decay by themselves slowly $\left(\sim 10^{-10} \mathrm{sec}\right)$. Rochester and Butler [1] first discovered the $K_{S} \rightarrow \pi^{+} \pi^{-}$in 1947, Powell [2] found the $K_{+} \rightarrow \pi^{+} \pi^{+} \pi^{-}$in 1949, and Anderson discovered $\Lambda^{0} \rightarrow \pi^{-} p^{+}$in 1950, and $\eta, \phi, \Omega, \Sigma, \Xi$, and many other "strange" particles have been shown to exist. Today, my analysis (along with other analyses) depends on $K_{S}$ as the starting point because they are produced so abundantly. In fact, my study looks at the production of $K_{S}$ in objects called jets created from fragmenting quarks and gluons. Yet, at one time, as recently as 60 years ago, no one could conceive of such an analysis nor the tool necessary for its realization [3].

We will begin a very brief introduction to a field, fundamental in nature, grand in scale, precisely explaining so much and with many questions unanswered. A 
discussion of the constituents of matter and how they are related to the 4 forces, the running coupling constant, parton distribution functions, and fragmentation will all be put forth in this chapter.

\subsection{Quarks and Leptons}

There are 3 generations of fractionally charged fermions called quarks (up,down), (strange,charm), (bottom,top), and 3 generations of charged and neutral fermions called leptons $\left(e, \nu_{e}\right),\left(\mu, \nu_{\mu}\right)$, and $\left(\tau, \nu_{\tau}\right)[4]$. The $\tau$ and $\mu$ particles are similar to electrons, except more massive. On the other hand, the neutrinos are extremely light neutral particles with masses of at most $20 \mathrm{MeV}$. The neutrinos regularly travel through earth without interacting with a single particle.

Through mediators, quarks and leptons interact via gravitational, electromagnetic, strong, or weak forces. Leptons do not interact through the strong force and conserve a quantity called lepton number in all interactions they participate in with the exception of "neutrino oscillation" experiments where neutrinos are believed to change flavor.

Quarks are unable to exist alone in nature. In fact, quarks and antiquarks can only exist as either quark-antiquark pairs, referred to as mesons, or 3 quark (antiquark) combinations called baryons (antibaryons). Consequently, the resulting charge is an integer. $K_{S}$ and $\phi$ are examples of mesons, and $\Lambda^{0}$ and $\bar{\Lambda}^{0}$ are examples of baryons. Quarks also conserve a different set of quantum numbers called "truth", "beauty", "charm", and "strangeness" in all but weak interactions [3] [5] [6] [7] [8]. 
Table 1.1: The summary of the properties of the leptons: S(spin), $L_{e}$ (lepton electron number), $L_{\mu}$ (lepton muon number), $L_{\tau}$ (lepton tau number), $Q$ (electric charge) $\left[1.6 \times 10^{-19} C\right], m_{0}$ (mass), AP(anti-particle) [8]

\begin{tabular}{|l|l|rrrrrrr|r|}
\hline & & Spin & $L_{e}$ & $L_{\mu}$ & $L_{\tau}$ & $Q[e]$ & $m_{0}[\mathrm{MeV}][9]$ & $\mathrm{AP}$ \\
\hline \hline \multirow{3}{*}{ 1st generation } & $e^{-}$ & $1 / 2$ & 1 & 0 & 0 & -1 & $0.510998902 \pm 0.000000021$ & $e^{+}$ \\
& $\nu_{e}$ & $1 / 2$ & 1 & 0 & 0 & 0 & $<15 \times 10^{-6}$ & $\bar{\nu}_{e}$ \\
\hline \multirow{3}{*}{ 2nd generation } & $\mu^{-}$ & $1 / 2$ & 0 & 1 & 0 & -1 & $105.658357 \pm 0.000005$ & $\mu^{+}$ \\
& $\nu_{\mu}$ & $1 / 2$ & 0 & 1 & 0 & 0 & $<0.17$ & $\bar{\nu}_{\mu}$ \\
\hline \multirow{3}{*}{ 3rd generation } & $\tau^{-}$ & $1 / 2$ & 0 & 0 & 1 & -1 & $1777.03 \pm 0.3$ & $\tau^{+}$ \\
& $\nu_{\tau}$ & $1 / 2$ & 0 & 0 & 1 & 0 & $<18.2$ & $\bar{\nu}_{\tau}$ \\
\hline
\end{tabular}

Table 1.2: The summary of the properties of the quarks: S(spin), B(baryon number), I(strong isospin), $s$ (strangeness), $c$ (charm), $b$ (beauty), $t$ (truth), $Q$ (electric charge) $\left[1.6 \times 10^{-19} C\right], m_{0}$ (mass), AP(anti-particle). The particle properties in the Standard Model for the electroweak interaction. The quantum numbers of weak isospin $\mathrm{T}$, and its projection, $T_{3}$, weak hypercharge, $\mathrm{Y}$, and the electric charge, $\mathrm{Q}$, are given $\left(Q=T_{3}+\frac{1}{2} Y\right)$. The right-handed fermions (labeled by the index $\mathrm{R}$ ) are weak-isospin singlets $(T=0)$, while the left-handed fermions (labeled $\mathrm{L}$ ) are weakisospin doublets $(\mathrm{T}=1 / 2)$. Massless neutrinos appear only as left-handed particles and right-handed antiparticles. The $Z^{0}$ and the photon have the same quantum numbers $\left(T_{3}=Y=Q=0\right)$ and can therefore mix. The gluons have $T=Q=Y=0$ and therefore do not interact in the electroweak interactions [8] [10].

\begin{tabular}{|l|c|rcccccccr|c|}
\hline & & Spin & $\mathrm{B}$ & $\mathrm{I}$ & $s$ & $c$ & $b$ & $t$ & $Q[e]$ & $m_{0}[\mathrm{MeV}][9]$ & $\mathrm{AP}$ \\
\hline \hline lst & $u$ & $1 / 2$ & $1 / 3$ & $1 / 2$ & 0 & 0 & 0 & 0 & $+2 / 3$ & $1.5-5$ & $\bar{u}$ \\
generation & $d$ & $1 / 2$ & $1 / 3$ & $1 / 2$ & 0 & 0 & 0 & 0 & $-1 / 3$ & $3-9$ & $\bar{d}$ \\
\hline 2nd & $c$ & $1 / 2$ & $1 / 3$ & 0 & 0 & 1 & 0 & 0 & $+2 / 3$ & $1.1 \times 10^{3}-1.4 \times 10^{3}$ & $\bar{c}$ \\
generation & $s$ & $1 / 2$ & $1 / 3$ & 0 & -1 & 0 & 0 & 0 & $-1 / 3$ & $60-170$ & $\bar{s}$ \\
\hline 3rd & $t$ & $1 / 2$ & $1 / 3$ & 0 & 0 & 0 & 0 & 1 & $+2 / 3$ & $173.8 \times 10^{3} \pm 5.2 \times 10^{3}$ & $\bar{t}$ \\
generation & $b$ & $1 / 2$ & $1 / 3$ & 0 & 0 & 0 & -1 & 0 & $-1 / 3$ & $4.1 \times 10^{3}-4.4 \times 10^{3}$ & $\bar{b}$ \\
\hline
\end{tabular}

\subsection{The fundamental interactions}

There are 4 fundamental interactions in nature: gravity, electromagnetic, strong, and weak. Each forces incorporates a mediator that governs how quarks and leptons 
Table 1.3: Summary of the conserved quantities in the strong, electromagnetic and weak interactions [8] [9]

\begin{tabular}{|l|c|c|c|}
\hline Conserved Quantity & Strong & Electromagnetic & Weak \\
\hline I(Isospin) & Yes & No & No $(\Delta \mathrm{I}=1$ or $1 / 2)$ \\
S(strangeness) & Yes & Yes & No $(\Delta \mathrm{S}=1,0)$ \\
C(charm) & Yes & Yes & No $(\Delta \mathrm{C}=1,0)$ \\
P(parity) & Yes & Yes & No \\
C(charge-conjugation parity) & Yes & Yes & No \\
CP(or T) & Yes & Yes & Yes \\
& & & (except $K^{0}$ and $\left.B^{0}\right)$ \\
CPT & Yes & Yes & Yes \\
\hline
\end{tabular}

interact. The mediators for the electromagnetic, strong, and weak forces are the photon, gluon, and $W / Z$ Bosons, respectively. Gravity has yet to be incorporated in this fundamental framework because it so much weaker than the other forces. The mediator of the gravitational force, the "graviton" has not been discovered either directly or indirectly [3] [5] [6] [7] [8].

Table 1.4: Force Carriers: S(spin), $Q$ (electric charge), $m_{0}$ (mass) [8]

\begin{tabular}{|c|ccc|}
\hline & Spin & $Q[e]$ & $m_{0}[\mathrm{MeV}][9]$ \\
\hline \hline$\gamma$ & 1 & 0 & $<2 \times 10^{-22}$ \\
$\mathrm{~g}$ & 1 & 0 & 0 (assumed) \\
$W^{ \pm}$ & 1 & \pm 1 & $80419 \pm 56$ \\
$Z$ & 1 & 0 & $91182 \pm 2.2$ \\
\hline
\end{tabular}

\subsubsection{Electromagnetism}

Classical electromagnetism is connected to quantum field theory to yield what is considered the most successful and precise theory of all time: Quantum Electrodynamics (QED). QED has the charged particles interacting through means of ex- 


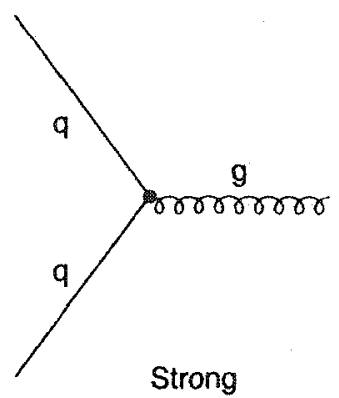

Strong
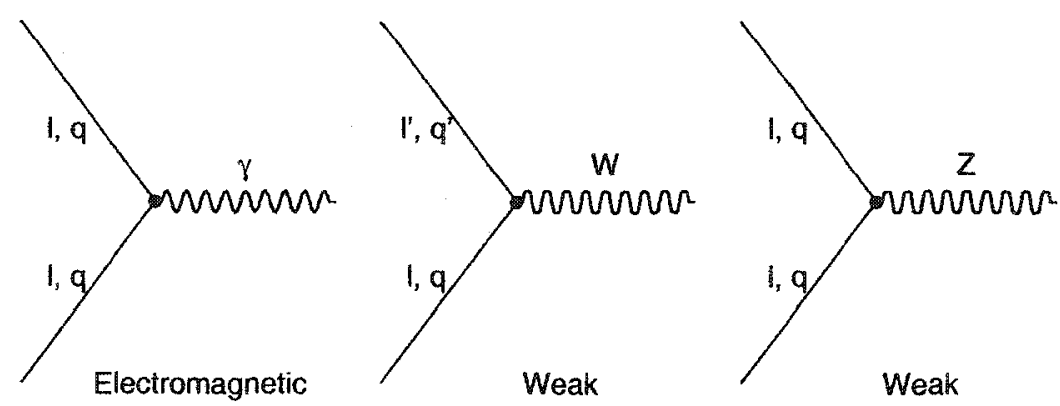

Figure 1.1: The electromagnetic, strong, and weak interaction vertices [5]

changing a field quanta called photons such that charge is conserved. So accurate is QED that the magnetic momentum of an electron is calculated to at least 10 decimal places. In addition, branching ratios and cross-sections are able to be extracted with the aid of Feynman diagrams. Feynman diagrams and charge screening will be briefly touched upon.

Feynman diagrams correlate pictures of lines and vertices to mathematical terms necessary for QED calculations. For every calculation, there are an infinite number of loops, lines, and vertices; however, QED is perturbative so the results converge because higher order terms get smaller and smaller. This is case because every vertex is associated with a factor of the electromagnetic coupling factor, $\sqrt{4 \pi \alpha}$, between photons and charged particles. In particular, $\alpha$ is proportional to the square of the electron charge, and $\alpha$ is equal to the fine structure constant, $\alpha \approx \frac{1}{137}$ at low $Q^{2}$. So for a given cross-section calculation, each successive term. with higher orders of $\alpha$, is less significant than the preceding one.

Aside from setting the method of mathematical formulation, QED also predicts the spontaneous production of virtual $e^{+} e^{-}$pairs that leads to charge screening. By incorporating the uncertainty principle, conservation of energy is violated for a 
brief time, $\Delta E \Delta t \sim \not h$ where $\Delta E$ is the unconserved energy used to produce $e^{+} e^{-}$ virtual pairs. Hence, an electron can surround itself by a cloud of virtual $e^{t} e^{-}$ pairs by emitting virtual photons that emit virtual $e^{+} e^{-}$pairs with the positrons through electromagnetic interactions tending to be closer to the "bare" electron. One consequence is a smaller measured charge of an electron because of the charge screening of the cloud, and the effective charge of an electron increases as one moves through the cloud. The reduction of the effective charge due to charge screening tends to reduce the electromagnetic coupling factor. The fine structure constant is defined as the measurement of the electromagnetic coupling factor at infinity, and the electromagnetic coupling factor $\alpha$ asymptotically increases with energy as less charge is screened. Higher energy interactions have stronger electromagnetic coupling factors in Feynman diagrams [3] [5] [6] [7] [8].

\subsubsection{Strong Interaction}

The strong force is responsible for combining quarks into hadrons as well as binding neutrons and protons. The strong force behaves very similarly to QED in the manner that a charge is conserved and there is a quantized mediator present in every strong interaction, and a theory called Quantum Chromodynamics explains these types of interactions as well as empirical facts that Quantum Electrodynamics is unable to do. The questions as to why there are not any free stable quarks in nature, to why quarks combine only certain combinations of anti-quark quark pairs or in triplets of integer charge, to why the Pauli Principle appears to be violated in case of $\Delta^{++}$, and to why the quark production rate is 3 times that of muon production rate in electron annihilation processes are all adequately addressed with Quantum Chromodynamics. 


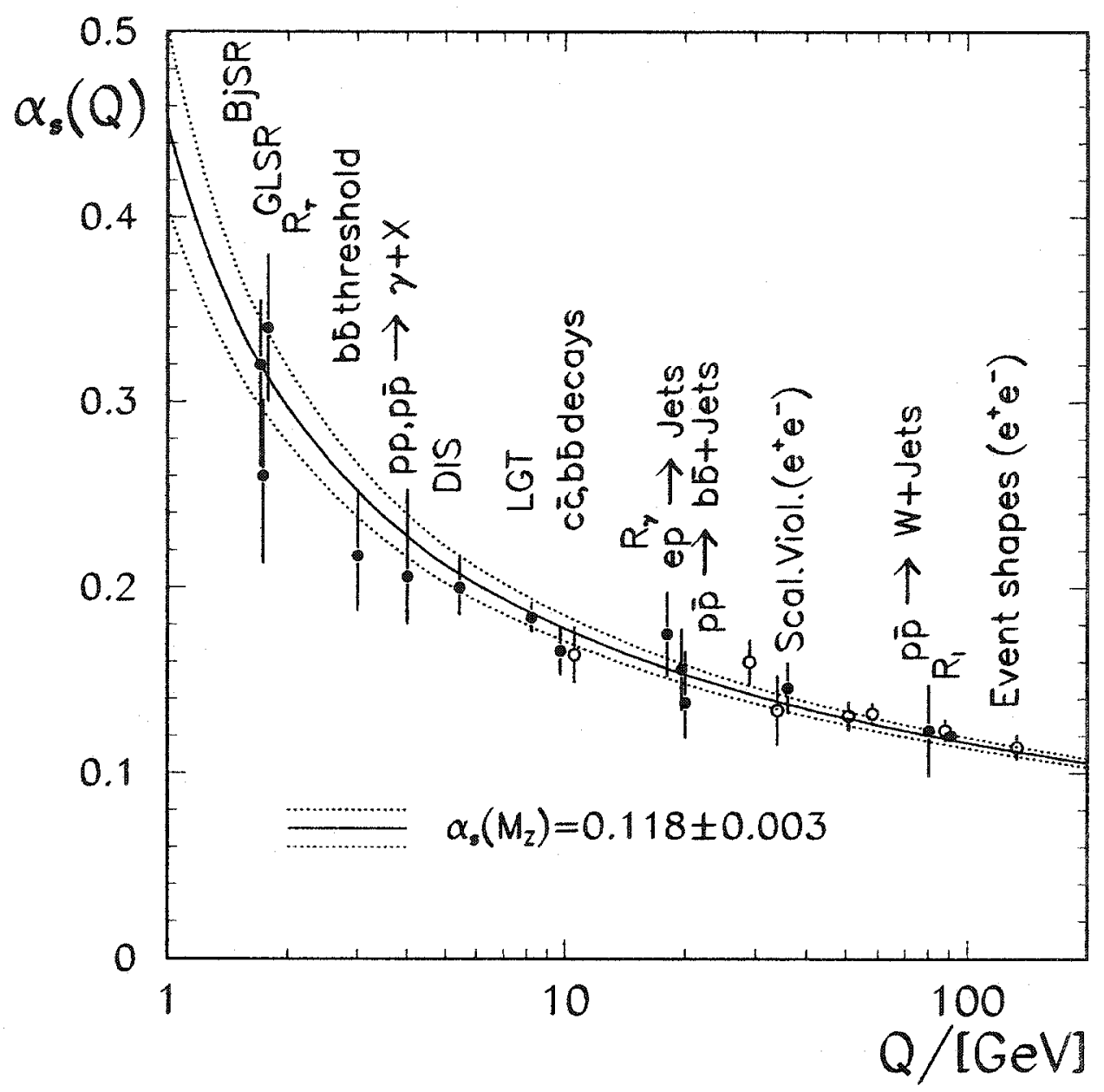

Figure 1.2: The running coupling constant $\alpha_{s}[11]$.

The nature of the strong force is complex, and it is given by equation 1.1 [12],

$$
V(r)=-\frac{4}{3} \frac{\alpha_{s}}{r}+k r
$$

where $\alpha_{s}$ is the running coupling constant, $r$ is the distance between quarks, $k$ is constant approximately equal to $1.0 \mathrm{GeV} \mathrm{fm}^{-1}$, and $V(r)$ is the potential between the two quarks. There are essential two separate components: the first term dominates at small interactions distances (or high momentum transfer scales) and the 
second term dominates at large interactions distances (or low momentum transfer scales). The short-range term is similar in form to the electromagnetic point potential except that the $\alpha_{s}$ term is not constant, hence the term "running", but is a function of momentum transfer. The lowest order term of the $\alpha_{s}$ is given by the equation 1.2 (shown in Figure 1.2), where $Q^{2}$ is the square of the four-momentum transfer, $n_{f}$ is the number of quarks allowed by energy of the interaction, and $\Lambda_{Q C D}$ is the approximately $200 \mathrm{MeV}$ [13] (approximately the energy boundary between free quarks and hadrons) [12]:

$$
\alpha_{s}\left(\left|Q^{2}\right|\right)=\frac{12 \pi}{\left(33-2 n_{f}\right) \ln \left(\left|Q^{2}\right| / \Lambda_{Q C D}^{2}\right)}
$$

The interpretation of these equations will now be highlighted. At large momentum transfer scales (short distances), the first term dominates. $\alpha_{s}$ decreases with energy to about $0.1, Q^{2} \gg \Lambda_{Q C D}^{2}$, such that perturbative QCD can be applied and quarks and gluons interact with a small force. As the momentum transfer scale increases, observed charge decreases to that of just the bare value. This reduction of the strong force is known as "asymptotic freedom", and such hard scattering processes describe such high energy transfer events where higher order $\alpha_{s}$ terms have smaller and smaller effects.

At large distances dominated by the linear term of the strong force, the potential is much stronger, to the point that free quarks are more energetically likely to create quark-antiquark pairs to reduce the separation of the charges and these quarks combine to form numerous hadronic bound states. As a result, isolated quarks have not been observed in nature. This point is one of the most interesting aspects in my opinion about particle physics that the quark, a fundamental quantity of matter, have never been discovered in isolation. 
The experimental evidence of the existence of color includes the existence of the $\Delta^{++}$(uuu) particle in its lowest orbital momentum state $(l=0)$ with its spins aligned $(J=\mathrm{S}=3 / 2)$. This state has a symmetric wavefunction (without a color wavefunction) which violates the Pauli Exclusion Principle. However, with the introduction of color wavefunction in addition to the spatial and spin wavefunctions, the overall state is still anti-symmetric, honoring the Pauli Exclusion Principle.

Furthermore, only colorless bound states of quark-antiquark pairs (mesons) and three quark combinations (baryons) have been found experimentally. Although theoretically possible for there to be other combinations of quarks (i.e. pentaquarks), none have been discovered. Moreover, the final bound states have integer charges. In addition, the octet of color-carrying gluon mediators can couple together (photons do not have this property in QED) which gives rise to the linear term of the strong interaction potential. All of these effects have been observed.

Another strong piece of evidence of color is the ratio of quark-antiquark production and muon pair production rates from electron-positron experiments. This ratio is equal to the product of the number of colors and the sum of the square of the quark charges. Since the number of flavors available is contingent on the center of mass energy of the experiment, only the quark charges kinematically allowed at the center-of-mass energy are included. The best agreement with the data occurs when the number of colors is equal to three (i.e. non-zero).

At last, for low momentum transfer interactions where perturbative QCD breaks down, individual quarks and gluons are transformed into hadrons during fragmentation processes. These clusters of bound hadrons should conserve the momenta and energy of the initial parton. Furthermore, so-called fragmentation is completely are believed to depend only upon the energy and type of the outgoing parton rather 
than the particular process which generated them. Various fragmentation models are used to describe these soft QCD processes. Experimentally, 2 jet processes correspond to 2 clusters of hadrons from 2 quarks. At higher energies, there exists 3 jet processes of which 2 are jets are due to quarks and the third jet is due to a gluon. These 3 jet events, where one jet is a gluon jet, is testimony to the existence of gluons as mediators [3] [5] [6] [7] [8].

\subsubsection{Weak Interaction}

Electroweak interaction unifies both the weak and the electromagnetic interactions into a single theory [14]. The weak force differs from the electromagnetic force in many respects, and the weak force is necessary to explain $\beta$ decay (protons turn into neutrons and visa versa) along with other processes that allow particles of one type to be transformed into another. The experimental evidence is overwhelming, including confirming the existence of 3 massive bosonic mediators in 1983 at CERN SPS with $p \bar{p}$ collisions. Other predictions such as the infamous Higgs particle, responsible for giving mass to the bosonic mediators (as well as other particles), remain to be confirmed.

Unlike QED, the mediators which interact weakly with particles have mass. The mediators include $W^{+}, W^{-}$, and $Z^{0}$ particles where the mass of the $W$ boson is measured to be $81 \pm 5 \mathrm{GeV} / \mathrm{c}^{2}[15]$ and $80_{-6}^{+10} \mathrm{GeV} / \mathrm{c}^{2}[16]$ by UA1 and UA2 (CERN experiments), respectively. The mass of the $Z$ boson is measured to be $95.2 \pm 2.5 \mathrm{GeV} / \mathrm{c}^{2}[17]$ and $91.9 \pm 1.3 \pm 1.4 \mathrm{GeV} / \mathrm{c}^{2}[18]$ by $\mathrm{UA} 1$ and $\mathrm{UA} 2$, respectively. The current accepted mass measurements are shown in Table 1.4. These massive mediators give rise to weak force interactions which have a range of around $\sim 10^{-3}$ fm. 
The weak force also couples to particles differently. Parity-violating and chargeconjugation violating processes are permitted in weak interactions. Also, the weak force also conserves quantities called weak isospin and weak hypercharge $\left(Q=T_{3}+\frac{Y}{2}\right.$ where $Y$ is the hypercharge and $T_{3}$ is the $3 \mathrm{rd}$ component of the weak isospin) that distinguish between right-handed and left-handed fermions.

$W^{ \pm}$bosons not only change the charge of fermions by \pm 1 but also the flavor of the fermions. These processes are called "flavor-changing charged currents". As a result, the weak force allows members of similar or different generations to interact leptonically $\left(\mu^{-} \rightarrow e^{-}+\overline{\nu_{e}}++\nu_{\mu}\right)$, hadronically $\left(\Lambda^{0} \rightarrow \pi^{-}+p\right)$, or semi-leptonically $\left(n \rightarrow p+e^{-}+\overline{\nu_{e}}\right)$.

On the other hand, neutral $Z$ bosons in "neutral current" processes may change the flavor but not the charge of the interacting particles. Furthermore, particles of similar or different generations may interact. The process $\nu_{e}+e^{-} \rightarrow \nu_{e}+e^{-}$is an example of similar generations interacting weakly, and the process $e^{+}+e^{-} \rightarrow q+\bar{q}$ is an example of different generations interacting weakly. Notice that through $W$ and $Z$ bosons (unlike photon mediators of the electromagnetic force), neutrinos also interact [3] [5] [6] [7] [8].

The weak force coupling constant $(g)$ is proportional to the electromagnetic coupling constant $\left(g^{\prime}\right)$ by the weak mixing angle (Weinberg angle $\theta_{W}$ ) in equation 1.3 , and the Weinberg angle is related to the masses of the $W$ and $Z$ bosons according the relation given by equation 1.4 .

$$
\begin{gathered}
\tan \theta_{W}=\frac{g^{\prime}}{g} \\
\cos \theta_{W}=\frac{M_{W}}{M_{Z}}
\end{gathered}
$$


Table 1.5: The summary of the quantum numbers for the particles [8] [10]

\begin{tabular}{|lcccc|}
\hline Quarks & $T$ & $T_{3}$ & $\mathrm{Q}$ & $\mathrm{Y}$ \\
\hline \hline$u_{L}, c_{L}, t_{L}$ & $1 / 2$ & $+1 / 2$ & $+2 / 3$ & $+1 / 3$ \\
$d_{L}, s_{L}, b_{L}$ & $1 / 2$ & $-1 / 2$ & $-1 / 3$ & $+1 / 3$ \\
\hline$u_{R}, c_{R}, t_{R}$ & 0 & 0 & $+2 / 3$ & $+4 / 3$ \\
$d_{R}, s_{R}, b_{R}$ & 0 & 0 & $-1 / 3$ & $-2 / 3$ \\
\hline \hline $\bar{u}_{R}, \overline{c_{R}}, \overline{t_{R}}$ & $1 / 2$ & $-1 / 2$ & $-2 / 3$ & $-1 / 3$ \\
$\overline{d_{R}}, \overline{s_{R}},{\overline{b_{R}}}_{R}$ & $1 / 2$ & $+1 / 2$ & $+1 / 3$ & $-1 / 3$ \\
\hline$\overline{u_{R}}, \overline{c_{R}}, \overline{t_{R}}$ & 0 & 0 & $-2 / 3$ & $-4 / 3$ \\
$\overline{d_{R}}, \bar{s}_{R}, \overline{b_{R}}$ & 0 & 0 & $+1 / 3$ & $+2 / 3$ \\
\hline \hline Leptons & $T$ & $T_{3}$ & $\mathrm{Q}$ & $\mathrm{Y}$ \\
\hline \hline$\nu_{e}, \nu_{\mu}, \nu_{\tau}$ & $1 / 2$ & $+1 / 2$ & 0 & -1 \\
$e_{L}^{-}, \mu_{L}^{-}, \tau_{L}^{-}$ & $1 / 2$ & $-1 / 2$ & -1 & -1 \\
$e_{R}^{-}, \mu_{R}^{-}, \tau_{R}^{-}$ & 0 & 0 & -1 & -2 \\
\hline \hline$\overline{\nu_{e}}, \overline{\nu_{\mu}} \overline{\nu_{\tau}}$ & $1 / 2$ & $-1 / 2$ & 0 & +1 \\
$e_{R}^{+}, \mu_{R}^{+}, \tau_{R}^{+}$ & $1 / 2$ & $+1 / 2$ & +1 & +1 \\
$e_{L}^{+}, \mu_{L}^{+}, \tau_{L}^{+}$ & 0 & 0 & +1 & +2 \\
\hline \hline Charged & EWK & Gauge & Bosons & \\
\hline \hline$W^{+}$ & 1 & +1 & +1 & 0 \\
$W^{-}$ & 1 & -1 & -1 & 0 \\
\hline \hline Neutral & EWK & Gauge & Bosons & \\
\hline \hline$Z^{0}$ & 1 & 0 & 0 & 0 \\
$\gamma$ & 0 & 0 & 0 & 0 \\
\hline
\end{tabular}

The experimental value of $\sin ^{2} \theta_{W}=0.2230 \pm 0.0004$ [19] [3] [5] [6] [7] [8].

\subsection{Structure Function}

The current experimental evidence currently suggests that leptons are point particles. On the other hand, the proton has been shown to have a substructure from mainly lepton-hadron experiments. For example, an electron (a leptonic point particle) is used to probe the substructure of a proton. The greater the momentum 
transfer of the probe and the target, denoted by $Q$, the more substructure of the proton can be observed. At momenta on the order of $1.0 \mathrm{GeV}$, protons appear to behave just like point particles. However, at tens of $\mathrm{GeV}$, protons look to be made up of valence quarks that determine the quantum numbers of a proton. At even higher momenta, protons contain gluons from gluon emission as well as quark-antiquark pairs from gluon splitting called "sea" quarks. In the end, all "valence" quarks, "sea" quarks, and gluons must conserve the quantum numbers when averaged over all momentum space. In fact, $55 \%$ of a protons momenta is carried by gluons and not by valence quarks. Aside from a fraction of the momentum of a proton being carried by quarks and gluons, the momentum transfer $Q^{2}$ of the two interaction partons also influences the amount of momentum that takes place in a given hard scattering process because as the $Q^{2}$ increases, more of the proton is seen by the probe. Both effects are combined to yield parton distribution functions which are indispensable in calculating hard-scattering cross-sections.

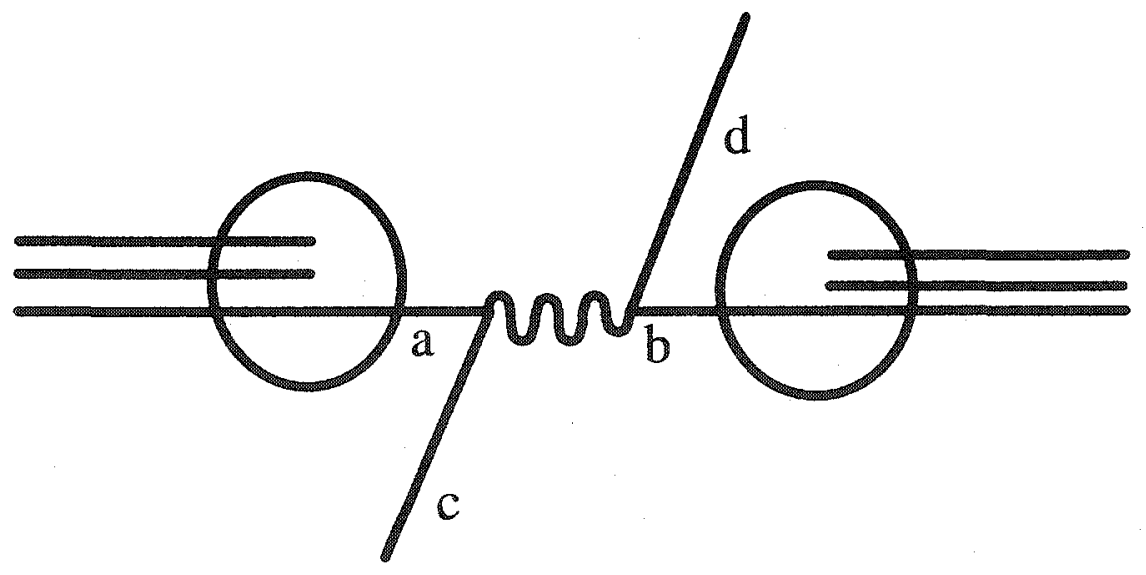

Figure 1.3: A hard scattering diagram. $a$ and $b$ are the incoming partons, and $c$ and $d$ are the outgoing partons. Other partons in the baryon (proton,antiproton) do not take part. The momentum transfer as well as the fraction of momentum of initial baryon that partons $a$ and $b$ have of the incoming proton and antiproton are used to determine the parton distribution function. 




Figure 1.4: The structure functions, which are given as the product of momentum fraction and the parton distribution function, that can be used to calculate the probability of finding a quark or gluon with a momentum fraction $x>0.1$. Notice how gluons dominate at small $\mathrm{x}[8]$.

Parton distribution functions are probability functions that quantify the amount of momentum a given parton of a proton carries, and parton distribution functions are important is the calculation of hard scattering $p \bar{p}$ cross-sections. If there are two interacting partons $a, b$ each carrying $x_{a}, x_{b}$ of the initial proton momentum, and the probability that a parton $a, b$ has fraction $x_{a}, x_{b}$ of the initial momentum of the proton and antiproton is given by [8]

$$
f_{a / p}\left(x_{b}, Q^{2}\right), f_{b, \bar{p}}\left(x_{b}, Q^{2}\right)
$$




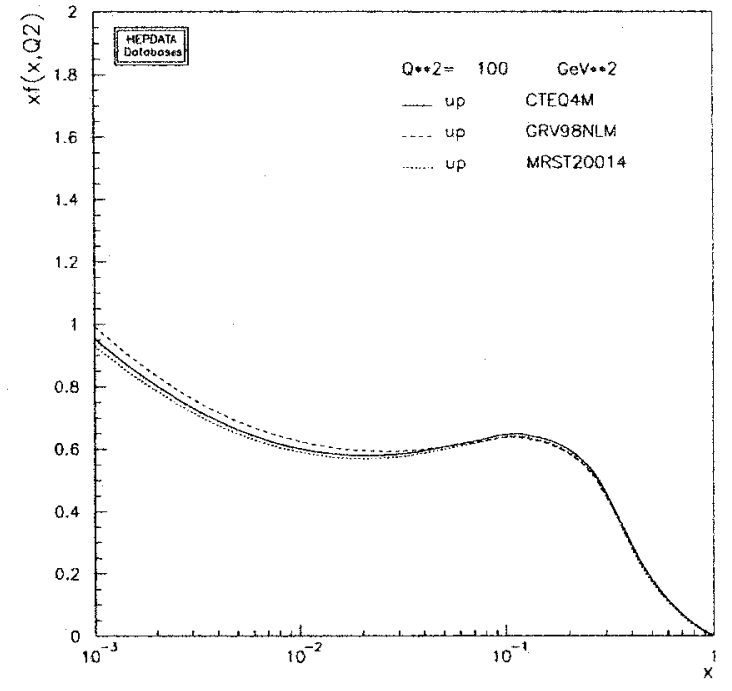

(a)

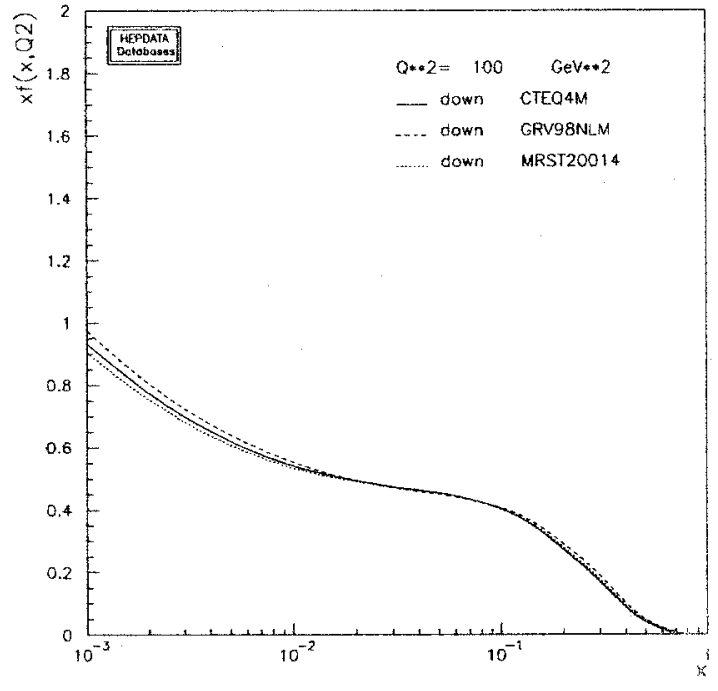

(b)

Figure 1.5: The momentum distributions for the u quark (left) and the $d$ quark (right) as predicted by a few global PDF models (CTEQ, MRS and GRV). At very low momentum fraction, these models diverge; however, all agree for $x>0.1$ [8].

In particular, the differential cross-section is calculated by multiplying the perturbative QCD cross-section, $\hat{\sigma}(a b \rightarrow c d)$, with both of these parton distribution functions $\left(f_{a / p}\left(x_{a}, Q^{2}\right), f_{b / \tilde{p}}\left(x_{b}, Q^{2}\right)\right)$ where $Q^{2}$ is the momentum transfer and $x_{a}, x_{b}$ is the fraction of the proton momentum carried by the given parton $a, b[8][20]$ :

$$
d^{2} \sigma(a b \rightarrow c d)=\hat{\sigma}(a b \rightarrow c d) f_{a / p}\left(x_{a}, Q^{2}\right) f_{b / \bar{p}}\left(x_{b}, Q^{2}\right) d x_{a} d x_{b}
$$

The parton distribution also should be consistent with the quantum numbers of the proton in the form of sum rules. For example, all quark-antiquark differences when averaged over all momentum fraction space must be equal to zero with the exception of $(u$ and $\bar{u})$ and ( $d$ and $\bar{d})$ quarks that equal 2 and 1 , respectively, because a proton has two valence up quarks and one valence down quark and all other sea 
quarks must average to 0 . The sum rules are given by [8] :

$$
\begin{gathered}
\int_{0}^{1}\left(f_{u / p}\left(x, Q^{2}\right)-f_{\bar{u} / p}\left(x, Q^{2}\right)\right) d x=2, \\
\int_{0}^{1}\left(f_{d / p}\left(x, Q^{2}\right)-f_{\bar{d} / p}\left(x, Q^{2}\right)\right) d x=1, \\
\int_{0}^{1}\left(f_{c, s, b, u, d, t / p}\left(x, Q^{2}\right)-f_{\bar{c}, \bar{s}, \bar{b}, \bar{u}, \bar{d}, \bar{t} / p}\left(x, Q^{2}\right)\right) d x=0 .
\end{gathered}
$$

In addition, the sum of all the individuals momentum of the partons must equal to momentum of the proton, and is given by [8],

$$
\sum_{a} \int_{0}^{1} d x x f_{a / p}\left(x, Q^{2}\right)=1
$$

where $a=g, u, d, s, c, b, t, \bar{u}, \bar{u}, \bar{d}, \bar{s}, \bar{c}, \bar{b}, \bar{t}$.

The data from a multitude of experiments, each covering various regions of $x$ and $Q^{2}$, is studied by various theoretical groups in order to model global PDF that are valid for most $x$ and $Q^{2}$. In figures 1.4 and 1.5, different models appear to converge at high momentum fraction values where the sea quark contributions are small [3] [5] [6] [7] [8].

\subsection{Fragmentation}

The mechanism of producing hadrons from quarks and gluons is one of the least understood phenomenon in high energy physics. After a $p \bar{p}$ interaction, outgoing high $p_{T}$ quarks and gluons cannot exist by themselves in isolation and must combine with other partons in order to form mesons and baryons. It is the consensus that fragmentation is independent upon the exact process ( $e^{+} e^{-}$collisions, $p \bar{p}$ collisions); 
instead, fragmentation depends only on the energy and type of the outgoing partons. The above assumption excludes possible color transfer issues which are dealt with in some models but may not be addressed in others. As of the effects of the type of outgoing partons, the gluons do fragment differently than the quarks. However, being difficult to distinguish between quark and gluon fragmentation, and in addition, not being the focus of this study, it will not be discussed further. So, the fragmentation theories derived from $e^{+} e^{-}$machines will also be assumed to be valid for $p \bar{p}$ interactions. In $e^{+} e^{-}$annihilation at center of mass energies $\sqrt{s}$, a hadron $h$ along with other particles $X$ are produced via intermediate vector bosons $\mathrm{V}=\gamma / Z^{0}$ in the following process $e^{+} e^{-} \rightarrow V \rightarrow h X$. Note that the process pbarp $\rightarrow V \rightarrow h X^{\prime}$ predictions is based mostly upon the data from the $e^{+} e^{--} \rightarrow V \rightarrow h X$ process.



Figure 1.6: A diagram for the fragmentation for the process $e^{+} e^{-} \rightarrow h X[21]$

The inclusive single-particle distribution of a hadron is described with a dimensionless quantity called the total fragmentation function for a hadron $h, F^{h}(x, s)$, 
in equation 1.11 [22]:

$$
F^{h}(x, s)=\frac{1}{\sigma_{\text {total }}} \frac{d \sigma}{d x}\left(e^{+} e^{-} \rightarrow V \rightarrow h X\right)
$$

where $s=$ square of center of mass energy of collision and $x=\frac{2 E_{h}}{E_{c m}}$. For a given hadron, the multiplicity of a hadron, $\left\langle n_{h}(s)\right\rangle$, is given by integration of fragmentation function over $x$, shown in equation 1.12 [22]:

$$
<n_{h}(s)>=\int_{0}^{1} d x F^{h}(x, s) .
$$

In particular, fragmentation functions are defined by equation 1.13 [22]:

$$
F^{h}(x, s)=\sum_{i} \int_{x}^{1} \frac{d z}{z} C_{i}\left(s ; z, \alpha_{s}\right) D_{i}^{h}\left(\frac{x}{z}, s\right)
$$

where the parton types are denoted by $i=u, \bar{u}, d, \bar{d}, \ldots$, and $g, C_{i}$ are coefficients for a process, $D_{i}^{h}$ is the universal fragmentation function, $z$ is the fraction of momentum of parton $i(x<f r a c x z<1)$, and $s$ and $x$ are defined as before. This is shown in Figure 1.6. The fragmentation simplifies dramatically if only lower order $\alpha_{S}$ terms are incorporated, with gluon emission being absent. With this approximation, $C_{g}=0$ for gluons and $C_{i}=g_{i}(s) \delta(1-z)$ for the rest of the quarks and antiquarks where $g_{i}(s)$ is proportional to the square of the charge of the parton $i$ where $i=u, \bar{u}, d$, $\bar{d}, \ldots$, and $g$. These approximations are not valid at Tevatron energies, and higher orders of $\alpha_{S}$ as well as gluon terms need to incorporated, and this will be elaborated later.

With the simplest approximations, a fragmentation function computed at a center of mass energy $\sqrt{s}$ is valid at all $\sqrt{s}$. However, QCD corrections introduce terms 
that give rise to so-called "scaling violations" in which fragmentation functions do indeed have an $\sqrt{s}$ dependence. These scaling violations may be predicted using the DGLAP (Dokshitzer-Gribov-Lipatov-Altarelli-Parisi) evolution equation given by equation $1.14[22][23]$ :

$$
t \frac{\partial}{\partial t} D_{i}(x, t)=\sum_{j} \int_{x}^{1} \frac{d z}{z} \frac{\alpha_{S}}{2 \pi} P_{j i}\left(z, \alpha_{S}\right) D_{j}\left(\frac{x}{z}, t\right)
$$

where $D_{j}$ is the fragmentation and $P_{j i}$ are splitting functions given by perturbative expansions shown in equation 1.15 [22]:

$$
P_{j i}\left(z, \alpha_{S}\right)=P_{j i}^{(0)}(z)+\frac{\alpha_{S}}{2 \pi} P_{j i}^{(1)}(z)+\ldots
$$

The splitting function, $P_{j i}$, is defined as the probability of getting parton $j$ from parton $i$ when parton $j$ has a fraction $z$ of the momentum of parton $i$. These equations work for all partons where $i=u, \bar{u}, d, \bar{d}, \ldots$, and $g$, and $j$ is summed over $u, \bar{u}, d, \bar{d}, \ldots$, and $g$. Once fragmentation functions are determined experimentally at center of mass energy $\sqrt{s}$, the fragmentation functions may be computed at another center of mass energy $\sqrt{t}$. As for the effect of the scaling violation, the $x$ distribution is moved towards lower values as the scale increases.

Fragmentation functions are similar to structure functions. Structure functions represent the probability distribution of a parton in a hadron carrying a certain fraction of the momenta of the hadron. Likewise, fragmentation functions are the probability distributions that show the odds that a parton with a given momentum will make up a hadron with another given total momentum. Both fragmentation and structure functions are incalculable using perturbation theory. Furthermore, the simplest model would result in scale-independent $x$ distributions with scaling 
violations being derived once the QCD corrections are included. Moreover, distributions determined at a particular center of mass energy $\sqrt{s}$ may be determined for $\sqrt{t}$ using evolution equations.

At low $Q^{2}$, perturbation theory breaks down, and the running coupling constant grows. It turns out that terms which contribute to the fragmentation function that do not have log can be ignored [24]. Furthermore, for gluons, only terms on the order of $\alpha_{S} l n^{2} Q^{2}$ (note: $Q^{2}$ is actually divided by a constant to make argument in the logarithm dimensionless, and this constant depends upon the details of the calculation) and above may be kept (Double Logarithmic Approximation [25]). Significant simplification of higher orders may be made by applying angular ordering. Angular ordering shows that gluons emitted successively from a parent parton are approximately equivalent to a single diagram with angular ordered emissions. In other words, partons are do not radiate at angles at greater angles than those of the prior emission. Thus, emission terms that are collinear may be summed, and the resulting calculation includes less terms than one which does not use this approximation. The Double Logarithmic Approximation with angular ordering plus some additional single log contributions comprise the Modified Leading Log Approximation (MLLA) [26] [27]. The MLLA is implemented in many Monte Carlo programs and agree quite well with the data. The DGLAP equation with the MLLA are denoted by equation 1.16 [21]:

$$
t \frac{\partial}{\partial t} D_{i}(x, t)=\sum_{j} \int_{x}^{1} \frac{d z}{z} \frac{\alpha_{S}}{2 \pi} P_{j i}\left(z, \alpha_{S}\right) D_{j}\left(\frac{x}{z}, z^{2} t\right)
$$

[21] [22] [28]. 


\subsection{Hadronization Models}

Fragmentation does not speculate as to the underlying mechanism of how partons combine into hadrons; it only derives the inclusive hadron spectra and how these spectra scale with center of mass energy. Only non-perturbative and phenomenological hadronization models attempt to explain how partons merge into hadrons. Although there at least a couple major of hadronization models, when combined with fragmentation, reproduce the data quite well, only the cluster and string models will be described.

In Figure 1.7, a pictorial overview of the hadronization process will now be described. First, there is a hard scattering process that produces outgoing partons. The significant parameter which governs the energy and type of outgoing partons is the hard scattering scale $M_{J J}$ where $M_{J J}$ is the dijet mass. Then, an outgoing parton will shower into less energetic partons until the daughters would have transverse momenta below $Q_{e f f}$. Parton showering is a perturbative process where the uncertainty increases as the value of $Q_{e f f}$ decreases. Below this threshold of $Q_{e f f}$, the parton showering will cease, and groups of partons will combine into hadrons using one of the phenomenological hadronization models. There are two major ones, the string and cluster models; they will both discussed shortly. The last scale, order of $\frac{1}{m_{\pi}}$, is where hadronization terminates.

The value at which perturbative fragmentation ends and non-perturbative hadronization begins occurs, as stated above, at some transverse momenta scale factor called $Q_{\text {eff }}$. Since perturbative theories are quantitatively and qualitatively better understood, a lower value for $Q_{e f f}$ is more desirable because more of the calculation is based upon perturbation theories rather than phenomenological ones. However, if $Q_{\text {eff }}$ is too low and is on the order of $(200-400 \mathrm{MeV})$, the perturbative theory 


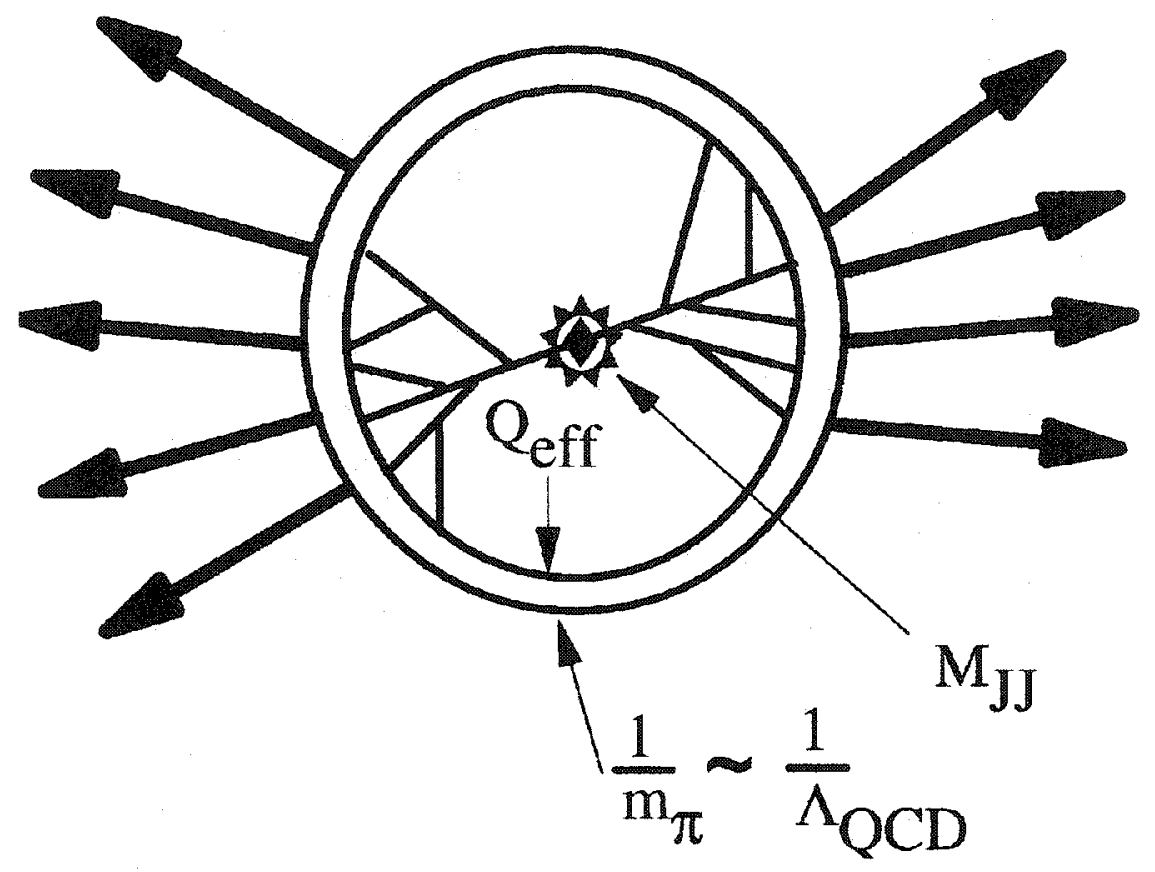

Figure 1.7: A pictorial description of jet fragmentation with the three scales $M_{J J}$, $Q_{e f f}$, and $\frac{1}{m_{\pi}}$. The hard-scattering scale $M_{J J}$ appears at the center. $Q_{\text {eff }}$ is the scale at which the parton showering ends and the hadronization begins. The last scale, order of $\frac{1}{m_{\pi}}$, is where hadronization terminates [28].

begins to break down because the corrective terms become too large. One big question is how to implement the predictive parton calculations to the data through a not-well-understood non-perturbative hadronization process? Any given outgoing parton above a certain $Q_{e f f}$ will shower into other partons, then the partons at or below $Q_{e f f}$ will combine into mesons and baryons in another process called hadronization. The value $Q_{\text {eff }}$ can only be determined experimentally. In one study, $Q_{\text {eff }}=256 \pm 13 \mathrm{MeV}[28]$.

Hadronization models are based upon the Local Parton-Hadron Duality (LPHD) Hypothesis [29] [30]. The LPHD hypothesis assumes that hadronization occurs after parton showering. In addition, the hadrons and the partons they are derived from are closely correlated. Moreover, color effects from the two outgoing partons which 
generate other hadrons are assumed to be uncorrelated. This is believed to be the case because by the time the partons fragment, they are far apart from each other. Consequently, although hadron and parton distributions differ, the inclusive average of these distributions are the same. In particular, the multiplicity and momenta distributions of hadrons and partons differ only by a normalization constant. The more particles produced (or the higher energy of the fragmenting quark or gluon), the more accurate the LPHD assumption [21] [28].

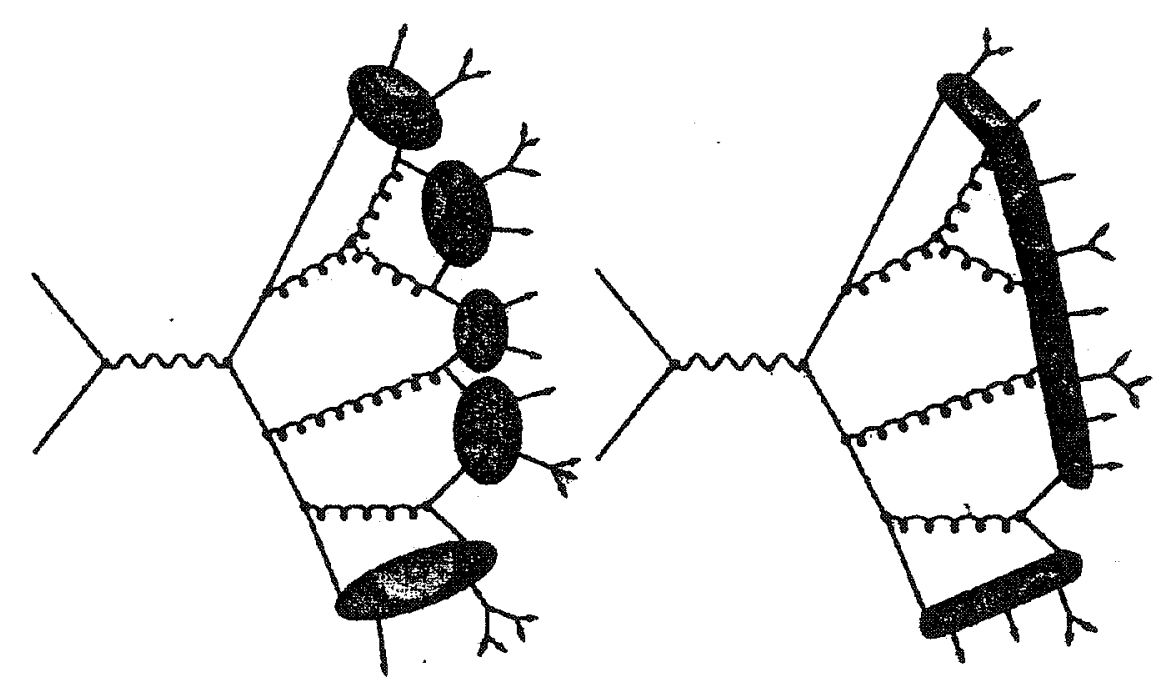

Figure 1.8: The pictorial diagrams of the cluster (left) and the string (right) hadronization models [21].

At some transverse momenta, quarks and gluons are converted to hadrons in a process called "hadronization". There are many different hadronization models that adopt the LPHD hypothesis. The cluster model will be later compared with the data in this analysis, and HERWIG (the Monte Carlo generator used in this study) adopts this hadronization model. In this method, once the parton showering process terminates, all gluons are converted into quark antiquark pairs. Each group of quarks and antiquarks in a particular region of phase space are clumped into 
clusters, and depending on the mass of the cluster, can either decay directly into hadrons or divide itself into smaller clusters and decay directly into hadrons later. Unstable hadrons decay according to decay modes and branching ratios until masses of the lightest hadrons are attained. All this procedure considers allowable phase space so that the momenta and energy of all the final state hadrons is equal to the momenta and energy of the initial cluster of quarks and antiquarks. The exact ratios of lighter quarks to heavier quarks are close to that of another model, the string model (see next paragraph). The cluster model also tends not to reproduce baryon and heavy quark production well [21] [22] [28] [31] [32].

Although not compared in this analysis, the string model is another popular hadronization model worth mentioning. The many people who use the Pythia Monte Carlo generator use the string model. In this model, the color fields between partons fragments rather than the partons. In particular, as a quark-antiquark pair separates, the linear color field between them increases until there is enough energy stored up to produce two quark-antiquark pairs. The color field is represented as a string believed to have a mass density $\kappa$ of approximately $1 \mathrm{GeV} / \mathrm{fm}$. The new quark-antiquark pairs are determined via a tunneling probability function $\exp \left(-\pi m_{q, \perp}^{2} / \kappa\right)$ where the transverse mass is given by $m_{q, \perp}^{2}=m_{q}^{2}+p_{q, \perp}^{2}$. It is this tunneling probability that suppresses the production of heavy quarks; u:d:s:c is generated with approximately the following ratios of 1:1:0.3:10-11. This is an iterative process that terminates once quark-antiquark pairs are no longer above the mass of hadrons. Gluon emission removes energy from the color field and appear as kinks in the string. As for baryon production, a diquark pair may also be generated via tunneling and combine with single quark to form a baryon. The string model is an improvement over the cluster model when it comes to baryon produc- 
tion [21] [22] [33] [34]. Both hadronization models are shown in Figure 1.8 [21].

In the end, there are groups of particles, resulting from parton showering and hadronization process, traveling in the general direction of an initial hard-scattering parton. These particles will be considered as candidates for an entity called a "jet". Jets will be discussed at great length in Chapter 6. Of great interest in this analysis, the strangeness content contained with these jets.

\subsection{Motivation of the Analysis}

The motivation of this study is to test the treatment of the production of strangeness in the fragmentation and the hadronization models supported by earlier experiments. Most strangeness production theories are derived from $e^{+} e^{--}$machines such as LEP, and there is the question of whether these models are also valid for $p \bar{p}$ collisions of the Tevatron. In particular, there are two competing hadronization models: the cluster model and the string model. The cluster model and the string model are incorporated in HERWIG and Pythia generators, respectively. Although not discussed further, the string model is not expected to vary much from the HERWIG Monte Carlo cluster model. In order to test the cluster model, certain physics quantities will be computed in the data and contrasted with those predicted by the HERWIG Monte Carlo based upon the cluster model. In addition, other physics values will be compared directly to those from $e^{+} e^{-}$experiments. This is the first time anyone has studied strangeness in jets in $p \bar{p}$ collisions.

The object mostly closely tied with that of a fragmenting quark or gluon is a cluster of particles going in the same direction as the initial quark or gluon, and this quantity is called a "jet". Typically, jets consists of charged and neutral particles within a region of space and whose identification depends mostly upon 
energy deposition in the calorimeter. By computing the number of particles having a strange quark inside jets, the data may be measured against those from the HERWIG Monte Carlo.

In this analysis, the production of $K_{S}$ in jets will be taken as the barometer of strangeness production since $K_{S}$ are abundant and contain a strange quark. Thus, the number of $K_{S}$ per jet, $\frac{N_{K S}}{j e t}$, will be one of the key measurements. In one mode, a $K_{S}$ conveniently decays into two charged pions. Although the $K_{S}$ may not be traced using the tracking chamber because it is neutral, the parent mass of $K_{S}$ may be reconstructed from its two charged pions daughters. In this manner, the production of $K_{S}$ may be calculated.

Also, the number of charged particles within a jet, $\frac{N_{\text {track }}}{j e t}$ quantities can be compared to the cluster model. This value is a check on the overall particle generation of the cluster model. The same can be said about the momenta spectra of the $K_{S}$ and tracks inside jets and their production ratio to each other, the number of $K_{S}$ divided by the number of charged tracks, $\frac{N_{K S}}{N_{\text {track }}}$. It is important not only examine strangeness production but also its production relative to those of charged particles. Others have studied track production in dijets and track production of jets defined strictly with tracks, but nobody at the CDF has examined track production in jets determined via calorimetry as a stand-alone entity.

One great test of any model is its ability to accurately predict the data in regions that were previously inaccessible. With $e^{+} e^{-}$machines, only strangeness production in jets below $50 \mathrm{GeV}$ has been published and the upper limit of jet $E_{T}$ for future LEP publications may confidently be placed at $100 \mathrm{GeV}$. On the other hand, the Tevatron collisions are at much higher center of mass energies, and consequently, strangeness production will be studied in jets up $150 \mathrm{GeV}$ in this analysis. The 
strangeness content in jets may be extended $30 \%$ higher over all other experimental data.

If the cluster model is accurate, those values calculated from the HERWIG Monte Carlo will be similar to the data. A discrepancy between HERWIG and the data would imply that the cluster model would need to adjust the strangeness content of fragmenting quarks and gluons. Furthermore, disagreements may arise from an increase in jet $E_{T}$ or from an overall difference in particle production. In any case, currently for the string model (and cluster model is comparable), the production ratios of the up, down, and strange quarks are approximately set at 1:1:0.3 for all jet $E_{T}$.

Moreover, through fragmentation plots, these results may be directly measured against other experiments. Being just the $p_{T}$ of the $K_{S}$ divided by the jet $E_{T}$, the fragmentation is a measure of overall $K_{S}$ production. The fragmentation function is believed to be independent of initial energy of the partons. These $p \bar{p}$ Tevatron results may be contrasted with those from $e^{+} e^{-}$collisions. If the shape of the spectra are similar and there is an offset (the Tevatron plot lower than the others), this may be evidence of a jet $E_{T}$ dependence (scaling violation).

Prior to any jet analysis, $K_{S}$ production in the Minimum Bias set is studied and compared to other analyses in order to support the $K_{S}$ production in jets analysis. For example, the $\mathrm{c} \tau$ measurement of $K_{S}$ will test the reliability of the efficiencies. Other quantities such as invariant cross-section are contrasted to other publications among quantities.

There are many questions: Is the cluster model correct? Is $K_{S}$ production too high or too low in jets? Are these ratios of up:down:strange quarks correct? If so, are particles generated at the appropriate $p_{T}$ spectra? If $K_{S}$ production is too low, 
is that because the cluster model generates too few $K_{S}$ or too few particles overall? If these ratios are correct, are do they hold for all measured jet $E_{T}$ ? How do the Tevatron's $p \bar{p}$ and the $e^{+} e^{-}$collisions from other experiments compare? Is there evidence of scaling? Are these results consistent with those of predecessors?

Much discussion and work to be done. Let us begin with the tools in this analysis, the Tevatron and the CDF detector. 


\section{Chapter 2}

\section{The Experiment}

The Fermi National Laboratory is one of the world's most renowned science research facilities. Located 35 miles west of Chicago on 7000 acres of land, over 2200 scientists from 36 states and 20 countries push both science and technology to new limits. From particle physics to astrophysics, from detector design to accelerator innovations, and from the development of theoretical models to the discovery of the top and bottom quarks, Fermilab has been and will continue to be an excellent place for first-class research. From 1991-1996, trillions of $p \bar{p}$ collisions at center of mass energies of $1.8 \mathrm{TeV}$ took place using the $6.28 \mathrm{~km}$ circumference Tevatron accelerator buried $30 \mathrm{ft}$ underground. Since then, the Tevatron and its detectors, CDF and D0, have undergone complex upgrades. In particular, by replacing the Main Ring with the Main Injector, the ability of recycling protons was achieved. This new feature should not only increase the center of mass collision energy to $1.96 \mathrm{TeV}$ but also increase the number of collisions by an order of magnitude. Detector improvements were mainly aimed at advancing resolution, acceptance, and readout. Although the Run II detector is extremely interesting, this analysis is based on Run I data (19911996 data). Hence, only the manner in which $p \bar{p}$ interact at $1.8 \mathrm{TeV}$, the Run I CDF detector, and the data acquisition system will be briefly described [6] [7] [8] [35]. 


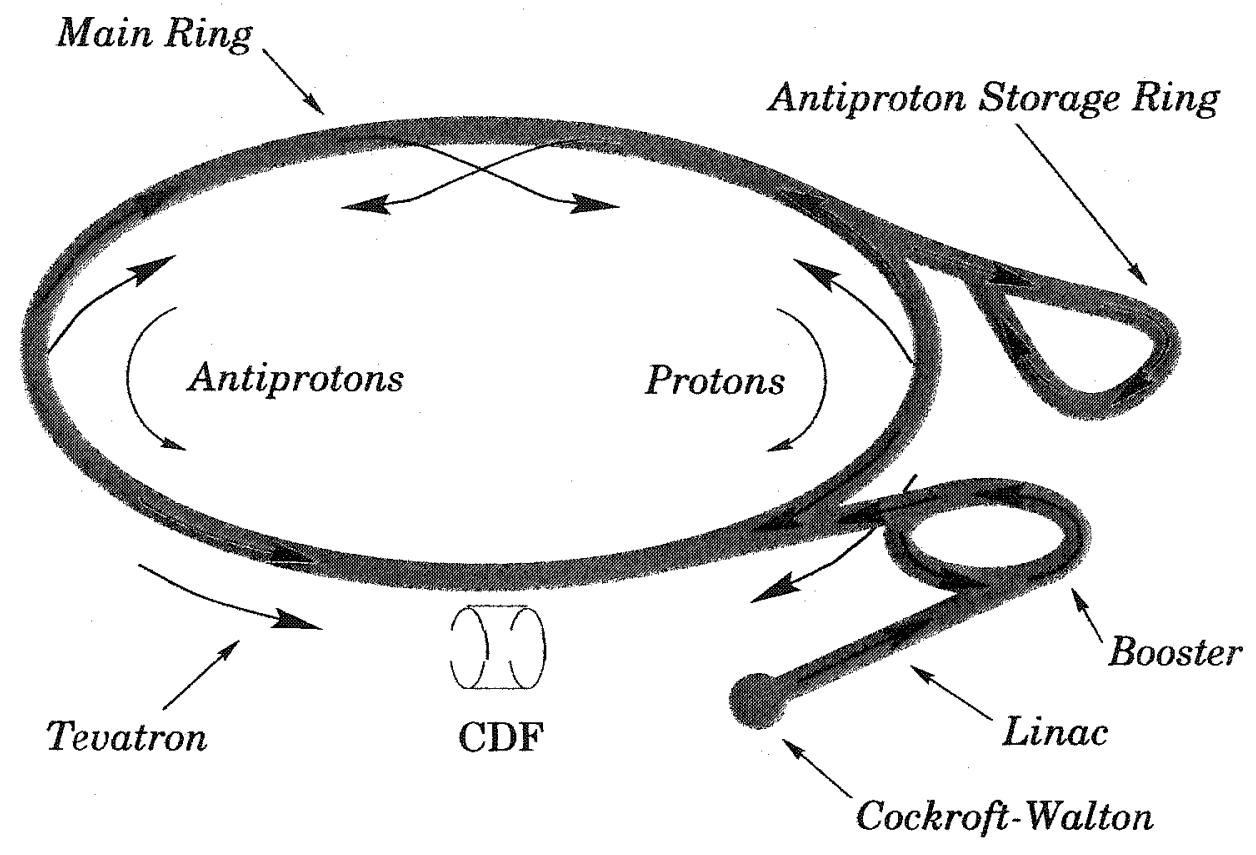

Figure 2.1: A schematic of the Tevatron accelerator at the Fermi National Accelerator Laboratory. The Cockcroft-Walton, Linac, Booster, Main Ring, and the Tevatron are used to collide protons and antiprotons at center of mass energies of $1.8 \mathrm{TeV}$. Note that CDF is located at one of the collision points.

\subsection{The Fermilab Tevatron}

To have $p \bar{p}$ collisions at center of mass energies of $1.8 \mathrm{TeV}$, protons and antiprotons are accelerated to $900 \mathrm{GeV}$ using a five stage accelerator process as well as use sophisticated techniques to accumulate and store antiprotons. The first stage of the process consists of a Cockcroft-Walton accelerator taking negative Hydrogen to energies of $750 \mathrm{keV}$, and then a linear accelerator (LINAC) ramps up the energy of these ions to $400 \mathrm{MeV}$. Electrons from the $H^{-}$ions are removed leaving only the protons to be injected into the Booster. The Booster brings protons up to $8.0 \mathrm{GeV}$ using its magnetic dipoles to force the particles to follow circular orbits at almost constant orbital radius and constant orbital frequency by increasing the dipole fields with the momentum of the particles. From the Booster, protons are brought up to 


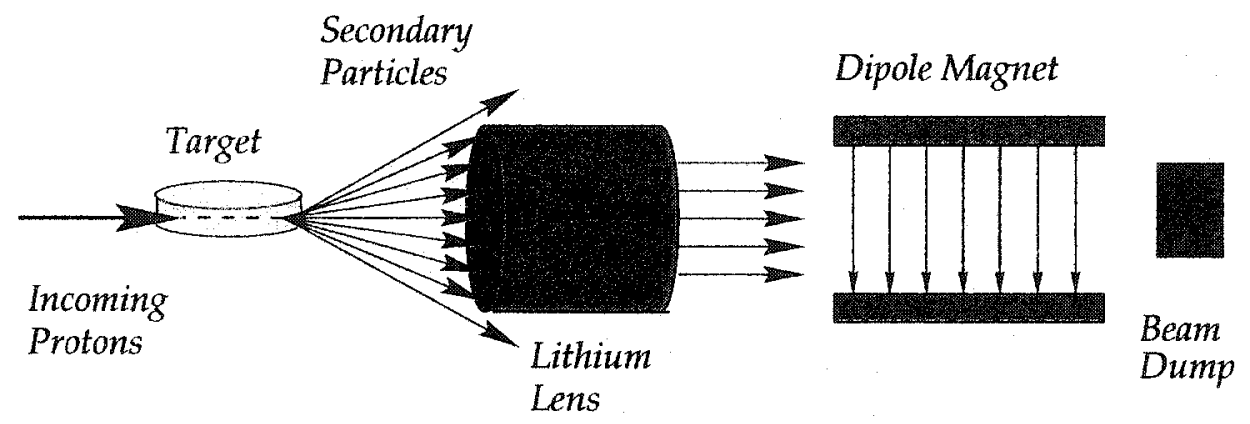

Figure 2.2: In order to produce antiprotons, protons collide into a Nickel target to generate secondary particles from which antiprotons are filtered and focused into a beam using a cylindrical Lithium lens.

$150 \mathrm{GeV}$ with 774 dipole magnets and 240 quadrupole magnets in the Main Ring. The dipole magnets are for steering, as stated earlier, and the quadrupole magnets are for focusing the beam.

By directing $120 \mathrm{GeV}$ proton into a Nickel target to create many secondary particles, antiprotons are filtered out and focused into a beam using a cylindrical Lithium lens. This process generates $10^{7}$ antiprotons with every collision with the fixed target. The antiprotons are stored in the Debuncher at energies of $8 \mathrm{GeV}$ where the momentum spread is reduced, and the antiprotons are then stockpiled in the accumulator until approximately $10^{10}$ antiprotons are collected. From here, the antiprotons are placed into the Main Ring rotating counter to the protons until both are at energies of $150 \mathrm{GeV}$. The last acceleration stage is when both protons and antiprotons are accelerated to energies of $900 \mathrm{GeV}$ inside the Tevatron, with protons orbiting counterclockwise and antiprotons orbiting clockwise, until center of mass collisions of $1.8 \mathrm{TeV}$ can be attained. The Tevatron is concentric with the Main Ring, but it incorporates 774 superconducting dipole magnets and 216 superconducting quadrupole magnets.

6 bunches of protons and 6 bunches of antiproton collide every $3.5 \mu s$ at up 
to 4 interaction points. However, there are only 2 collider detectors, CDF and D0, so electrostatic separators are used to prevent bunch crossings at the other collision points. A collision can occur only when a proton and an antiproton bunch overlap. Each bunch has an average radius of $40(25) \mu m$ for Run $1 \mathrm{~A}(\mathrm{~B})$ and a Gaussian distribution with a $\sigma$ of $30 \mathrm{~cm}$ in $z$. Also, there are $2.0 \times 10^{11}$ protons in the a proton bunch, and normally there are about 10 times less antiprotons in an antiproton bunch.

The number of collisions can be calculated using

$$
R=\mathcal{L} \sigma_{\text {int }}
$$

where $R$ is the rate of events, $\sigma_{i n t}$ is the cross-section of a given interaction, and $\mathcal{L}$ is a quantity called the luminosity. The luminosity is approximately given by

$$
\mathcal{L}=\frac{f n_{p} n_{\bar{p}}}{4 \pi \sigma_{p} \sigma_{\bar{p}}}
$$

where $n_{p}$ and $n_{\bar{p}}$ are the number of particles in each bunch, $f$ is the frequency of collisions, and $\sigma_{p}$ and $\sigma_{\bar{p}}$ are the Gaussian transverse beam profiles. In general, nature fixes the cross-section of a given process, and increasing luminosity is very important in order to increase the data used for analyses. Reducing the beam profiles, increasing the collision rate, and increasing the number of particles in each bunch are ways to achieve this goal. For example, with a given $p \bar{p} \sigma_{i n t}$ of $50 \mathrm{mb}$ at 1.8 $\mathrm{TeV}$, and a Run $1 \mathrm{~B}$ average $\mathcal{L}$ of $1.6 \times 10^{31} \mathrm{~cm}^{-2} \mathrm{~s}^{-1}$, the collision rate is 800 thousand interactions per second. Since only a few events can be written onto $8 \mathrm{~mm}$ tape per second, triggers are used to select events of interest as much as the acceptance rate will permit. Luminosity decreases with time because protons and antiprotons 


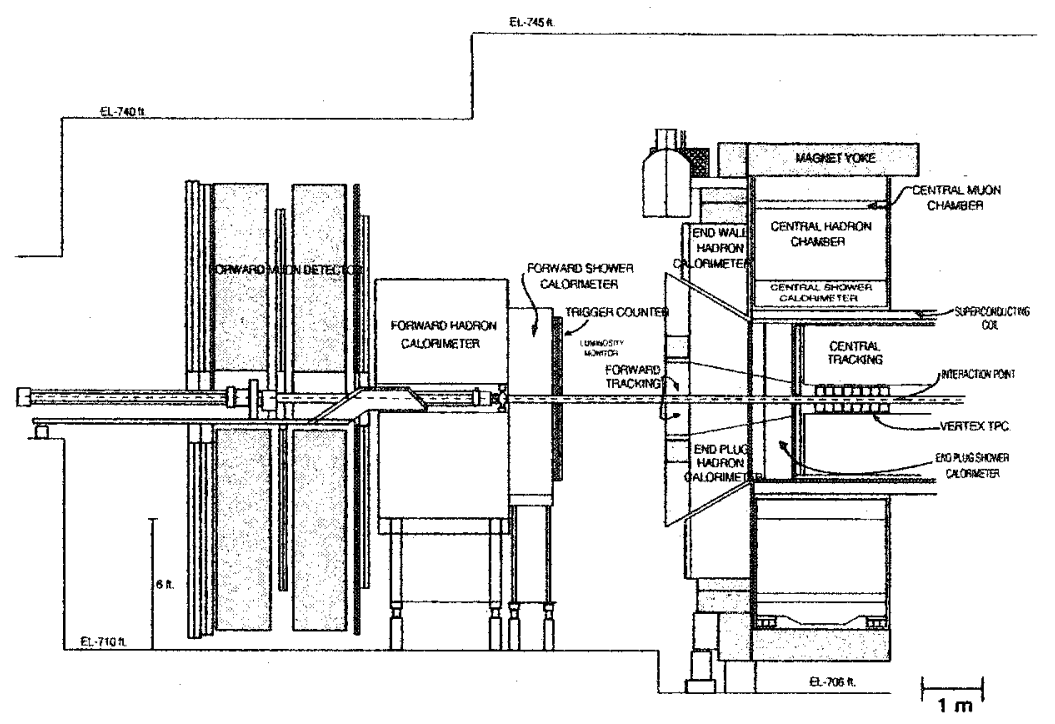

Figure 2.3: The forward half of the CDF detector. The CDF detector is azimuthally and forward-backward symmetric about the nominal interaction point.

are lost either to beam gas collisions or $p \bar{p}$ collisions as well as the beam increases its beam profiles $\sigma_{p}$ and $\sigma_{\bar{p}}$. Hence, each Tevatron store of protons and antiprotons lasts about 20 hours before replacing the bunches becomes necessary [6] [7] [8] [35].

\subsection{Collider Detector at Fermilab (CDF)}

The Collider Detector at Fermilab (CDF) is centered around one of six nominal interaction points around the Tevatron, and CDF collects a wide range of data from $1.8 \mathrm{TeV} p \bar{p}$ collisions. Using the charge, position, momentum, and energy deposition information obtained from the $27 \mathrm{~m}$ long, $10 \mathrm{~m}$ high, and over $2300 \mathrm{~kg}$ detector, a wide variety of events can be analyzed. In the rest of the chapter, CDF will be briefly discussed with special emphasis on the components which are relevant to this analysis. 


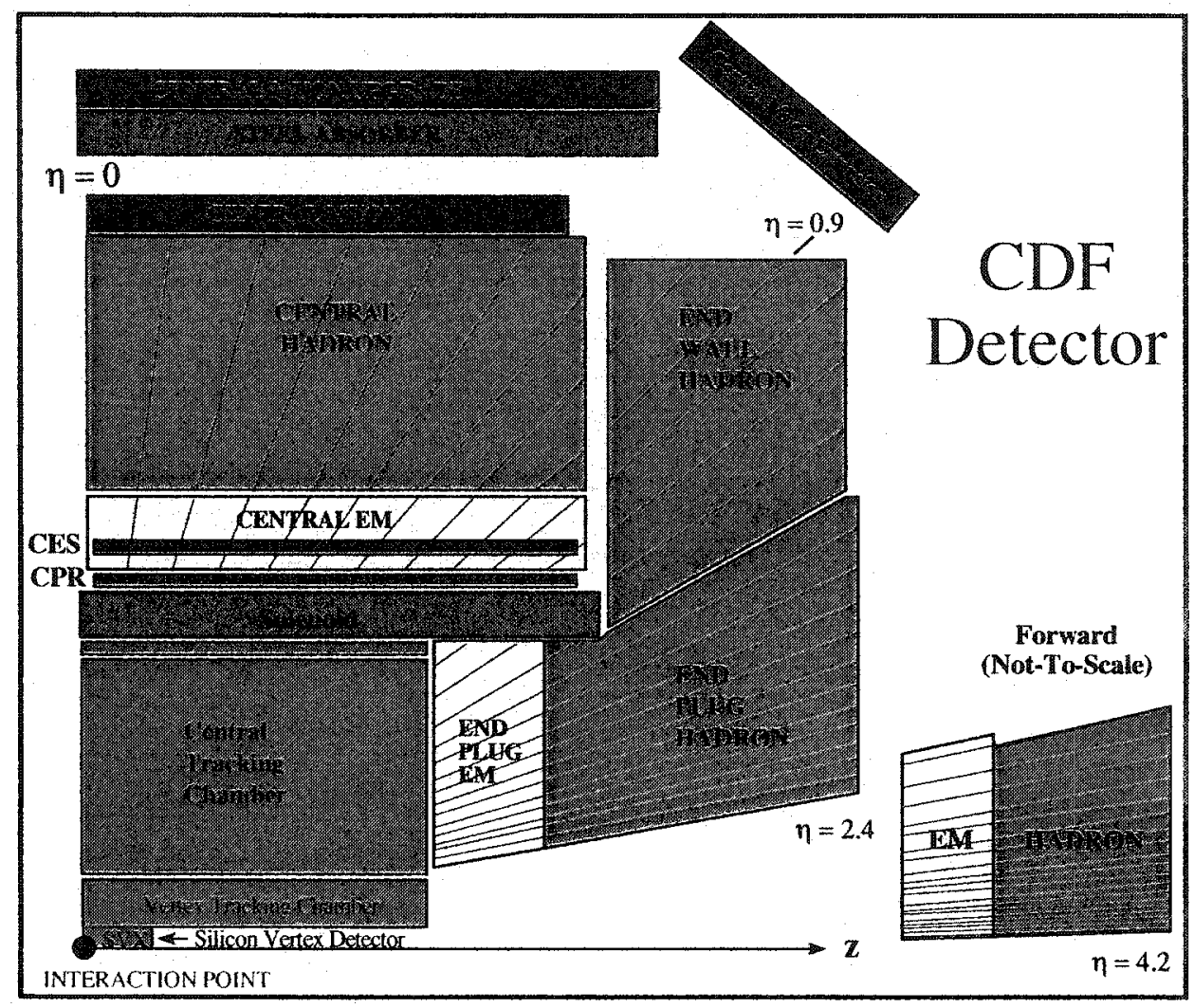

Figure 2.4: A quadrant of the CDF detector.

Since CDF is forward-backward and azimuthally symmetric, cylindrical coordinates are employed. CDF uses a right-handed coordinate system where the positive $z$-axis is the direction of the beam of protons, the $y$-axis is directly upward, and the $\mathrm{x}$-axis is radially outwards. The polar angle $\theta$ is measured with respect to the $z$-axis, and the azimuthal angle $\phi$ is the angle viewed from the $+z$-axis is measured counter-clockwise.

One particularly useful quantity called rapidity is defined as

$$
y=\frac{1}{2} \ln \left(\frac{E+P_{z}}{E-P_{z}}\right)
$$

Under Lorentz boosts with a velocity $\beta$ along the $z$-axis in reference to an inertial 




Figure 2.5: The diagram of a single SVX barrel.

frame, the transformation of rapidity is $y \rightarrow y+\tanh ^{-1} \beta$ and the difference between two rapidity quantities is Lorentz invariant. For highly relativistic particles, $p \gg m c$, rapidity can be simplified to

$$
\eta=-\ln \left(\tan \frac{\theta}{2}\right) .
$$

This quantity is called pseudorapidity and is approximately equal to rapidity. There are actually two useful forms of pseudorapidity. The first is called detector pseudorapidity $\left(\eta_{\text {detector }}\right)$, and it is calculated using the nominal interaction point as the origin (geometric center of the detector). The latter is called event pseudorapidity $(\eta)$ and this quantity is measured with respect to the interaction vertex of $p \bar{p}$ collisions. The distribution of the interaction vertex in z closely resembles a Gaussian 


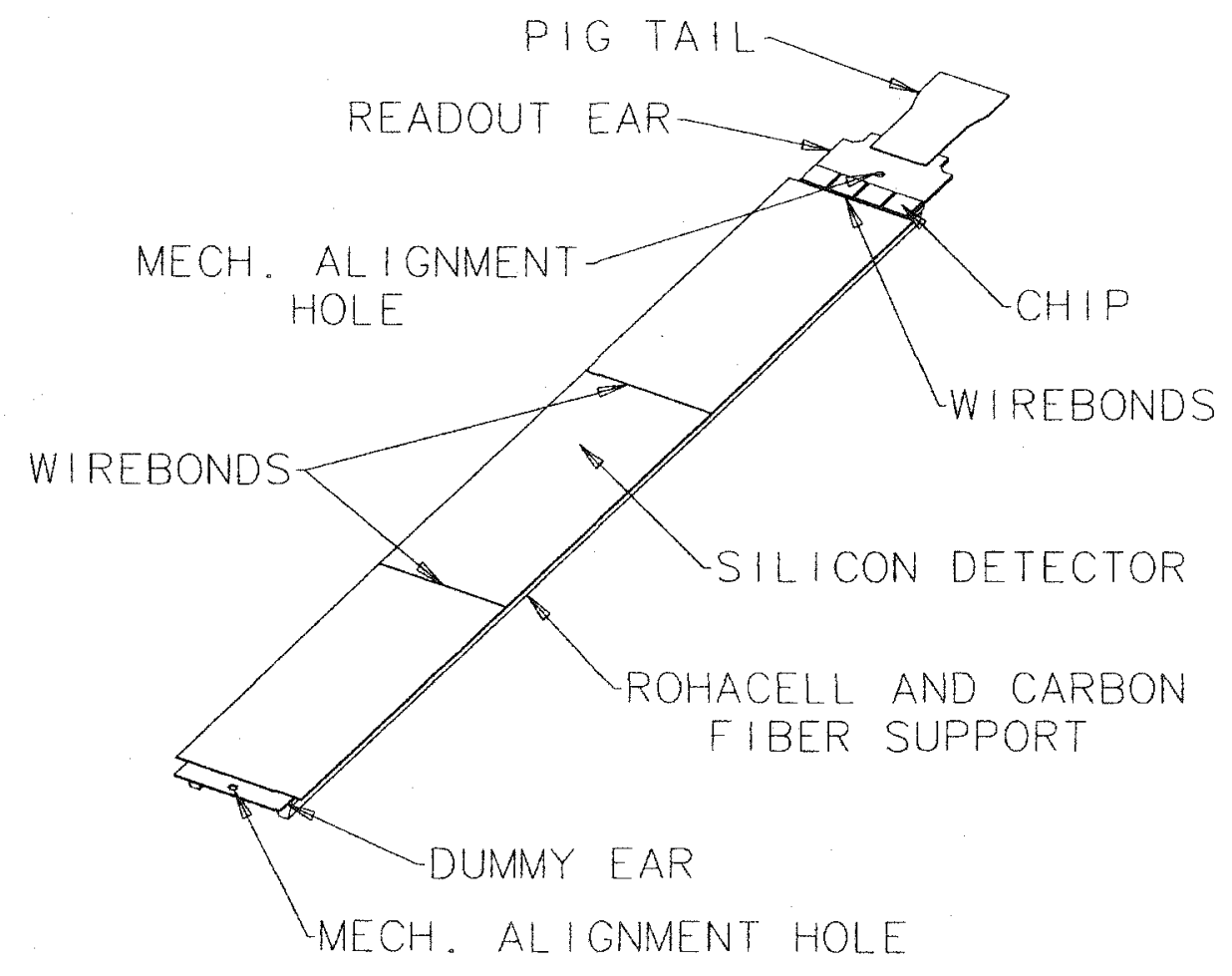

Figure 2.6: A figure of a SVX ladder.

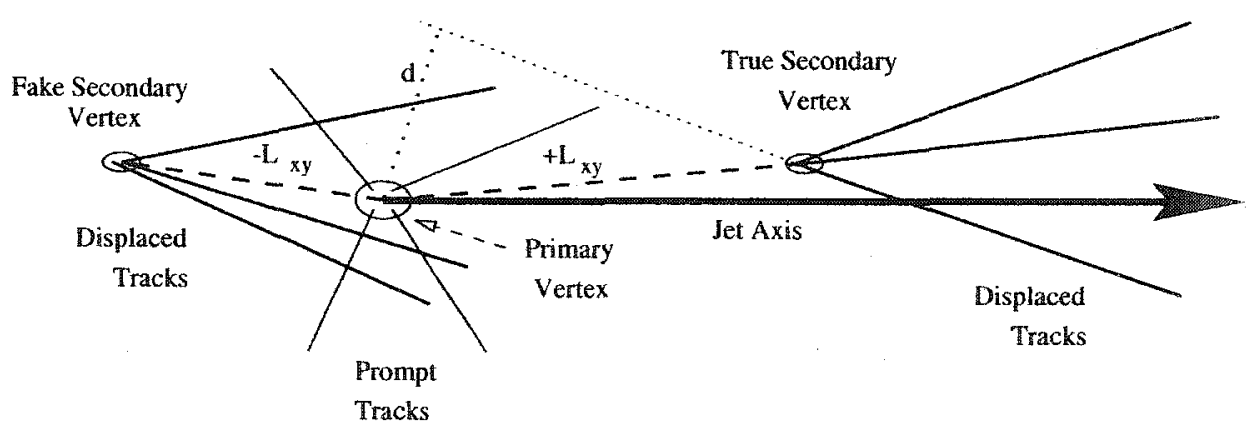

Figure 2.7: A picture of tracks intersecting at a secondary vertex which is displaced $L_{x y}$ from the primary vertex.

with $\sigma$ of $30 \mathrm{~cm} \mathrm{[6]} \mathrm{[7]} \mathrm{[8]} \mathrm{[35].}$ 


\subsubsection{Central Tracking Systems}

The Central Tracking systems are designed to determine the trajectories of charged particles for the purpose of momentum and charge measurements. In addition, electromagnetic clusters without tracks can be used for photon identification. The Silicon Vertex Detector (SVX) is positioned closest to the beamline. The SVX is a series of silicon microvertex detectors which yield high precision measurements near the interaction region. Beyond the SVX, the Vertex Tracking Chamber (VTX) gives $r-z$ information of the tracks. The Central Tracking Chamber, which surrounds both the SVX and VTX, is instrumental in 3D track reconstruction. The latter two detectors are wire drift chambers. Enclosing all of the Central Tracking systems is a $4.8 \mathrm{~m}$ long $\mathrm{NbTi} / \mathrm{Cu}$ superconducting solenoid with a radius of $1.5 \mathrm{~m}$ that generates the 1.41 Tesla axial magnetic field. This field causes the charged particles to follow the helical trajectories from which momenta and charge can be extracted.

\section{Silicon Vertex Systems (SVX)}

The Silicon Vertex Detector (SVX) primary purpose is to precisely determine tracking in $r-\phi$ in order to identify tracks intersecting away from the primary vertex, called "secondary vertices", that are useful in locating B-hadrons with $\mathrm{c} \tau$ on order of $300-400 \mu \mathrm{m}$. It is important to note that although this analysis depends heavily on secondary vertices for $K_{S}$ identification, the SVX is not used in this analysis. SVX can indeed be used to find $K_{S}$ and studies have been done at CDF. However; the $\mathrm{c} \tau$ of the $K_{S}$ are on the order of $2.6786 \pm 0.0024 \mathrm{~cm}[9]$ so these particles have long enough $\mathrm{c} \tau$ for the resolution of the CTC to be more than sufficient.

The SVX consists of a pair of $25.5 \mathrm{~cm}$ long barrels that have a gap of $2.15 \mathrm{~cm}$ between them. Each barrel is divided azimuthally into 12 wedges, and each wedge has 4 layers of single-sided silicon called ladders. Every ladder has 3 silicon wafers, 
Table 2.1: Summary of the SVX characteristics. Given below is the crystal width, active area, the number of readout strips, and the number of readout chips for a given layer of SVX.

\begin{tabular}{|c|c|c|c|c|}
\hline Layer & $\begin{array}{c}\text { Crystal } \\
\text { Width (cm) }\end{array}$ & $\begin{array}{c}\text { Active Area } \\
\text { Width (cm) }\end{array}$ & $\begin{array}{c}\text { Readout } \\
\text { Strips }\end{array}$ & $\begin{array}{c}\text { Readout } \\
\text { Chips }\end{array}$ \\
\hline 0 & 1.6040 & 1.5360 & 256 & 2 \\
1 & 2.3720 & 2.3040 & 384 & 3 \\
2 & 3.1400 & 3.0720 & 512 & 4 \\
3 & 4.2930 & 4.2240 & 768 & 6 \\
\hline
\end{tabular}

which are $300 \mu \mathrm{m}$ thick and $8.5 \mathrm{~cm}$ long, and is rotated by $3^{\circ}$ about its length to provide overlap. In addition, each ladder increases in width, the number of readout strips, and the number of readout chips as the position of the ladder increases radially. Each wafer has a strip pitch of $60 \mu \mathrm{m}$ for the first 3 layers closest to the beampipe and $55 \mu \mathrm{m}$ for the outer most layer, and every wafer is surrounded with conducting strips. All silicon strips are parallel to the $z$-axis, and hence can only obtain $r-\phi$ information of the tracks. The SVX covers $60 \%$ of the $p \bar{p}$ interactions with its detector pseudorapidity coverage of $\left|\eta_{\text {detector }}\right|<1.9$.

Once the silicon is biased with a potential and an ionizing particle goes through the silicon, a large quantity of electron-hole pairs are generated. The electrons which are excited to the conduction band are attracted to the large potential difference of the conducting strips. The voltage drop of the strip is proportional to that of the ionization, and the strips are readout with electronics. In total, there are 46,080 strips channels in the SVX that result in a resolution of about $10 \mu \mathrm{m}[6][7][8][35]$.

\section{Vertex Detector (VTX)}

The VTX detector is a drift-time proportional chamber used to more accurately describe where, in the $30 \mathrm{~cm}$ Gaussian of $p \bar{p}$ collisions, an event actually took place 

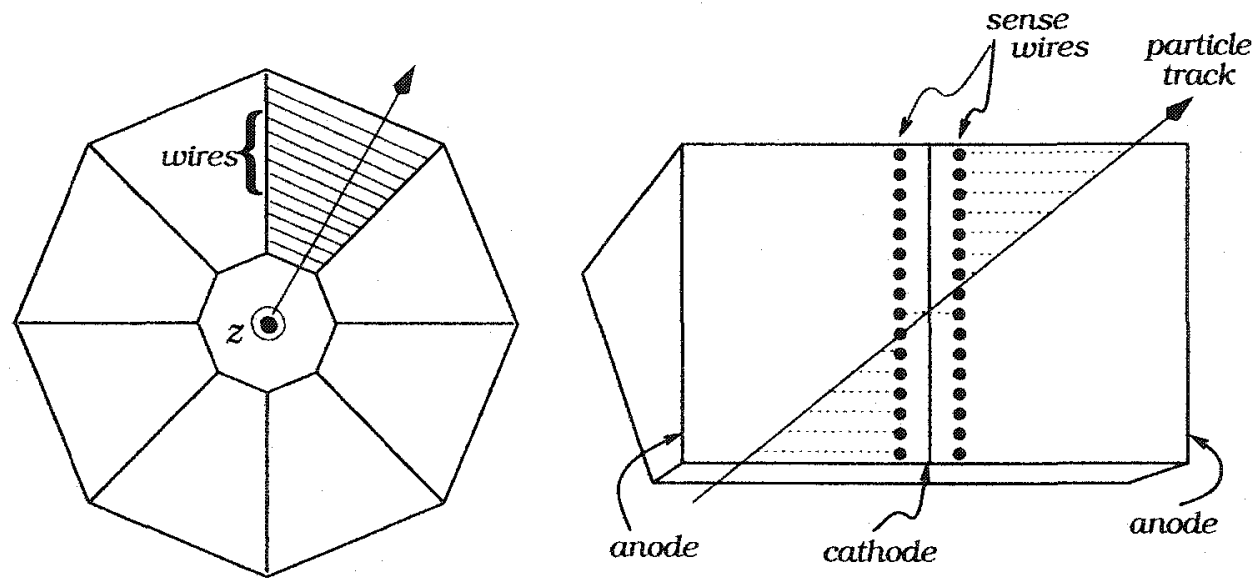

Figure 2.8: A figure of a particle leaving behind an ionization trail as it transverses the VTX. The electrons from the ionization trail are drawn to the sense wires for readout.

to within a resolution of $2.0 \mathrm{~mm}$. Furthermore, identification of multiple vertices can help correct the calorimetry for multiple interactions, and the $z$-vertex information obtained is used to calculate the polar production angle $\theta$. The $z$-vertex calculated by the VTX is also a seed in the pattern recognition software which matches hits in the other tracking chambers. Even in the latter stages of the analysis, many variables depend on the event vertex, such as lateral displacement $\left(L_{x y}\right.$ in Figure $1.7)$.

The VTX detector is located just outside the SVX and extends to an outer radius of $22 \mathrm{~cm}$. With 28 side-by-side modules, each $9.4 \mathrm{~cm}$ long, encircling the beam line as a chain of octant-shaped annuluses. Each chamber is split in half by a voltage plane to reduce the drift distance of charge particles in the $50 \%-50 \%$ Argone-Ethane gas mixture that has a drift velocity of $46 \mu \mathrm{m} / \mathrm{ns}$. As a particle travels through the module, the gas is ionized, and the ions travel parallel to the $z$ axis until a sense wire is hit. Signals can then be read out and amplified to measure the $r-z$ of a charged particle. To get rudimentary $r-\phi$ measurements, each octant 
is rotated $\pm 15^{\circ}$ with respect to each adjacent module. In this manner, a charged track can be isolated to be within $\frac{1}{8}^{\text {th }}$ of an octant. The VTX covers $\left|\eta_{\text {detector }}\right|<3.5$.

The hits of the charged particles are entered into the pattern recognition software to form tracks segments which are extrapolated to the beam-line to yield a primary $z$-vertex measurement. The quality of a vertex depends on the number of hits and the number of track segments used in the pattern recognition. The resulting graded scale of classes of vertices are used to rank a vertex with respect to others. For example, a class 12 vertex is the highest quality vertex with more than 180 hits [6] [7] [8] [35].

\section{Central Tracking Chamber (CTC)}

Surrounding both the SVX and the VTX is an open cell drift chamber called the Central Tracking Chamber (CTC). The purpose of the CTC is to obtain the information necessary for the reconstruction software to precisely measure the 3Dtracks within $\left|\eta_{\text {detector }}\right|<1.1$. The $3.2 \mathrm{~m}$ long cylinder has an inner radius of $0.28 \mathrm{~m}$, and the detector extends out to $1.38 \mathrm{~m}$. There are 30,504 gold-plated tungsten wires grouped into 84 layers. Furthermore, these 84 layers are grouped into 9 superlayers, 5 axial superlayers which are parallel to the beamline as well as 4 stereo superlayers that are $\pm 3^{\circ}$ from the beamline. Each axial superlayer has 4,392 wires (12 sense wire layers), and each stereo superlayer has 1,764 wires (6 sense wire layers). The axial layers yields $r-\phi$ values and the stereo layers measures $r-z$ so together both extract information used to calculate the helixes of many charged particles.

The detector is also subdivided azimuthally into cells by stainless steel HV fieldshaping wires. Potential wires alternate with the sense wires inside each cell. Consequently, there is a $1350 \mathrm{~V} / \mathrm{cm}$ electric field in the CTC in addition to a $1.41 \mathrm{~T}$ magnetic field from the solenoid. 


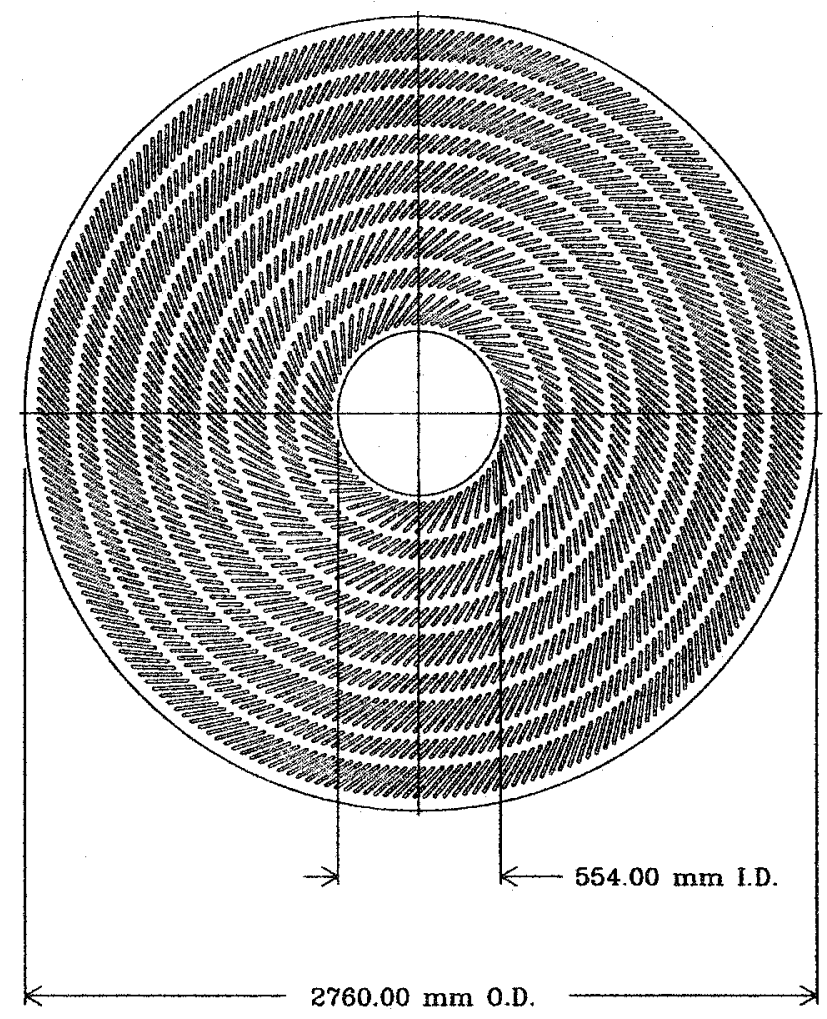

Figure 2.9: A figure of the endplate of the CTC. There are 9 tilted superlayers of sense wires. 5 superlayers measure $r$ - $\phi$, and 4 superlayers measure $r-z$.

The entire chamber volume is filled with a 50/50 Argon-Ethane gas with trace amounts of isopropyl alcohol that has a drift velocity of $46 \mu \mathrm{m} / \mathrm{ns}$. The drift velocity of the gas dictates the cell size because the CTC recovery time $(0.8 \mu s)$ must be less than the beam crossing time in the Tevatron $(3.5 \mu s)$. Otherwise, data from the previous events would still be present in subsequent events.

As a charged particle passes through the CTC, the gas becomes ionized so that electrons drift to the sense wires within 706 ns (or a maximum drift distance of 40 $\mathrm{mm}$ ). Using this hit information as well as primary vertex information from the VTX, the reconstruction code calculates the 5 tracking parameters of a given helix: 
$z_{0}, \phi_{0}, \theta_{0}$, impact parameter $d_{0}$, and the radius of curvature $\mathrm{R}$ in the transverse plane. In particular, the hit information from the axial layers determine $r-\phi$ measurements. Then, the algorithm uses axial hits and the primary vertex, as measured by the VTX, to reconstruct 3D tracks.

In Figure 1.9, the rotation of the cell structure by $45^{\circ}$ appears odd; however, there are motivations for this. Because of the $\mathrm{E}$ and $\mathrm{B}$ fields crossing, the electrons will drift at a Lorentz angle of $45^{\circ}$ from the radial direction. By rotating the cells by this Lorentz angle, the drift time of the electrons is proportional to drift distance to the wire plane. In addition, for any given hit on a sense wire, there is an ambiguity in the drift direction of the electrons. This "ghost" track can be distinguished from the true trajectory by observing that the true path passes through the primary vertex. The rotation accomplishes the same purpose by simply matching segments across superlayers.

The CTC has an azimuthal spatial resolution of approximately $200 \mu \mathrm{m}$ and a spatial resolution of $6 \mathrm{~mm}$ in the axial direction. Momentum measurement resolution is

$$
\frac{\delta p_{T}}{p_{T}} \sim 0.002 p_{T}
$$

if only the CTC is used, and when the CTC is used in conjunction with SVX, the resolution is

$$
\frac{\delta p_{T}}{p_{T}} \sim 0.0009 p_{T}
$$

where $p_{T}$ is given in $\mathrm{GeV} / \mathrm{c}$.

In summary, a charged track passing through the CTC ionizes the gas enclosed. The electrons from the ionized gas drift to sense wires, and the drift time is measured with electronics. These hit times are used to yield helix trajectories. It is interesting 




Figure 2.10: A single wedge of the CEM. Lead-scintillator sandwich converts initial particle energy into light which is collected by the waveguides and transferred to the phototubes.

to note that $K_{S}$, being neutral particles, are not detected directly, but through their charged decay products. This analysis depends heavily on the CTC [6] [7] [8] [35]. 


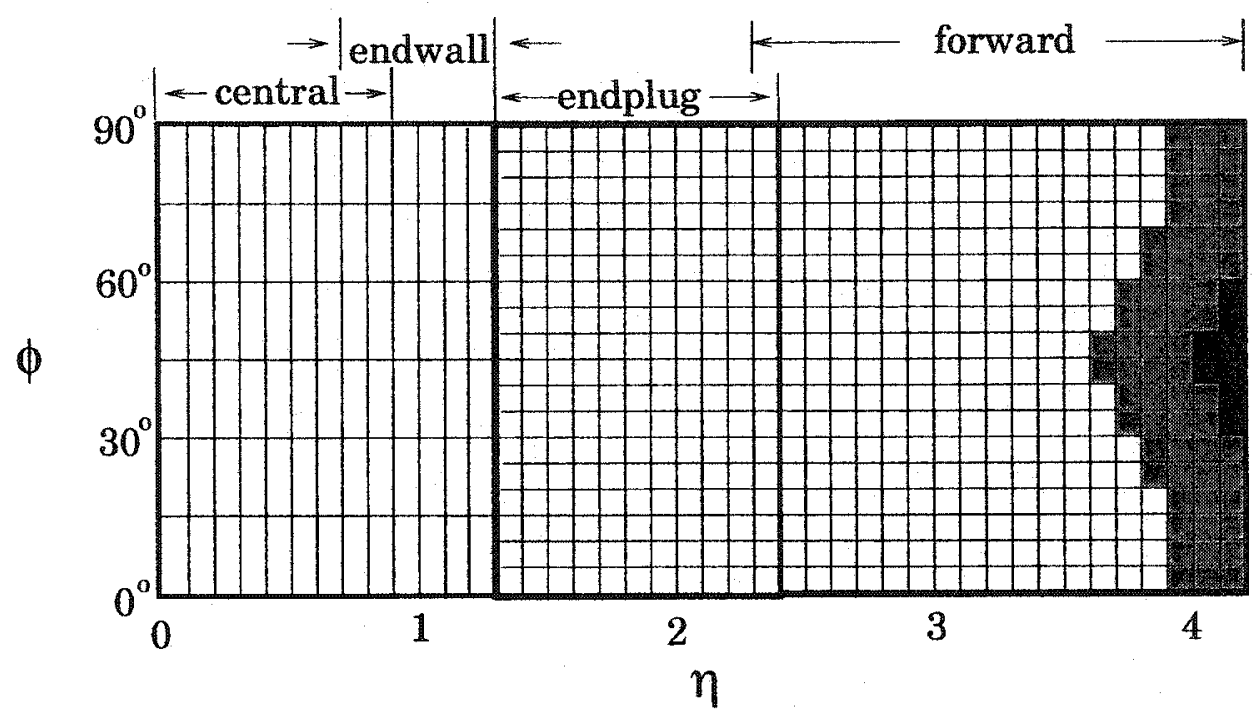

Figure 2.11: A quadrant of the calorimeters showing the $\eta$ - $\phi$ tower segmentation. EM calorimeters have coverage of $0<\eta<4.2$. The area shaded in gray denotes to regions where the HAD has partial coverage due to the low $\beta$ magnets. The black region is where there is no coverage because the beampipe is positioned here.

\subsubsection{Calorimetry}

There are several electromagnetic and hadronic calorimeter subsystems that cover different regions of pseudorapidity. Each is designed to measure the energy of electrons, photons, and hadrons. By causing either electromagnetic or hadronic showers with absorbers and converting particles into light with scintillators, the light that is collected with waveguides and is carried to the phototubes is proportional to the energy of the incident particle. The segmentation of the detector has almost complete azimuthal coverage and about $\left|\eta_{\text {detector }}\right|<4.2$ coverage in pseudorapidity. Only "cracks", areas of the calorimeters without electronic readout, at every $15^{\circ}$ in $\phi$ and at $\left|\eta_{\text {detector }}\right|<0.13,1.1<\left|\eta_{\text {detector }}\right|<1.4$, and $2.3<\left|\eta_{\text {detector }}\right|<2.4$, are insensitive to incident particles. These cracks usually coincide with adjacent detector cells or detector subsystems. Detection and formation of energy clusters is crucial 


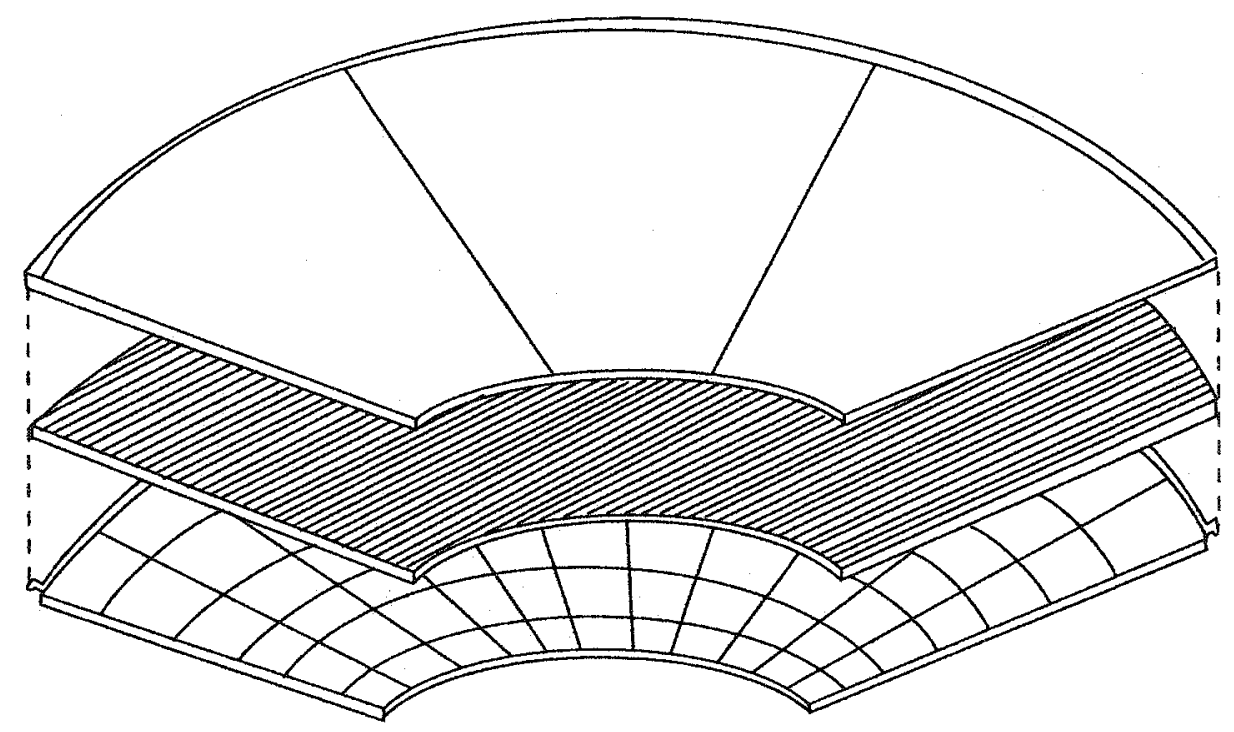

Figure 2.12: A diagram of a quadrant of the PEM where the top layer is that of proportional tubes, the middle layer is that of a lead absorber, and the bottom layer shows the copper etching which gives rise to the segmentation of the cathode pads in $\eta$ (radial lines) and $\phi$ (arcs).

in the identification of jets. The central calorimeter and strip chambers, the plug calorimeters, and the forward calorimeters will all be discussed.

Central Calorimeter, Central Electromagnetic Strip Chambers, Wall Calorimeters (CEM, CES, WHA)

The Central Electromagnetic Calorimeters (CEM) consists of 4 modules, and each pair surrounds both the solenoid and the central tracking systems on either side of the nominal interaction point, $z=0$. Each modular pair has 24 wedges, every $15^{\circ}$ in $\phi$, and each wedge has 10 projective towers, every 1 in $\eta$. The projective tower property of the calorimeters refers to each tower pointing back to the interaction region. The entire CEM is $5.0 \mathrm{~m}$ long and $35 \mathrm{~cm}$ thick, and its volume consists of 31 alternating layers of $3.175 \mathrm{~mm}$ thick lead absorbers and $5 \mathrm{~mm}$ thick layers of 
scintillator. There are 2 wavelength shifters per tower that guides green waveshifted light $(490 \mathrm{~nm})$ to the phototubes. Hence, when a high $E_{T}$ photon or electron hits a lead absorber, the particle interacts with shell electrons to yield Bremsstrahlung $\gamma$ 's. These high energy $\gamma$ convert to electron-positron pairs. The electromagnetic shower cycle continues until all the initial energy of the parent is transferred to $e^{+} e^{-}$ pairs. The scintillating layers convert these electrons and positrons into light which is directed by the waveguides to the phototubes. Overall, the CEM is 18 radiation lengths thick.

Between the $8^{\text {th }}$ lead layer and the $9^{\text {th }}$ scintillator layer, the Central Electromagnetic Strip Chamber (CES) is situated. The location of the CES marks the average maximum transverse depth of an electromagnetic shower, 5.9 radiation lengths into the CEM. The CES is able to measure the position and transverse shower distributions of electromagnetic clusters in $z$ and $r-\phi$ with a detector which is part strip chamber and part gas proportional chamber. The strip chamber consists of 128 cathode strips that are oriented perpendicular to the beam and 64 anode wires which lie parallel the beam. The gas proportional chamber is filled with a $95 \%-5 \%$ Ar- $\mathrm{CO}_{2}$ mixture, and its wires are separated by $2 \mathrm{~mm}$. There is a $1420 \mathrm{~V}$ potential applied to the wires. The position measurement resolution of CES is $2 \mathrm{~mm}$. The CES is particularly useful in identifying $\gamma$ in a electromagnetic shower by studying the shower profile obtained from the strip chamber.

Encompassing the CEM, the Central Hadronic Calorimeter and the Wall Hadronic Calorimeter absorb hadrons, create hadronic showers, and in principle, are very similar to the CEM. Each projective tower of the CHA and WHA corresponds to a tower in the CEM with the pseudorapidity coverage being $\left|\eta_{\text {detector }}\right|<0.9$ for the CHA and $1.7>\left|\eta_{\text {detector }}\right|>1.3$ for the WHA. The CHA consists of 32 layers of 
Table 2.2: Summary of the CDF calorimeters characteristics [7].

The table shows for every calorimeter system, the pseudorapidity coverage, the average energy resolution with energy dependent and systematic uncertainties added in quadrature as calculated directly from electron (for EM) and pion (for HAD) test beam data, the position resolution, and its thickness in term of the radiation lengths (EM calorimeters) or interaction lengths (HAD calorimeters).

\begin{tabular}{|l|r|c|c|c|}
\hline $\begin{array}{c}\text { Calorimeter } \\
\text { subsystem }\end{array}$ & $\begin{array}{c}|\eta| \\
\text { coverage }\end{array}$ & $\begin{array}{c}\text { Energy resolution } \\
\sigma(E) / E\end{array}$ & $\begin{array}{c}\text { Position } \\
\text { resolution } \\
\left(\mathrm{cm}^{2}\right)\end{array}$ & Depth \\
\hline CEM & $0-1.1$ & $13.5 \% / \sqrt{E_{T}} \oplus 1.7 \%$ & $0.2 \times 0.2$ & $18 X_{0}$ \\
CHA & $0-0.9$ & $75 \% / \sqrt{E_{T}} \oplus 3 \%$ & $10 \times 5$ & $4.5 \lambda_{0}$ \\
WHA & $0.7-1.3$ & $75 \% / \sqrt{E_{T}} \oplus 3 \%$ & $10 \times 5$ & $4.5 \lambda_{0}$ \\
\hline PEM & $1.1-2.4$ & $28 \% / \sqrt{E_{T}} \oplus 2 \%$ & $0.2 \times 0.2$ & $18-21 X_{0}$ \\
PHA & $1.3-2.4$ & $130 \% / \sqrt{E_{T}} \oplus 4 \%$ & $2 \times 2$ & $5.7 \lambda_{0}$ \\
\hline FEM & $2.2-4.2$ & $25 \% / \sqrt{E_{T}} \oplus 2 \%$ & $0.2 \times 0.2$ & $25 X_{0}$ \\
FHA & $2.3-4.2$ & $130 \% / \sqrt{E_{T}} \oplus 4 \%$ & $3 \times 3$ & $7.7 \lambda_{0}$ \\
\hline
\end{tabular}

steel absorber interleaved with $1.0 \mathrm{~cm}$ thick plastic scintillator. The WHA has 15 layers of $5.1 \mathrm{~cm}$ steel absorbers with alternating layers of $1.0 \mathrm{~cm}$ thick plastic scintillator. The extra thickness in the WHA is due to the fact that a particle will on average have 1.4 times more total energy in the WHA than in the CHA for a given $E_{T}[6][7][8][35]$.

\section{Plug Calorimeters (PEM/PHA)}

Enclosing the ends of the conical volume defined by $\theta=30^{\circ}$ left by the WHA, two separate plug calorimeters fit snuggly. Each endcap consists of $490^{\circ}$ azimutha quadrants with a $10^{\circ}$ hole to permit the beam pipe to run through the center. The radius of a quadrant is $1.4 \mathrm{~m}$. The plug electromagnetic calorimeter is nearest to the interaction point and is $50 \mathrm{~cm}$ deep, and the plug hadronic calorimeter is just beyond. Both PEM and PHA have readout cathode pads etched in a projective geometric scheme, having a segmentation of $5^{\circ}$ in phi and 0.09 in $\eta$. However, the 
pads near the shower max have 4 to 5 times more segmentation to allow a more precise measurement of the profiles of showers. The PEM has 34 layers of gas proportional tubes sandwiched with $2.7 \mathrm{~mm}$ thick lead absorbers, and the PHA has 20 layers of proportional tubes intermixed with $5.1 \mathrm{~cm}$ thick steel absorbers. Each tube is composed of conductive plastic and is filled with a 50\%-50\% Argone-Ethane gas mixture. For proportional tubes, the quantity of charge collected on the wires is directly proportional to the ionization of the gas which is itself proportional to the initial energy of the hadron. The PEM has a coverage of $1.1<\left|\eta_{\text {detector }}\right|<2.4$ whereas the PHA has a coverage of $1.32<\left|\eta_{\text {detector }}\right|<2.4$. Energy and position resolutions of all the calorimeters are listed in Table 2.1 [6] [7] [8] [35].

\section{Forward Calorimeters (FEM/FHA)}

To include the $2.2<\left|\eta_{\text {detector }}\right|<4.2$ projective geometric region, FEM and FHA detectors enclose the ends of the cylindrical portion of the CDF detector, enabling measurements of the energies of particles to be found as close as $2^{\circ}$ from the beamline. The tower segmentation of both the FEM and FHA are 0.1 in $\eta$ and $5^{\circ}$ in $\phi$, in this high $\eta$ region. The $1.0 \mathrm{~m}$ deep FEM is $6.5 \mathrm{~m}$ from the center of the detector, with each side $3 \mathrm{~m}$ in length. In the FEM, there are 30 sampling lay ers of proportional tube chambers alternating with $0.48 \mathrm{~cm}$ thick layers of $94 \%-6 \%$ Lead-Antimony alloy absorbers. The FHA, located just behind the FEM, has 27 sampling layers of proportional tube chambers with $5.1 \mathrm{~cm}$ thick steel absorbers interspersed. Since the eta region of interest is $\left|\eta_{\text {detector }}\right|<1.0$ in this analysis, the forward and plug calorimeters are not very useful [6] [7] [8] [35]. 


\subsubsection{Muon Detector}

The Central Muon Chambers (CMU), the Central Muon Upgrade Chambers (CMUP), the Central Extension Muon Chambers (CMX), and the Forward Muon System (FMU) provide muon detection. If a muon track can be associated with a low energy cluster in the calorimeter, a muon can be identified. The muon chambers are proportional chambers which detect tracks which traverse the steel filters, the central tracking systems, the calorimeter systems, and the solenoid. Even a muon with a $p_{T}$ as low as $1.5 \mathrm{GeV} / \mathrm{c}$ can pass through all other parts of the detector (3.487 $\mathrm{m}$ from the beam axis), depositing little energy in the calorimeters. The number of interaction lengths of steel are 5,8 , and 6 between the beam axis and the CMU, CMUP, and CMX, respectively. The $\phi$ coverage is $85 \%, 80 \%$, and $67 \%$ for the CMU, CMUP, and the CMX, and the $\eta$ coverage is $\left|\eta_{\text {detector }}\right|<0.6$ for the combined CMU and CMUP systems, $0.6<\left|\eta_{\text {detector }}\right|<1.0$ for the CMX, and $2.0<\left|\eta_{\text {detector }}\right|<3.6$ for the FMU. The CMU and CMUP systems are steel filters in front of 4 layers of drift chambers, and the CMX two pairs of arches with drift chambers located behind the central and wall calorimeters. The FMU system is a pair of magnetized iron toroids (used also as steel filters) in which the drift chambers and scintillators for the toroid instrumentation are also used for muon measurements [6] [7] [8] [35].

\subsubsection{Beam-Beam Counters}

Mounted on the front of the forward calorimeters, there four scintillating counters confined to the same plane on opposite sides of the beam pipe, $5.4 \mathrm{~m}$ from the interaction point. The coverage is $3.2<\left|\eta_{\text {detector }}\right|<5.9$. If both BBC counters have coincident hits within $15.0 \pm 0.2$ ns of a bunch crossing, then a legitimate $p \bar{p}$ collision is believed to have occurred. This provides a veto for beam interactions with 


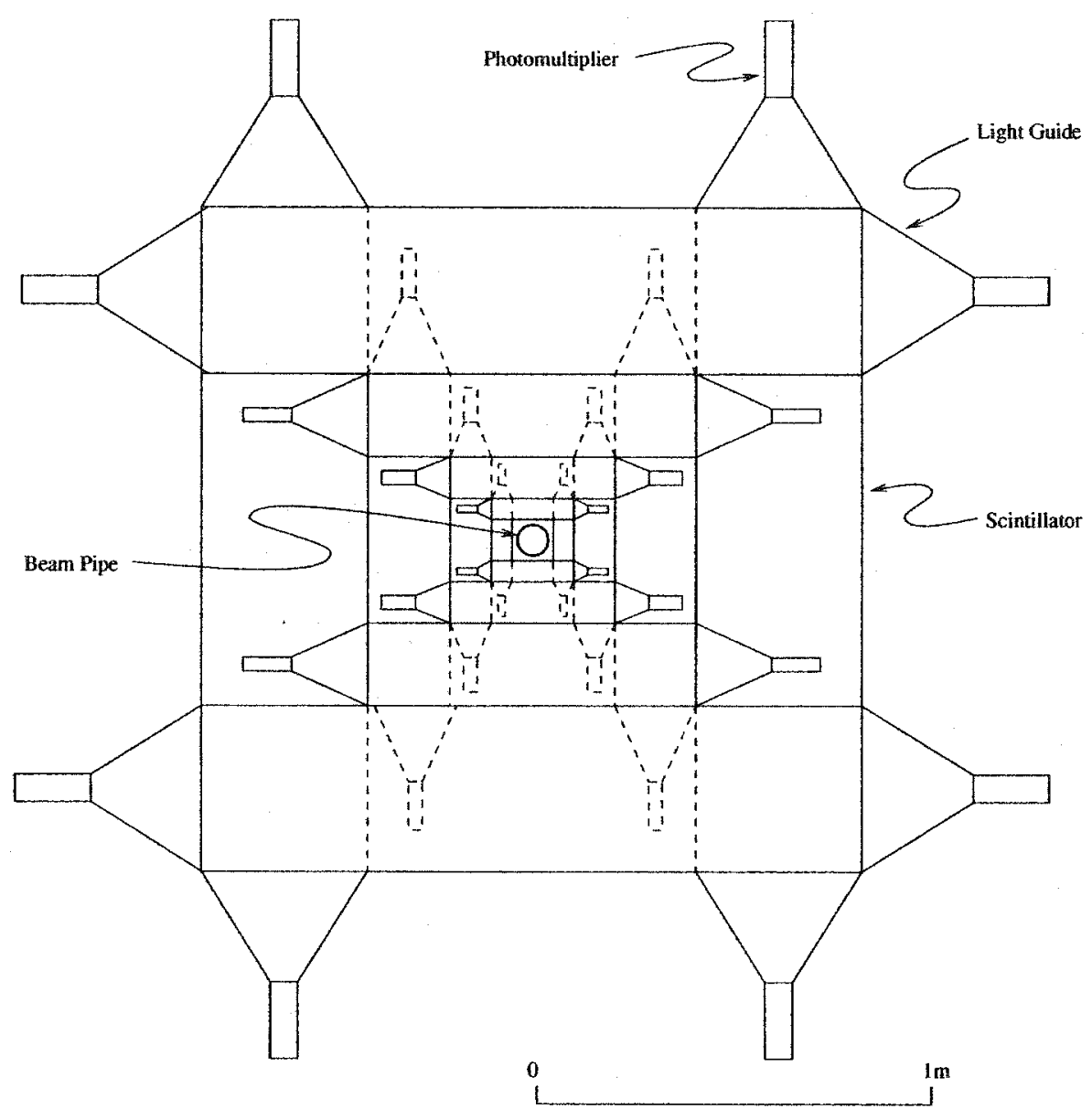

Figure 2.13: A diagram of one set of Beam-Beam Counters (BBC).

beam-pipe gas. Furthermore, successful readout from the beam-beam counters is equivalent of passing the Minimum Bias Trigger. Also, instantaneous luminosity is derived from the quotient of the rate of coincident hits and the effective $\sigma_{B B C}$. In this analysis, the track embedding sample used the same instantaneous luminosity distribution as the data to prevent skewed efficiencies [6] [7] [8] [35]. 


\subsection{Data Acquisition and Trigger Systems}

When the 6 proton and 6 antiproton bunches collide every $3.5 \mu s$ (or at frequency of $285 \mathrm{kHz}$ ) with an average of $3.0 p \bar{p}$ interactions per bunch crossing for Run $1 \mathrm{~B}$, there can be several hundred thousand interactions per second of which only a few events per second can be written onto tape. Most of these events are diffractive events whereas the events of most interest are usually hard-scattering events; hence, there are a series of hardware and software event cuts that reject "uninteresting" events at rates of $10^{4}-10^{5}$ while retaining most of the "interesting" events. For example, a $t \bar{t}$ event ( $\sigma$ of about $5 \mathrm{pb}$ ) occurs once every 5 hours and an inclusive $W$ boson event ( $\sigma$ of $25 \mathrm{nb}$ ) occurs once every 4 seconds, and these events are not the ones you want to throw away with the other $10^{4}-10^{5}$ "uninteresting" events every second. To accomplish this feat, each trigger stage has stricter event criteria which allows fewer events for longer processing during the next stage. It is important to make decisions and readout data quickly in order to reduce the amount of time which the Data Acquisition System (DAQ) cannot readout data (dead time). There are 3 general types of triggers for an event to pass in order to be written to tape. They are called the Level 1, Level 2, and Level 3 triggers.

\subsubsection{Level 1 Trigger}

The Level 1 Trigger is a hardware trigger which evaluates every event before the next beam-crossing, within $3.5 \mu s$. Without any deadtime, it brings the event rate from several hundred thousand per second to only a few $\mathrm{kHz}$. This trigger selects mostly hard scattering events by making sure there are hits in the $\mathrm{BBC}$ counters and either basic energy deposition in a calorimeter or hits in the muon chambers. Only $1-2 \%$ of the events are passed onto the L2 trigger [6] [7] [8] [35]. 


\subsubsection{Level 2 Trigger}

The Level 2 trigger decision process takes longer than the bunch-crossing frequency, 25-35 $\mu s$, and there is not an option to buffer the data (storage of information for latter processing). Hence, the next 7-10 bunch crossings are ignored upon a Level 1 accept, and few percent of deadtime is a result. Tracking, photon, jet, electron, and other higher order objects are processed at this stage, and upon a L2 accept, the data from the detector is digitized by the Front-end electronics and readout by scanners with the aid of a data acquisition system. This process takes $3 \mathrm{~ms}$, during which the DAQ is unable to take data, and this is responsible for another few percent of deadtime. The Trigger Supervisor checks the digitization of the event while the Event Builder assembles digitized data into an event structure. Events are passed onto the $\mathrm{L} 3$ trigger at rates of $22 \mathrm{~Hz}$ for Run $1 \mathrm{~A}$ and $44-55 \mathrm{~Hz}$ for Run 1B. However, for some types of events, in which more events pass than can be processed by Level 3, only a fraction of events are taken, and the resulting sample is prescaled [6] [7] [8] [35].

\subsubsection{Level 3 Trigger}

The Level 3 trigger buffers and processes events in parallel without deadtime using only software triggers. It uses simpler and quicker versions of tracking algorithms in order to fully reconstruct electron, jet, muon, and photon objects, and if it passes the designed L3 trigger, the data is then written to staging disks and latter stored onto $8 \mathrm{~mm}$ tape at a rate of up to $5-7 \mathrm{~Hz}$ for Run $1 \mathrm{~A}$ and $10 \mathrm{~Hz}$ for Run $1 \mathrm{~B}$. The L3 trigger system employs 48 parallel CPU's which can operate with the computing power of 1000 MIPs (Millions of Instructions Per second).

This analysis depends primarily on the minimum bias triggers and jet triggers, 
which will be discussed in more detail in the next two subsections. In addition, due to acceptance and detector efficiencies, even a smaller fraction of events that pass the triggers are studied [6] [7] [8] [35].

\subsubsection{Minimum Bias Triggers}

The run $1 \mathrm{~B}$ minimum bias data stream, XMBB5P, has coincident hits (within 15.0 $\pm 0.2 \mathrm{~ns}$ of a beam crossing) in the BBC counters [7] [36] [37]. This is the only hardware requirement for a minimum bias event. Other event selection criteria will be discussed in the minimum bias selection chapter.

\subsubsection{Jet Triggers}

The jet data sample is taken from 4 different types of tape sets: QJ2B_P, QJ5B_P, QJ7_P, and QJ0_P. Events on the QJ2B_P tapes must pass the JET_20_TEX_0_PRE_25_V2 Level 2 trigger and the QCDB_JET4_20_TEX_0 Level 3 triggers. First, the event must pass L1_4_Prescale_40 Level 1 trigger that prescales the sample by a factor of 40 . Then, at least one jet at Level 2 must have at least $20 \mathrm{GeV}$ as well as have more than $0.5 \mathrm{GeV}$ on transverse energy for each electromagnetic tower (JET_20_TEX_0_PRE_25_V2 trigger). The prescale at this stage is 25 . Finally, after the Level 3 jet clustering algorithm, at least one jet with at least $10 \mathrm{GeV}$ must be present to pass the QCDB_JET4_20_TEX_0 Level 3 trigger.

The QJ5B $\mathrm{P}$ events must pass the same Level 1 prescale trigger, the Level 2 clustering algorithm must have at least one jet above $50 \mathrm{GeV}$ (JET_50_V3 trigger), and the Level 3 clustering algorithm must have at one jet with at least $35 \mathrm{GeV}$ (QCDB_JET3_50 trigger). The QJ7PP events must pass the JET_70_V3 Level 2 trigger and the QCDB_JET3_70 Level 3 trigger. An event must have at least one 
jet with at least $70 \mathrm{GeV}$ at Level 2 and at least one jet having at least one jet above $70 \mathrm{GeV}$. A QJ7B.P event is prescaled by a factor 8 at Level 2 .

The QJ0B_P events actually consist of 2 non-prescaled Level 3 triggers: QCDB_JET1_100 and QCDA_JET_140. The first must have at least one jet (after the Level 2 Jet_100_V13 trigger) above $80 \mathrm{GeV}$, and the second must have at least one jet have at least $140 \mathrm{GeV}$ and pass an online cosmic filter (note this a different cosmic filter than one described below). As for the Level 2 Jet_100_V13 trigger, at least one jet must be above $100 \mathrm{GeV}[36][37]$. 


\section{Chapter 3}

\section{Minimum Bias: The Selection Criteria}

As mentioned in the previous chapter, the Minimum Bias events have coincident hits in the BBC counters. As a consequence of such a wide acceptance criteria, the odds that any given Minimum Bias event contains either $W$ bosons, $Z$ bosons, or high energy jets is remote. Moreover, the Minimum Bias events also have a lower track multiplicity than the events which pass one of the jet triggers. Consequently, this permits $K_{S}$ to be examined in an isolated environment, and the knowledge attained here can later be employed to the more complicated jet events. Upon contrasting $K_{S}$ properties and production to earlier publications, the focus will return to the primary purpose of this analysis which is the production of $K_{S}$ in jets. In the first of three chapters having to do with $K_{S}$ found inside Minimum Bias events, the event and track selection criterion, the method of identifying both primary and secondary vertices, and a description of the cuts necessary for the isolation of $K_{S}$ will all be discussed. 


\subsection{The Event Selection Criteria}

The Run 1B Minimum Bias data stream, XMBB5P, is run through and about 1.4 million events are processed. Aside from having coincident hits (within $15.0 \pm 0.2$ ns of a beam crossing) [7] in the BBC counters, each Minimum Bias event satisfies certain criteria such as pass the cosmic filter, "bad run", and primary vertex cuts.

Every event must first pass a cosmic filter that vetoes events with more than $6.0 \mathrm{GeV}$ of "out-of-time" energy. "Out-of-time" energy refers to a cluster in the hadronic calorimeters that is outside a timing window of the CTC for a given event; this "out-of-time" energy is not restricted to eliminating cosmic events but also main-ring splash events, monojet events, and dijets with small missing $E_{T}$. In looking at 8669 minimum bias events, all but one event passes the cosmic filter. The cosmic filter has much more of an effect with the jet events and will be discussed later [38] [39].

Each event must also meet certain criteria in order to be considered a good event which is acceptable for study. First, the delivered integrated luminosity must be greater than $1.0 \mathrm{nb}^{-1}$ with stable beams conditions. The solenoid, the temperatures, the voltages, the trigger rates, and the electronics must be within design specifications. In addition, the offline data is analyzed to check for unacceptable detector behavior that would indicate a problem with the data. The events that meet these requirements are said to have passed "bad run" cuts [7].

Finally, the leading primary vertex must be contained within $60.0 \mathrm{~cm}$ from the $z=0$ of the detector in order to ensure that the particles of an event are in the region where the CDF detector is most efficient [7]. In Table 3.1, the number of events after each event cut is listed along with the $K_{S}$ efficiency. Only approximately $8 \%$ of the $K_{S}$ in the data are lost after these event cuts. 
Table 3.1: The number of Minimum Bias events after each successive cut.

\begin{tabular}{|c|c|c|}
\hline Cuts & $\begin{array}{c}\text { Number of } \\
\text { Minimum Bias Events }\end{array}$ & Fraction of Events Remaining \\
\hline Only Cosmic Filter & $1,381,935$ & 1.000 \\
Bad Run & $1,379,960$ & 0.999 \\
$\left|z_{\text {primary }}\right|<60.0 \mathrm{~cm}$ & $1,272,470$ & 0.921 \\
\hline
\end{tabular}

\subsection{The Track Selection Criteria}

Track must first be reconstructed from hits in the CTC with the pattern recognition software. The software starts by combining the hits within each superlayer, fitting the axial hits to a circle and the stereo hits to a line. The stereo fit takes the $z_{\text {vertex }}$ from the VTX as the input, and in this manner, the 3D track efficiencies are dictated by both the resolutions of the VTX and the CTC.

Once the axial "segments" are found for each superlayer, two independent algorithms work to match segments across superlayers to find 2D tracks. Whenever track results from the two algorithms are unique, the $2 \mathrm{D}$ track solution is combined with the stereo fit to calculate a $3 \mathrm{D}$ track. If the two algorithms yield two solutions with hits in common, the $2 \mathrm{D}$ track that has the most hits in the fit will be passed along for $3 \mathrm{D}$ fitting. However, if the two algorithms yield two solutions with overlapping hits with the same number of hits in each fit, then one $2 \mathrm{D}$ track is chosen at random for $3 \mathrm{D}$ fitting.

In particular, a segment within a given cell must have at least 5 hits and pass through the sense wire plane. To match segments across cells, 8 hits have their residuals (the absolute value of the difference between the fit values and the projected fit values with the hits) less than $500 \mu \mathrm{m}$. These hits are added to the segment, and this new segment is used as a seed for further calculations. The resulting arc 
or circle is fit to a straight line that must pass through the beam position. The hits within a $2 \mathrm{~mm}$ "road" about a circle are added to the fit, another circular fit is done, and the process repeats. The calculation terminates if either there are 3 wire layers between the beam axis and the seed segment or until the beam is reached. The best $\frac{3}{4}$ of residuals are averaged and the hits which are 3 times this average are removed, and then the circle fit is performed again. There is one caveat. If a fit has 20 hits without any of the hits below superlayer 3 , the process is repeated without the beam constraint. The result of this procedure is a $2 \mathrm{D}$ track ready for 3D fitting. The $r-\phi$ of the nearest circle to the beamline and the $z_{\text {vertex }}$ from the VTX is combined to form a trial trajectory. Stereo hits within $1.5 \mathrm{~mm}$ of the trial trajectory are added, and the trial trajectory is recalculated, again with the hits with large residuals removed. In the end, a 3D track defined by 5 track parameters is found [40] [41].

A 3D track is a helical trajectory of a charged particle in a constant magnetic field with the axis of the helix along the solenoidal field. On an event display, looking down the beampipe, the arcs and the circles are the tracks (solid lines) derived from the hits (dots). The larger the transverse momenta of the charged particle, the less the magnetic field will deflect its trajectory and the straighter the path. With the lower momenta tracks (below $400 \mathrm{MeV}$ ), some of the low momenta charged tracks can spiral inward and do not pass enough CTC superlayers to be reconstructed. The neutral particles pass straight through the CTC undetected [41].

There are 5 parameters that define a helix, and hence a 3D track: $d_{0}$ (the impact parameter), $c$ (the signed half-curvature), $\phi$ (the angle between the closest approach and the $x$-axis), $\cot \theta$ (the cotangent of the spiral angle), and $z_{0}$ (the closest approach to the beamline). Figure 3.1 illustrates a few of them in the $r-\phi$ plane. Although 
all of the parameters are indispensable, the impact parameter and the curvature usually have additional cuts further along the analysis process. First, a cut on the impact parameter is incorporated in quite of few analyses since the prompt tracks have impact parameters very close to the primary vertex whereas secondary tracks have impact parameters that are further away from the origin. For $K_{S}$ production, the tracks are typically displaced from the origin so requiring tracks to exceed some minimum impact parameter value does indeed reduce the background. It is possible to increase the signal to noise ratio where the number of $K_{S}$ is the signal and the number of background candidates is the noise. However, the number of $K_{S}$ is reduced substantially and the impact parameter cut biases the $p_{T}$ of the $K_{S}$ (the greater the $p_{T}$ of the $K_{S}$, the further the tracks are from the primary vertex, and thus, the greater the impact parameter values). As a result, an impact parameter cut is not implemented in this analysis. As for the curvature parameter value, the sign of $c$ indicates the charge of the particle while the magnitude is inversely proportional to the diameter of the projected circle on the plane perpendicular to the beam. The half-curvature is also inversely proportional to the $p_{T}$, and later, there will be track cuts made on the $p_{T}$ of each pion track from the $K_{S}$ in order to optimize the efficiency of the CTC [41] [42].

\subsection{The Primary Vertex Selection Criteria}

As discussed earlier, of up to several primary z-vertices, only a single primary vertex is selected from where to initiate the search for the secondary vertices. Although it is possible to find the secondary vertices without the primary vertices, certain $K_{S}$ variables (to be described later) depend upon identifying the "correct" primary vertex. Finding the "correct" primary vertex is complicated by multiple vertices from 


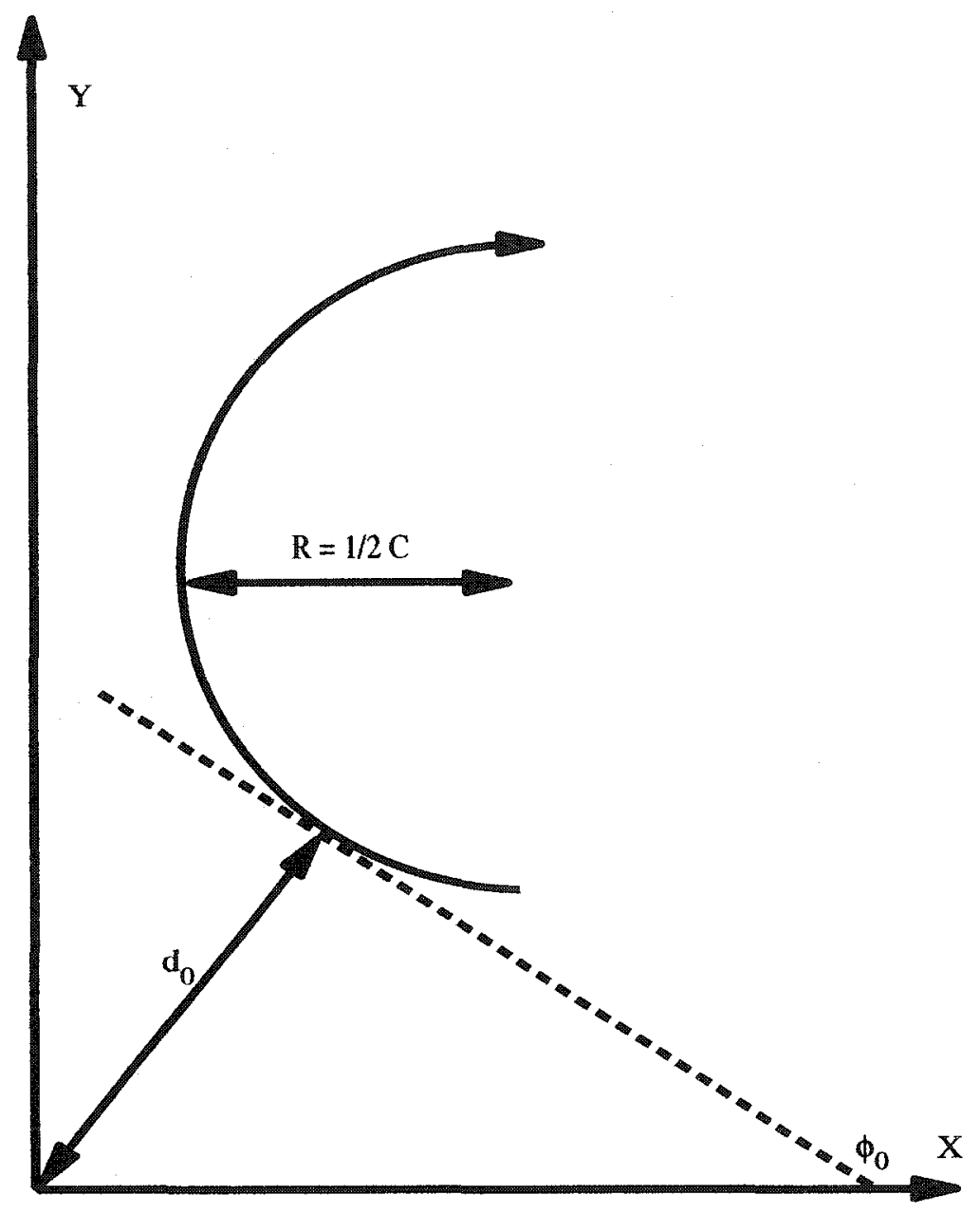

Figure 3.1: The track parameters

any given interaction as well as the multiple vertices from the multiple interactions. Depending upon the analysis, there are different methods of selecting an event ver-tex from a list of primary vertices for each event. Some select only the events with one event vertex [43], and some select the primary vertex nearest to the secondary vertex of the particle of interest [44]. Others correlate certain high momentum objects with the primary vertices, i.e. the $z$-coordinate of either a high $p_{T}$ muon [44], electron [41], or jet [8]. Too many events are lost if the first method is selected. As for the second method, the sample may be biased by selecting only those $K_{S}$ which 


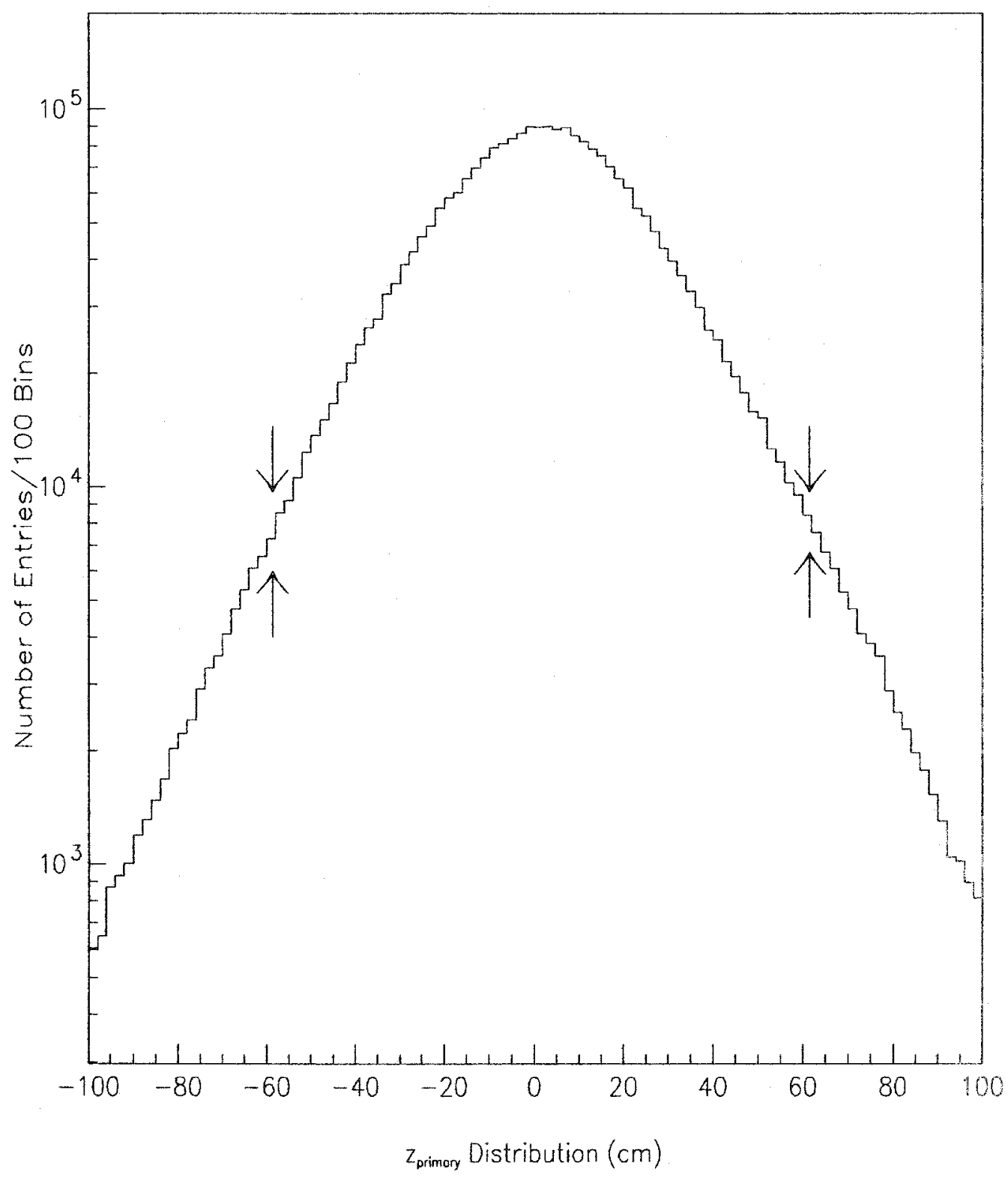

Figure 3.2: The $z_{\text {primary }}$ distribution. The cut of $\left|z_{\text {primary }}\right|<60.0 \mathrm{~cm}$ is used.

decay close to the primary. There are just not enough high $p_{T}$ objects in order to implement the third method without a tremendous loss of Minimum Bias events. 
In this analysis, only the best quality vertex is used for each event, and this is quite common. The quality of a vertex is proportional to the number of hits in the VTX associated with a given vertex [6] [7] [8] [35].

One question which comes to mind immediately is what if the wrong vertex is chosen? The short answer is that a given $K_{S}$ may be found to have a secondary vertex, but the particle is unlikely to point back to the primary vertex. There is a "pointing constraint" in the fitting routine. Whenever a secondary vertex is found, this "pointing constraint" checks to see that it appears to have originated from the selected primary vertex. In particular, this pointing constrait is enforced when a cut is made on the value of the $\chi^{2}$ (the $\chi^{2}$ will be discussed later) [45].

As for the other vertex components, they are considered to be linearly related to the beam spot size and the slope of the beam along the $\mathrm{z}$ direction in many analyses. However, the beam spot size is usually less than $40 \mu \mathrm{m}$ [44], and the $c \tau$ of a $K_{S}$ is on the order of a couple of centimeters [9]; hence, a $v_{x}=v_{y}=0.0$ approximation is sufficient. On the other hand, for B-particles having $c \tau$ of about a few hundred microns [9], this approximation is not good.

\subsection{The Secondary Vertex Selection Criteria}

The secondary vertices are the crux of the analysis. A combined geometric and kinematic fitting routine called CVTMFT is utilized to recognize when a group of tracks originate from a given space point other than the primary vertex. CTVMFT looks for the arcs that intersect at any point in the $x-y$ plane given the track parameters of the potential daughters. Then, the 3D secondary vertex reconstruction incorporates the remaining coordinate information. Other physical constraints, such as either daughter particles having certain masses or the momentum of the parent 
must point back to the primary vertex, are applied in order to reduce the errors of the result.

The best manner to understand how CTVMFT locates the secondary vertices is to discuss an example, $K_{S} \rightarrow \pi^{+} \pi^{-}$. First, the daughter tracks must originate from the same space point. In this case, the pions must originate at the point where the $K_{S}$ decays. If either the particle or its daughters has its mass known, additional mass constraints can be set to find the secondary vertex. Here, the $K_{S}$ decaying into pions is the process sought, so the known masses of the pions is added to the kinematic constraints of the fit. Usually, the daughter masses are fixed and the unknown parent mass is left as a floating value. This is optional in CVTMFT, but this analysis depends on this feature. Also, there exists another optional constraint that the secondary vertex must point back to the primary vertex (or another arbitrary direction). This assumption is reasonable since most $K_{S}$ will be originating directly from the primary vertex or from another particle that points back to the primary vertex. Once the constraints are settled upon, CTVMFT can sort through pairs of tracks to check which meet all the constraints.

The CVTMFT algorithm begins with the initial track parameters of the two tracks and adjusts the track parameters in order to minimize $\chi^{2}$ equations consisting of all constraints applied concurrently. To elaborate, in its simplest form, $\chi^{2}$ is the sum of a set of linear equations that are the functions of the differences between geometric and kinematic values of the tracking parameters as well as the error matrix of these tracking parameters. Other constraints such as a pointing constraint only add to the number of free parameters. In order for $\chi^{2}$ to be minimized, these nonlinear derivative equations of $\chi^{2}$ with respect to every free parameter is equal to zero. These $\chi^{2}$ derivative equations are thus expanded into a Taylor series with only 
Table 3.2: The number of $K_{S}$ after each successive cut. Errors are taken from the fit of the area of the Gaussian.

\begin{tabular}{|c|c|c|}
\hline Cuts & Number of $K_{S}$ & Fraction of $K_{S}$ Remaining \\
\hline Only Cosmic Filter & $125992 \pm 1323$ & $1.063 \pm 0.016$ \\
\hline Badrun & $125991 \pm 1323$ & $1.063 \pm 0.016$ \\
\hline$\left|z_{\text {primary }}\right|<60.0 \mathrm{~cm}$ & $118570 \pm 1291$ & 1.000 \\
\hline$\chi_{K S}^{2}<20.0$ & $95490 \pm 938$ & $0.805 \pm 0.012$ \\
\hline$\left|z_{K S}-z_{\text {primary }}\right|<3.0 \mathrm{~cm}$ & $92186 \pm 911$ & $0.777 \pm 0.011$ \\
\hline$p_{T}^{\text {TracksfromKS }}>300 \mathrm{MeV}$ & $82957 \pm 813$ & $0.700 \pm 0.010$ \\
\hline$\left|\eta^{K S}\right|<1.0$ & $67221 \pm 696$ & $0.567 \pm 0.009$ \\
\hline $3 \mathrm{D}$ Displacement ${ }^{K S}>1.0 \mathrm{~cm}$ & $61562 \pm 562$ & $0.519 \pm 0.007$ \\
\hline $\cos \theta_{P D}{ }^{K S}<0.990$ & $56941 \pm 441$ & $0.480 \pm 0.006$ \\
\hline
\end{tabular}

the linear terms being kept. Then, these linear $\chi^{2}$ equations are solved, and the solutions are substituted again for the generation of a new set of the $\chi^{2}$ derivative equations. Again, the process of Taylor expanding and solving these sets of linear $\chi^{2}$ equations is iterated several times. Convergence of the solutions is not guaranteed. Later, a $\chi^{2}<20.0$ cut is applied in this analysis to reduce the number of diverging fits for $K_{S}$ candidates.

In the end, when a pair of tracks is found to have originated from a secondary vertex, then the adjusted track parameters, the adjusted vertices, and the coordinates of the secondary vertex are returned. It is very important to state that the track parameter values and displacement values are altered by CTVMFT during the secondary fitting process. However, the differences are slight. Most of the candidates CVTMFT keeps are not $K_{S}$, or any real particles for that matter, but rather just background of pairs of tracks. If these adjusted tracks can both be extrapolated back to the secondary vertex, then the mass and the momentum of the parent is calculated [45]. 


\subsection{The $K_{S}$ Selection Criteria}

To reiterate, only the first in a list of primary vertices is considered as the event vertex. By convention, this is the best quality vertex. In addition to the primary vertex, every oppositely charged pair of "good" 3D CTC tracks (with each track $p_{T}$ above $200 \mathrm{MeV}$ ) is put through a V-Finder (CTVMFT) to be considered as candidates for $K_{S}$. A "good" 3D track has either at least 2 axial superlayers with at least 4 hits each and 1 stereo superlayer or some other stringent combination of axial hits and stereo information [46]. The V-Finder checks to see whether a given pair of tracks, whose masses are assumed to be those of pions, intersect within a region of $3 \mathrm{D}$ space and their summed total momentum points back to the primary vertex. In addition, each individual track must not only intersect but also to have originated from the calculated secondary vertex returned by the V-Finder. If these criteria are satisfied, the tracks of the daughter particles are taken by another routine that reconstructs the 4 -vector of the parent particle. If the "parent" mass calculated is within 0.45 and $0.55 \mathrm{GeV}$, approximately $\pm 50 \mathrm{MeV}$ around the mass of a $K_{S}$, then it is booked according to the mass window it falls under and is considered as a valid candidate that can be further studied [41] [43] [47] [48].

These candidates undergo successive cuts in order to increase the signal to noise ratio. The effect of each cut is shown in Table 3.2 , and a description of each cut will be detailed below.

The $\chi^{2}$ Cut

For every fit, a $\chi^{2}$ is returned from the fitting routine, and this value is considered as a check to the accuracy of the fit. The rapidly decreasing tail distribution runs from 0.0 to a number that increases with the added constraints. In particular, the pointing back criteria broadens this distribution because it includes the primary 
vertex errors in this calculation. The $\chi^{2}$ cut is not a particularly useful one; it acts on both the background and the signal in the same manner and does little to significantly enhance the signal to noise ratio. However, a $\chi^{2}<20.0$ does eliminate candidates with extremely bad CTVMFT fits. In the top of Figure 3.3, the cut at $\chi^{2}=20.0$ is denoted by arrows as well as the effect of the reconstructed mass spectrum after the cut [41] [43] [45] [47] [48].

\section{The $\left|z_{K S}-z_{\text {primary }}\right|$ Cut}

The $z_{K S}$ value refers to z-coordinate obtained after extrapolating the momentum of the $K_{S}$ back to the beamline. This value is calculated using a slope equation in the following manner:

$$
z_{K S}=v_{z}^{K S}-v_{x}^{K S} \frac{p_{z}^{K S}}{p_{x}^{K S}} .
$$

The smaller the scalar difference between $z_{K S}$ and the primary vertex, the more likely the $K_{S}$ originates from the primary vertex. In the bottom of Figure 3.3, the difference between the extrapolated $z_{K S}$ and the primary vertex is plotted along with arrows at $\pm 3.0 \mathrm{~cm}$ to indicate the cut values [41] [43] [47] [48].

\section{The Cut on the $p_{T}$ of the Tracks}

Although the CTC can measure the individual track momenta down to $200 \mathrm{MeV}$ and many other studies utilize a $400 \mathrm{MeV}$ track cut, this analysis will implement a $300 \mathrm{MeV}$ track cut. Low momenta tracks, below $400 \mathrm{MeV}$, are more likely to loop in circles inside the CTC rather than emerge from it when compared to charged tracks with higher momenta. Moreover, the lower the track $p_{T}$, the more likely the track will not have as many hits in the CTC. Both of these effects cause not only a decrease in the single track efficiency (and consequently the $K_{S}$ efficiency) but also produces a large charge asymmetry for low momenta tracks. However, the $K_{S}$ have a large dependence on the track $p_{T}$ cut. This is because most $K_{S}$ are generated 
with relatively low $p_{T}$ (below $3.0 \mathrm{GeV}$ ), and since the track efficiencies have a strong track $p_{T}$ dependence, it follows that the $K_{S}$ also have a strong dependence on the track $p_{T}$. Far too many $K_{S}$ would be lost in the Minimum Bias set because the $K_{S}$ are produced more abundantly with lower momenta. In particular, a $400 \mathrm{MeV}$ track cut would have $70556 \pm 827 K_{S}$ and a $300 \mathrm{MeV}$ track cut $104673 \pm 1133$ $K_{S}$; this difference between the two cuts is about a third of the $K_{S}$. To reduce the charge asymmetry and move into a $p_{T}$ track range where the CTC functions more efficiently without losing too many $K_{S}$, a $300 \mathrm{MeV}$ track cut is selected. In top of Figure 3.4, the track cut is shown with the arrows as is the effect on the reconstructed $K_{S}$ mass spectra from the preceding cut [41] [43] [47] [48].

\section{The $\eta$ Cut}

The $K_{S}$ must have an $\eta$ less than 1.0. Beyond this $\eta$ range, the acceptance of the CTC is more limited, and hence, the track efficiency, along with the $K_{S}$ efficiency, drops significantly. The $\eta$ cut is displayed in the bottom of Figure 3.3 along with the mass distribution following the this cut [41] [43] [47] [48].

\section{The 3D Displacement Cut}

The 3D displacement is defined as the magnitude of the displacement between the secondary and the primary vertices. In particular, in the following equation:

$3 D$ Dis $=\sqrt{\left(v_{x}^{\text {secondary }}-v_{x}^{\text {primary }}\right)^{2}+\left(v_{y}^{\text {secondary }}-v_{y}^{\text {primary }}\right)^{2}+\left(v_{z}^{\text {secondary }}-v_{z}^{\text {primary }}\right)^{2}}$

where $v_{x}^{\text {primary }}, v_{y}^{\text {primary }}$, and $v_{z}^{\text {primary }}$ denote the location of the primary vertex, and $v_{x}^{\text {secondary }}, v_{y}^{\text {secondary }}$, and $v_{z}^{\text {secondary }}$ give the coordinates of the secondary vertex. Both vertices are measured with the respect to the origin of the detector. The $3 \mathrm{D}$ displacement of the $K_{S}$ candidates, $3 \mathrm{D}$ Dis, is must be greater than $1 \mathrm{~cm}$ to kept 
for further study. In the top of Figure 3.5, the 3D displacement is histogramed with the value of the cut being denoted by the arrows, and the effect of the cut on the reconstructed mass spectrum is also shown [41] [43] [47] [48].

\section{The Pointing Vector $\left(\operatorname{Cos}\left(\theta_{P D}\right)\right)$ Cut}

The pointing vector value is just the dot product of the $2 \mathrm{D}$ momentum vector and the $2 \mathrm{D}$ displacement divided by the product of the scalar values of the $2 \mathrm{D}$ momentum and 2D displacement. This value ensures that the momentum is along the displacement and is just the cosine of the angle between the 2D momentum and 2D displacement vectors. The pointing vector clusters around either -1 or 1 . If the value is around -1 , the candidate is traveling in the direction opposite to its displacement. In this case, the candidate is probably background. The closer the pointing vector value is to 1.0 , the better the candidate. In the bottom right of Figure 3.5 , the 2D pointing vector is plotted and beside the $K_{S}$ which pass a 0.990 pointing vector cut. The $2 \mathrm{D}$ version of this cut has better resolution than the $3 \mathrm{D}$ version because of the differences between the $z-r-\phi$ and $r-\phi$ resolution. Again, the momentum and secondary vertex values are calculated using the CTVMFT fitting routine, and the primary vertex is taken from the VTX [41] [45].

Below is a summary of the cuts used for $K_{S}$.

- $K_{S}$ Cuts

$-\chi_{K S}^{2}<20.0$

$-\left|z_{K S^{-}} z_{\text {primary }}\right|<3.0 \mathrm{~cm}$

- $p_{T}^{\text {tracks from } K S}>300 \mathrm{MeV}$

$-\left|\eta^{K S}\right|<1.0$

- 3D Displacement ${ }^{K S}>1.0 \mathrm{~cm}$ 
$-\cos \theta_{P D}^{K S}>0.990$ 

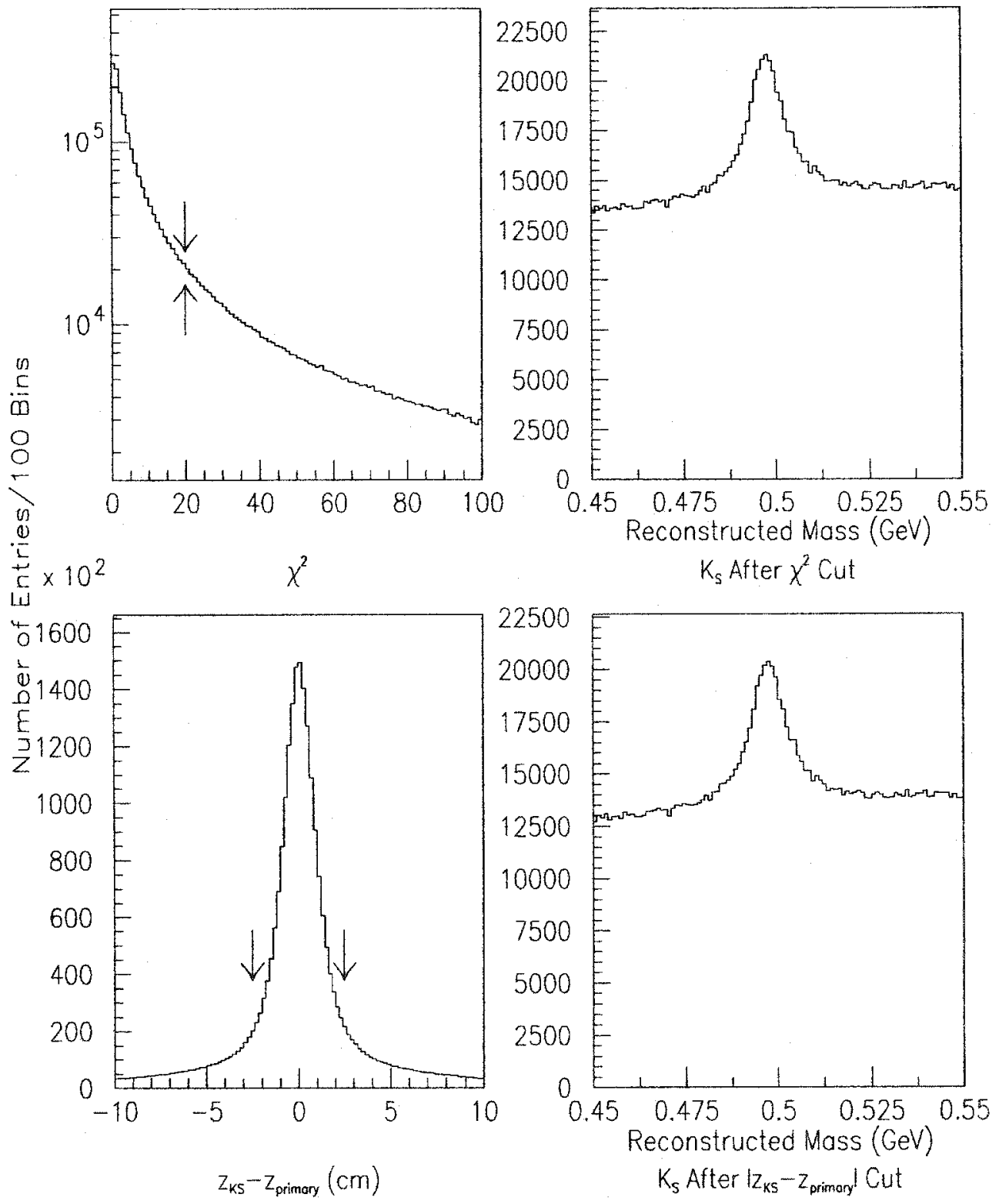

Figure 3.3: The $\chi^{2}$ distribution (upper left) and the $\left|z_{K S^{-}} z_{\text {primary }}\right|$ pointback distribution (lower left) are shown with arrows indicating the cut values. On the right side, the $K_{S}$ mass distribution after each cut. 

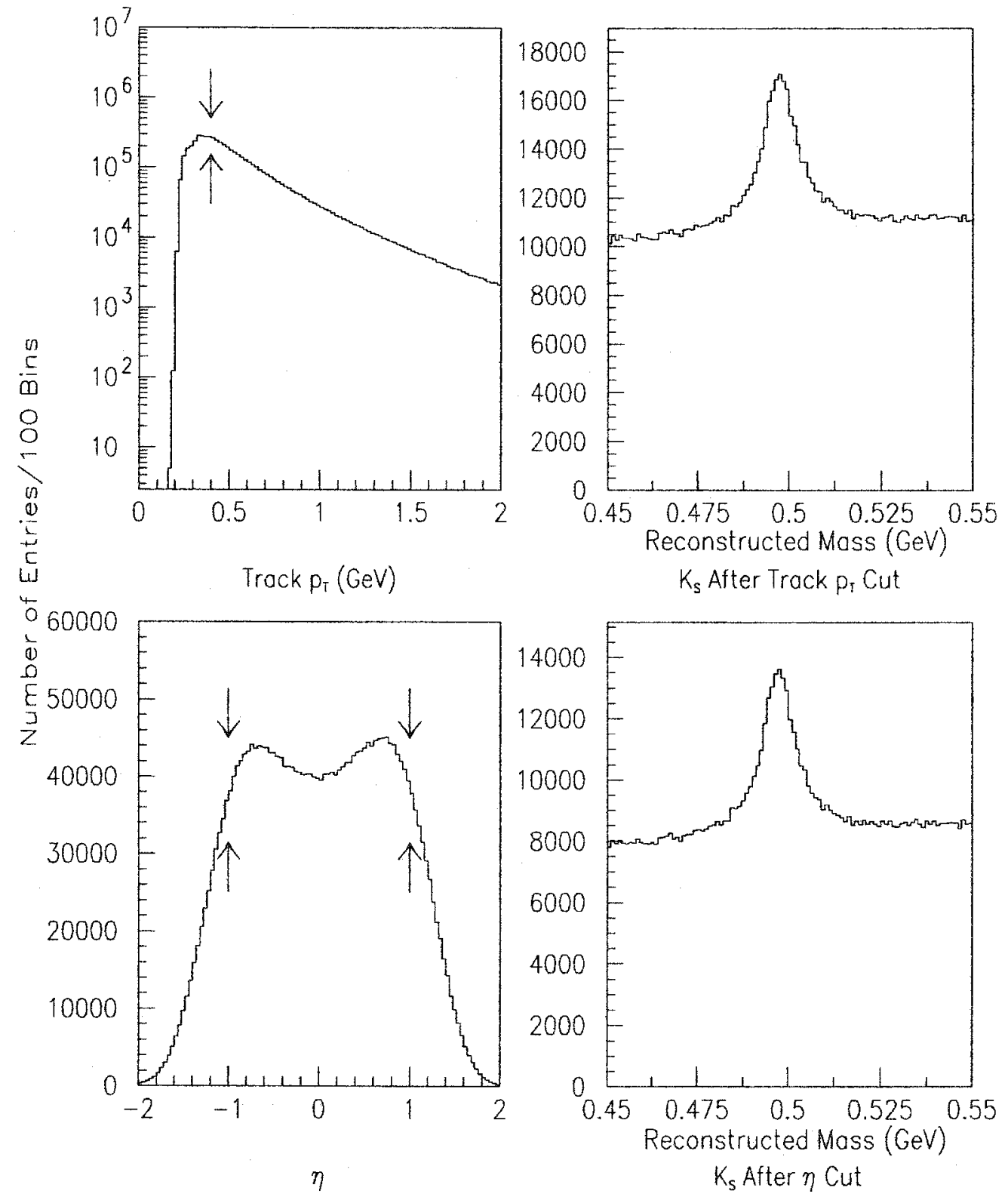

Figure 3.4: The track $p_{T}$ distribution (upper left) and the $\eta$ distribution (lower left) are shown with arrows indicating the cut values. On the right side, the $K_{S}$ mass distribution after each cut. 

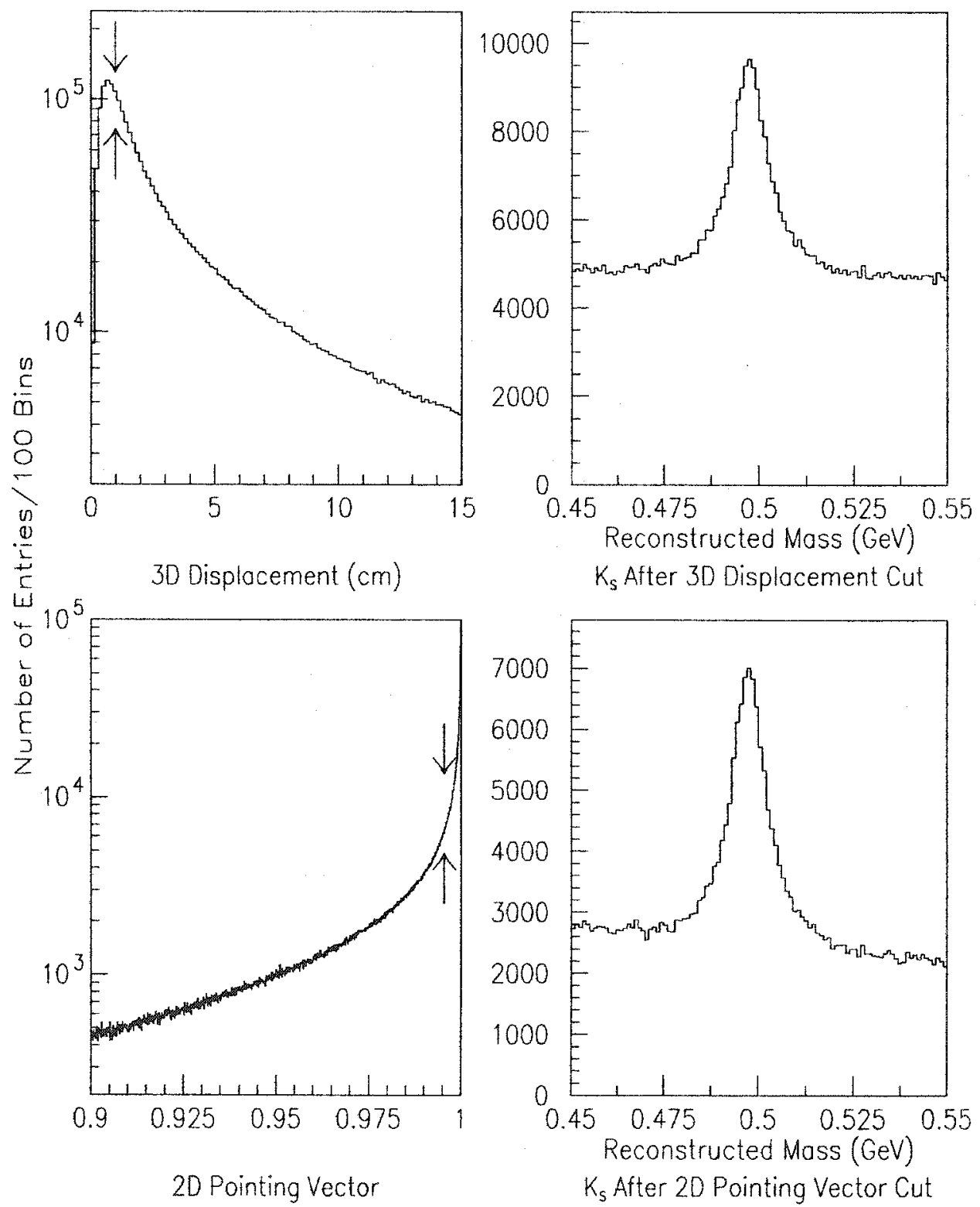

Figure 3.5: The 3D displacement distribution (upper left) and the $2 \mathrm{D}$ pointing vector distribution (lower left) are shown with arrows indicating the cut values. On the right side, the $K_{S}$ mass distribution after each cut. 


\section{Chapter 4}

\section{Minimum Bias: Track Embedding Efficiencies}

Not every particle produced in $p \bar{p}$ collisions is found by the CDF detector. So for every $K_{S}$ within a given $p_{T}$ range in the data, there are some that are missing. Hence, to correctly understand the physics, physics variables, such as the $p_{T}$ spectrum, must be corrected for the efficiency. Many are lost due a variety of reasons: holes in coverage, lost hits, extra hits causing reconstruction software to incorrectly reproduce tracks, secondary vertices not found, and the cuts to reduce background that also diminish the signal. Every explanation of an efficiency loss depends on the plethora of conditions such as the properties of the particles, the resolution, and the coverage of the detector. It is quite impractical to account for every loss in efficiency. Instead, one variable is selected to correct for the efficiency of the $K_{S}$, and all other factors for every property for every reason are integrated together to yield an overall correction factor for a given $p_{T}$. The $p_{T}$ variable is chosen because the CTC efficiency of finding a $K_{S}$ is strongly correlated to the $p_{T}$ of the individual daughter tracks from the parent particle which is itself is directly related to the $p_{T}$ of the $K_{S}$. Another efficiency correction on a variable, called the $c \tau$ and will be defined later, is computed in order to check the Monte Carlo. The main goal of this chapter is to describe how and why the efficiency curves are generated [41]. 


\subsection{An Overview}

The information obtained from creating Monte Carlo $K_{S}$ at a given $p_{T}$ is converted into hits in the CTC; and, after rerunning reconstruction software, the $K_{S}$ efficiency in data is determined for a given $p_{T}$. Once $K_{S} \quad p_{T}$ curves have been extracted from data, this efficiency curve as a function of $p_{T}$ is employed to correct for the inefficiency of the CTC. In addition, with the identical track embedding data, $c \tau$ efficiency as function of $p_{T}$ will also be determined.

Before describing the exact procedure, it is nice to visualize the track embedding concept. In Figure 4.1, a transverse view of the CTC is given for an event selected for track embedding. The dots in the event display are the actual hits in the CTC, and the fitting of these hits yields the tracks given by solid lines. Also, the greater the $p_{T}$, the less visible the curvature of a track. In Figure 4.2, the event in Figure 4.1 has had $3.0 \mathrm{GeV} K_{S}$ embedded into it. The high $p_{T}$ pion track is the new straight track, and the low $p_{T}$ pion track is the new curved track. These new track embedded pion tracks intersect, forming a $\mathrm{V}$, at the same space point the $K_{S}$ decays. Note since only charged particles create hits in the CTC, only the charged pions from the embedded $K_{S}$ can be seen directly. Thus, the neutral $K_{S}$ are invisible to the CTC. However, some events in which track embedding is attempted does not successfully reveal a $K_{S}$ even indirectly. The $K_{S}$, if the $p_{T}$ is too large, can decay outside the CTC. Moreover, the $K_{S}$ can escape down the beamline or slip through a crack so that it is never found. The daughter particles also may escape the detection of CTC, especially if the $p_{T}$ of the individual tracks is too soft. This is quite often the case with pions and explains most of the loss of efficiency for $K_{S}$. The efficiency is given as the number of $K_{S}$ found divided by the number of the events that $K_{S}$ were track embedded into, and the next section outlines the procedure [41]. 


\subsection{The Track Embedding Procedure}

\section{Step 1) Create text files of 4-vector and vertice data of $K_{S}$ daughters.}

Since $K_{S}$ are assumed not to prefer any particular direction in either $\eta(|\eta|<2.0)$ or $\phi(0 \leq \phi<2 \pi), K_{S}$ are generated one particle at a time at a specific $p_{T}$ at random $\phi(0 \leq \phi<2 \pi)$ and random $\eta(|\eta|<2.0)$ using a generator called FAKEEVENT [49]. Only $K_{S}$ are present after the generator level, and $K_{S}$ are actually decayed into charged pions in the CDFSIM detector simulator [50]. This is the case because it is possible that either the $K_{S}$ may live long enough to decay in a region beyond the CTC or its daughters have low enough $p_{T}$ so that the magnetic field of the solenoid might have a considerable effect on the tracks. Using the 4-vectors of the $K_{S}$ from FAKEEVENT as input, the CDFSIM detector simulator determines where the particle decays inside the detector as well as calculates the 4-vectors of the daughters and the decay vertices. After both the FAKEEVENT generator and the CDFSIM detector simulator, a list of daughter 4-vectors, primary vertices, and secondary vertices are placed into a text file for $K_{S}$ with a particular $p_{T}$. It is this information that will be ultimately be converted into CTC hits in a real data event [51].

\section{Step 2) Select a Minimum Bias data embedding sample.}

About 8,000 to 16,000 actual Minimum Bias events are needed for the track embedding study. The Minimum Bias events just pass the Minimum Bias triggers of the CDF detector, and hence each Minimum Bias event usually has a lower track multiplicity and fewer CTC hits when compared with higher $p_{T}$ events such as jet events. The Minimum Bias sample comprising of the data described in Chapter 3 will not suffice because CTC hit information is dropped prior to the creation of this data set. Instead, the Minimum Bias sample actually taken for this Minimum Bias 
track embedding analysis is the parent set of the Minimum Bias data in Chapter 3 , before the hit information is lost. The track embedding sample is also checked to have a luminosity distribution similar to the actual data as well as pass all the criteria specified for a good Minimum Bias event. The event must pass quality event cuts outlined in Chapter 3, and a tally of good track embedding events is $\operatorname{kept}[41][51]$.

\section{Step 3) Embed $K_{S}$ in Minimum Bias data.}

For each data event, the daughter information from a Monte Carlo generated and detector simulated $K_{S}$ at a given $p_{T}$ is taken from the text file. First, both the primary and secondary vertices are translated so the primary vertices of the data and Monte Carlo event coincide. Then, the 4-vectors of the daughter particles are converted into track parameters using a routine called QTRKPR [52]. From these output track parameters, hits are then added to the CTC taking into account multiple scattering, $\frac{d E}{d x}$ effects, and wire hit efficiencies using another CDF program called CTADDH [53] developed for this purpose. Once hits are added to the data, reconstruction is rerun to produce a new list of tracks which code can then be applied to discover whether $K_{S}$ can be found [28] [42] [51] [54] [55] [56] [57] [58].

\section{Step 4) Finding the $K_{S}$ in the track-embedded data.}

The technique implemented in the search for $K_{S}$ in the track embedding sample is for the most part identical to that outlined in Chapter 3 with the exception of employing a reconstructed $K_{S} \quad p_{T}$ window cut. Since the $p_{T}$ of track embedded particle is known, the information can be utilized to separate the embedded $K_{S}$ from $K_{S}$ already present in the data set. Since CTC resolution is generally a decreasing function of $p_{T}$ and would give rise to a broadening of the $p_{T}$ distribution as $p_{T}$ of the $K_{S}$ increases, the $p_{T}$ window must become larger as the $p_{T}$ of $K_{S}$ 
increases. The $p_{T}$ windows applied to each $K_{S} \quad p_{T}$ range is given by Table 4.1.

In Figure 4.3 , the $p_{T}$ windows are clearly marked by arrows in $K_{S} \quad p_{T}$ spectra produced after track embedding. Just as in the case with the reconstructed masses, $0.7 \mathrm{GeV}, 1.6 \mathrm{GeV}, 3.0 \mathrm{GeV}$, and $10.0 \mathrm{GeV}$ embedded $K_{S}$ with a $50 \mathrm{MeV}, 100 \mathrm{MeV}$, $250 \mathrm{MeV}$, and $1.0 \mathrm{GeV} p_{T}$ window cut values about the embedded $p_{T}$ values are shown. Each peak in the $p_{T}$ spectra represents the track embedded $K_{S}$, and its value shifts depending upon the embedded $p_{T}$ value. Similarly, the resolution is directly proportional to the embedded $p_{T}$. In fact, in Figure 4.4, the $p_{T}$ resolution increases from approximately $0.0045 \mathrm{GeV}$ for $0.7 \mathrm{GeV}$ track embedded $K_{S}$ to about $0.1748 \mathrm{GeV}$ for $10.0 \mathrm{GeV}$ track embedded $K_{S}$. Again, the $p_{T}$ windows should be wide enough to accept the embedded $K_{S}$ as well as be sufficiently narrow in order to reduce the number of candidates already in the data set for being mistaken for those being embedded. It should be noted that the track embedded $K_{S}$ resolutions are slightly narrower than the resolutions found in the Minimum Bias data.

Prior to $p_{T}$ window cuts, the reconstructed masses of $K_{S}$ candidates are shown in Figure 4.5. To help illustrate the effectiveness of the $p_{T}$ window cut, in Figure 4.6, the reconstructed masses of $K_{S}$ candidates are plotted for $K_{S}$ that pass (dashed line) and fail (solid line) the $p_{T}$ window cut. In this plot, $0.7 \mathrm{GeV}, 1.6 \mathrm{GeV}, 3.0 \mathrm{GeV}$, and $10.0 \mathrm{GeV}$ embedded $K_{S}$ with a $50 \mathrm{MeV}, 100 \mathrm{MeV}, 250 \mathrm{MeV}$, and $1.0 \mathrm{GeV} p_{T}$ window cuts about the embedded $p_{T}$ values are displayed. The peak in the solid line distribution are the $K_{S}$ already present in the data prior to track embedding. Those $K_{S}$ candidates that meet the $p_{T}$ window criteria are fitted simultaneously with a 1st order polynomial and a Gaussian. Then, the 1st order polynomial, basically the fit of the background, is needed in order to subtract out the background from the mass plots which pass the $p_{T}$ window cuts. The resulting fitted plots of the mass 
distributions with the background subtracted out from them are shown in Figure 4.7. The resolution of the $K_{S}$ approximately doubles from about $0.005 \mathrm{GeV}$ for the $0.7 \mathrm{GeV}$ case to approximately $0.010 \mathrm{GeV}$ for the $10.0 \mathrm{GeV}$ track embedded $K_{S}$.

The other $K_{S}$ cuts along with the $p_{T}$ window cut used in the generation of the $p_{T}$ efficiency curve are given below:

- $K_{S}$ in $p_{T}$ efficiency study

- Event Cuts

- $K_{S}$ must pass the $p_{T}$ window cut given by Table 4.1 .

$-\chi_{K S}^{2}<20.0$

$-\left|z_{K S}-z_{\text {primary }}\right|<3.0 \mathrm{~cm}$

- $p_{T}^{\text {tracks from } K S}>300 \mathrm{MeV}$

$-\left|\eta^{K S}\right|<1.0$

- 3D Displacement ${ }^{K S}>1.0 \mathrm{~cm}$

$-\cos \theta_{P D}^{K S}>0.990$

In Figures 4.8 through 4.10, the various cut values are shown for both the $K_{S}$ candidates within a given $p_{T}$ window (dashed line) and those $K_{S}$ candidates which lie outside the $p_{T}$ window (solid line). The solid line can be for the most part be considered as the background distribution whereas the dashed line contains mostly track embedded $K_{S}$ candidates. The arrows indicate the cut values for each variable specified in the itemized list above. Moreover, this is done for both $1.6 \mathrm{GeV}$ and $3.0 \mathrm{GeV}$ track embedded $K_{S}$ with $p_{T}$ window cuts of $100 \mathrm{MeV}$ and $250 \mathrm{MeV}$ respectively. To be more specific, Figure 4.8 shows the $\chi_{K S}^{2}$ and $z_{K S^{-}} z_{\text {primary }}$ distributions, Figure 4.9 displays the $p_{T}^{\text {tracks from } K S}$ and $\eta^{K S}$ distributions, and Figure 
4.10 histograms 3D Displacement ${ }^{K S}$ and $\cos \theta_{P D}^{K S}$ distributions. Furthermore, since the number of background is far greater than the track embedded $K_{S}$, these distributions are normalize to unity.

In Tables 4.2 through 4.4 , the effect of each cut on the track embedded Monte Carlo is compared with the data for a $p_{T}$ range that is approximately around the track embedded $p_{T}$ value. The motivation of these ranges in the data is that there lacks sufficient statistics to compute $K_{S}$ within extremely narrow $p_{T}$ windows. As a result, $K_{S}$ from the $0.7 \mathrm{GeV}$ track embedded Monte Carlo are matched with $K_{S}$ with $p_{T}$ between 650 and $750 \mathrm{GeV}$ in the data. Likewise, $1.6 \mathrm{GeV}$ track embedded $K_{S}$ are equated with $1.5-1.7 \mathrm{GeV} K_{S}$ in data, and the $3.0 \mathrm{GeV}$ track embedded $K_{S}$ are set against the $2.75-3.25 \mathrm{GeV} K_{S}$ in data. The percentage drops in Tables 4.2 through 4.4 are measured with respect to the $\chi_{K S}^{2}$ in order to demand $K_{S}$ in the data to originate from the primary just like the $K_{S}$ in track embedding sample. There is a very small effect of the $\chi_{K S}^{2}$ cut on the track embedded $K_{S}$, but the $\chi_{K S}^{2}$ cut effects the data by $20-40 \%$. This is believed to be due to $K_{S}$ in the data not originating from the leading primary vertex. These $K_{S}$ that would fail the pointing criteria, and subsequently, the $\chi_{K S}^{2}$ cut. However, the agreement after the $\chi_{K S}^{2}$ is good to a few percent with the exception of the $0.7 \mathrm{GeV}$ case of the $\cos \theta_{P D}^{K S}$ cut where the difference is on the order of $15 \%$.

Step 5) Calculate efficiencies with the new track-embedded data set. For every track embedded $p_{T}$ value, the number of $K_{S}$ found after cuts is divided by the number of track embedded events, and this process repeats for a multitude of different $p_{T}$ values between $0.5 \mathrm{GeV}$ and $10.0 \mathrm{GeV}$. In particular, the number of $K_{S}$ after cuts is computed by summing $K_{S}$ candidates between 0.48 and 0.52 $\mathrm{GeV}$ after the background has been subtracted out. For cases where the efficiencies 
Table 4.1: The $p_{T}$ window ranges for a given $K_{S} p_{T}$.

\begin{tabular}{|c|c|}
\hline$p_{T}$ Range of $K_{S}(\mathrm{GeV})$ & $p_{T}$ Window Range $(\mathrm{MeV})$ \\
\hline$p_{T} \leq 1.4$ & 50.0 \\
$1.4<p_{T} \leq 1.8$ & 100.0 \\
$1.8<p_{T} \leq 2.3$ & 150.0 \\
$2.3<p_{T} \leq 3.0$ & 250.0 \\
$3.0<p_{T} \leq 5.0$ & 500.0 \\
$5.0<p_{T} \leq 10.0$ & 1000.0 \\
\hline
\end{tabular}

Table 4.2: The number of $K_{S}$ after each successive cut for $p_{T}=0.7 \mathrm{GeV}$. Errors are taken from the fit of the area of the Gaussian. There are $16677 K_{S}$ embedded in $|\eta|<2.0$, and events pass all cuts.

\begin{tabular}{|c|c|c|c|}
\hline Cuts & $\begin{array}{c}\text { Number of } K_{S} \\
\text { in MC }\end{array}$ & $\%$ & $\begin{array}{c}\% \text { in Data } \\
(.65-.75 \mathrm{GeV})\end{array}$ \\
\hline After Event Cuts & $2186 . \pm 61$ & $1.015 \pm 0.0384$ & $1.421 \pm 0.0384$ \\
\hline$\chi_{K S}^{2}<20.0$ & $2153 . \pm 55$ & 1.000 & 1.000 \\
\hline$\left|z_{K S}-z_{\text {primary }}\right|<3.0 \mathrm{~cm}$ & $2092 . \pm 54$ & $0.972 \pm 0.0353$ & $0.943 \pm 0.0353$ \\
\hline$p_{T}^{\text {tracks from } K S}>300 \mathrm{MeV}$ & 1556. \pm 46 & $0.723 \pm 0.0282$ & $0.740 \pm 0.0282$ \\
\hline$\left|\eta^{K S}\right|<1.0$ & 1261. 土40. & $0.586 \pm 0.0239$ & $0.576 \pm 0.0239$ \\
\hline 3D Displacement ${ }^{K S}>1.0 \mathrm{~cm}$ & $1075 . \pm 36$ & $0.499 \pm 0.0210$ & $0.507 \pm 0.0210$ \\
\hline $\cos \theta_{P D}^{K S}>0.990$ & 998. \pm 33 . & $0.464 \pm 0.0194$ & $0.412 \pm 0.0194$ \\
\hline
\end{tabular}

are low (i.e. $p_{T}$ below $1.0 \mathrm{GeV}$ ), about 16,000 Minimum Bias events are needed to calculate the efficiency for those $p_{T}$ intervals. Then, every computed efficiency point is plotted against $p_{T}$ with errors from the fit in order to produce plots in Figures 4.11. In Figure 4.11, the upper portion consists of each efficiency point as a function of $p_{T}$. The lower left plot has the front end (below $4 \mathrm{GeV}$ ) fitted with a 6 th order polynomial, and the lower right plot has the back end (above $4 \mathrm{GeV}$ ) fitted with a 1st order polynomial. These fitted curves are later taken to correct the background subtracted $p_{T}$ distribution of the $K_{S}$ in the data. 
Table 4.3: The number of $K_{S}$ after each successive cut for $p_{T}=1.6 \mathrm{GeV}$. The errors are taken from the fit of the area of the Gaussian. There are $8028 K_{S}$ embedded in $|\eta|<2.0$, and events pass all cuts.

\begin{tabular}{|c|c|c|c|}
\hline Cuts & $\begin{array}{c}\text { Number of } K_{S} \\
\text { in MC }\end{array}$ & $\%$ & $\begin{array}{c}\text { \% in Data } \\
(1.5-1.7 \mathrm{GeV})\end{array}$ \\
\hline $\begin{array}{l}\text { After Event Cuts } \\
\gamma^{2}<200\end{array}$ & $\begin{array}{l}3222 . \pm 62 . \\
3141+60 .\end{array}$ & $\begin{array}{c}1.026 \pm 0.0278 \\
1.000\end{array}$ & $\begin{array}{c}1.174 \pm 0.0278 \\
1.000\end{array}$ \\
\hline $\begin{array}{c}\chi_{K S}<20.0 \\
K S-z_{\text {mrimaru }}<3.0 \mathrm{~cm}\end{array}$ & 3109. \pm 59 & $0.990 \pm 0.0267$ & $0.972 \pm 0.0267$ \\
\hline$p_{T}^{\text {tracks from } K S}>300 \mathrm{MeV}$ & 2961. 土58. & $0.943 \pm 0.0258$ & $0.944 \pm 0.0258$ \\
\hline$\left|\eta^{K S}\right|<1.0$ & 2397. \pm 51 & $0.763 \pm 0.0218$ & $0.775 \pm 0.0218$ \\
\hline $3 \mathrm{D}$ Displacement ${ }^{K S}>1.0 \mathrm{~cm}$ & $2219 . \pm 49$. & $0.706 \pm 0.0206$ & $0.736 \pm 0.0206$ \\
\hline $\cos \theta_{P D}^{K S}>0.990$ & 2221. 土48. & $0.707 \pm 0.0204$ & $0.715 \pm 0.0204$ \\
\hline
\end{tabular}

Table 4.4: The number of $K_{S}$ after each successive cut for $p_{T}=3.0 \mathrm{GeV}$. The errors are taken from the fit of the area of the Gaussian. There are $8028 K_{S}$ embedded in $|\eta|<2.0$, and events pass all cuts.

\begin{tabular}{|c|c|c|c|}
\hline Cuts & $\begin{array}{c}\text { Number of } K_{S} \\
\text { in MC }\end{array}$ & $\%$ & $\begin{array}{c}\% \text { in Data } \\
(2.75-3.25 \mathrm{GeV})\end{array}$ \\
\hline After Event Cuts & $3978 . \pm 68$ & $1.011 \pm 0.0242$ & $1.199 \pm 0.0242$ \\
\hline$\chi_{K S}^{2}<20.0$ & 3937. \pm 66 . & 1.000 & 1.000 \\
\hline$\left|z_{K S}-z_{\text {primary }}\right|<3.0 \mathrm{~cm}$ & $3912 . \pm 67$. & $0.994 \pm 0.0238$ & $0.979 \pm 0.0238$ \\
\hline$p_{T}^{\text {tracks from } K S}>300 \mathrm{MeV}$ & 3831. \pm 66 . & $0.973 \pm 0.0234$ & $0.968 \pm 0.0234$ \\
\hline$\left|\eta^{K S}\right|<1.0$ & $3131 . \pm 58$. & $0.795 \pm 0.0198$ & $0.823 \pm 0.0199$ \\
\hline $3 \mathrm{D}$ Displacement $^{K S}>1.0 \mathrm{~cm}$ & $3008 . \pm 57$ & $0.764 \pm 0.0193$ & $0.768 \pm 0.0193$ \\
\hline $\cos \theta_{P D}^{K S}>0.990$ & $3005 . \pm 57$ & $0.763 \pm 0.0193$ & $0.748 \pm 0.0193$ \\
\hline
\end{tabular}




\subsection{The c $\tau$ Corrections}

One efficiency correction that will not appear again when studying jet events is that of the proper lifetime, $\mathrm{c} \tau$, of $K_{S}$. The proper life time, $c \tau$, is given by the following equation:

$$
c \tau=\frac{M_{K S} D_{K S}}{P_{K S}}
$$

where $P_{K S}$ is the $3 \mathrm{D}$ momentum of the $K_{S}, D_{K S}$ is the $3 \mathrm{D}$ displacement of the $K_{S}$, and $M_{K S}$ is the mass of the $K_{S}$. Although $\mathrm{c} \tau$ is regarded as a positive quantity, a measurement error in any component in either $P_{K S}$ or $D_{K S}$ can give rise to negative values [40] [59] [60]. Since the statistics are typically much smaller for negative $\mathrm{ct}$ values, the positive $c \tau$ values are studied. These $c \tau$ efficiency corrections will be incorporated into the $\mathrm{c} \tau$ measurements in Chapter 5 . The goal is not to measure the c $\tau$ itself but instead to check the accuracy of the efficiency corrections. The more precise the resulting efficiency-corrected $c \tau$, the better the efficiency curve actually is.

The experimental check is not actually the $c \tau$ distribution itself but rather the negative of the reciprocal of the slope of the decaying exponential of the $c \tau$ distribution. For $K_{S}$, the accepted value is about $2.6786 \pm 0.0024 \mathrm{~cm}$ [9]. Although the $c \tau$ of particle depends upon the weighted average of each decay channel, the experimental value is sufficient for comparison since most decay channels have the same $\mathrm{c} \tau$. It is important to note that $K_{S}$ decaying from other particles, such as $B^{0} \rightarrow K_{S}+J / \Psi$ or $D_{0} \rightarrow K_{S}+$ anything, will have a $c \tau$ that will be slightly larger than the prompt experimental value. However, $B^{0}$ and $D_{0}$ have very small c $\tau$ themselves [9] [61], so the difference is negligible.

The $c \tau$ distributions of $K_{S}$ at specific $p_{T}$ values are produced with the following 
$K_{S}$ cuts:

- Sample $K_{S}$ in $c \tau$ Study

- Event Cuts

- $K_{S}$ must pass the $p_{T}$ window cut given by Table 4.1.

$-\chi_{K S}^{2}<20.0$

$-\left|z_{K S^{-}} z_{\text {primary }}\right|<3.0 \mathrm{~cm}$

- $p_{r}^{\text {tracks from } K S}>300 \mathrm{MeV}$

$-\left|\eta^{K S}\right|<1.0$

- 3D Displacement ${ }^{K S}>1.0 \mathrm{~cm}$

These cuts are looser than those taken to calculate the $K_{S} p_{T}$ efficiencies since some cuts are more likely to sculpt the $\mathrm{c} \tau$ spectrum than others. Moreover, applying all cuts may not yield enough statistics at certain $p_{T}$ values in order to get an accurate enough measurement of the absolute value of the slope of the $c \tau$. Regardless of cuts, the efficiency curves once applied to the data would yield approximately the same result with the exception of mild discontinuities in the final efficiency-corrected $\mathrm{c} \tau$ plot. The cuts incorporated here do not bias the $\mathrm{c} \tau$ measurement beyond 1.0 $\mathrm{cm}$, and in the end, the slope of the final $\mathrm{c} \tau$ spectrum in Chapter 5 is relatively smooth without dips or bumps above a $\mathrm{c} \tau$ of $1.0 \mathrm{~cm}$.

A subtle problem will now be described. After the $p_{T}$ window cut, the $c \tau$ can be plotted for $K_{S}$ masses between 0.48 and $0.52 \mathrm{GeV}$ (central region). Similarly, the c $\tau$ distributions can also be plotted from 0.45 to $0.48 \mathrm{GeV}$ as well as 0.52 to 0.55 $\mathrm{GeV}$ (sideband regions). Ideally, the $\mathrm{c} \tau$ distributions would be null histograms in sideband regions; however, they are not. Instead, there exists background $\mathrm{c} \tau$ values 
that appear in the sidebands. This is not the concern. The issue is that there are such background $\mathrm{c} \tau$ in the central region as well. Hence, when the $\mathrm{c} \tau$ of this central region is plotted, it includes the $\mathrm{c} \tau$ of the $K_{S}$ plus a very unwanted fraction of $\mathrm{c} \tau$ from the background candidates. Background subtraction is a process employed in order to remove unwanted background $\mathrm{c} \tau$ from the central mass region.

Background subtraction is process outlined as follows. First, compute the total number of $K_{S}$ in both of the sidebands $\left(N_{s 1}^{\text {total }}, N_{s 2}^{\text {total }}\right)$, the central region $\left(N_{c}^{\text {total }}\right)$, and the total number of $K_{S}$ in the central region after subtracting the background $\left(N_{K S}\right)$. Second, calculate the fraction of the background within the central peak as follows:

$$
\text { fraction }=\frac{N_{c}^{\text {total }}-N_{K S}^{\text {total }}}{N_{s 1}^{\text {total }}+N_{s 2}^{\text {total }}}
$$

Then, subtract from the $\mathrm{c} \tau$ of the central region the sum of the $\mathrm{c} \tau$ distributions of the sidebands by the fraction calculated from equation 4.2. Similarly, propagate the errors bin by bin with this fraction accordingly:

$$
\text { error }=\sqrt{N_{c}^{b i n}+\text { fraction } *\left(N_{s 1}^{b i n}+N_{s 2}^{b i n}\right)}
$$

where the number of particles in a given bin for the central region and the two sidebands are expressed as $N_{c}^{b i n}, N_{s 1}^{b i n}$, and $N_{s 2}^{b i n}$. After this background procedure, the resulting $\mathrm{c} \tau$ of the central region has had these background $\mathrm{c} \tau$ removed. The slopes from each $c \tau$ distributions at a given $p_{T}$ are plotted against the $p_{T}$ of the embedded $K_{S}$ in Figure 4.12.

Again, the slope of the $c \tau$ distribution is actually the negative reciprocal of the experimental measured $\mathrm{c} \tau$. For the implementation of these adjustments, it simpler to correct for the positive inverse of the experimental value, or in other words, just 
the absolute value of the slope of the $c \tau$ distribution. At large $c \tau$, particles decay deeper into the CTC such that the inner layers of the CTC have less hits. This explains the drop from the expectation value at high $\mathrm{c} \tau$ (or increase in the absolute value of the slope), see Figure 4.12. The high $\mathrm{c} \tau$ corrections effect the tail of the data distribution the most (high absolute value of the slope), where there are few $K_{S}$. In Chapter 5 , the efficiency-corrected $\mathrm{c} \tau$ will be computed from the data [62]. 


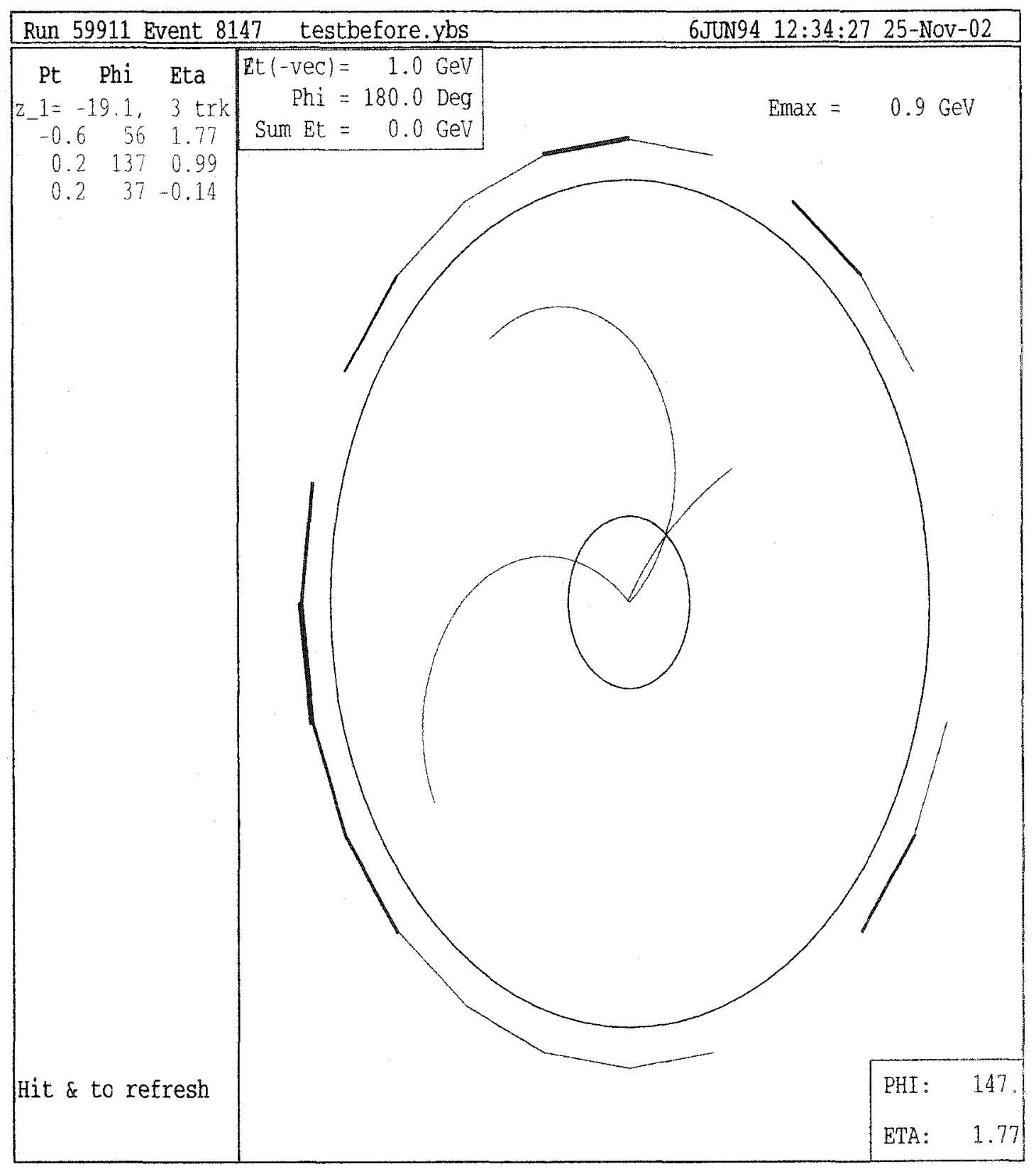

Figure 4.1: A transverse view of a Minimum Bias event. A $K_{S}$ has not yet been track embedded. 


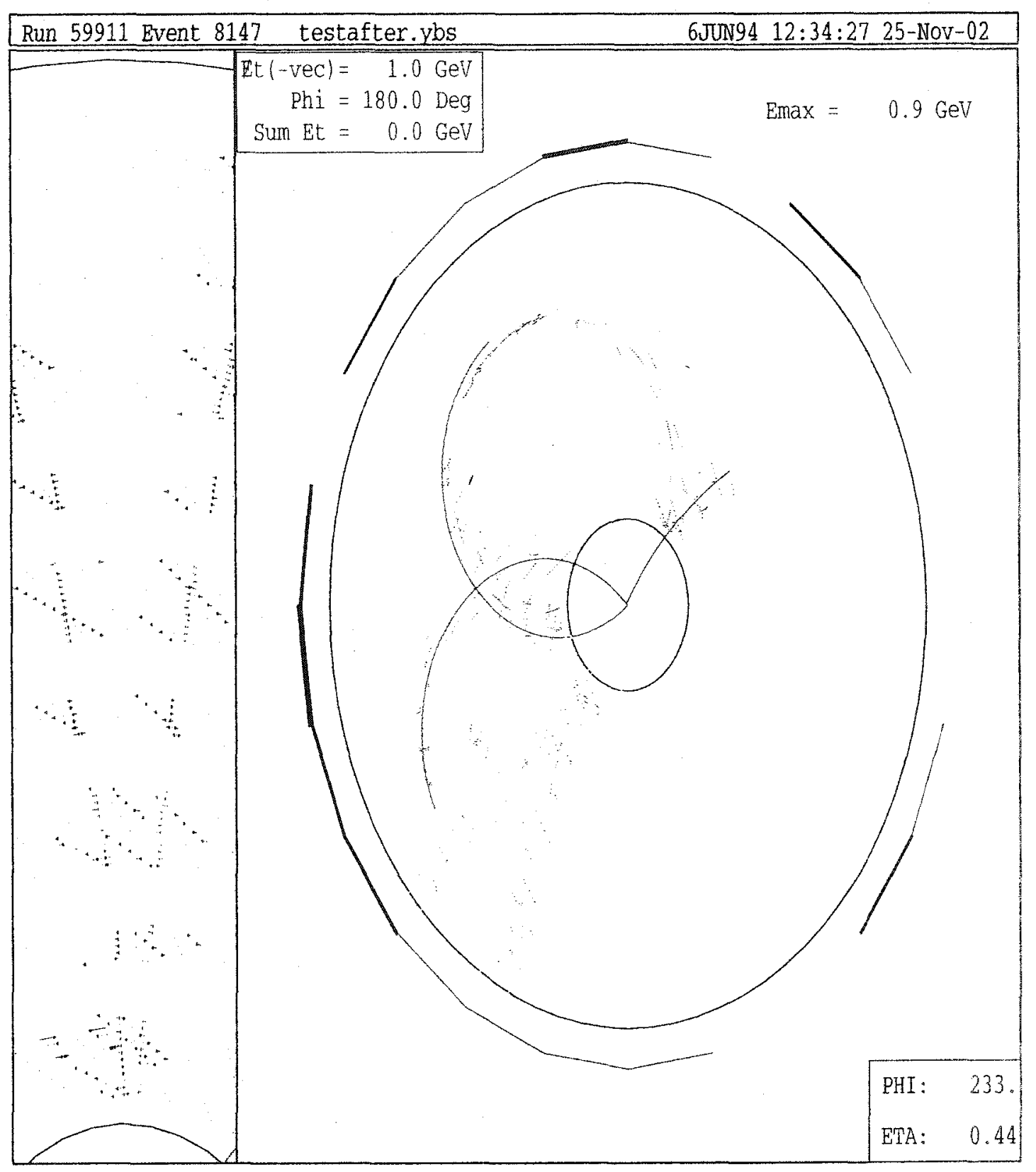

Figure 4.2: A transverse view of a Minimum Bias event with a $3.0 \mathrm{GeV}$ trackembedded $K_{S}$. The $K_{S}$ consists of two tracks intersecting to form a "V". This is not seen in Figure 4.1. The curved tracks have less transverse momenta than the tracks with less curvature. 

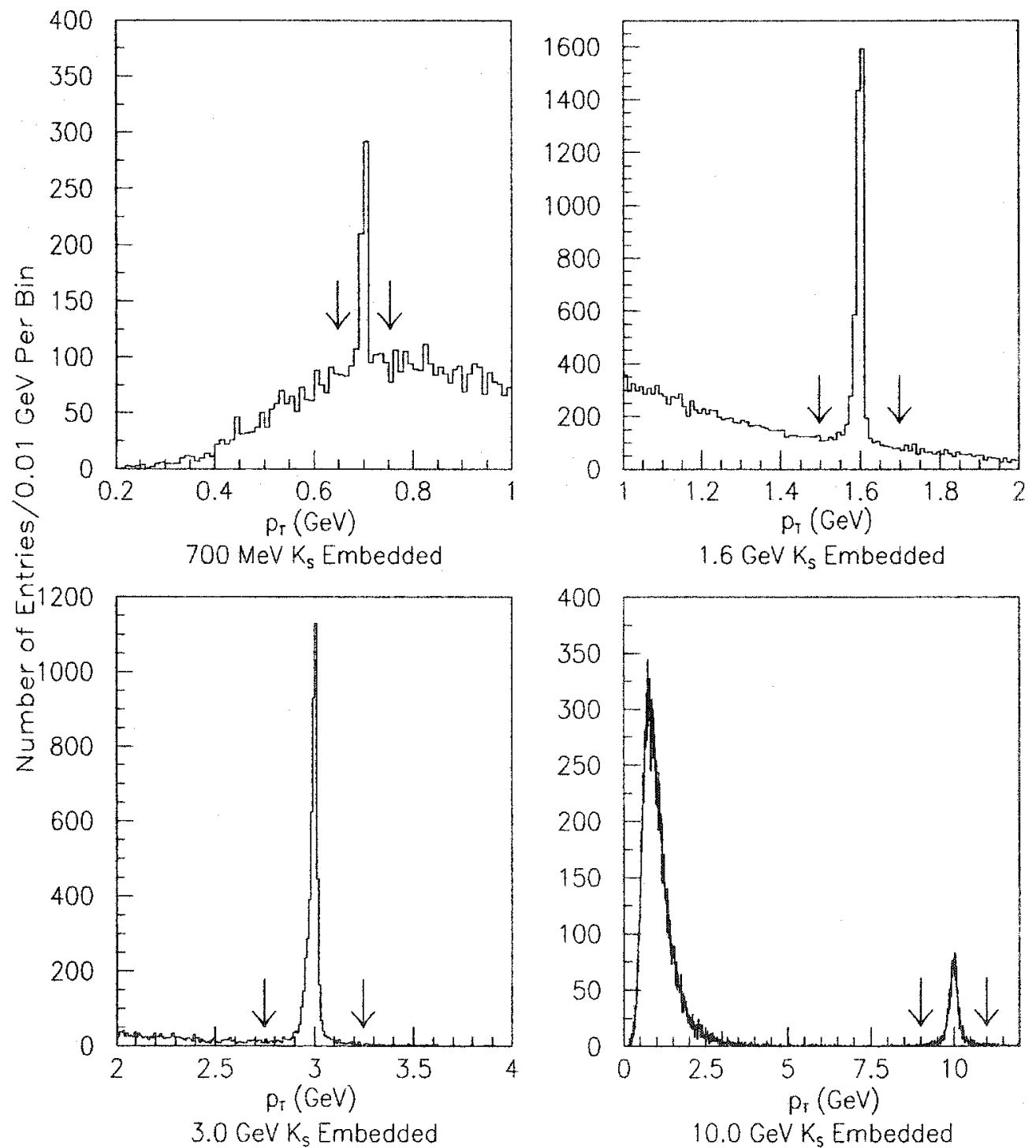

$1.6 \mathrm{GeV} \mathrm{K}_{\mathrm{s}}$ Embedded

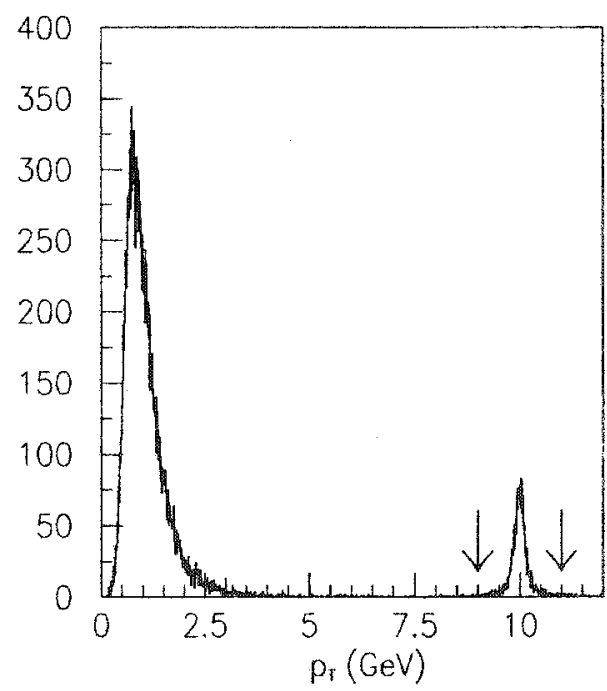

10.0 GeV K Embedded

Figure 4.3: The $p_{T}$ distribution of $K_{S}$ candidates after track embedding. The $p_{T}$ distributions of $0.7 \mathrm{GeV}, 1.6 \mathrm{GeV}, 3.0 \mathrm{GeV}$, and $10.0 \mathrm{GeV} K_{S}$ candidates with arrows indicating $p_{T}$ window cuts. The $p_{T}$ windows are $50 \mathrm{MeV}, 100 \mathrm{MeV}, 250$ $\mathrm{MeV}, 1.0 \mathrm{GeV}$ for $0.7 \mathrm{GeV}, 1.6 \mathrm{GeV}, 3.0 \mathrm{GeV}$, and $10.0 \mathrm{GeV} K_{S}$. These are based upon 10004 events for the $0.7 \mathrm{GeV}$ case and 8668 events for the $1.6 \mathrm{GeV}, 3.0 \mathrm{GeV}$, and $10.0 \mathrm{GeV}$ cases. These distributions are of $K_{S}$ embedded into $|\eta|<2.0$ prior to the event cuts. 

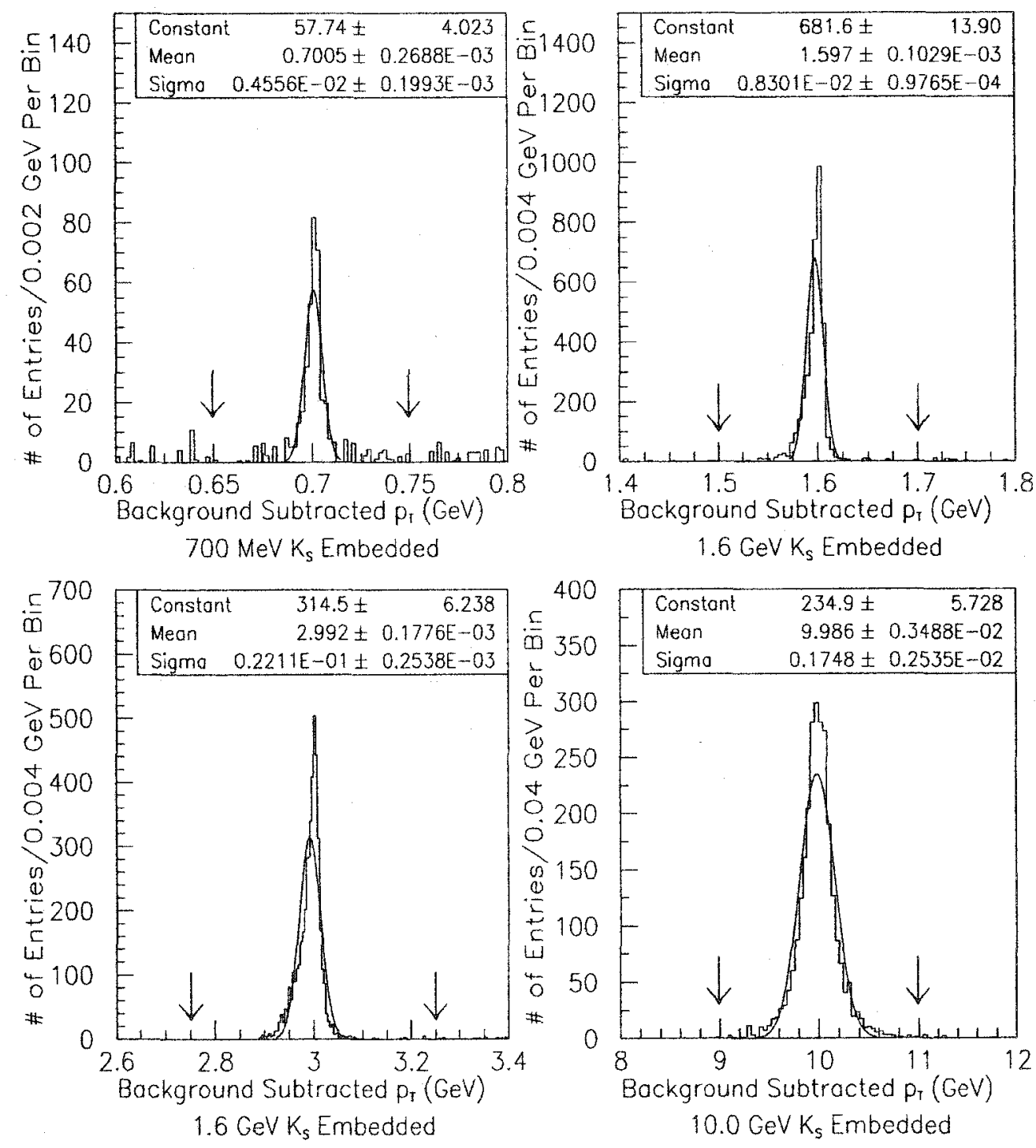

Figure 4.4: The $p_{T}$ distributions of $K_{S}$ candidates after background subtraction fitted with a gausian. The fitted background subtracted $p_{T}$ distributions of 0.7 $\mathrm{GeV}, 1.6 \mathrm{GeV}, 3.0 \mathrm{GeV}$, and $10.0 \mathrm{GeV} K_{S}$ candidates with the $p_{T}$ windows being $50 \mathrm{MeV}, 100 \mathrm{MeV}, 250 \mathrm{MeV}, 1.0 \mathrm{GeV}$ for $0.7 \mathrm{GeV}, 1.6 \mathrm{GeV}, 3.0 \mathrm{GeV}$, and 10.0 $\mathrm{GeV} K_{S}$. These are based upon 10004 events for the $0.7 \mathrm{GeV}$ case and 8668 events for the $1.6 \mathrm{GeV}, 3.0 \mathrm{GeV}$, and $10.0 \mathrm{GeV}$ cases. These distributions are of $K_{S}$ embedded into $|\eta|<2.0$ prior to the event cuts. 

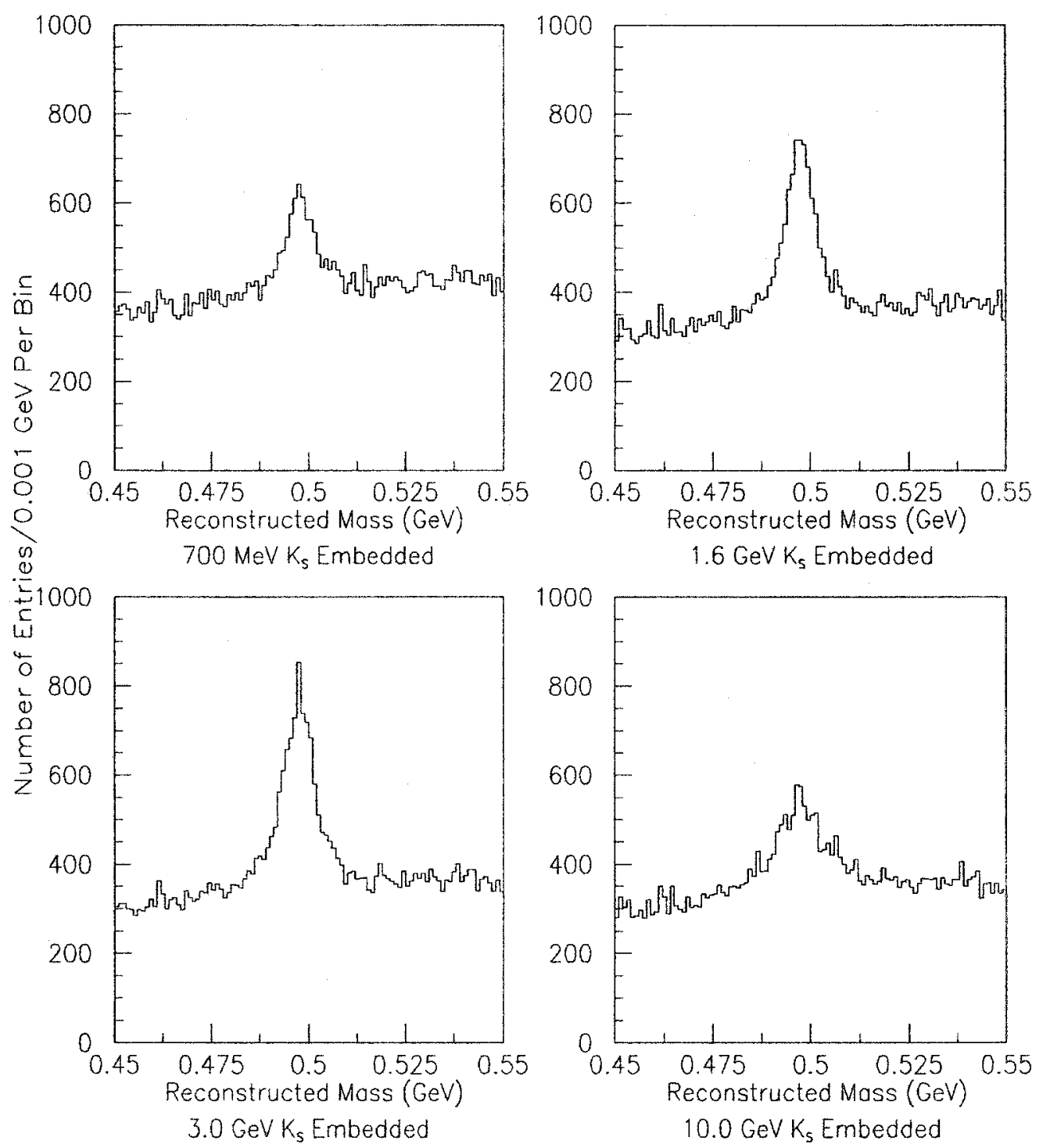

Figure 4.5: The mass distribution of $K_{S}$ candidates after track-embedding. The mass distributions of $0.7 \mathrm{GeV}, 1.6 \mathrm{GeV}, 3.0 \mathrm{GeV}$, and $10.0 \mathrm{GeV} K_{S}$ candidates that pass (dashed line) and fail (solid line) $p_{T}$ window. The $p_{T}$ windows are $50 \mathrm{MeV}$, $100 \mathrm{MeV}, 250 \mathrm{MeV}, 1.0 \mathrm{GeV}$ for $0.7 \mathrm{GeV}, 1.6 \mathrm{GeV}, 3.0 \mathrm{GeV}$, and $10.0 \mathrm{GeV} K_{S}$. These are based upon 10004 events for the $0.7 \mathrm{GeV}$ case and 8668 events for the $1.6 \mathrm{GeV}, 3.0 \mathrm{GeV}$, and $10.0 \mathrm{GeV}$ cases. These distributions are of $K_{S}$ embedded into $|\eta|<2.0$ prior to the event cuts. 

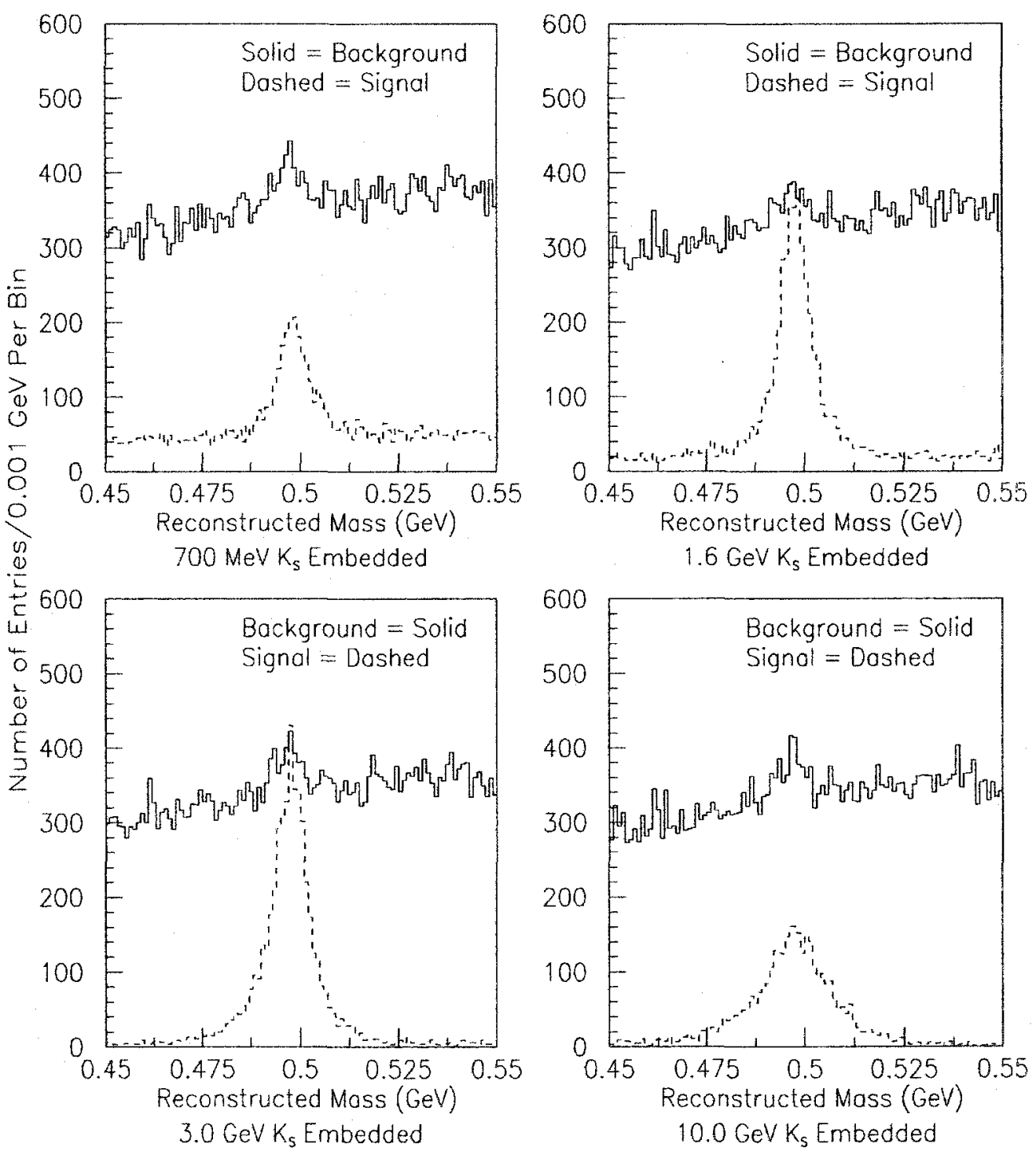

Figure 4.6: The mass distribution of $K_{S}$ candidates after track-embedding (with and without the $p_{T}$ window cut). The mass distributions of $0.7 \mathrm{GeV}, 1.6 \mathrm{GeV}, 3.0$ $\mathrm{GeV}$, and $10.0 \mathrm{GeV} K_{S}$ candidates that pass (dashed line) and fail (solid line) $p_{\mathrm{T}}$ window. The $p_{T}$ windows are $50 \mathrm{MeV}, 100 \mathrm{MeV}, 250 \mathrm{MeV}, 1.0 \mathrm{GeV}$ for $0.7 \mathrm{GeV}$, 1.6 GeV, 3.0 GeV, and 10.0 GeV $K_{S}$. These are based upon 10004 events for the $0.7 \mathrm{GeV}$ case and 8668 events for the $1.6 \mathrm{GeV}, 3.0 \mathrm{GeV}$, and $10.0 \mathrm{GeV}$ cases. These distributions are of $K_{S}$ embedded into $|\eta|<2.0$ prior to the event cuts. 

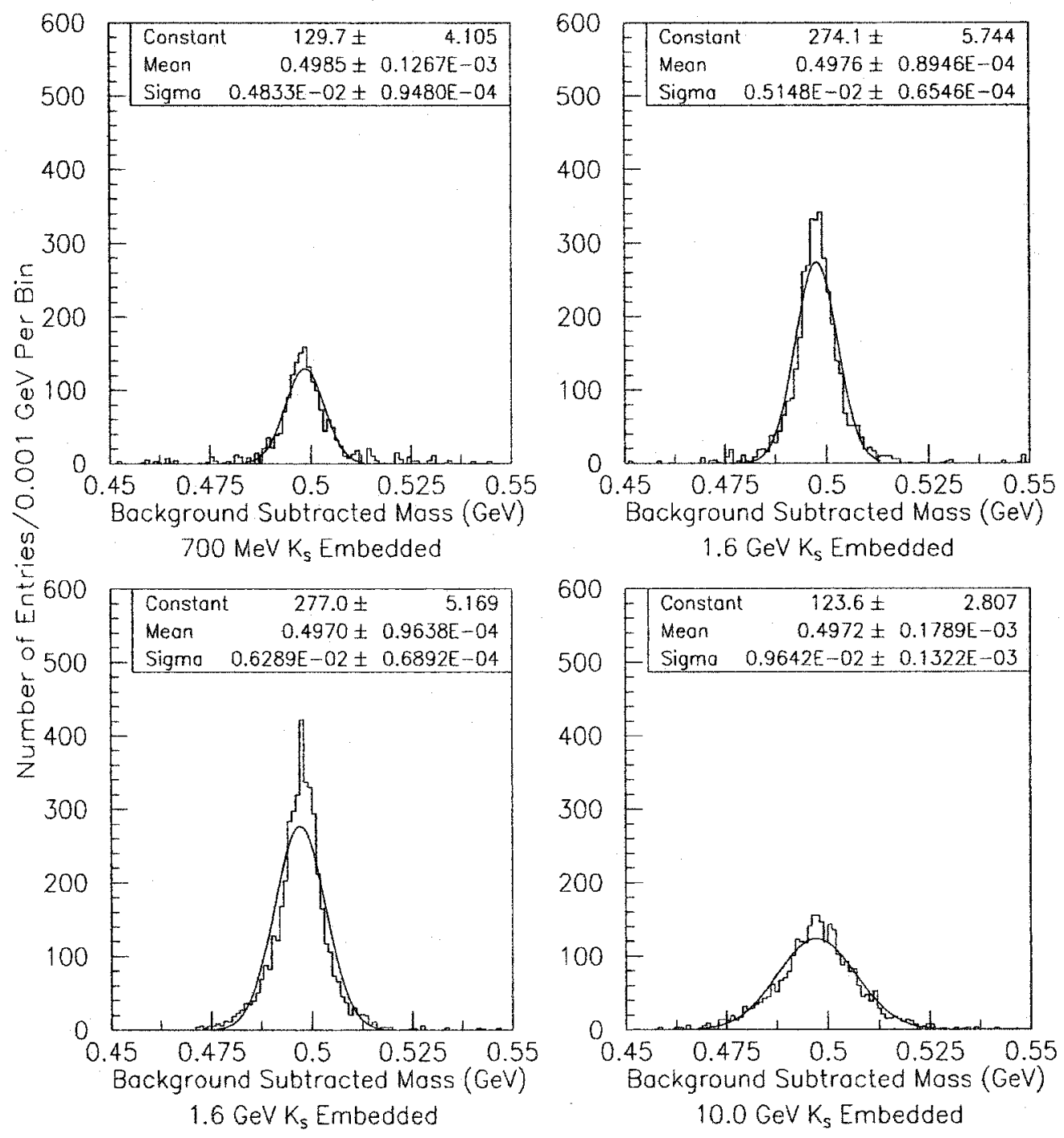

$1.6 \mathrm{GeV} K_{\mathrm{s}}$ Embedded

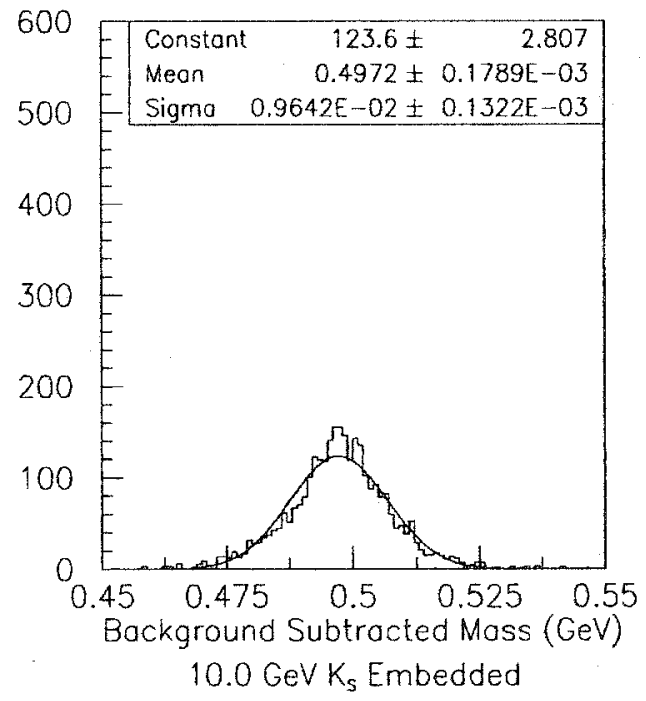

Figure 4.7: The mass distribution of $K_{S}$ candidates after background subtraction within the $p_{T}$ window. The fitted background subtracted mass distributions of 0.7 $\mathrm{GeV}, 1.6 \mathrm{GeV}, 3.0 \mathrm{GeV}$, and $10.0 \mathrm{GeV} K_{S}$ candidates that pass (dashed line) and fail (solid line) $p_{T}$ window. The $p_{T}$ windows are $50 \mathrm{MeV}, 100 \mathrm{MeV}, 250 \mathrm{MeV}, 1.0$ $\mathrm{GeV}$ for $0.7 \mathrm{GeV}, 1.6 \mathrm{GeV}, 3.0 \mathrm{GeV}$, and $10.0 \mathrm{GeV} K_{S}$. These are based upon 10004 events for the $0.7 \mathrm{GeV}$ case and 8668 events for the $1.6 \mathrm{GeV}, 3.0 \mathrm{GeV}$, and $10.0 \mathrm{GeV}$ cases. These distributions are of $K_{S}$ embedded into $|\eta|<2.0$ prior to the event cuts. 

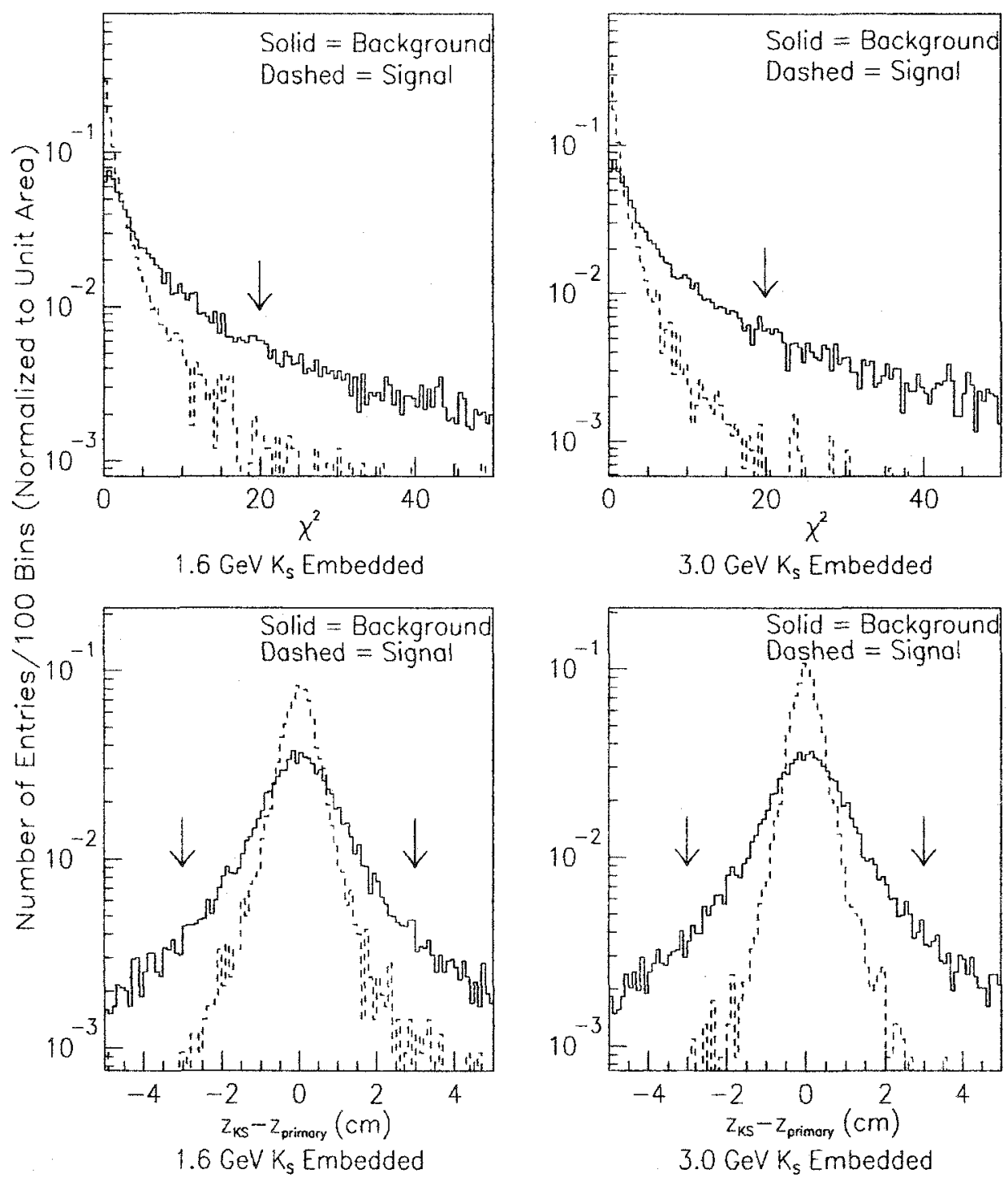

Figure 4.8: In the top half, the $\chi_{K S}^{2}$ distributions of the $1.6 \mathrm{GeV}$ (left) and 3.0 $\mathrm{GeV}$ (right) candidates which pass (dashed line) and fail (solid line) the $p_{T}$ window cut. The arrows indicate the $\chi_{K S}^{2}<20.0$ cut. In the bottom half, the $\left|z_{K S}-z_{\text {primary }}\right|$ distributions are shown similarly with the arrows indicating the $\left|z_{K S^{-}} z_{\text {primary }}\right|<3.0$ crn cut. All distributions are based $8028 K_{S}$ embedded into $|\eta|<2.0$ after passing the event cuts. The distributions are normalized to unit area. 


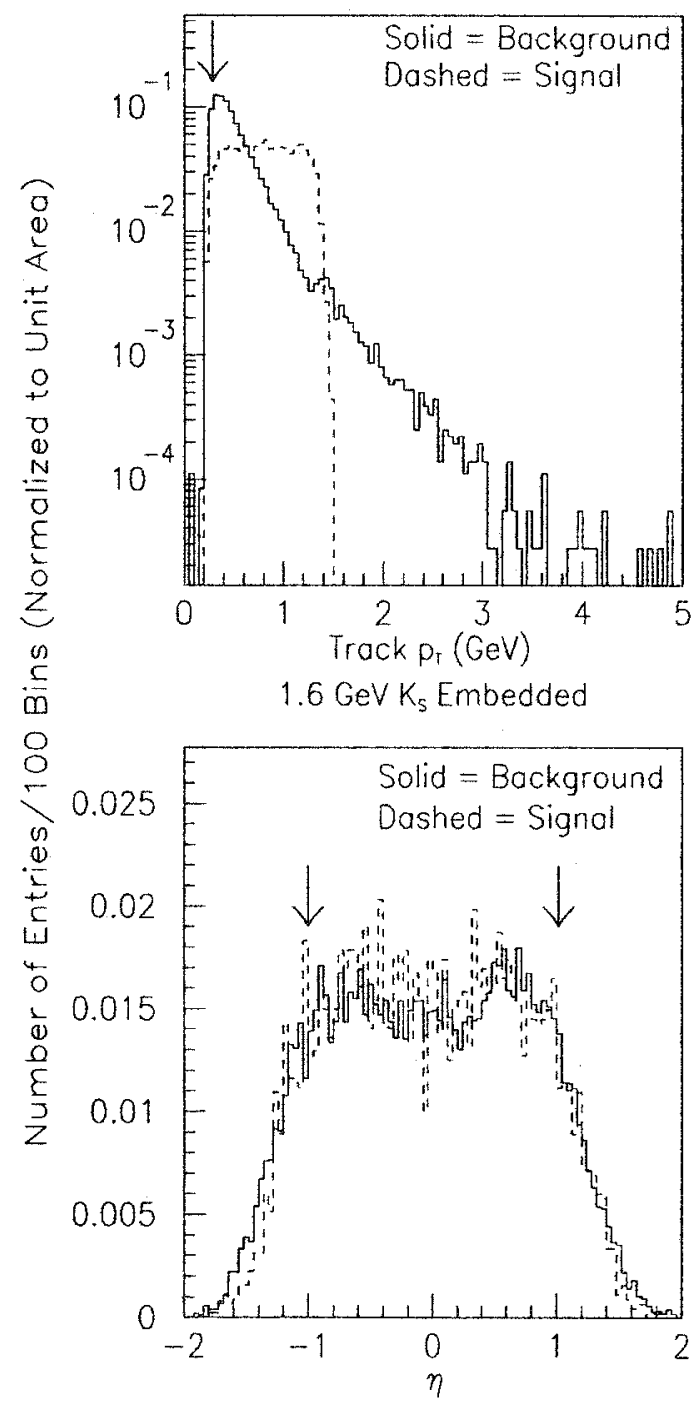

$1.6 \mathrm{GeV} \mathrm{K} \mathrm{K}_{\mathrm{s}}$ Embedded





$1.6 \mathrm{GeV} K_{\mathrm{s}}$ Embedded

Figure 4.9: In the top half, the daughter track $p_{T}$ distributions of the $1.6 \mathrm{GeV}$ (left) and $3.0 \mathrm{GeV}$ (right) candidates which pass (dashed line) and fail (solid line) the $p_{T}$ window cut. The arrows indicate the $p_{T}^{\text {tracksfrom } K S}>300.0 \mathrm{MeV}$ cut. In the bottom half, the $\eta^{K S}$ distributions are shown similarly with the arrows indicating the $\left|\eta^{K S}\right|<1.0$ cut. All distributions are based $8028 K_{S}$ embedded into $|\eta|<2.0$ after passing the event cuts. The distributions are normalized to unit area. 



Figure 4.10: In the top half, the 3D displacement ${ }^{K S}$ distributions of the $1.6 \mathrm{GeV}$ (left) and $3.0 \mathrm{GeV}$ (right) candidates which pass (dashed line) and fail (solid line) the $p_{T}$ window cut. The arrows indicate the $3 \mathrm{D}$ displacement ${ }^{K S}>1.0 \mathrm{~cm}$ cut. In the bottom half, the $2 \mathrm{D}$ pointing vector $\left(\cos \theta_{P D}^{K S}\right)$ distributions are shown with the arrows indicating the $\cos \theta_{P D}^{K S}>0.990$ cut. All distributions are based $8028 K_{S} \mathrm{em}$ bedded into $|\eta|<2.0$ after passing the event cuts. The distributions are normalized to unit area. 


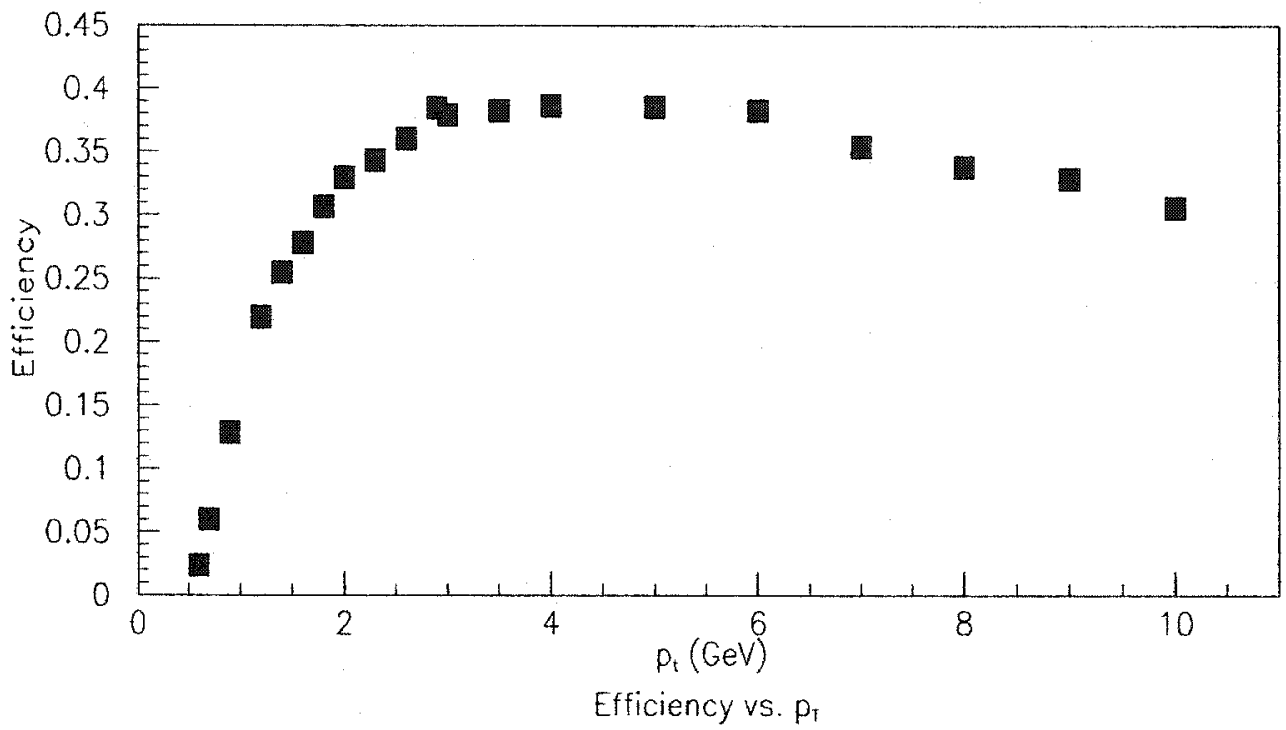

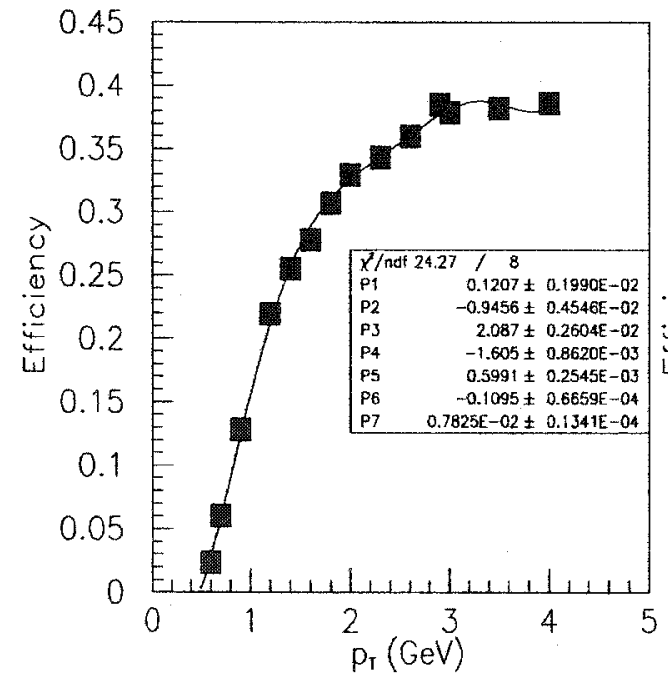

Efficiency vs. P



Figure 4.11: The $K_{S}$ efficiency vs. $p_{T}$ curve for the Minimum Bias set. Each point in the top figure represents a $p_{T}$ value which implemented track-embedding, and these points are divided into 2 distinct fit regions shown in the bottom diagrams. This efficiency curve does not implement the branching ratio correction, and the $K_{S}$ are embedded between $|\eta|<2.0$. For embedded $K_{S}$ with $p_{T}$ below $1.0 \mathrm{GeV}$, 16677 events passing the event cuts are used. For embedded $K_{S}$ with $p_{T}$ above 1.0 $\mathrm{GeV}, 8028$ events passing the event cuts are used. 


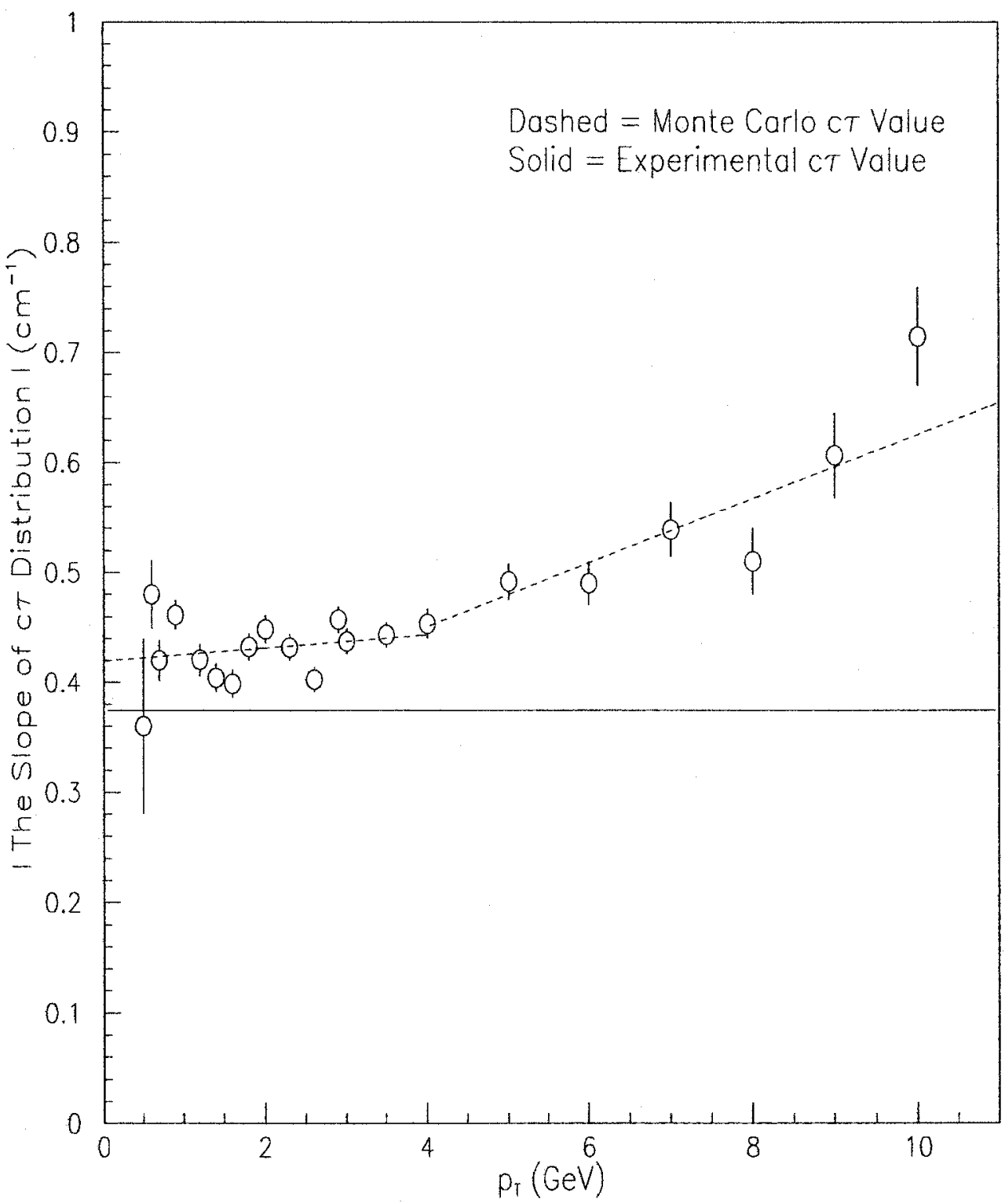

IThe Slope of $c r$ Distribution I vs. pr for $K_{s}$

Figure 4.12: The |slope of the $\mathrm{c} \tau$ distribution| vs. $p_{T}$. This curve is implemented in the $c \tau$ calculation. The solid line is the expected value, and the dashed line is the efficiency value extracted from the track embedding. (bottom right). 


\section{Chapter 5}

\section{Minimum Bias: Results}

The efficiency corrections will now be applied to the $K_{S}$ sample in order to produce plots which offer greater insight into $K_{S}$ production in Minimum Bias events. In the first section, the efficiency adjusted $\mathrm{c} \tau$ measurement will be highlighted along with its implications regarding the reliability of the Monte Carlo. Then, the mass

spectrum, the number of $K_{S}$, the uncorrected $\frac{d N_{K_{S}}}{d p_{T}}$ spectrum, the $\frac{E}{N_{\text {event }}} \frac{d^{3} N_{K S}}{d^{3} p}$ curve, the track density $\frac{d N_{K S}}{d \eta}$, the $\left\langle p_{T}\right\rangle$, and the invariant cross-section will be among a list of items to be discussed. Bear in mind, the results obtained from the Minimum Bias set are not the primary objective, but rather they are only intended to compare with previously publications.

\section{1 c $\tau$ Measurement}

The efficiency adjusted $\mathrm{c} \tau$ measurement is compared to the accepted value so that the accuracy of the efficiency corrections may be gauged. The first topic of discussion will be a review of what transpired in Chapter 4 followed by stating the unadjusted $c \tau$ value, then the implementation of the $c \tau$ efficiency is to be described, and to end this section, the efficiency corrected $c \tau$ will be presented along with what may be inferred as to the precision of the Monte Carlo and the $p_{T}$ efficiency curve. 
As in section 4.3 which pertains to $\mathrm{c} \tau$ efficiency calculation, the proper lifetime is denoted by the following equation:

$$
c \tau=\frac{M_{K S} D_{K S}}{P_{K S}}
$$

where $P_{K S}$ is the $3 \mathrm{D}$ momentum of the $K_{S}, D_{K S}$ is the $3 \mathrm{D}$ displacement of the $K_{S}$, and $M_{K S}$ is the mass of the $K_{S}$ [40] [63] [64]. Again, the experimental check is the reciprocal of the slope of the decaying exponential of the positive $c \tau$ distribution, and the accepted value is $2.6786 \pm 0.0024 \mathrm{~cm}$ for $K_{S}$ [9]. Furthermore, the $K_{S}$ are selected with the cuts employed in the $\mathrm{c} \tau$ efficiency calculation, except for the $p_{r}$ window cut, and these cuts are given below:

- Sample $K_{S}$ in $\mathrm{c} \tau$ Study

- Event Cuts

$-\chi_{K S}^{2}<20.0$

$-\left|z_{K S^{-}} z_{\text {primary }}\right|<3.0 \mathrm{~cm}$

$-p_{T}^{\text {tracks from } K S}>300 \mathrm{MeV}$

$-\left|\eta^{K S}\right|<1.0$

- 3D Displacement ${ }^{K S}>1.0 \mathrm{~cm}$

Background subtraction is likewise needed to remove background $\mathrm{c} \tau$ values from the central region $(0.48$ to $0.52 \mathrm{GeV})$. To review, the method involves adding the $\mathrm{ct}$ values from the sideband mass regions $(0.45-0.48 \mathrm{GeV}$ and $0.52-0.55 \mathrm{GeV})$, and then subtracting the combined histogram from the $\mathrm{c} \tau$ distribution containing both the $K_{S}$ and background candidates having central region $(0.48-0.52 \mathrm{GeV})$ mass values. When the sum of the sideband $c \tau$ distributions is subtracted from the central $c \tau$ 
distribution, the sum of the sidebands must be weighted with the fraction denoted by Equation 5.2 :

$$
\text { fraction }=\frac{N_{C}^{\text {total }}-N_{K S}^{\text {total }}}{N_{S 1}^{\text {total }}+N_{S 2}^{\text {total }}}
$$

where $N_{S 1}^{\text {total }}$ and $N_{S 2}^{\text {total }}$ are the total number of candidates in each of the sideband regions $S 1$ and $S 2, N_{C}^{\text {total }}$ is the total number of $K_{S}$ and background candidates in the central region, and $N_{K S}^{t o t a l}$ is the total number of $K_{S}$ in the central peak. The errors are propagated bin by bin utilizing the following equation:

$$
\text { error }=\sqrt{N_{C}^{b i n}+\text { fraction }\left(N_{S 1}^{b i n}+N_{S 2}^{b i n}\right)}
$$

After the background subtraction procedure, the resulting $\mathrm{c} \tau$ of the central region has had these background $\mathrm{c} \tau$ removed, and this is the distribution from which the slope of the decaying exponential is computed. In particular, $N_{S 1}^{\text {total }}=139769$, $N_{S 2}^{\text {total }}=132707, N_{C}^{\text {total }}=244948$, and $N_{K S}^{\text {total }}=61790$ yield a fraction of approximately 0.672 . $N_{S 1}^{\text {total }}$ is within $5 \%$ of $N_{S 2}^{\text {total }}$, and nothing is gained by having them equal. The resulting value is quite robust, and the divisions of the sidebands and central regions are not important so long as they are reasonable. Moreover, it best to keep the regions consistent with those in the $p_{T}$ calculation.

In Figure 5.1, the fitted $\mathrm{c} \tau$ distribution is shown after background subtraction and before efficiency adjustments. The proper lifetime prior to efficiency corrections is $2.3736 \pm 0.0116 \mathrm{~cm}$. The number is slightly smaller than the accepted measured value of $2.6786 \pm 0.0024 \mathrm{~cm} \mathrm{[9]} \mathrm{because} \mathrm{some} \mathrm{of} \mathrm{the} K_{S}$ with longer lifetimes are lost due to the lack of hits in inner layers of the CTC. Therefore, before the efficiency corrections, the widely accepted quantity and this experimental value differ by about 15\%. Hopefully, the $\mathrm{c} \tau$ determined with the $\mathrm{c} \tau$ efficiencies from Chapter 4 will 
improve the agreement.

The efficiency corrected $c \tau$ is generated in the identical matter as the unadjusted c $\tau$ with one exception: before booking histograms, every $\mathrm{c} \tau$ and mass value is weighted by a function of the efficiency correction from the $p_{T}$ vs. $\mathrm{c} \tau$ plot studied in Chapter 4. The number of $K_{S}$ is extracted from the weighted mass plot as is the number of background candidates in the sideband and central regions. The adding and subtracting histograms proceeds similar as before with the $N_{C}^{\text {total }}, N_{K S}^{\text {total }}, N_{S 1}^{\text {total }}$, and $N_{S 2}^{\text {total }}$ being calculated from histograms with these correction factors. There also exists a $p_{T}$ cut of $0.5 \mathrm{GeV}$ for the $K_{S}$, since below this value, the efficiencies are not well understood. In addition, all $K_{S}$ with $p_{T}>10 \mathrm{GeV}$ have adjustments equal to the efficiency at $10.0 \mathrm{GeV}$. The correction factor is expressed in Equation 5.4 :

$$
\text { weight }=\exp \left(\left(c \tau^{\text {slope }}\left(p_{T}\right)-c \tau_{\text {experimental }}^{\text {slope }}\right) * c \tau\left(p_{T}\right)\right)
$$

where $c \tau^{\text {slope }}\left(p_{T}\right)$ is taken from Figure 4.12 as a function of $p_{T}$, the $c \tau_{\text {experimental }}^{\text {slope }}$ variable is just the constant $\frac{1}{2.6786}\left(\frac{1.0}{c \tau_{\text {experimental }}}\right)$, and $c \tau\left(p_{T}\right)$ is a given $c \tau$ value to be booked.

To fill in the details, the weighted sideband values are $149900\left(N_{S 1}^{\text {total }}\right)$ and 140400 $\left(N_{S 2}^{\text {total }}\right)$ for 0.45 to $0.48 \mathrm{GeV}$ and 0.52 to $0.55 \mathrm{GeV}$, respectively, the weighted central value is $267000\left(N_{C}^{\text {total }}\right)$, the weighted number of $K_{S}$ is $71650\left(N_{K S}^{\text {total }}\right)$, and the weighted fraction is 0.67292 . The sidebands are within $7 \%$ of each other and making them to be equal would not alter the result.

The final background subtracted $\mathrm{c} \tau$ corrected for efficiencies is given in Figure 5.2 , and the efficiency corrected $\mathrm{c} r$ is computed to be $2.6882 \pm 0.0124 \mathrm{~cm}$, and the value is inline with expectations and is within errors of the experimental value of $2.6786 \pm 0.0024 \mathrm{~cm}[9]$. Other people at CDF have conducted similar lifetime 
studies and also found the experimental value to be consistent with the expected ct. First, Schub's result is within $30 \%$ of expected value with as little as $500 K_{S}$ candidates [41] [65] More recently, another study quotes the $\mathrm{c} \tau$ of $K_{S}$ as being $2.643 \pm 0.018 \mathrm{~cm}[43]$. This latter result is slightly lower than $K_{S} \quad c \tau$ derived here because the fit of [63] includes all regions of the $c \tau$ spectrum, even those, obviously departing from the exponential. The agreement indicates that the Monte Carlo accurately accounted for the decreasing $\mathrm{c} \tau$ tail of the data, and the $p_{T}$ efficiency curve may be considered to be reliably generated.

\subsection{Reconstructed Mass Peak}

Before any quantity may be computed, the $K_{S}$ sample must be obtained. As described in Chapter $3, K_{S}$ candidates have already met the following criteria:

- $K_{S}$ in $p_{T}$ sample

- Event Cuts

$-\chi_{K S}^{2}<20.0$

$-\left|z_{K S^{-}} z_{\text {primary }}\right|<3.0 \mathrm{~cm}$

$-p_{T}^{\text {tracks from } K S}>300 \mathrm{MeV}$

$-\left|\eta^{K S}\right|<1.0$

- 3D Displacement ${ }^{K S}>1.0 \mathrm{~cm}$

$-\cos \theta_{P D}^{K S}>0.990$

After these cuts have been applied, the reconstructed mass distribution appears approximately as a Gaussian resting upon a smooth background, and this is plotted 
in Figure 5.3. The mass distribution of $K_{S}$ candidates consists of a mixture of $K_{S}$ along with particles which, although they satisfy the requirements, are not $K_{S}$. Unfortunately, such background particles are indistinguishable from real $K_{S}$. With each additional cut, the number of $K_{S}$ per background $K_{S}$ may increase; however, this may reduce the number of $K_{S}$ too much. For example, an impact parameter cut would increase the Signal to Noise ratio, but it would also reduce the number of $K_{S}$ dramatically.

All the analysis from this point is based upon deriving $K_{S}$ properties from the shapes of the $K_{S}$ plus background histogram and the sideband histograms. First, the $K_{S}$ plus background histogram is fitted with a Gaussian and a 1st order polynomial. The 1st order polynomial represents the background. Then, this 1st order polynomial is subtracted from the $K_{S}$ plus background histogram shown in Figure 5.3. The resulting $K_{S}$ mass peak distribution is shown in Figure 5.4.

Ideally, the $K_{S}$ mass distribution in Figure 5.4 would be a perfect delta function. It is only the actual measurement error of the particle that gives rise to the spreading of the delta function to yield approximately a Gaussian. The fitted Gaussian in Figure 5.4 reveals the mean of the mass peak to be $0.4974 \mathrm{GeV}$ with a mass resolution of $5.293 \mathrm{MeV}$. This result agrees quite well with the world average of $0.497672 \pm 0.000031 \mathrm{GeV}[9]$. The number of $K_{S}$ candidates after cuts and unadjusted for efficiencies is evaluated by summing the number of $K_{S}$ candidates between 0.48 and $0.52 \mathrm{GeV}$ in Figure 5.4. In this case, there are $56940 \pm 441$ uncorrected $K_{S}$ where the error is taken as the Gaussian area error obtained directly from the combined Gaussian and polynomial fits. 


\section{$5.3 p_{T}$ Spectrum}

After first calculating the background subtracted $p_{T}$ curve with cuts described in the previous section, the $p_{T}$ curve is corrected with the efficiency spectrum. Using this efficiency adjusted $p_{T}$ curve, the number of $K_{S}$ in the sample can be determined.

In a procedure which closely mirrors that of the production the unadjusted $\mathrm{c} \tau$ plot, the uncorrected $p_{T}$ spectrum of the $K_{S}$ are computed via background subtraction. The technique is identical with mass ranges $(0.45$ to $0.48 \mathrm{GeV}$ and 0.52 to 0.55 for the sidebands and 0.48 to 0.52 for the central regions) and errors evaluated similarly; however, $p_{T}$ distributions are substituted for the $\mathrm{c} \tau$ distributions. Moreover, there is a slight difference in $K_{S}$ candidate selection criteria with the c $\tau$ case lacking the $2 \mathrm{D}$ pointing vector cut as well as having an additional $p_{T}$ constraint on the $K_{S}$. To be more specific, with the $p_{T}$ background subtraction, $N_{S 1}^{\text {total }}=82400$, $N_{S 2}^{t o t a l}=69169, N_{C}^{t o t a l}=159696$, and $N_{K S}^{t o t a l}=56940$ yield a fraction of approximately 0.6778 . It should be obvious that $N_{S 1}^{\text {total }}$ is $20 \%$ greater than $N_{S 2}^{\text {total }}$, and this can be resolved by altering the mass ranges for $N_{S 1}^{t o t a l}, N_{C}^{\text {total }}$ and $N_{K S}^{\text {total }}$, and $N_{S 2}^{\text {total }}$ to be 0.45 to $0.48 \mathrm{GeV}, 0.48$ to $0.515 \mathrm{GeV}$, and 0.515 to $0.55 \mathrm{GeV}$ respectively. Another solution would include slightly different weights when adding and subtracting $p_{T}$ distributions, but in the end, nothing is gained by doing so because the normalized $p_{T}$ distributions of sidebands are the same. It is also should mentioned that additional $2 \mathrm{D}$ pointing vector cut causes the asymmetry in the sidebands. The resulting unadjusted $p_{T}$ spectrum is displayed in Figure 5.5.

The next step in contimuing the understanding $K_{S}$ production is to convert the unadjusted $\frac{d N_{K S}}{d p_{T}}$ spectrum into the efficiency corrected $\frac{d N_{K S}}{d p_{T}}$ curve by applying the fitted efficiency curve obtained from Chapter 4 . To reiterate, $K_{S}$ efficiency is a function of $p_{T}$, and the function is defined with a different set of parameters for two 
distinct fit regions in the bottom of Figure 4.11. Therefore, the number of $K_{S}$ in a given bin in Figure 5.5 is multiplied by the reciprocal of the $K_{S}$ efficiency given at the value of the $p_{T}$ at the midpoint of the bin. In addition, these corrections are applied only to the $p_{T}$ region between $550 \mathrm{MeV}$ and $10 \mathrm{GeV}$ where the efficiency corrections are greater than $5 \%$ and statistics in the unadjusted bins are reasonable. Moreover, this number is multiplied by $0.6861 \pm 0.0028$ [9] to take the branching ratio into account. Although the resulting curve is not presented here because the $\frac{E}{N_{\text {event }}}$ $\frac{d^{3} N_{K S}}{d^{3} p}$ distribution is usually preferred, the number of $K_{S}$ in the adjusted curve is calculated by summing the $K_{S}$ in each bin. The number of $K_{S}$ between $550 \mathrm{MeV}$ and $10 \mathrm{GeV}$ is $250339 \pm 4984$ where the total error is the result of error propagation of each bin. The number of $K_{S}$ prior to the efficiency corrections between $550 \mathrm{MeV}$ and $10 \mathrm{GeV}$ is $56873 \pm 238$ where the error is determined statistically [41].

\subsection{The $\frac{E}{N_{\text {event }}} \frac{d^{3} N_{K S}}{d^{3} p}$ Spectrum}

The $\frac{E}{N_{\text {event }}} \frac{d^{3} N_{K S}}{d^{3} p}$ can be expressed in terms of the efficiency corrected $\frac{d N_{K S}}{d p_{T}}$ curve, and once the $\frac{E}{N_{\text {event }}} \frac{d^{3} N_{K S}}{d^{3} p}$ is obtained and fitted, variables such as $\left\langle p_{T}\right\rangle$ and $\frac{d N_{K S}}{d \eta}$ may be evaluated.

To get $\frac{E}{N_{\text {event }}} \frac{d^{3} N_{K S}}{d^{3} p}$ in terms of $\frac{d N_{K S}}{d p_{T}}$, first substitute the cylindrical coordirate form of $d p^{3}, d^{3} p=p_{T} d p_{T} d \phi d z$, to yield Equation 5.5 :

$$
\frac{E}{N_{\text {event }}} \frac{d^{3} N_{K S}}{d^{3} p}=\frac{E}{N_{\text {event }}} \frac{d^{3} N_{K S}}{p_{T} d p_{T} d \phi d z}
$$

Then, using $d \eta=\frac{d p_{z}}{E}$, Equation 5.5 can be rewritten as Equation 5.6:

$$
\frac{E}{N_{\text {event }}} \frac{d^{3} N_{K S}}{d^{3} p}=\frac{1}{N_{\text {event }}} \frac{d^{3} N_{K S}}{p_{T} d p_{T} d \phi d \eta}
$$


Since the number of $K_{S}$ are efficiency adjusted in the region of space confined by $0 \leq \phi<2 \pi$ and $|\eta|<2.0$ and the $K_{S}$ efficiency can be considered to be uniform over the variables $\phi$ and $\eta$, it follows that $d \phi=2 \pi$ and $d \eta=4$. Accordingly, in Equation $5.7, \frac{E}{N_{\text {event }}} \frac{d^{3} N_{K S . S}}{d^{3} p}$ is successfully transformed into a function of $\frac{d N_{K S}}{d p_{T}}$ :

$$
\frac{E}{N_{\text {event }}} \frac{d^{3} N_{K S}}{d^{3} p}=\frac{1}{N_{\text {event }}} \frac{d^{3} N_{K S}}{8 \pi p_{T} d p_{T}} .
$$

The $\frac{E}{N_{e v e n t}} \frac{d^{3} N_{K S}}{d^{3} p}$ spectrum in Figure 5.6 is obtained by dividing the number of $K_{S}$ in each bin of the efficiency corrected $\frac{d N_{K S}}{d p_{T}}$ curve with the $p_{T}$ value at the midpoint of the bin and by multiplying every bin with the constant $\frac{1}{8 \pi N_{\text {event }}}$ where $N_{\text {event }}=1272552$.

The $\frac{E}{N_{\text {event }}} \frac{d^{3} N_{K S}}{d^{3} p}$ spectrum is fit with a power law, and the extracted fit parameters are used to calculate important quantities such as $\frac{d N_{K S}}{d \eta}$ and $\left\langle p_{T}\right\rangle[41]$. The power law equation is given by $\frac{A p_{0}^{n}}{\left(p_{0}+p_{T}\right)^{n}}$ where $\mathrm{A}$ and $\mathrm{n}$ are free parameters and $p_{0}$ is a commonly accepted fixed constant equal to 1.3 . Furthermore, the $\frac{E}{N_{\text {event }}} \frac{d^{3} N_{K S}}{d^{3} p}$ spectrum is fitted from $0.7 \mathrm{GeV}$ to $10.0 \mathrm{GeV}$, and this is displayed in Figure 5.7. It is believed that an exponential would fit the data better for extremely low $p_{T}$; however, this analysis does not contain points in this range. In the next section, $\frac{d N_{K S}}{d \eta}$ and $\left\langle p_{T}>\right.$ will be computed.

\section{5 $\frac{d N_{K S}}{d \eta}$ and $<p_{T}>$}

The calculations of track density $\frac{d N_{K S}}{d \eta}$ and $\left\langle p_{T}\right\rangle$ will be illustrated by explaining Table 5.1. As mentioned before, the $\frac{E}{N_{\text {event }}} \frac{d^{3} N_{K S}}{d^{3} p}$ spectrum in Figure 5.7 is fitted with a power law from $0.7 \mathrm{GeV}$ to $10.0 \mathrm{GeV}$. The top of Table 5.1 displays not only the fit range and fit parameters but also the form of the power law. These fit 
parameters can then be substituted into the functions of the power law equations, to be described below, and integrated over $p_{T}$ to yield both the $\frac{d N_{K S}}{d \eta}$ and $\left\langle p_{T}\right\rangle$ quantities. By changing the limits of integration, $\frac{d N_{K S}}{d \eta}$ and $\left\langle p_{T}\right\rangle$ values may be computed for different $p_{T}$ ranges.

The integration of the product of the power law and $2 \pi p_{T}$ would compute $\frac{d N_{K S}}{d \eta}$. This is shown in Equation 5.8:

$$
\frac{d N_{K S}}{d \eta}=2 \pi\left(\int_{0.7 \mathrm{GeV}}^{10.0 \mathrm{GeV}} \frac{A p_{0}^{n} p_{T} d p_{T}}{\left(p_{T}+p_{0}\right)^{n}}\right)
$$

The fit parameters are substituted into Equation 5.8 prior to integration. In Table 5.1 , equation 5.8 is integrated from $0.7 \mathrm{GeV}$ to $10.0 \mathrm{GeV}$ as well as from $0.5 \mathrm{GeV}$ to $10.0 \mathrm{GeV}$. In the end, $\frac{d N_{K S}}{d \eta}=0.129 \pm 0.005$ for $500.0 \mathrm{MeV}<p_{T}<10.0 \mathrm{GeV}$ and $\frac{d N_{K S}}{d \eta}=0.084 \pm 0.003$ for $0.7 \mathrm{GeV}<p_{T}<10.0 \mathrm{GeV}$.

The $\left\langle p_{T}\right\rangle$ is slightly more complicated, it is given by Equation 5.9 for 0.7 $\mathrm{GeV}<p_{T}<10.0 \mathrm{GeV}:$

$$
<p_{T}>=\frac{\int_{0.7 \mathrm{GeV}}^{10.0 \mathrm{GeV}} \frac{A p_{0}^{n} p_{T}^{2} d p_{T}}{\left(p_{T}+p_{0}\right)^{n}}}{\int_{0.7 \mathrm{GeV}}^{10.0 \mathrm{GeV}} \frac{A p_{0}^{n} p_{T} d p_{T}}{\left(p_{T}+p_{0}\right)^{n}}}
$$

Again, for the $0.5 \mathrm{GeV}$ to $10 \mathrm{GeV}$ case, the limits of integration in the above equation need to be changed. The $\left\langle p_{T}>=1.022 \pm 0.005\right.$ for $500.0 \mathrm{MeV}<p_{T}<10.0$ $\mathrm{GeV}$, and the $<p_{T}>=1.251 \pm 0.005$ for $700.0 \mathrm{MeV}<p_{T}<10.0 \mathrm{GeV}$. The errors are propagated with approximations in order to reduce the number of terms.

One way to compare the results with others such as Schub [41] and Rimondi, Moggi, and Deninno [43] is to study the $\left\langle p_{T}>\right.$ over all $p_{T}$. The previous analyses cite $\left\langle p_{T}\right\rangle=0.60 \pm 0.03[41]$ and $\left\langle p_{T}\right\rangle=0.61 \pm 0.02[43]$. Over all $p_{T}$, the number here is within their errors, $\left\langle p_{T}\right\rangle=0.59 \pm 0.005$. There are different methods of fitting the $\frac{E}{N_{\text {event }}} \frac{d^{3} N_{K S}}{d^{3} p} \quad$ spectrum, and the method utilized for this comparison consists of 
fixing $p_{0}=1.3$ and proceeding as described in this section. In addition, Schub [41] computes $\frac{d N_{K S}}{d \eta}$ over all $p_{T}$ to be $0.29 \pm 0.04$, and in this analysis, $\frac{d N_{K S}}{d \eta}=0.30 \pm 0.01$. Hence, these results are all within $5 \%$ of each other.

It is important to mention that there are many other fitting techniques for methods $\frac{E}{N_{\text {event }}} \frac{d^{3} N_{K S}}{d^{3} p} \quad$ spectra. First, $p_{0}$ does not necessarily need to be constant. In [43], when it is not, $p_{0}$ increases to $3.16 \pm 0.06$ (as opposed to $p_{0}=1.3$ ), and $\left\langle p_{T}\right\rangle$ over all $p_{T}$ increases to $0.74 \pm 0.07$. Moreover, the power law may be the only function to fit the $\frac{E}{N_{\text {event }}} \frac{d^{3} N_{K S}}{d^{3} p}$. For example, an exponential may be implemented with or without a power law. If both an exponential and a power law are used, below a given $p_{T}$, the exponential fits the spectra, and above a transition point, a power law may be used. This transition point need not be where data exists as long as continuity of the functions and their derivatives is enforced. This $\left\langle p_{T}\right\rangle$ depends tremendously on the systematics of the given method, and [41] is an excellent reference to continue this discourse. However, the point is not focus exclusively on the $K_{S}$ production in Minimum Bias events. Hence, with the agreement with previous published $\left\langle p_{T}\right\rangle$ and $\frac{d N_{K S}}{d \eta}$ for the case of fixing $p_{0}$, fitting the $\frac{E}{N_{\text {event }}} \frac{d^{3} N_{K S}}{d^{3} p}$ with a power law, and integrating the fit over all $p_{T}$ being sufficient, contrasting the invariant cross-section with these analyses will be described in the subsequent section.

\subsection{Invariant Cross-Section}

The invariant cross-section will be the last topic. This involves multiplying the $\frac{E}{N_{\text {event }}} \frac{d^{3} N_{K S}}{d^{3} p}$ spectrum by the $\sigma_{\text {effective. }}$. For $p \bar{p}$ collisions, the $\sigma_{\text {effective }}=43 \pm 6 \mathrm{mb}$. In Figure 5.8, [41] overlaps the invariant cross-section computed in this thesis, and the two curves are within $5 \%$ of each other. Also, the explicit values are compared for various $p_{T}$ in Table 5.6. The agreement is approximately $15 \%$ on a point-by- 
point basis except when $p_{T}>3.0 \mathrm{GeV}$ where statistics are limited [41]. Moreover, the cross-section measurement from [43] is quite comparable. Since the consistency with a few Minimum Bias $K_{S}$ measurements has been shown, the $K_{S}$ production in jets will studied next. 
Table 5.1: $\frac{d N_{K S}}{d \eta}$ and $\left\langle p_{T}\right\rangle$ for $K_{S}$ in the Minimum Bias set.

\begin{tabular}{|c|c|}
\hline Fit Range & $700 \mathrm{MeV}<p_{T}<10.0 \mathrm{GeV}$ \\
\hline Function Fitted & $\frac{A p_{0}^{n}}{\left(p_{0}+p_{T}\right)^{n}}$ \\
\hline Resulting & $A=0.991 \pm 0.030$ \\
Fit Parameters & $n=7.427 \pm 0.037$ \\
& $p_{0}=1.3($ fixed) \\
\hline$\frac{d N_{K S}}{d \eta}$ from $0.5<p_{T}<10.0(\mathrm{GeV})$ & $0.129 \pm 0.005$ \\
$\frac{d N_{K S}}{d \eta}$ from $0.7<p_{T}<10.0(\mathrm{GeV})$ & $0.084 \pm 0.003$ \\
$<p_{T}>$ from $0.5<p_{T}<10.0(\mathrm{GeV})$ & $1.022 \pm 0.005$ \\
$\left\langle p_{T}>\right.$ from $0.7<p_{T}<10.0(\mathrm{GeV})$ & $1.251 \pm 0.005$ \\
\hline
\end{tabular}

Table 5.2: The invariant cross-section comparison .

\begin{tabular}{|c|c|c|}
\hline$p_{T}(\mathrm{GeV})$ & $\begin{array}{c}\text { Schub's [41] } \\
\mathrm{E} \frac{d^{3} \sigma}{d^{3} p}\left(\mathrm{mb} / \mathrm{GeV}^{2}\right)\end{array}$ & $\begin{array}{c}\text { Derived Here } \\
\mathrm{E} \frac{d^{3} \sigma}{d^{3} p}\left(\mathrm{mb} / \mathrm{GeV}^{2}\right)\end{array}$ \\
\hline 0.85 & $0.900 \pm 0.200$ & $0.915 \pm 0.058$ \\
1.15 & $0.310 \pm 0.050$ & $0.350 \pm 0.019$ \\
1.45 & $0.180 \pm 0.020$ & $0.159 \pm 0.009$ \\
1.75 & $0.065 \pm 0.009$ & $0.075 \pm 0.005$ \\
2.05 & $0.032 \pm 0.006$ & $0.030 \pm 0.003$ \\
2.35 & $0.019 \pm 0.004$ & $0.022 \pm 0.002$ \\
2.65 & $0.009 \pm 0.002$ & $0.010 \pm 0.002$ \\
2.95 & $0.005 \pm 0.002$ & $0.006 \pm 0.001$ \\
3.30 & $0.003 \pm 0.001$ & $0.003 \pm 0.0008$ \\
3.75 & $0.0004 \pm 0.0005$ & $0.002 \pm 0.0008$ \\
4.50 & $0.0004 \pm 0.0002$ & $0.0007 \pm 0.0002$ \\
5.50 & $0.0002 \pm 0.0001$ & $0.00002 \pm 0.00008$ \\
7.00 & $0.00009 \pm 0.00006$ & $0.00003 \pm 0.00004$ \\
\hline
\end{tabular}




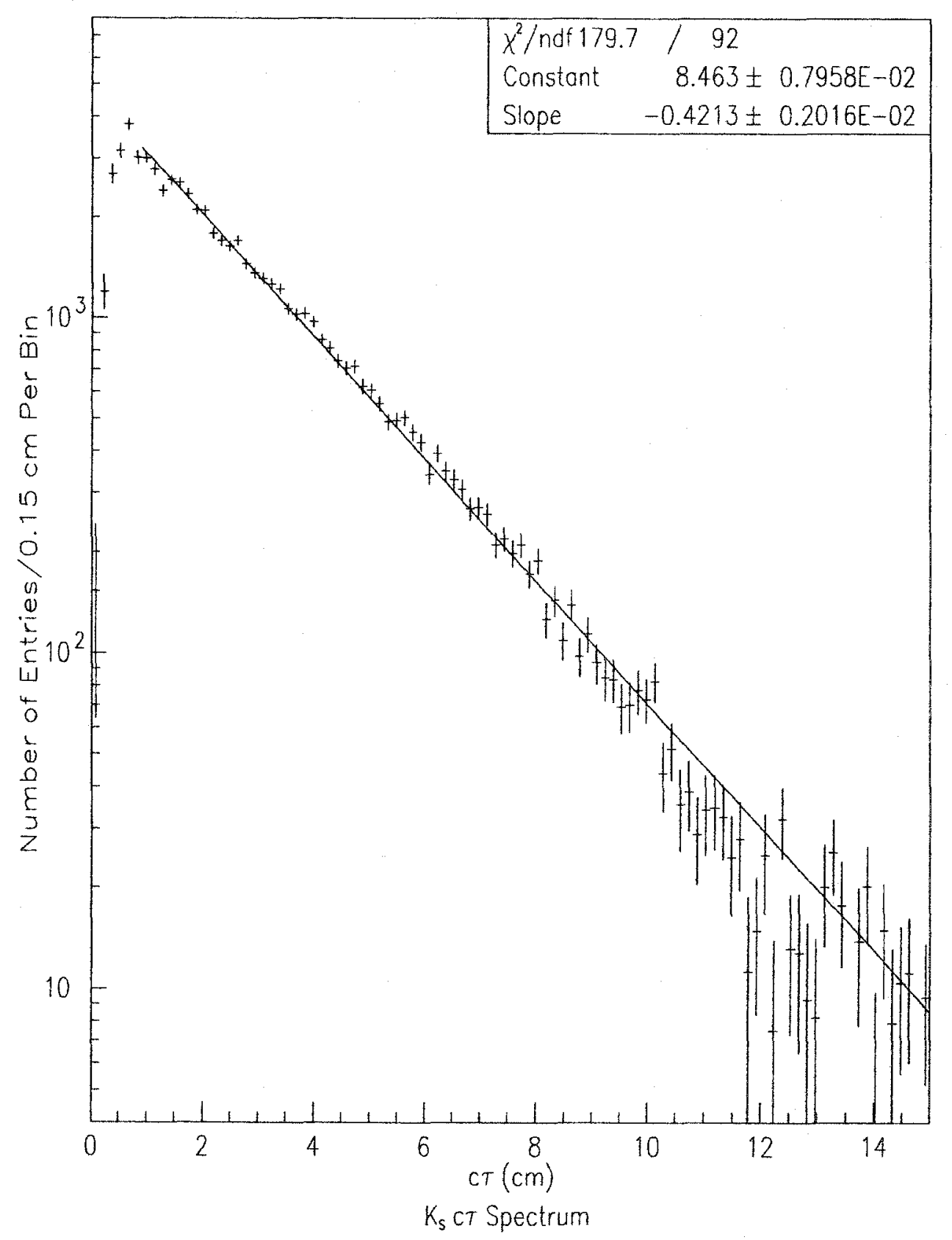

Figure 5.1: The $K_{S} \mathrm{c} \tau$ spectrum. The curve is obtained after background subtraction and is not corrected for efficiencies. 


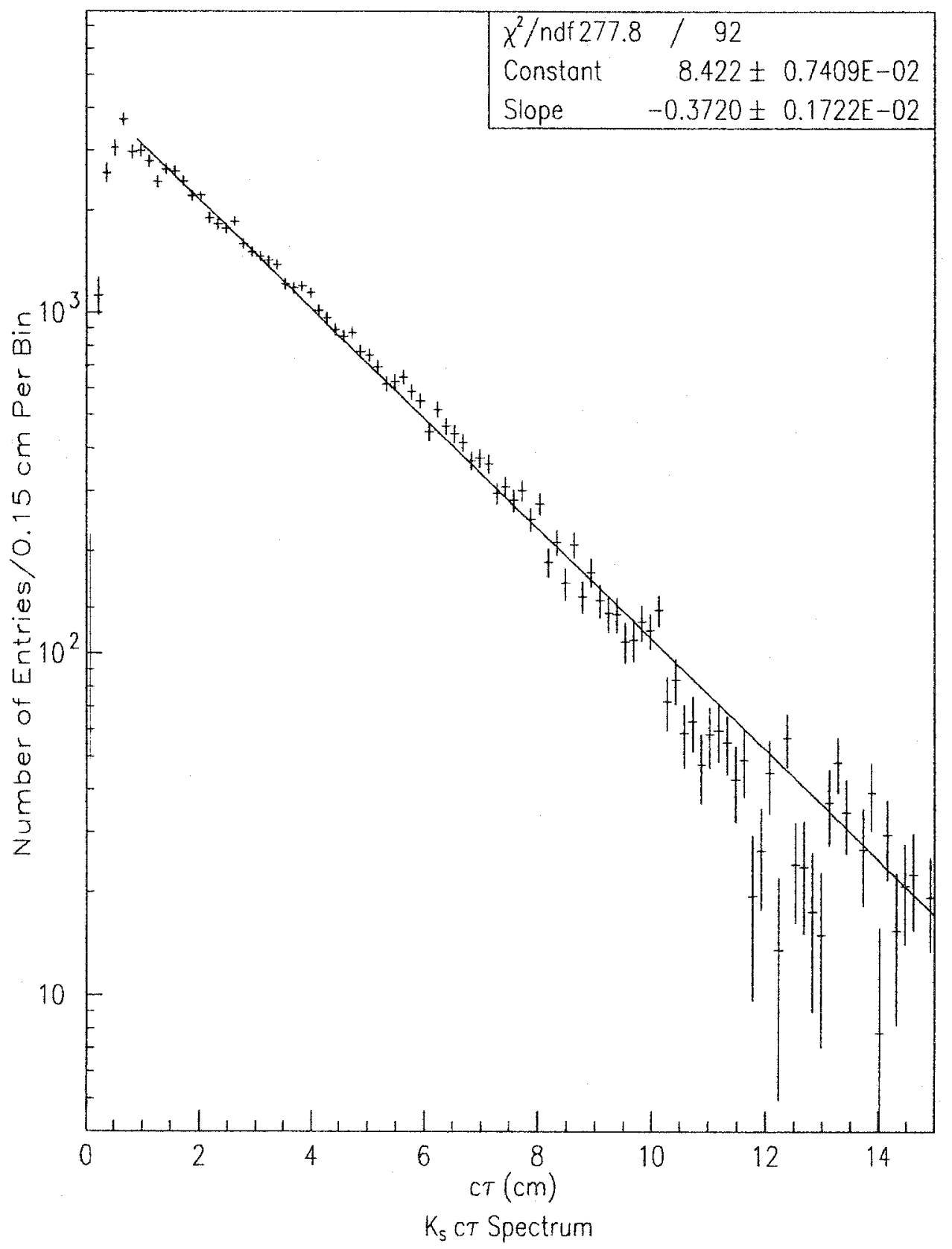

Figure 5.2: The $K_{S}$ c $\tau$ spectrum. The curve is obtained after background subtraction and is corrected for efficiencies. 




Figure 5.3: The mass distribution of $K_{S}$ candidates before background subtraction and after Cuts. 


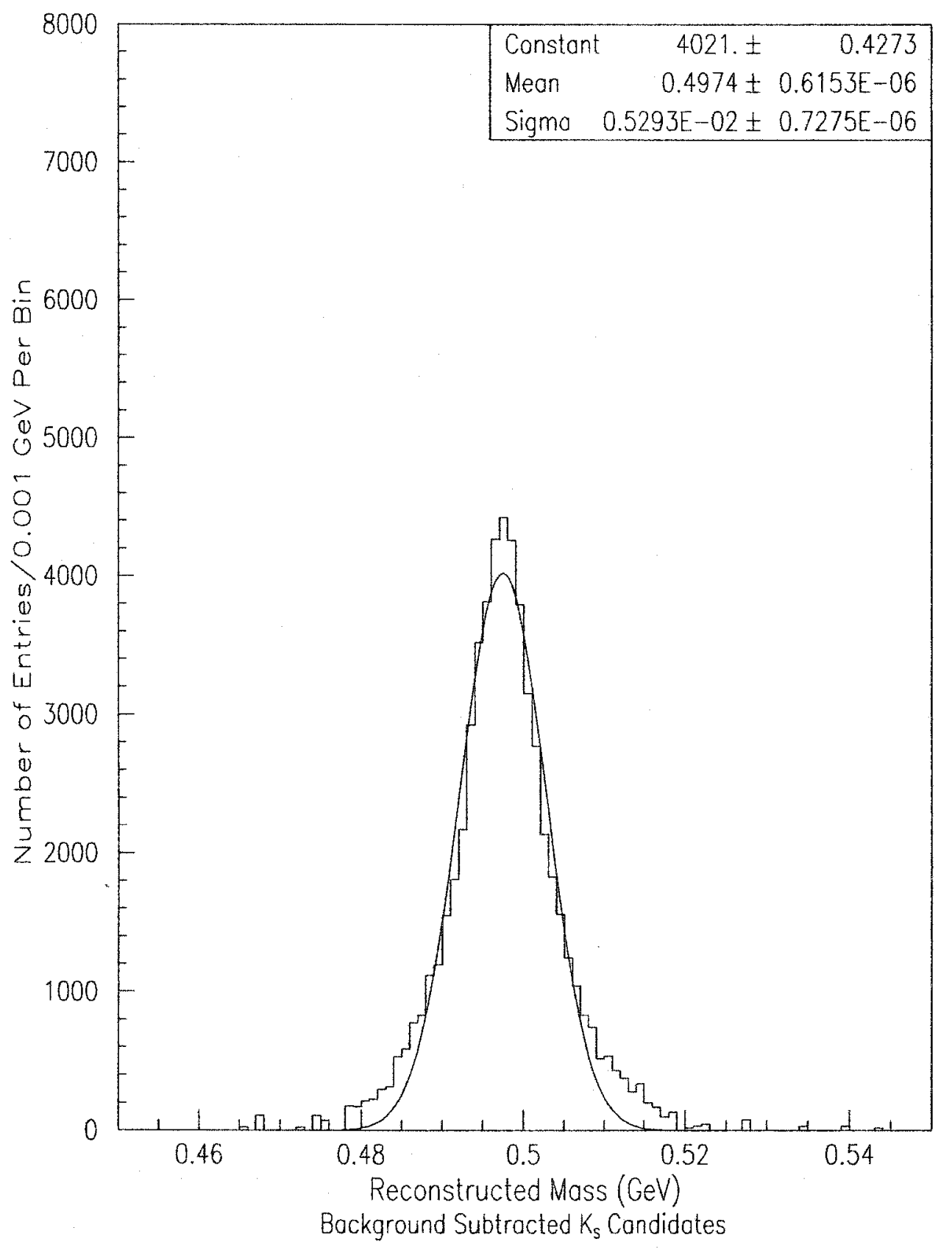

Figure 5.4: The mass distribution of $K_{S}$ candidates after background subtraction and after cuts. 


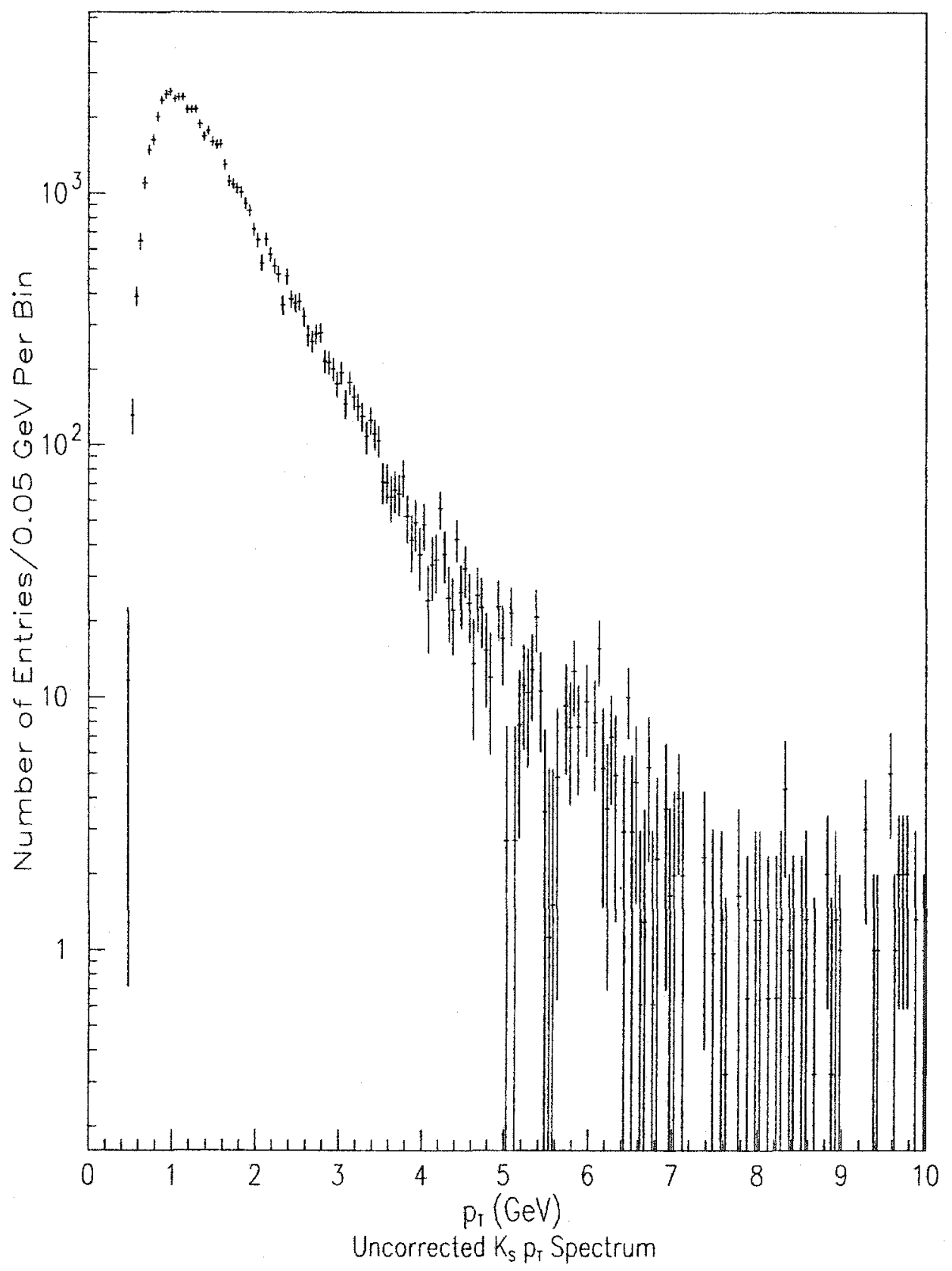

Figure 5.5: The $K_{S} \frac{d N}{d p_{T}}$ spectrum. The curve is obtained after background subtraction and is not corrected for efficiencies. 


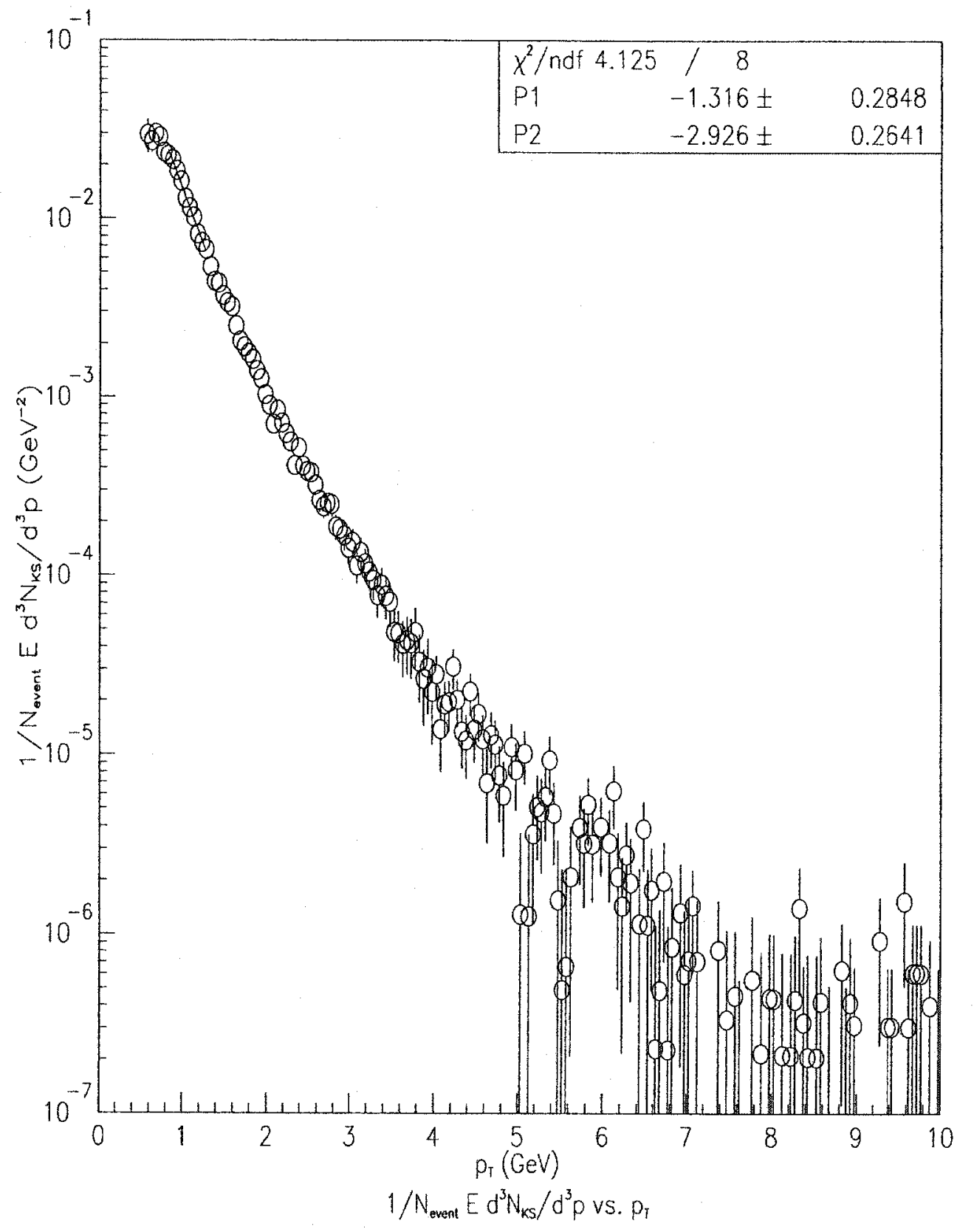

Figure 5.6: The $\frac{E}{N_{\text {event }}} \frac{d^{3} N_{K S}}{d^{3} p}$ spectrum. The spectrum is obtained after background subtraction and is corrected for efficiencies. The curve is fitted with an exponential. 


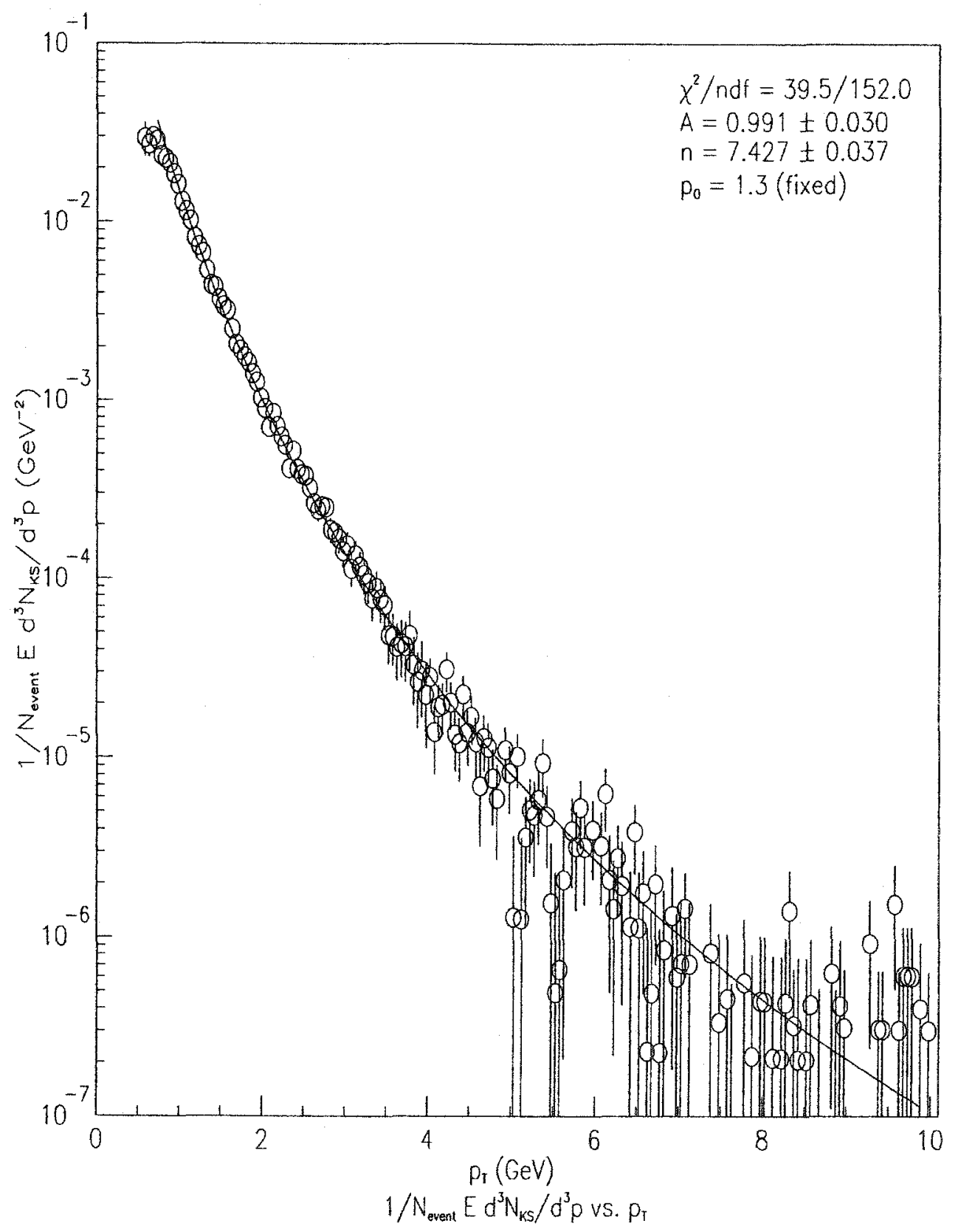

Figure 5.7: The $\frac{E}{N_{e v e n t}} \frac{d^{3} N_{K S}}{d^{3} p}$ spectrum. The spectrum is obtained after background subtraction and is corrected for efficiencies. The curve is fitted with a power law from $0.7 \mathrm{GeV}$ to $10.0 \mathrm{GeV}$. 


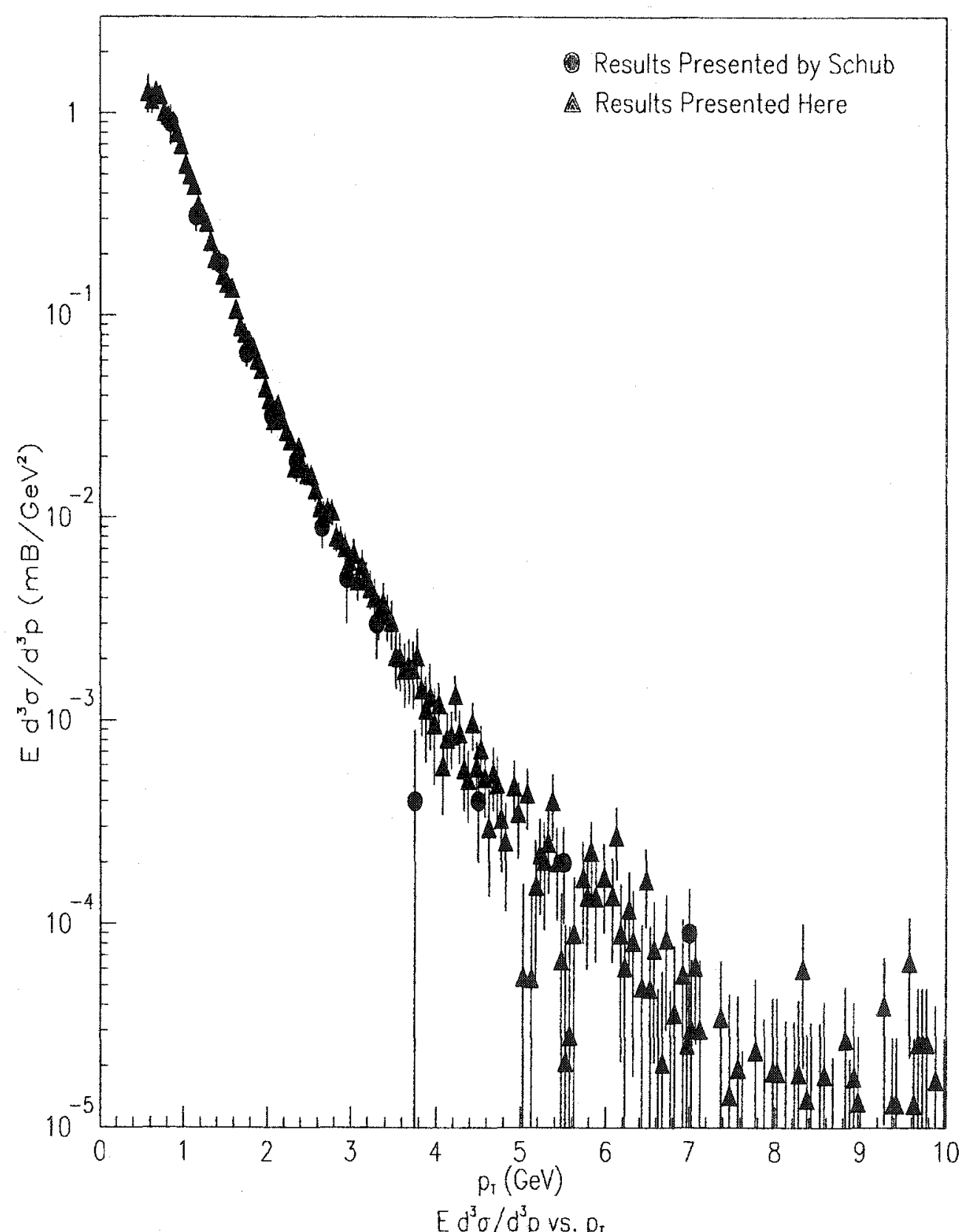

Figure 5.8: The $\frac{E d^{3} \sigma}{d^{3} p}$ spectrum for $K_{S}$. The curve is obtained after background subtraction and is corrected for efficiencies. 


\section{Chapter 6}

\section{Jets: Selection Criteria}

The same Minimum Bias event selection cuts are applied to the jet events that pass the jet triggers. The good run cut, the primary vertex cut, and the cosmic filter are employed. The cosmic filter will be described more thoroughly here because the effects are more prevalent. In addition, there is a jet trigger cut that is necessary to prevent double counting of jet events from different jet data samples. The jet selection as well as $K_{S}$ selection will also be discussed.

\subsection{Event Selection}

As mentioned previously, the bad run cut, the primary vertex cut, the cosmic filter, and jet trigger cuts are used. Since only the last two cuts are not discussed in the "Minimum Bias Event" chapter, the effects of the cosmic filter and the jet trigger cuts will be described here.

\subsubsection{Cosmic Filter}

The idea behind the cosmic filter is to reduce the ntuple size by eliminating events that do not appear to be $p \bar{p}$ collisions. The effectiveness of the filter scales with energy; hence, the filter is negligible for the Minimum Bias events and is most 
effective for events that pass the jet70 and jet100 triggers. It would be good for the filter to remove bad events with bad jets without significantly altering the number of $K_{S}$ found inside jets as well as the $\frac{N_{K S}}{j e t}$ values.

The cosmic filter looks for more than $6.0 \mathrm{GeV}$ of energy in the hadronic calorimeters that is "out-of-time" from the CTC readout. In addition to vetoing cosmic events, monojet, Main Ring splash, and dijets with small missing $E_{T}$ are removed from the sample. Others studying the effect of the cosmic filter records that $99.75 \%$ of the jet 20 events, $90.11 \%$ of the jet 50 events, $77.74 \%$ of the jet 70 events, and $77.91 \%$ of the jet100 events pass the cut without altering the jet $E_{T}$ spectrum. Taking this into account, only a study is preformed on the jet100 set since this would give an upper limit the effect.

Of 52025 jet100 events, 43699 events ( $84 \%$ of the total number of events in this small sample) pass the cosmic filter. Dividing the jets into three energy ranges (>100 GeV,50-100 GeV, and 20-50 GeV), Tables 6.1,6.2, and 6.3 show the effect the cosmic filter has on the number of jets, the number of $K_{S}$ in jets, and $\frac{N_{K S}}{j e t}$. The cosmic filter cuts less than $6 \%$ of the jets, less than $2 \%$ of the $K_{S}$, and tends to increase the $\frac{N_{K S}}{j e t}$ by at most $6 \%$. All $K_{S}$ in the sample must be between 1-10 $\mathrm{GeV}$ and $|\eta|<1.0$. The errors on the number of the $K_{S}$ and the $\frac{N_{K S}}{j e t}$ are all less than $5 \%$. Overall, the $\frac{N_{K S}}{j e t}$ ratio increases at most $5 \%$ because of the cosmic filter and is believed to be the result of not the rejection of $K_{S}$ but rather the reduction of bad jets in bad events [38] [39]. In Tables 6.1-6.3, the effect of the cosmic filter is shown on the number of jets, the number of $K_{S}$ inside jets, and the $\frac{N_{K S}}{j e t}$ for 20-50 $\mathrm{GeV}, 50-100 \mathrm{GeV}$, and greater than $100 \mathrm{GeV}$ jets, respectively. 
Table 6.1: The number of jets, $K_{S}$ in jets, and $\frac{N_{K S}}{j e t}$ in $20-50 \mathrm{GeV}$ jets in the Jet100 sample before and after the cosmic filter.

\begin{tabular}{|c|c|c|c|}
\hline $20-50 \mathrm{GeV}$ & Before & After & $\%$ \\
\hline Jets & 9848 & 9469 & 96.2 \\
$K_{S}$ in Jets & 1222 & 1209 & 98.9 \\
$\frac{N_{K S}}{\text { jet }}$ & 0.1223 & 0.1290 & 105.5 \\
\hline
\end{tabular}

Table 6.2: The number of jets, $K_{S}$ in jets, and $\frac{N_{K S}}{j e t}$ in $50-100 \mathrm{GeV}$ jets in the Jet100 sample before and after cosmic filter.

\begin{tabular}{|c|c|c|c|}
\hline $50-100 \mathrm{GeV}$ & Before & After & $\%$ \\
\hline Jets & 16001 & 15743 & 98.4 \\
$K_{S}$ in Jets & 2054 & 2042 & 99.6 \\
$\frac{N_{K S}}{\text { jet }}$ & 0.1276 & 0.1300 & 101.9 \\
\hline
\end{tabular}

Table 6.3: The number of jets, $K_{S}$ in jets, and $\frac{N_{K S}}{j e t}$ in $>100 \mathrm{GeV}$ jets in the Jet100 sample before and after cosmic filter.

\begin{tabular}{|c|c|c|c|}
\hline$>100 \mathrm{GeV}$ & Before & After & $\%$ \\
\hline Jets & 30947 & 29336 & 94.8 \\
$K_{S}$ in Jets & 3469 & 3455 & 99.6 \\
$\frac{N_{K S}}{\text { jet }}$ & 0.1120 & 0.1178 & 105.2 \\
\hline
\end{tabular}




\subsubsection{Trigger Cut}

Since the jet sample actually consists of 4 different tape samples with 5 different L3 triggers, it is not uncommon that one event to be recorded in one or more data sets. In fact, about $15 \%$ of the events which pass the jet70 trigger can also be found in the either the jet20, jet50, or jet100 data sets. One might suspect that every event which satisfies the jet100 trigger would automatically pass the jet20 trigger, but because of prescaling, this is often not the case and is not as straightforward.

For this double counting to avoided, the trigger bit string for each event is examined. A sequence of bit values of 0 in the string becomes equal to 1 if an event passes a particular trigger; the string is quite long because there are dozens of possible triggers. To simplify, let us say an event has a bit string denoted by 1111 $(1=$ pass jet20, 1 = pass jet50,1 = pass jet70, and $1=$ pass jet100) passes all of the jet triggers. If the event passes only the jet20 and jet50 triggers, the string is equal to 1010 . There are few different algorithms that would be just as effective to rid the sample of double-counted events, all are equivalent because there is only a unique set. By vetoing events based upon there L3 bit pattern, the resulting data sample contains only unique events. It is important to note this not a "real" cut that removes any events but rather just those that appear more than once [48].

\subsection{Jet Selection}

When a quark or a gluon fragments, many particles directed toward a particular direction are contained within a cone. Ideally, if all particles are contained within this cone, all momentum and energy of the jet are equal to that of the initial fragmented quark or gluon. In order to account for as much of the energy of the 
initial quark or gluon, all particles, charged and neutral, need to be accounted for. Hadronic and electromagnetic energy clusters in the calorimeter can be correlated to that of the energy of the initial fragmented quark or gluon to yield an object called a "jet". A jet is a very well-studied entity and is just as real a physics object as a photon, electron, or muon, except the exact definition is a matter of constant debate and varies much more from analysis to analysis.

A given jet is characterized by the clustering algorithm implemented. A good place to begin is with the cone size of a jet, $\Delta R$, and this variable is defined in units of $\eta$ and $\phi$ by $\Delta R=\sqrt{\Delta \eta^{2}+\Delta \phi^{2}}$. Some people prefer $\Delta R=1.0$, others like $\Delta R=0.4$, but the most common at CDF is $\Delta R=0.7$. In this analysis, $K_{S}$ must be within a jet having a cone size of 0.7 .

The idea of jet clustering is to group energy towers together to form bigger and bigger clusters of energy within a cone of 0.7 until all energy towers are associated with either itself or another cluster. The cluster algorithm firsts lists all the towers in both the hadronic and electromagnetic calorimeters having $E_{T}>1.0 \mathrm{GeV}$. The largest cluster is then taken as the center of a circle having radius of $\Delta R=0.7$. If there are other towers with $E_{T}>1.0 \mathrm{GeV}$ confined within this circle, this energy is grouped with the initial seed energy and the procedure is repeated until all towers are accounted for. Whenever an energy cluster does not have any additional clusters within the 0.7 cone, the highest energy tower is selected as the new seed.

After this initial stage of the clustering process, various clusters may either be within the cone size of other clusters or overlap with other clusters. Some neighboring clusters are two distinct jets, others should be merged into one. The manner in which this is done is to assign a 4 -vector to every tower so that the direction of the 4-vector points from the event vertex to the center of the towers 
shower maximum and the magnitude is that of the deposited energy of the tower. The 4-vectors components are summed together according to the following equations

$$
\begin{gathered}
E_{x}=\sum E_{x}^{i}, E_{y}=\sum E_{y}^{i}, E_{z}=\sum E_{z}^{i}, E=\sum E^{i} \\
E_{T}=E \sin \theta, \eta=-\ln \left(\tan \frac{\theta}{2}\right), \phi=\tan ^{-1} \frac{E_{y}}{E_{x}}
\end{gathered}
$$

The 4-vectors of each cluster of towers are calculated to give the value of the centroid of every cluster. After the centroids are identified, a circle radius of $\Delta R=$ 0.7 is constructed with the centroid as the center. If other clusters are within the circle of another centroid, they either need to be separated or merged together. If the overlap function (shared $\frac{E_{T}}{m i n\left(E_{1}, E_{2}\right)}$ ) is less than 0.75 , the two clusters are distinct and overlapping towers are associated with the cluster nearest in $\eta$. If either the overlap function is greater than 0.75 or one cluster is completely within the $\Delta R$ of another cluster, the clusters are merged. Centroids are then recalculated as clusters are merged or separated until a jet has the same stable set of towers [6] [7] [8] [66] .

It is worth mentioning that clusters identified as photons and electrons can be taken out of the jet energy calculations either directly or before a reclustering process. The jets in this sample do not do this because the number of photons and electrons is relatively small compared to the number of jets. Muons, depositing little energy in the calorimeters, are not taken into account [6] [7] [8].

\subsubsection{Jet Energy Corrections}

Clustering itself is insufficient to correctly determine the transverse energy of a jet. Some extra energy from other sources finds its way into the 0.7 cone (underlying 
events/multiple interaction corrections), and some energy from the parent-parton escapes the 0.7 cone (out-of-cone corrections). In addition, there are calorimeter energy response questions needed to be applied. Charged particle trajectories bent by the magnetic field so that they lie outside the jet cone are taken into account at this time.

The absolute response correction adjusts for the central calorimeter response to charged and neutral particles. By tuning Monte Carlo to data, a given cluster with a given $p_{T}$ from the data is compared to the $p_{T}$ of particles lying within a cone in the Monte Carlo. To compute the mean jet response as a function of $E_{T}$ for various cone sizes, a quadratic spline is applied for parameterization. In this analysis, the default absolute scalings are implemented [6] [7] [8] [67].

The relative response corrections refer to the scaling of the forward and plug energies to be the equivalent of being measured by the central calorimeters. This correction is obtained from jet events with one central jet and a second jet located elsewhere in the calorimeter. The imbalance between the two jets as a function of $p_{T}$ and $\eta$ of the second jet yields a correction factor. Once the jet energy is rescaled to central calorimeter, the jet energy is then adjusted using the response of the central calorimeter [6] [7] [8] [68].

As for extra energy inside the 0.7 cone not originating from the parent-parton, a quantity of energy is removed from every jet tower. The energy has two sources: 1) underlying event energy from spectator partons and 2) energy associated with multiple interactions. The first is isotropically distributed throughout the detector, and the correction factor is determined by employing a dijet sample. The second is a linear function of the number of event vertices as derived from the calorimeter information from the minimum bias samples. The average value of underlying event 
contamination is $1.01 \mathrm{GeV}$. In addition, multiple interactions also contribute extra energy inside the cone. Both are relatively independent of jet $E_{T}$ for fixed cone size.

The above corrections can be parameterized using the following equation:

$$
E_{T}^{\prime}=A\left(R\left(E_{T}, \eta\right)\right) E_{T}-n_{V T X} b-1.6 a
$$

where

$E_{T}^{\prime}=$ corrected transverse jet energy

$E_{T}=$ initial transverse jet energy

$R\left(E_{T}, \eta\right)=$ relative jet corrections

$n_{V T X}=$ number of vertices from extra interactions

$b=$ average energy for a given jet per vertex

$A\left(R\left(E_{T}, \eta\right)\right)=$ absolute energy correction

$a=$ average energy from a primary interaction as well as additional interaction energy without an additional vertex.

1.6 accounts for detector response.

The out-of-cone energy adjusts for energy of the parent parton lost from the cone due to fragmentation effects and soft gluon radiation. Since jets tend to be more collimated at higher energies, the out-of-cone energy correction added to each jet decreases with cone-size and jet $E_{T}$. The parameterization is obtained from Monte Carlo data and is given by the following equation where a,b,c depend on cone-size:

$$
E_{T}^{\prime \prime}=E_{T}^{\prime}+a\left(1-b e^{-c} E_{T}^{\prime}\right)
$$

where

$E_{T}^{\prime \prime}=$ corrected transverse jet energy for out-of-cone losses. 
$E_{T}^{\prime}=$ corrected transverse jet energy for underlying events, response, and multiple interactions.

$a=8.382$

$b=0.846$

$c=0.00740$

There is another set of jet energy corrections not incorporated into this analysis, but common enough to deserve mentioning. In cases where muons, electrons, and photons are identified, these objects can be taken out of the jet energy calculation either directly from the energy of the jet or from the energy of towers associated with its cluster. However, the number of electrons, photons, and muons objects candidates is less than $5 \%$ of the events in the jet data samples and this small effect is does not warrant this energy correction in either of its forms [6] [7] [8] [69] [70].

In Figure 6.2, the number of jets is histogramed as a function of jet $E_{T}$ for each trigger sample, both before and after jet energy corrections.

\subsubsection{Jet Vertex Selection}

To avoid having $K_{S}$ satisfying the criteria of being inside a jet cone, but in fact may be displaced along the $z$-axis, some constraint on the primary and jet vertex is necessary. Having already calculated the primary vertex, the tracking information is applied to compute the jet vertices. Each track in the CTC, within $\left|\eta_{\text {detector }}\right|<1.1$, with transverse momenta between $0.200 \mathrm{GeV} / \mathrm{c}$ and $250 \mathrm{GeV} / \mathrm{c}$, is checked to see whether it falls inside a $\Delta R=0.7$ jet cone. The $z_{0}$ values of these tracks contained within a given jet are averaged to yield a single jet vertex. Every track beyond 60 $\mathrm{cm}$ from this jet vertex are removed and the average is recalculated. This process is repeated until all tracks are within $33.75 \mathrm{~cm}$ of the jet vertex or until 3 iterations 
of the procedure. The root mean square (RMS) of the distribution tracks about the jet vertex indicates the quality of the jet vertex [71] [72].

As for the resolution, by comparing the computed jet vertices to vertices of the two electrons from $Z$ boson events, it was shown the jet vertex resolution increases with the jet energies. For example, for $E_{T}$ cuts of $10,15,20$, and $40 \mathrm{GeV}$, the jet vertex resolutions were $0.87,0.85,0.72$, and $0.54 \mathrm{~cm} \mathrm{[8]}$.

Only one primary vertex is selected as the origin of the $K_{S}$ production, and $K_{S}$ efficiencies drop significantly as $K_{S}$ are produced further and further away from the primary vertex. Hence, the jet vertex must be within $5.0 \mathrm{~cm}$ of the primary vertex. In addition, a good quality cut on the jet vertex is that it has a $z_{j e t}^{r m s}<5,0$ $\mathrm{cm}$.

There are a group of tables that render greater details of how the cuts effect the number of events and jets for each trigger sample. First, in Table 6.5, the number of events after each cut is tallied. Approximately $8-15 \%$ of the events are lost due to these cuts. As for the effect of the selection criteria on the number of jets in each sample, Tables 6.5-6.8 record the number of jets and the fraction of jets left after each cut for each jet sample. Where there are good statistics, about $25 \%$ of the $20-50 \mathrm{GeV}$ jets, $30-40 \%$ of the $50-100 \mathrm{GeV}$ jets, and $35-45 \%$ of the $100-150 \mathrm{GeV}$ jets remain after the cuts. On the bottom of the Figure, 6.3 , the difference between the primary and jet vertices is plotted for jet 50 sample.

\section{3 $K_{S}$ in Jet Selection Criteria}

The cuts are similar to those in the "Minimum Bias Selection" chapter with the exception that $K_{S}$ must be within a 0.7 cone of a jet having its vertex within 5.0 $\mathrm{cm}$ of the primary vertex. For the jet vertex to be more credible, the root mean 
square of this calculation must be less than $5.0 \mathrm{~cm}$. In addition, a $0.5 \mathrm{GeV}$ track cut replaces the $300 \mathrm{MeV}$ track cut because $K_{S}$ originated inside jets have much higher $p_{T}$ than $K_{S}$ found in Minimum Bias events. Also, the 2D pointing vector cut is reduced from 0.995 to 0.990 since low $p_{T} K_{S}$ in jets have lower $2 \mathrm{D}$ pointing vector values than $K_{S}$ found in the Minimum Bias set. The $K_{S}$ inside jets are further divided into $20-50 \mathrm{GeV}, 50-100 \mathrm{GeV}$, and $100-150 \mathrm{GeV}$ jet energy ranges.

In Tables 6.9-6.12, the total number of $K_{S}$ and the number of $K_{S}$ inside 20-50, $50-100,100-150 \mathrm{GeV}$ jets is recorded after each cut for each jet sample. Moreover, the fraction of the remaining $K_{S}$ after in each cut is shown in Figure 6.1 for all data sets. The case for all $K_{S}$ is plotted in the upper left corner; $K_{S}$ inside 20-50, 50-100, and 100-150 GeV jets are plotted in the upper right corner, the lower left corner, and the lower right corner, respectively. Later, in Chapter 7 , the effect of the cuts on the data and the Monte Carlo will be compared. Finally, the cosine of the angle between the $K_{S}$ inside a jet and the jet axis is plotted on the top of Figure 6.3. Notice that the $K_{S}$ are mostly within $30^{\circ}$ of the jet axis.

In Figure 6.4, the uncorrected reconstructed mass spectra of the $K_{S}$ contained within $20-50 \mathrm{GeV}, 50-100 \mathrm{GeV}$, and $100-150 \mathrm{GeV}$ jets are displayed. These $K_{S}$ have passed the cuts detailed in this section, and the means of the mass distributions are independent of the jet $E_{T}$ and agree with the measured mass [9]. The mass resolutions vary little from $0.6848 \mathrm{E}-02 \mathrm{GeV}$ to $0.7432 \mathrm{E}-02 \mathrm{GeV}$ between the $20-50$ $\mathrm{GeV}$ and the $100-150 \mathrm{GeV}$ cases. This will be better understood once the efficiencies in Chapter 7 are studied. In short, only $20-50 \mathrm{GeV}$ case has higher efficiencies (see Figure 7.16), and this explains why the mass resolution is slightly narrower for the $K_{S}$ inside $20-50 \mathrm{GeV}$ jets.

In summary, the cuts are listed below. 
Table 6.4: The number of events in the Jet20, Jet50, Jet70, and Jet100 samples after each successive cut.

\begin{tabular}{|c|c|c|c|c|c|c|c|c|}
\hline Cuts & $\begin{array}{c}\text { Jet } 20 \\
\text { Events }\end{array}$ & $\%$ & $\begin{array}{c}\text { Jet } 50 \\
\text { Events }\end{array}$ & $\%$ & $\begin{array}{c}\text { Jet } 70 \\
\text { Events }\end{array}$ & $\%$ & $\begin{array}{c}\text { Jet } 100 \\
\text { Events }\end{array}$ & $\%$ \\
\hline Only Cosmic Filter & 541,158 & 100.0 & 320,177 & 100.0 & 307,891 & 100.0 & $1,381,935$ & 100.0 \\
Trigger Cut & 526,158 & 97.2 & 299,986 & 93.7 & 256,559 & 83.3 & $1,381,935$ & 100.0 \\
Badrun & 525,822 & 97.2 & 296,496 & 92.6 & 250,571 & 81.4 & $1,379,960$ & 99.9 \\
$\left|z_{\text {primary }}\right|<60 \mathrm{~cm}$ & 488,177 & 90.2 & 273,674 & 85.5 & 231,468 & 75.2 & $1,272,470$ & 92.1 \\
\hline
\end{tabular}

- $K_{S}$ Cuts

$-K_{S}$ within $\Delta R=0.7$ jet cone

$-z_{j e t}^{r m s}<5.0 \mathrm{~cm}$

$-\left|z_{\text {primary }}-z_{j e t}\right|<5.0 \mathrm{~cm}$

$-\chi_{K S}^{2}<20.0$

$-\left|z_{K S^{-}} z_{\text {primary }}\right|<3.0 \mathrm{~cm}$

- $p_{T}^{\text {tracks from } K S}>500 \mathrm{MeV}$

$-\left|\eta^{K S}\right|<1.0$

- 3D Displacement ${ }^{K S}>1.0 \mathrm{~cm}$

$-\cos \theta_{P D}^{K S}>0.990$

$-1.5<p_{T}^{K S}<10.0 \mathrm{GeV}$ 
Table 6.5: The number of jets in the Jet20 sample after each successive cut for 20-50, 50-100, 100-150 GeV $E_{T}$ ranges.

\begin{tabular}{|c|c|c|c|c|c|c|}
\hline Cuts & $\begin{array}{c}20-50 \\
\mathrm{GeV} \\
\text { Jets }\end{array}$ & $\%$ & $\begin{array}{c}50-100 \\
\mathrm{GeV} \\
\text { Jets }\end{array}$ & $\%$ & $\begin{array}{c}100-150 \\
\mathrm{GeV} \\
\text { Jets }\end{array}$ & $\%$ \\
\hline Cosmic Filter & & & & & & \\
and Trigger Cut & 856,235 & 100.0 & 139,186 & 100.0 & 512 & 100.0 \\
Badrun & 856,209 & 100.0 & 139,047 & 99.9 & 331 & 64.6 \\
$\left|z_{\text {primary }}\right|<60.0 \mathrm{~cm}$ & 794,726 & 92.8 & 130,436 & 93.7 & 249 & 48.6 \\
$\left|\eta_{\text {jet }}^{\text {dettor }}\right|<1.0$ & 418,093 & 48.8 & 72,358 & 52.0 & 142 & 27.7 \\
$z_{\text {jet }}^{\text {jms }}<5.0 \mathrm{~cm}$ & 274,642 & 32.1 & 47,383 & 34.0 & 98 & 19.1 \\
$\left|z_{\text {primary }}-z_{\text {jet }}\right|<5.0 \mathrm{~cm}$ & 245,167 & 28.6 & 42,032 & 30.2 & 86 & 16.8 \\
\hline
\end{tabular}

Table 6.6: The number of jets in the Jet50 sample after each successive cut for $20-50,50-100,100-150 \mathrm{GeV} E_{T}$ ranges.

\begin{tabular}{|c|c|c|c|c|c|c|}
\hline Cuts & $\begin{array}{c}20-50 \\
\mathrm{GeV} \\
\mathrm{Jets}\end{array}$ & $\%$ & $\begin{array}{c}50-100 \\
\mathrm{GeV} \\
\text { Jets }\end{array}$ & $\%$ & $\begin{array}{c}100-150 \\
\mathrm{GeV} \\
\text { Jets }\end{array}$ & $\%$ \\
\hline Cosmic Filter & & & & & & \\
+ Trigger Cut & 215,604 & 100.0 & 453,249 & 100.0 & 70,390 & 100.0 \\
Badrun & 215,364 & 99.9 & 451,176 & 99.5 & 65,195 & 92.6 \\
$\left|z_{\text {primary }}\right|<60.0 \mathrm{~cm}$ & 196,379 & 91.1 & 417,999 & 92.2 & 65,032 & 92.4 \\
$\left|\eta_{\text {jet }}^{\text {dettor }}\right|<1.0$ & 94,230 & 43.7 & 268,263 & 59.2 & 42,647 & 60.6 \\
$z_{\text {jet }}^{\text {rms }}<5.0 \mathrm{~cm}$ & 60,040 & 27.8 & 186,763 & 41.2 & 29,108 & 41.4 \\
$\left|z_{\text {primary }}-z_{\text {jet }}\right|<5.0 \mathrm{~cm}$ & 54,267 & 25.2 & 168,951 & 37.3 & 26,131 & 37.1 \\
\hline
\end{tabular}


Table 6.7: The number of jets in the Jet70 sample after each successive cut for $20-50,50-100,100-150 \mathrm{GeV} E_{T}$ ranges.

\begin{tabular}{|c|c|c|c|c|c|c|}
\hline & $\begin{array}{c}20-50 \\
\mathrm{GeV} \\
\text { Jets }\end{array}$ & $\%$ & $\begin{array}{c}50-100 \\
\mathrm{GeV} \\
\text { Jets }\end{array}$ & $\%$ & $\begin{array}{c}100-150 \\
\mathrm{GeV} \\
\text { Jets }\end{array}$ & $\%$ \\
\hline Cosmic Filter & & & & & & \\
+ Trigger Cut & 147,902 & 100.0 & 247,841 & 100.0 & 257,864 & 100.0 \\
Badrun & 147,424 & 99.7 & 246,535 & 99.5 & 253,878 & 98.5 \\
$\left|z_{\text {primary }}\right|<60.0 \mathrm{~cm}$ & 134,909 & 91.2 & 222,567 & 89.8 & 241,343 & 93.6 \\
$\left|\eta_{\text {jet }}^{\text {detor }}\right|<1.0$ & 67,365 & 45.5 & 140,498 & 56.7 & 180,045 & 69.8 \\
$z_{\text {jet }}^{\text {rms }}<5.0 \mathrm{~cm}$ & 41,700 & 28.2 & 98,285 & 39.7 & 124,148 & 48.1 \\
$\left|z_{\text {primary }} z_{\text {jet }}\right|<5.0 \mathrm{~cm}$ & 38,376 & 25.9 & 90,657 & 36.6 & 113,808 & 44.1 \\
\hline
\end{tabular}

Table 6.8: The number of jets in the Jet100 sample after each successive cut for $20-50,50-100,100-150 \mathrm{GeV} E_{T}$ ranges.

\begin{tabular}{|c|c|c|c|c|c|c|}
\hline Cuts & $\begin{array}{c}20-50 \\
\mathrm{GeV} \\
\text { Jets }\end{array}$ & $\%$ & $\begin{array}{c}50-100 \\
\mathrm{GeV} \\
\text { Jets }\end{array}$ & $\%$ & $\begin{array}{c}100-150 \\
\mathrm{GeV} \\
\text { Jets }\end{array}$ & $\%$ \\
\hline Cosmic Filter & & & & & & \\
+ Trigger Cut & $1,137,327$ & 100.0 & $1,443,838$ & 100.0 & $1,707,897$ & 100.0 \\
Badrun & $1,124,784$ & 98.9 & $1,435,133$ & 99.4 & $1,672,636$ & 97.9 \\
$\left|z_{\text {primarg }}\right|<60.0 \mathrm{~cm}$ & $1,015,976$ & 89.3 & $1,265,212$ & 87.6 & $1,569,682$ & 91.9 \\
$\left|\eta_{\text {jetector }}^{\text {det }}\right|<1.0$ & 511,508 & 45.0 & 743,847 & 51.5 & $1,175,526$ & 68.8 \\
$z_{\text {jet }}^{\text {rns }}<5.0 \mathrm{~cm}$ & 313,346 & 27.6 & 504,708 & 35.0 & 805,874 & 47.2 \\
$\left|z_{\text {primary }}-z_{\text {jet }}\right|<5.0 \mathrm{~cm}$ & 286,431 & 25.2 & 463,542 & 32.1 & 736,875 & 43.1 \\
\hline
\end{tabular}


Table 6.9: The number of $K_{S}$ in the Jet20, Jet50, Jet70, and Jet100 samples after each successive cut.

\begin{tabular}{|c|c|c|c|c|}
\hline Cuts & $\begin{array}{c}\text { Number } \\
\text { of } K_{S} \\
\text { in Jet } 20\end{array}$ & $\begin{array}{c}\text { Number } \\
\text { of } K_{S} \\
\text { in Jet } 50\end{array}$ & $\begin{array}{c}\text { Number } \\
\text { of } K_{S} \\
\text { in Jet } 70\end{array}$ & $\begin{array}{c}\text { Number } \\
\text { of } K_{S} \\
\text { in Jet } 100\end{array}$ \\
\hline $\begin{array}{c}\text { Only Cosmic Filter } \\
\text { + Trigger Cut }\end{array}$ & $143217 \pm 1584$ & $97372 \pm 1394$ & $86619 \pm 1405$ & $594055 \pm 3656$ \\
Badrun & $143167 \pm 1584$ & $97202 \pm 1394$ & $86120 \pm 1400$ & $587459 \pm 3644$ \\
$\left|z_{\text {primary }}\right|<60.0 \mathrm{~cm}$ & $134531 \pm 1540$ & $91150 \pm 1346$ & $80664 \pm 1367$ & $543020 \pm 3507$ \\
$\chi_{K S}^{2}<20.0$ & $113811 \pm 1162$ & $77689 \pm 1056$ & $70211 \pm 1100$ & $472911 \pm 2807$ \\
$\left|z_{K S^{-}} z_{\text {primary }}\right|<3.0 \mathrm{~cm}$ & $110787 \pm 1142$ & $75925 \pm 1041$ & $68575 \pm 1078$ & $460783 \pm 2771$ \\
$p_{T}^{\text {tracks }}>500 \mathrm{MeV}$ & $70522 \pm 778$ & $52332 \pm 796$ & $48592 \pm 854$ & $327799 \pm 2160$ \\
$\left|\eta^{K S}\right|<1.0$ & $61340 \pm 703$ & $46332 \pm 738$ & $43692 \pm 804$ & $293571 \pm 2032$ \\
$3 \mathrm{D} \mathrm{Dis}{ }^{K S}>1.0 \mathrm{~cm}$ & $58175 \pm 544$ & $42340 \pm 572$ & $40397 \pm 624$ & $270102 \pm 1580$ \\
$\cos \theta_{P D}{ }^{K S}>0.990$ & $56651 \pm 497$ & $41476 \pm 535$ & $39590 \pm 581$ & $262582 \pm 1450$ \\
$1.5<p_{T}^{K S}<10 \mathrm{GeV}$ & $47027 \pm 450$ & $34924 \pm 467$ & $32998 \pm 498$ & $221953 \pm 1304$ \\
\hline
\end{tabular}


Table 6.10: The number of $K_{S}$ in $20-50 \mathrm{GeV}$ jets in the Jet20, Jet50, Jet70, and Jet100 samples after each successive cut.

\begin{tabular}{|c|c|c|c|c|}
\hline Cuts & $\begin{array}{c}\text { Number } \\
\text { of } K_{S} \\
\text { in Jet } 20 \\
20-50 \\
\mathrm{GeV} \\
\text { Jets }\end{array}$ & $\begin{array}{c}\text { Number } \\
\text { of } K_{S} \\
\text { in Jet } 50 \\
20-50 \\
\mathrm{GeV} \\
\text { Jets }\end{array}$ & $\begin{array}{c}\text { Number } \\
\text { of } K_{S} \\
\text { in Jet } 70 \\
20-50 \\
\text { GeV } \\
\text { Jets }\end{array}$ & $\begin{array}{c}\text { Number } \\
\text { of } K_{S} \\
\text { in Jet } 100 \\
20-50 \\
\mathrm{GeV} \\
\text { Jets }\end{array}$ \\
\hline Cosmic Filter & & & & \\
\hline & $126 \pm 918$ & $12789 \pm 506$ & $8579 \pm 429$ & $64166 \pm 1121$ \\
\hline Badrun & $122 \pm 918$ & $12792 \pm 506$ & $8561 \pm 426$ & $63845 \pm 1121$ \\
\hline$\left|z_{\text {primary }}\right|<60.0 \mathrm{~cm}$ & $46551 \pm 893$ & $11940 \pm 487$ & $7954 \pm 426$ & $59245 \pm 1082$ \\
\hline$\left|\eta_{\text {jet }}^{\text {detector }}\right|<1.0$ & $41408 \pm 818$ & $10293 \pm 439$ & $7270 \pm 403$ & $52384 \pm 1008$ \\
\hline$z_{j e t}^{r m s}<5.0 \mathrm{~cm}$ & $29756 \pm 650$ & $7087 \pm 346$ & $4918 \pm 300$ & $35870 \pm 795$ \\
\hline$\left|z_{\text {primary }}-z_{\text {jet }}\right|<5.0 \mathrm{~cm}$ & $28401 \pm 627$ & $6940 \pm 335$ & $4778 \pm 292$ & $34826 \pm 765$ \\
\hline$\chi_{K S}^{2}<20.0$ & $27461 \pm 560$ & $6544 \pm 307$ & $4666 \pm 264$ & $33631 \pm 692$ \\
\hline$\left|z_{K S^{-}} z_{\text {primary }}\right|<3.0 \mathrm{~cm}$ & $26710 \pm 550$ & $6431 \pm 304$ & $4594 \pm 260$ & $32916 \pm 683$ \\
\hline$p_{T}^{\text {tracks }}>500 \mathrm{MeV}$ & $21151 \pm 435$ & $5024 \pm 229$ & $3572 \pm 218$ & $26048 \pm 548$ \\
\hline$\left|\eta^{K S}\right|<1.0$ & $20159 \pm 427$ & $4746 \pm 214$ & $3463 \pm 215$ & $24786 \pm 528$ \\
\hline $3 \mathrm{D} \mathrm{Dis}{ }^{K S}>1.0 \mathrm{~cm}$ & $19066 \pm 310$ & $4452 \pm 173$ & $3275 \pm 160$ & $22895 \pm 397$ \\
\hline $\cos \theta_{P D}{ }^{K S}>0.990$ & & $4356 \pm 165$ & $3157 \pm 143$ & $22126 \pm 366$ \\
\hline $1.5<p_{T}^{K S}<10.0 \mathrm{GeV}$ & $16886 \pm 297$ & $3995 \pm 161$ & $2807 \pm 127$ & $20026 \pm 332$ \\
\hline
\end{tabular}


Table 6.11: The number of $K_{S}$ in $50-100 \mathrm{GeV}$ jets in the Jet20, Jet50, Jet70, and Jet100 samples after each successive cut.

\begin{tabular}{|c|c|c|c|c|}
\hline Cuts & $\begin{array}{c}\text { Number } \\
\text { of } K_{S} \\
\text { in Jet } 20 \\
50-100 \\
\text { GeV } \\
\text { Jets }\end{array}$ & $\begin{array}{c}\text { Number } \\
\text { of } K_{S} \\
\text { in Jet } 50 \\
50-100 \\
\text { GeV } \\
\text { Jets }\end{array}$ & $\begin{array}{c}\text { Number } \\
\text { of } K_{S} \\
\text { in Jet } 70 \\
50-100 \\
\text { GeV } \\
\text { Jets }\end{array}$ & $\begin{array}{c}\text { Number } \\
\text { of } K_{S} \\
\text { in Jet } 100 \\
50-100 \\
\text { GeV } \\
\text { Jets }\end{array}$ \\
\hline Cosmic Fi & & & & \\
\hline+ Tri & $9879 \pm 524$ & $35254 \pm 912$ & $19437 \pm 729$ & $108992 \pm 176$ \\
\hline Badrun & $79 \pm 524$ & $35249 \pm$ & $19423 \pm$ & $108910 \pm 1766$ \\
\hline$\left|z_{\text {primary }}\right|<60.0 \mathrm{~cm}$ & $9453 \pm 517$ & $33119 \pm 898$ & $18070 \pm 737$ & $98859 \pm 1722$ \\
\hline$\left|\eta_{\text {jet }}^{\text {detector }}\right|<1.0$ & $8435 \pm 487$ & $30683 \pm 848$ & $16533 \pm 710$ & $87980 \pm 1568$ \\
\hline$z_{\text {jet }}^{r m s}<5.0 \mathrm{~cm}$ & $5734 \pm 357$ & $23070 \pm 686$ & $12468 \pm 599$ & $65563 \pm 1285$ \\
\hline$\left|z_{\text {primary }}-z_{\text {jet }}\right|<5.0 \mathrm{~cm}$ & $5466 \pm 339$ & $22252 \pm 663$ & $12100 \pm 585$ & $63912 \pm 1250$ \\
\hline$\chi_{K S}^{2}<20.0$ & $5236 \pm 304$ & $21341 \pm 603$ & $11525 \pm 534$ & $61481 \pm 1121$ \\
\hline$\left|z_{K S^{-}} z_{\text {primary }}\right|<3.0 \mathrm{~cm}$ & $5171 \pm 296$ & $20862 \pm 597$ & $11262 \pm 524$ & $59989 \pm 1095$ \\
\hline$p_{T}^{\text {tracks }}>500 \mathrm{MeV}$ & $4131 \pm 236$ & $16938+518$ & $9154 \pm 443$ & $49206 \pm 933$ \\
\hline$\left|\eta^{K S}\right|<1.0$ & $3937 \pm 227$ & $16302 \pm 512$ & $8661 \pm 424$ & $46652 \pm 921$ \\
\hline $3 \mathrm{D} \mathrm{Dis}{ }^{K S}>1.0 \mathrm{~cm}$ & $3670 \pm 178$ & $14722 \pm 400$ & $8161 \pm 330$ & $42954 \pm 711$ \\
\hline $\cos \theta_{P D}{ }^{K S}>0.990$ & $3566 \pm 173$ & $14316 \pm 374$ & $8089 \pm 317$ & $42220 \pm 676$ \\
\hline $1.5<p_{T}^{K S}<10.0 \mathrm{GeV}$ & $3038 \pm 150$ & $12388 \pm 323$ & $6937 \pm 269$ & $36961 \pm 593$ \\
\hline
\end{tabular}


Table 6.12: The number of $K_{S}$ in $100-150 \mathrm{GeV}$ jets in the Jet20, Jet50, Jet70, and Jet100 samples after each successive cut.

\begin{tabular}{|c|c|c|c|c|}
\hline Cuts & $\begin{array}{c}\text { Number } \\
\text { of } K_{S} \\
\text { in Jet } 20 \\
100-150 \\
\text { GeV } \\
\text { Jets }\end{array}$ & $\begin{array}{c}\text { Number } \\
\text { of } K_{S} \\
\text { in Jet } 50 \\
100-150 \\
\text { GeV } \\
\text { Jets }\end{array}$ & $\begin{array}{c}\text { Number } \\
\text { of } K_{S} \\
\text { in Jet } 70 \\
100-150 \\
\text { GeV } \\
\text { Jets }\end{array}$ & $\begin{array}{c}\text { Number } \\
\text { of } K_{S} \\
\text { in Jet } 100 \\
100-150 \\
\text { GeV } \\
\text { Jets }\end{array}$ \\
\hline Cosm & & & & \\
\hline & $N A$ & $5421 \pm 390$ & $23159 \pm 906$ & $148828=$ \\
\hline $\mathrm{Bad}$ & $N A$ & $19 \pm 392$ & 2315 & 14843 \\
\hline $\mid z_{\text {primary }}$ & $N A$ & $11 \pm 385$ & $22135 \pm 881$ & 140523 \\
\hline 78 & $N A$ & $54 \pm 390$ & 20955 & 131467 \\
\hline & $N A$ & $3597 \pm 390$ & $16021 \pm 734$ & 97180 \\
\hline$\left|z_{\text {primary }}-z_{\text {jet }}\right|<5.0 \mathrm{~cm}$ & $N A$ & $3561 \pm 437$ & $15442 \pm 720$ & $94075 \pm 1662$ \\
\hline$\chi_{K S}^{2}<20.0$ & $N A$ & $3377 \pm 332$ & $15007 \pm 646$ & $90778 \pm 1506$ \\
\hline$\left|z_{K S^{-}} z_{\text {primary }}\right|<3.0 \mathrm{~cm}$ & $N A$ & $3309 \pm 314$ & $14594 \pm 602$ & $88699 \pm 1477$ \\
\hline & $N 4$ & $2551 \pm 269$ & $11540 \pm 523$ & $72328 \pm 1267$ \\
\hline 1 & $N A$ & $2525 \pm 262$ & $11205 \pm 519$ & $69816 \pm 1235$ \\
\hline $3 \mathrm{D} \mathrm{Dis}{ }^{K S}>1.0 \mathrm{~cm}$ & $N A$ & $2293 \pm 188$ & $9977 \pm 392$ & $63826 \pm 962$ \\
\hline & $N A$ & $2337 \pm 186$ & $9798 \pm 366$ & $62173 \pm 902$ \\
\hline $1.5<p_{T}^{K S}<10.0 \mathrm{GeV}$ & $N A$ & $1973 \pm 131$ & $8300 \pm 305$ & $53398 \pm 760$ \\
\hline
\end{tabular}



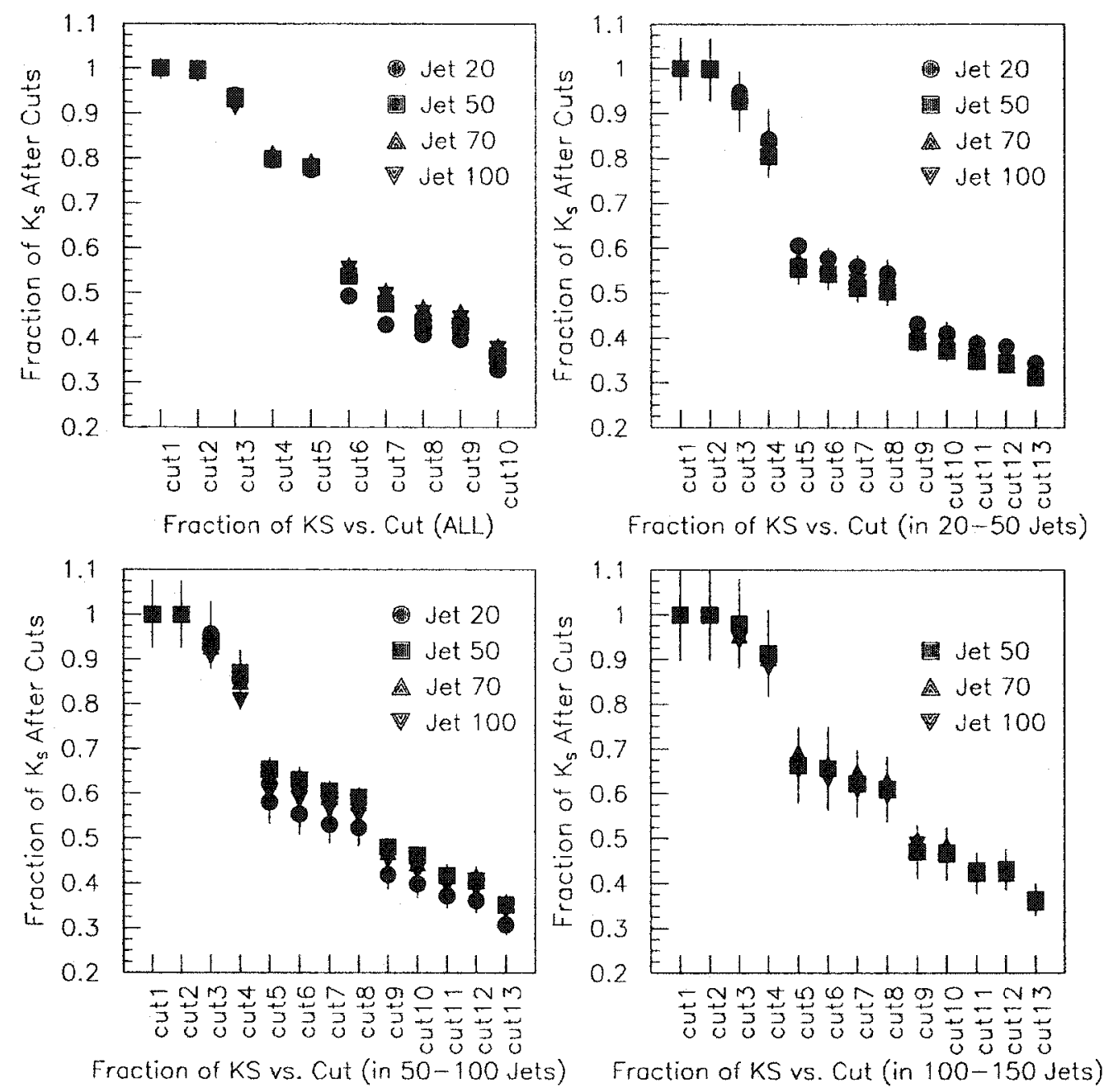

Figure 6.1: The fraction of the $K_{S}$ left after each cut in each jet sample. In the upper left corner, the fraction of the $K_{S}$ left after every cut for all $K_{S}$ is plotted. In the upper right corner, the fraction of the $K_{S}$ left after every cut for all $K_{S}$ inside $20-50 \mathrm{GeV}$ jets is plotted. In the lower left corner, the fraction of the $K_{S}$ left after every cut for all $K_{S}$ inside $50-100 \mathrm{GeV}$ jets is plotted. In the lower right corner, the fraction of the $K_{S}$ left after every cut for all $K_{S}$ inside $100-150 \mathrm{GeV}$ jets is plotted. The cut labels are defined in the following manner: cut $1=$ Cosmic Filter + Trigger Cut, cut $2=$ Badrun, cut $3=\left|z_{\text {primary }}\right|<60.0 \mathrm{~cm}$, cut $4=\eta_{\text {jet }}^{\text {detector }}<1.0$, cut $5=z_{j e t}^{r m s}<5.0 \mathrm{~cm}$, cut $6=\left|z_{\text {primary }}-z_{\text {jet }}\right|<5.0 \mathrm{~cm}$, cut $7=$ $\chi_{K S}^{2}<20.0$, cut8 $=\left|z_{K S^{-}} z_{\text {primary }}\right|<3.0 \mathrm{~cm}$, cut $9=p_{T}^{\text {tracks from } K S}>500 \mathrm{MeV}$, cut 10 $=\left|\eta^{K S}\right|<1.0$, cut11 $=3 \mathrm{D}$ Displacement ${ }^{K S}>1.0 \mathrm{~cm}$, cut12 $=\cos \theta_{P D}{ }^{K S}<0.990$, and cut13 $=1.5 \mathrm{GeV}<p_{T}^{K S}<10.0 \mathrm{GeV}$. 

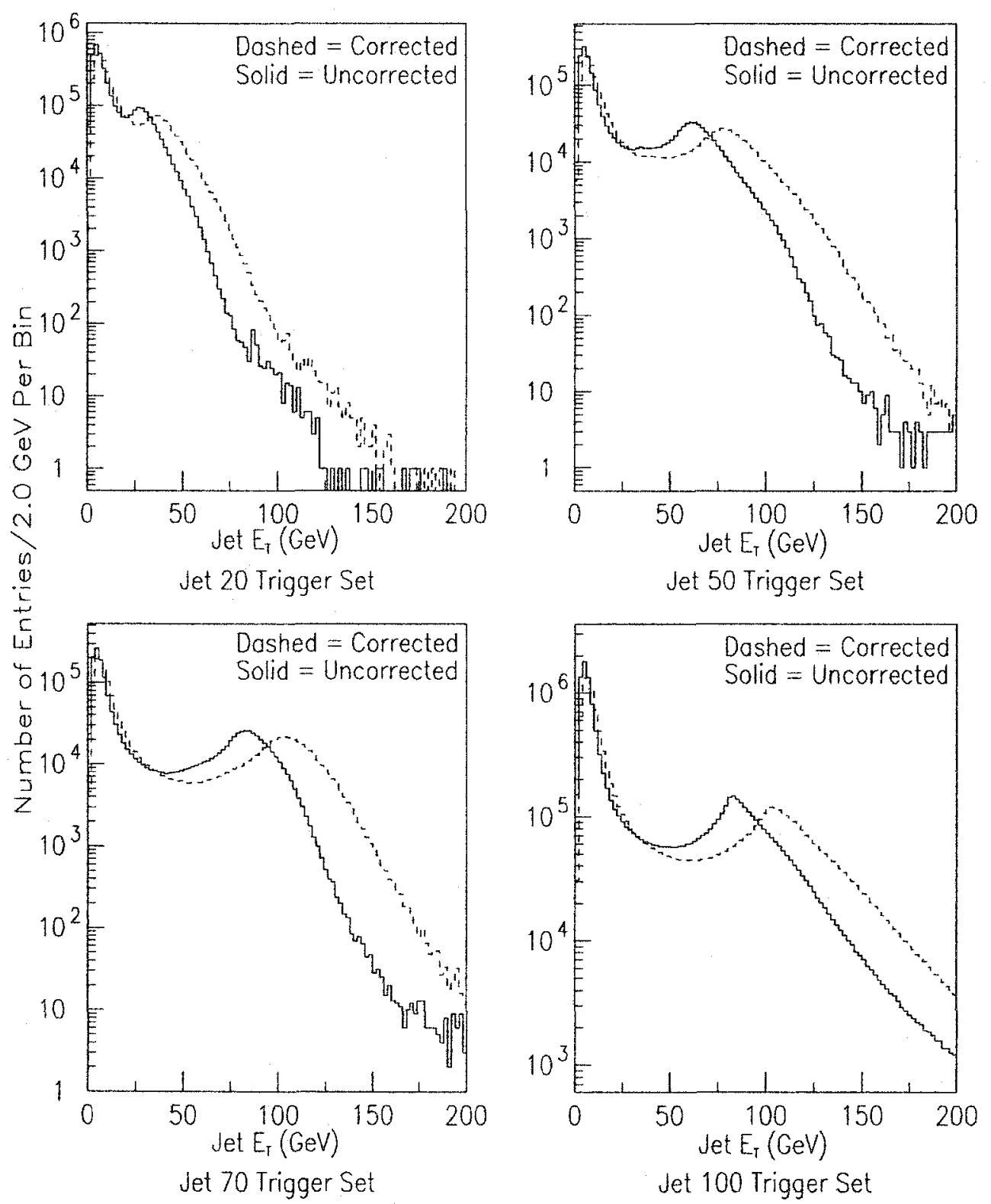

Figure 6.2: The jet $E_{T}$ distribution for the Jet20, Jet50, Jet70, and Jet100 sets. The solid line is the uncorrected jet energy distribution, and the dashed line is the corrected jet energy distribution. 


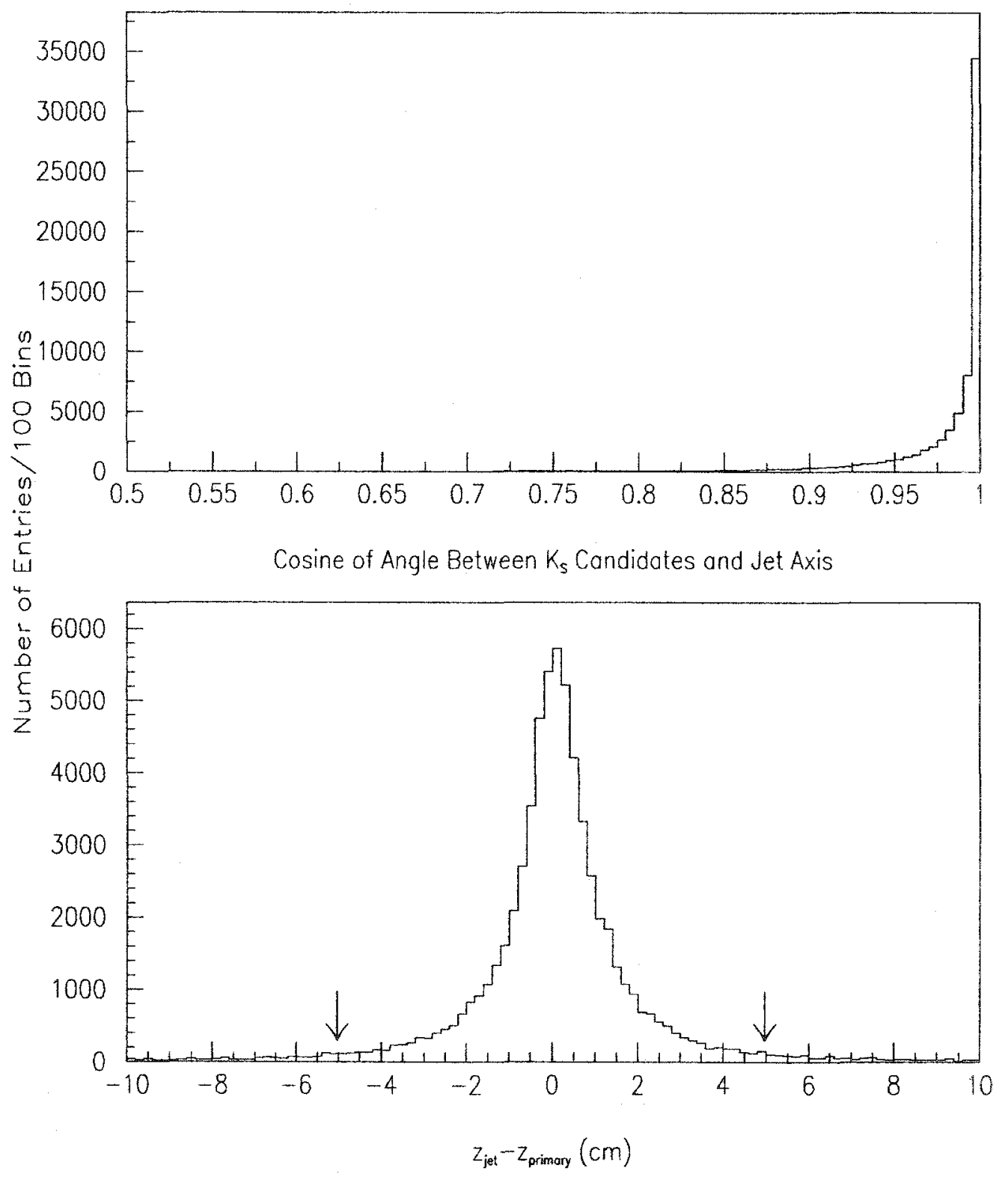

Figure 6.3: In the upper figure, the cosine of the angle between the $K_{S}$ and the jet axis is plotted for $K_{S}$ inside 0.7 cone jets in Jet 50 sample. In the lower figure, the difference between the jet vertex and primary vertex is plotted for the Jet 50 sample. 


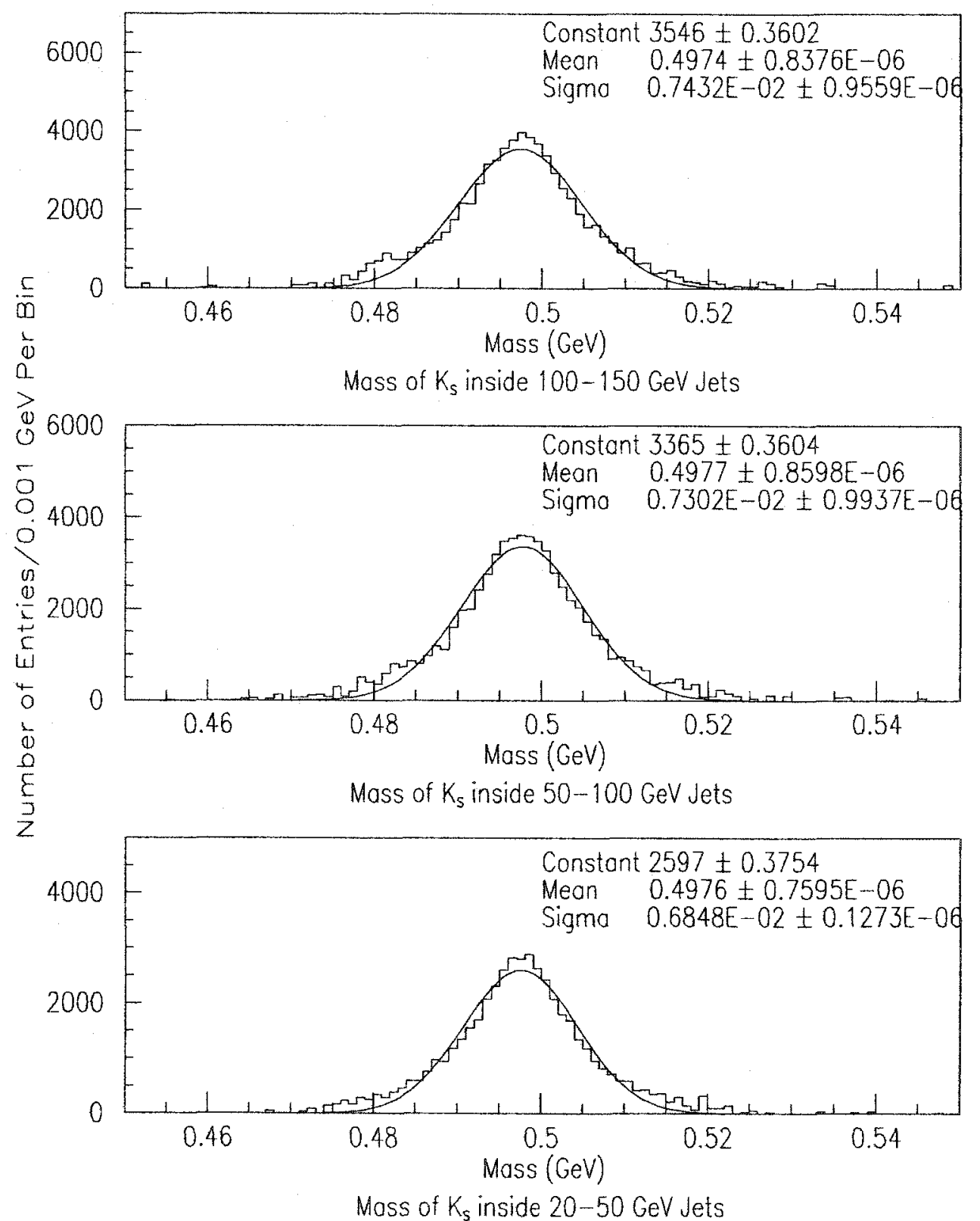

Figure 6.4: The mass distribution of $K_{S}$ candidates after background subtraction after cuts. These particles are found inside $100-150 \mathrm{GeV}$ jets (top), $50-100 \mathrm{GeV}$ jets (middle), and greater than 20-50 GeV Jets (bottom). 


\section{Chapter 7}

\section{Jets: Efficiencies From Track Embedding}

There are many similarities and differences between track embedding in the Minimum Bias and jet samples. Just as in the case for $K_{S}$ in the Minimum Bias data, not all $K_{S}$ are found inside jets in the jet sample. Like the track-embedding $K_{S}$ in the Minimum Bias sample, the efficiency of finding $K_{S}$ in the CDF detector is strongly correlated to the $p_{T}$ of the $K_{S}$. Again, once the $K_{S}$ have been extracted from the data, these efficiency curves are used to correct for the inefficiency of the CTC. However, correcting for $K_{S}$ in jets introduces additional complications than in the Minimum Bias case that must be taken into account.

Since the goal is to study $K_{S}$ production in jets, $K_{S}$ can no longer be placed with random $\eta$ and $\phi$ in the jet data as is done in the Minimum Bias sample, but rather $K_{S}$ must be placed inside 0.7 jet cones. Furthermore, $K_{S}$ are not put in jets with random $\eta$ and $\phi$ anywhere inside 0.7 jet cones. Instead, the $K_{S}$ are deposited in jets similar to other particles. Also, $K_{S}$ are not embedded in any jet in the jet data sample. $K_{S}$ have to be embedded in jets that have jet vertices relatively close to the primary vertices so that the secondary vertex algorithm can find them (i.e. $K_{S}$ would fail the pointing back criteria). Additional factors include depositing $K_{S}$ inside jets and finding them, but the $K_{S}$ are reconstructed outside the jet 
cones would be considered lost. Moreover, $K_{S}$ efficiency is now dependent on jet properties such as jet $E_{T}$. Another important factor is that $K_{S}$ must be embedded in a jet sample having luminosity along with jet properties similar to those of the data.

In this chapter, a review of track-embedding will be reiterated, followed by a detailed track-embedding procedure that addresses some issues described above. Then, comparisons between $K_{S}$ and background distributions will be made as well as comparisons between $K_{S}$ in data and $K_{S}$ in track-embedded Monte Carlo. $K_{S}$ in jet efficiencies as a function of luminosity, $\eta_{j e t}, \phi_{p h i},\left|z_{\text {primary }}-z_{j e t}\right|$, and the number of tracks per jet will also be presented along with the actual efficiency curves needed to correct the $K_{S}$ inside jets in the jet data. Finally, since $K_{S}$ production will be compared to charged particle production, the calculation of single track efficiency in jets curves will be described in order to later correct the single track $p_{T}$ spectrum in jets.

\subsection{Track-embedding Reiterated}

By depositing hits of Monte Carlo $K_{S}$ into the CTC data and rerunning reconstruction, the efficiency of finding $K_{S}$ in data can be determined. The efficiency of CDF detector of finding $K_{S}$ is strongly correlated to the $p_{T}$. Once $K_{S} p_{T}$ curves have been extracted from data, these efficiency curves as a function of $p_{T}$ can be used to correct for the inefficiency of the CTC.

About 10,000 actual jet events are employed for the track embedding study. The sample is checked to have a similar luminosity distribution to actual jet data as well as pass all criteria specified for jet events. A simple tally of good track-embedding events is kept (events in which one $K_{S}$ is embedded into one jet in one event). Jet 
events just pass the QCDB triggers (one trigger before the triggers used to produce the JET20, JET50, JET70, and JET100 sets) because later triggers drop the banks needed to embed hits. Since events pass jet triggers, each event is more likely to have high track multiplicity and many more CTC hits than Minimum Bias events. In Figure 7.2, a transverse view of the CTC is given for a typical jet event selected prior to track embedding. The dots are the actual hits in the CTC, and the fitting of the hits yields the tracks given by solid lines. Only charged particles create hits in the CTC, and only charged particles can be seen directly with CTC. $K_{S}$ being all neutral would themselves be invisible. Only the daughter pions can be seen directly. Also, the greater the $p_{T}$ of a track, the less visible the curvature.

In Figure 7.3, the event in Figure 7.2 has had $3.0 \mathrm{GeV} K_{S}$ embedded into a $136.9 \mathrm{GeV}$ jet. If you compare the 1:00 position of Figure 7.2 and Figure 7.3, you should count 10 tracks in Figure 7.2 and 12 tracks in Figure 7.3. The additional 2 tracks are the embedded $3.0 \mathrm{GeV} K_{S}$. The high $p_{T}$ pion is new straight track, and the low $p_{T}$ pion track is the new curved track. The tracks of the pions intersect at the approximately the same point the $K_{S}$ decays forming a "V". However, some events in which track embedding is attempted does not successfully reveal $K_{S}$. A $K_{S}$, if it has too much $p_{T}$, can decay outside the CTC; a $K_{S}$ may also slip through a crack so that it is never found. The daughter particles can also escape the detection of CTC, especially if the $p_{T}$ of the individual tracks are too soft. This is quite often the case with pions and explains most of the loss of efficiency for $K_{S}$. The efficiency is given as the number of a $K_{S}$ in jets found divided by the number of the events that have had $K_{S}$ track-embedded into them.

Since the embedding procedure in jets is complex and will be discussed in greater detail in the next section, for now the daughter particles of $K_{S}$ are "magically", 
details saved for the next section, embedded inside jets at the primary vertex as hits in the CTC. "Magically", kinematic and vertice information of daughter particles of $K_{S}$ are saved in text files after $K_{S}$ are produced and decayed in FAKEEVENT and CDFSIM, and the daughters of the $K_{S}$ are then embedded inside jets such that $K_{S}$ a distribution similar to other particles. The distributions of jets incorporated for track-embedding is similar to the distributions of the other jets in the jet data, and the embedding jet events are liken to the events found in jet events. The information in the text file is adjusted in order to embedded the daughters of the $K_{S}$ at the primary vertices close to the jet vertices, and 4-vector and vertice information from the daughters of the $K_{S}$ are converted to track parameters, and track parameters are converted to hits in the CTC. Once hits are added to the data, reconstruction is rerun to produce a new list of tracks from which code can then be applied to discover whether $K_{S}$ in a specified embedded jet can be found.

In determining $K_{S}$ inside jets in the track-embedded data, identical selection criteria are utilized as described in Chapter 6 with the exception of using a reconstructed $K_{S} \quad p_{T}$ window cut. As before in the Minimum Bias track-embedding, since the $p_{T}$ of track-embedded $K_{S}$ is known, the information is necessary in order to separate the $K_{S}$ candidate from $K_{S}$ already present in the data set. Table 7.2 has the ranges of the $K_{S}$ shown along with the $p_{T}$ window for each range. In Figure $7.4,1.6 \mathrm{GeV}$ and $6.0 \mathrm{GeV}$ track-embedded $K_{S}$ candidates are displayed. The solid line represents the $K_{S}$ that fail the $p_{T}$ window cut and may be $K_{S}$ already present in the data, and the dashed line shows the embedded $K_{S}$ that pass the $p_{T}$ window cut. $K_{S}$ already inside the embedding data may or may not be within jets, and these $K_{S}$ give rise to solid line $K_{S}$ mass peak. For $1.6 \mathrm{GeV} K_{S}$, the $p_{T}$ window is $30 \mathrm{MeV}$, and for the $6.0 \mathrm{GeV} K_{S}$, the $p_{T}$ window is $0.5 \mathrm{GeV}$. On the 
bottom figures of Figure 7.4, the fitted background subtracted mass plots of $K_{S}$ passing the $p_{T}$ window cut are plotted. Figure 7.4 shows that the mass resolution of $K_{S}$ in jets increases from approximately $0.006 \mathrm{GeV}$ for $1.6 \mathrm{GeV}$ embedded $K_{S}$ to approximately $0.008 \mathrm{GeV}$ for $6.0 \mathrm{GeV}$ embedded $K_{S}$. Figure 7.5 contains the $p_{T}$ of the $K_{S}$ along with arrows indicating the range of the $p_{T}$ window. The bottom figures in Figure 7.5 reveals the background subtracted fitted $p_{T}$ of $K_{S}$ that are within the $p_{T}$ window. In Figure 7.5 (below), the resolution of the $p_{T}$ increases from approximately $0.006 \mathrm{GeV}$ for $1.6 \mathrm{GeV}$ embedded $K_{S}$ to about $0.07 \mathrm{GeV}$ for $6.0 \mathrm{GeV}$ embedded $K_{S}$.

The number of $K_{S}$ in jets are calculated at a range of $p_{T}$ intervals. In top of Figure 7.4, there is a small mass peak (dashed line) that passes the $p_{T}$ window cuts as well as the event and jet cuts. Once the background has been subtracted from this peak, the resulting mass peaks (fitted plots on the bottom of Figure 7.4) can be fitted and the number of $K_{S}$ can be summed from 0.48 to $0.52 \mathrm{GeV}$. The efficiency is given by the number of $K_{S}$ found within jets divided by the number of $K_{S}$ embedded inside jets. The points are then plotted against $p_{T}$ using fitting errors to produce plots in Figures 7.16-7.18. The upper portion of each figure consists of each point as a function of $p_{T}$, and lower portions of Figure 7.16-7.18 are each fitted for two different $p_{T}$ ranges. These curves are needed to correct the $p_{T}$ spectra of the $K_{S}$ in the data. Note that in Minimum Bias case, there is only a single efficiency curve, and now there are three efficiency curves, one for each jet $E_{T}$ range. This is done because $K_{S}$ in jet efficiency is also a function of jet $E_{T}$. The next section will describe the track-embedding procedure in much more detail. 


\subsection{Track-embedding Jet Procedure}

Step 1) Obtaining the $\Delta R_{\text {jet-track }}$ distribution of tracks in jets.

The $K_{S}$ will be placed inside jets in a manner similar to all other particles since there is not any reason that $K_{S}$ should be distributed differently. The distribution of tracks as function of $\Delta R_{\text {jet-track }}$ is defined by:

$$
\Delta R_{\text {jet-track }}=\sqrt{\left(\eta_{j e t}-\eta_{t r a c k}\right)^{2}+\left(\phi_{j e t}-\phi_{\text {track }}\right)^{2}}
$$

Also, only the shape of the distribution is of interest, and this changes slightly for efficiency corrections (CTC track efficiency is high), track $p_{T}$ cuts, $\eta$ cuts, number of events, etc. Many of these cuts are studied as to what effect each has on the shapes of the $\Delta R_{\text {jet-track }}$ distribution, and one variable that has a significant effect is that of the jet $E_{T}$. The $\Delta R_{j e t-t r a c k}$ distribution can be a strong function of jet $E_{T}$, especially at low jet $E_{T}$.

The entire jet set is ran through with the following cuts and is the first step in producing the distributions:

- Track cuts for the production of $\Delta R_{\text {jet-track }}$ distributions.

- Event Cuts

$-\left|z_{\text {primary }}-z_{j e t}\right|<5.0 \mathrm{~cm}$

$-\left|z_{\text {primary }}-z_{\text {track }}\right|<5.0 \mathrm{~cm}$

- Track is associated with the nearest jet within $\Delta R_{\text {jet-track }}<1.0$

- Booked according to Jet $E_{T}$ Ranges

There are over $25 \Delta R_{\text {jet-track }}$ distributions corresponding to different jet $E_{T}$ ranges, and each distribution is subtracted by a flat constant function which yields 
0.0 at $\Delta R_{\text {jet-track }}=0.7$. These are the distributions implemented in the track embedding. In Figure 7.1, $\Delta R_{\text {jet-track }}$ are grouped into just 3 energy ranges, 20-50 $\mathrm{GeV}, 50-100 \mathrm{GeV}$, and $100-150 \mathrm{GeV}$, and are normalized to unit area. As jet $E_{T}$ increases, the shape of the $\Delta R_{\text {jet-track }}$ distribution becomes broader as the peak of the distribution moves to larger values of $\Delta R_{\text {jet-track}}$. This is not a particularly large efficiency effect.

Step 2) Create text files of 4-vector and vertice data of $K_{S}$ daughters. Create the text files of 4-vectors and primary and secondary vertice information of daughters from $K_{S}$ at random $\eta,|\eta|<2.0$, and random $\phi, 0 \leq \phi<2 \pi$. These are the exact text files incorporated into the Minimum Bias track-embedding produced with FAKEEVENT and CDFSIM.

\section{Step 3) Select an embedding data sample.}

The embedding sample consists of events from a data set that has CTC hit banks. Unfortunately, the CTC hit banks are not found in the JET20, JET50, JET70, and JET100 sets (they are dropped as these sets are created). Hence, the parent set (QJTB) for the jet trigger samples is needed for the track embedding. In addition, not any subset of QJTB would do, but the sample has to include events with a instantaneous luminosity distribution similar to that of the jet data set. Otherwise, luminosity efficiency corrections would be needed. The events must satisfy the criteria of cosmic filter (all other event cuts will be applied later). The event also must have at least one of the two highest energy jets with a primary vertex within $5.0 \mathrm{~cm}$ of the jet vertex. This last requirement is to optimize the track embedding process (virtually all jet vertices that are within $5.0 \mathrm{~cm}$ of the primary vertex are from the 2 highest $E_{T}$ jets).

Step 4) Select jets for track-embedding. 
First, jets must have $\left|\eta_{\text {jet }}\right|<2.0$ before being considered because the CTC coverage is approximately $|\eta|<1.0$. Since for every event, there is only one $K_{S}$ embedded into only one jet, some caution must be exercised to avoid having all $K_{S}$ embedded into jets of a particular energy range. In particular, there is an algorithm and "cap" designed to limit the number of events with a certain jet energy. For example, if there are 10000 jets between $100-150 \mathrm{GeV}$ and 500 jets between $50-100 \mathrm{GeV}$, the program will keep the 500 jets between $50-100 \mathrm{GeV}$ and up to 2000 jets between $100-150 \mathrm{GeV}$. The jet $E_{T}$ are corrected prior to this selection process; all other jet cuts are applied later. It is important to clarify that the properties of the jet taken for embedding are saved for later and are denoted by $\phi_{\text {embed }}, \eta_{\text {embed }}$, and jet $E_{T}^{e m b e d}$.

Step 5) Selecting an embedding direction in a jet for track-embedding. Once an embedding jet is selected, applying Monte Carlo techniques to the $\Delta R_{\text {jet-track }}$ distribution for the particular jet $E_{T}$ sets an embedding direction with a given $\phi_{\text {direction }}$ and $\eta_{\text {direction }}$ with respect to $\phi_{\text {embed }}$ and $\eta_{\text {embed }}$ of the selected jet where the $K_{S}$ will be embedded. This is the embedding direction is where the $K_{S}$ should be placed.

Step 6) Selecting a $K_{S}$ closest to the embedding direction in $\Delta R_{K S \text {-direction }}$. The text file is ran through until a $K_{S}$ closest to the embedding direction is found. The text file is used over and over again without $K_{S}$ being removed from the list. The "randomness" of the $\phi_{\text {direction }}$ and $\eta_{\text {direction }}$ ensures the randomness of the $\phi$


and $\eta_{\text {embed }}$, and the $\eta_{\text {embed }}$ is not a perfectly flat distribution between $\left|\eta_{\text {embed }}\right|<2.0$. However, the $\eta_{\text {embed }}$ are distributed enough to result in a similar $\eta_{K S}$ spectrum to 


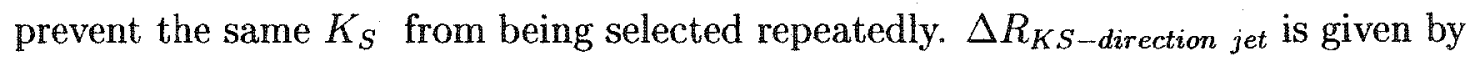

$$
\Delta R_{K S-\text { direction }}=\sqrt{\left(\eta_{K S}-\eta_{\text {direction }}\right)^{2}+\left(\phi_{K S}-\phi_{\text {direction }}\right)^{2}}
$$

$\Delta R_{K S-\text { direction }}$ is mostly less than 0.05 , and the distribution peaks at 0.025 . Hence, the embedding of the $K_{S}$ is not precisely at the embedding direction but about $0.025 \Delta R_{K S-\text { direction }}$ from it.

\section{Step 7) Smear the primary vertex.}

Now that the identity of the $K_{S}$ to be embedded is known, the $K_{S}$ must be embedded using the vertex and 4-vector information from the text file. In particular, just like the Minimum Bias case, the $K_{S}$ must be embedded at the primary with the $\mathrm{x}$ and $\mathrm{y}$ components of the vertex set to 0.0 . Unlike the Minimum Bias case, this time the primary vertex will be smeared by a Gaussian having the sigma given by the distribution of the measurement error of the primary vertex (around $0.25 \mathrm{~cm}$ ). This has a negligible effect on the overall efficiencies and only slightly widens the width of the $K_{S}$. The vertex smearing can be removed from the jet track embedding as well as be added to the Minimum Bias track embedding, both without much of an effect. By virtue of selecting primary vertices from events, the primary vertex distribution of the embedding sample is similar to the primary vertex distribution of the data.

\section{Step 8) Embed $K_{S}$ just as in Minimum Bias track embedding.}

The $K_{S}$ vertice and 4-vector information is converted into hits into the CTC. First, the vertice information from the text file needs to be translated so that its origin is identical to that of the smeared primary. Then, the 4-vector information is converted into the 5 track parameters. After these steps, hits are embedded into the CTC using CTADDH. All embedded jet variables are kept at this stage as well and 
will be denoted by either the superscript or the subscript "embed". Reconstruction is reran to yield a new data set with $K_{S}$ embedded into it.

\section{Step 9) Calculate Efficiencies with new track-embedded data set.}

It is at this stage that efficiencies are calculated. The efficiency consists of a numerator (number of $K_{S}$ in jets after cuts) divided by a denominator (number of events passing certain embedding criteria). In all cases, there is a unique track embedded jet in a unique track embedded event, so in the denominator the phrase "track embedded event" is interchangeable with "track embedded jet". To be consistent, the phrase "track embedded event" will be used to remain consistent with the Minimum Bias discussion.

The number of events must pass the following cuts to be counted as a track embedding event (Denominator):

- Track-embedding Event Cuts

$$
\begin{aligned}
& \text { - Event Cuts } \\
& -\left|z_{\text {primary }}-z_{\text {embedded jet }}\right|<5.0 \mathrm{~cm} \\
& -z_{\text {embedded jet }}^{r m s}<5.0 \mathrm{~cm} \\
& -\left|\eta_{\text {embedded jet }}^{\text {detector }}\right|<1.0 \\
& -20 \mathrm{GeV}<\mathrm{Jet} E_{T}<50 \mathrm{GeV}, 50 \mathrm{GeV}<\operatorname{Jet} E_{T}<100 \mathrm{GeV} \text {, or } \\
& \\
& 100 \mathrm{GeV}<\mathrm{Jet}_{T}<150 \mathrm{GeV}
\end{aligned}
$$

The event cuts are identical to the data event cuts. The $\left|z_{\text {primary }}-z_{\text {embedded jet }}\right|<5.0$ cm criteria ensures that $K_{S}$ are reasonable close to the primary vertex from where the search for $K_{S}$ will begin and that $K_{S}$ are within jet cones at approximately the same z-position as the jet. The $z_{\text {embedded jet }}^{\text {rms }}<5.0 \mathrm{~cm}$ criteria checks to see that 
$z_{\text {embedded jet }}$ is carefully measured. For example, a $z_{\text {embedded jet }}$ having $\mid z_{\text {primary }}{ }^{-}$ $z_{\text {embedded jet }} \mid<0.1 \mathrm{~cm}$ having $z_{\text {embedded jet }}^{\text {rms }}>20.0 \mathrm{~cm}$ should not be counted. The $\left|\eta_{\text {embedded jet }}^{\text {detect }}\right|<1.0$ should keep the jets within the CTC coverage. Finally, a cut is made on the jet $E_{T}$ to allow for three separate efficiency vs. $p_{T}^{K S}$ curves, one for each jet $E_{T}$ range: $20-50 \mathrm{GeV}, 50-100 \mathrm{GeV}$, and $100-150 \mathrm{GeV}$ jets. The values are calculated for each embedded $p_{T}^{K S}$ point, and this number is taken as the denominator in calculation of the efficiency at each embedded $p_{T}^{K S}$ point.

The number of $K_{S}$ must pass the following cuts to be counted as a track embedding event (Numerator):

- Number of $K_{S}$ in jets (Numerator)

- All Cuts Listed in the Above Table (Denominator)

- $K_{S}$ must be inside the embedded jet.

- $K_{S}$ must pass the $p_{T}$ window cut given by Table 7.1.

$-\chi_{K S}^{2}<20.0$

$-\left|z_{K S}-z_{\text {primary }}\right|<3.0 \mathrm{~cm}$

$-p_{T}^{\text {tracks from } K S}>500 \mathrm{MeV}$

$-\left|\eta^{K S}\right|<1.0$

- 3D Displacement ${ }^{K S}>1.0 \mathrm{~cm}$

$-\cos \theta_{P D}^{K S}>0.990$

Out of the jets that pass all of the event and jet cuts, the number of embedded $K_{S}$ found the embedded jets determines the numerator of the efficiency calculation. To ensure that a given jet is the embedded jet, a strict $\left|E_{T}^{\text {jet }}-E_{T}^{\text {embedded jet }}\right|<0.001$ $\mathrm{GeV}$ is necessary along with event and jet cuts. As before in the Minimum Bias set, 
a $p_{T}$ window cut which varies according $p_{T}$ of the embedded $K_{S}$ (see Table 7.1) is applied to reduce the contamination of $K_{S}$ already in the data set. Only those $K_{S}$ within the $p_{T}$ window cut along with all other cuts are tallied. By dividing the numerator term by the denominator term, the efficiency of $K_{S}$ at a particular $p_{T}$ value can be determined.

In addition, all Minimum Bias $K_{S}$ cuts appear again with the same cut values with the exception of the 2D pointing vector cut (decreases from 0.995 to 0.990 ) and the $p_{T}^{\text {tracks }}$ from KS (increases from $300 \mathrm{MeV}$ to $0.5 \mathrm{GeV}$ ). The increase in the $p_{T}^{\text {tracks from } K S}$ is due to the overall increase in $p_{T}$ of the $K_{S}$ in jets as well as keeping the individual $p_{T}$ cut in the region where the CTC track efficiency is more reliable. The change in the $2 \mathrm{D}$ pointing vector takes into account the widening of the distribution between the minimum bias and jet samples, and the differences between the two cut values is not significant.

All cut variables for both $1.6 \mathrm{GeV}$ and $6.0 \mathrm{GeV} K_{S}$ in jets are shown in Figures 7.6-7.9. The dashed line indicates candidates satisfying the $p_{T}$ window cut, and the solid line denotes candidates that fail the $p_{T}$ window cut. The arrows point to the actual cut values. In addition to the cuts seen before in the Minimum Bias scenario, there are the $z_{\text {primary }}-z_{j e t}$ and $z_{j e t}^{r m s}$ distributions (Figure 7.6).

\subsection{Efficiency Dependencies}

In this section, the $K_{S}$ inside jets efficiency as a function of $E_{T}^{j e t}, \eta_{j e t}, \phi_{j e t}$, luminosity, $\left|z_{K S^{-}} z_{\text {primary }}\right|$, and jet track multiplicity (the number of tracks within a jet) will be discussed. For each of these variables, the $K_{S}$ in jets are subdivided by whatever property that is being studied after the $K_{S}$ cuts have been applied. To illustrate, for the generation of the efficiency vs. $\phi_{j e t}$ plot (see figure 7.12), the $K_{S}$ in jets 
(numerator) are calculated just as before with the exception that the $K_{S}$ in jets is further subdivided into $K_{S}$ in jets with particular ranges of $\phi_{j e t}$. Similarly, the number of embedded events (denominator) are determined but instead the events are subdivided into events having jets with particular $\phi_{j e t}$ values. In this case, the five $\phi_{j e t}$ ranges are $0-1,1-2,2-3,3-4$, and $5-2 \pi$.

If there were not an jet $E_{T}$ dependence, there would be $5 \phi_{\text {jet }}$ efficiencies for every $K_{S}$ embedded $p_{T}$. However, there is a known $K_{S}$ efficiency dependence on jet $E_{T}$, so the $K_{S}$ in jets (numerator) and the events for each $\dot{\phi}_{j e t}$ range is further subdivided in to three jet energy ranges, $20-50 \mathrm{GeV}, 50-100 \mathrm{GeV}$, and $100-150 \mathrm{GeV}$ for each $K_{S}$ embedded $p_{T}$. This would result in three plots for each $K_{S} \quad p_{T}$ value: $K_{S}$ in $20-50 \mathrm{GeV}$ jets vs. $\phi_{j e t}, K_{S}$ in $50-100 \mathrm{GeV}$ jets vs. $\phi_{j e t}, K_{S}$ in $100-150$ $\mathrm{GeV}$ jets vs. $\phi_{\text {jet }}$. Unfortunately, statistics do not allow this since there are far too few embedded $K_{S}$ at each $p_{T}$ range to be subdivided into $5 \phi_{j e t}$ ranges and 3 jet $E_{T}$ ranges. Hence, groups of $K_{S}$ embedded $p_{T}$ are combined into three $K_{S}$ embedded $p_{T}$ groups given by the Table 7.2. The low $K_{S}$ embedded $p_{T}$ combines more embedded $p_{T}$ values than either the high or mid $K_{S}$ embedded $p_{T}$ groups because of the lower $K_{S}$ efficiency at the low $p_{T}$.

By combining embedded $p_{T}$ values, the $K_{S}$ in jet efficiency can be studied for $20-50 \mathrm{GeV}, 50-100 \mathrm{GeV}, 100-150 \mathrm{GeV}$ as a function of $\phi_{j e t}$ for three separate groups of $K_{S}$ embedded $p_{T}$ (low,mid,high). In Figure 7.12, there are three resulting plots for $\phi_{j e t}$ for the three groups. Although the $\phi_{j e t}$ efficiency is approximately constant for a given group of embedded $p_{T}$ ranges for $20-50 \mathrm{GeV}, 50-100 \mathrm{Gev}$, and 100-150 $\mathrm{GeV}$ jets (any plot in figure 7.12), the value of this constant varies for each group (low,mid,high) because the efficiency of $K_{S} \quad p_{T}$ varies for each group (i.e. low $p_{T}$ group has a lower efficiency than the mid $p_{T}$ group). 
Table 7.1: The groups of embedded $K_{S}$ in jets ranges

\begin{tabular}{|c|c|}
\hline $\begin{array}{c}\text { Groups of } \\
\text { Embedded } \\
K_{S} p_{T} \text { values }\end{array}$ & Embedded $p_{T}$ Values \\
\hline Low & $900 \mathrm{MeV}, 1.2 \mathrm{GeV}, 1.4 \mathrm{GeV}, 1.6 \mathrm{GeV}$ \\
& $1.8 \mathrm{GeV}, 2.0 \mathrm{GeV}, 2.3 \mathrm{GeV}, 2.6 \mathrm{GeV}$ \\
Mid & $2.9 \mathrm{GeV}, 3.0 \mathrm{GeV}, 3.5 \mathrm{GeV}, 4.0 \mathrm{GeV}, 5.0 \mathrm{GeV}$ \\
High & $6.0 \mathrm{GeV}, 7.0 \mathrm{GeV}, 8.0 \mathrm{GeV}, 9.0 \mathrm{GeV}, 10.0 \mathrm{GeV}$ \\
\hline
\end{tabular}

Table 7.2: The $p_{T}$ window ranges for a given $K_{S} p_{T}$

\begin{tabular}{|c|c|}
\hline$p_{T}$ Range of $K_{S}(\mathrm{GeV})$ & $p_{T}$ Window Range $(\mathrm{MeV})$ \\
\hline$p_{T} \leq 1.4$ & 25.0 \\
$1.4<p_{T} \leq 1.8$ & 30.0 \\
$1.8<p_{T} \leq 2.3$ & 50.0 \\
$2.3<p_{T} \leq 2.6$ & 75.0 \\
$2.6<p_{T} \leq 3.0$ & 100.0 \\
$3.0<p_{T} \leq 3.5$ & 150.0 \\
$3.5<p_{T} \leq 5.0$ & 200.0 \\
$5.0<p_{T} \leq 7.0$ & 500.0 \\
$7.0<p_{T} \leq 8.0$ & 750.0 \\
$8.0<p_{T} \leq 10.0$ & 1000.0 \\
\hline
\end{tabular}

For figure 7.10 , the $K_{S}$ efficiency as a decreasing function of jet energy is shown for each of the three groups. As a result, three separate $K_{S}$ efficiency curves will be made for $K_{S}$ in $20-50 \mathrm{GeV}, 50-100 \mathrm{GeV}$, and $100-150 \mathrm{GeV}$ jets. The $K_{S}$ efficiency as function of $\eta_{j e t}$ (Figure 1.11), luminosity (Figure 7.13), $\left|z_{\text {primary }}-z_{\text {jet }}\right|$ (Figure 7.14), and tracks per jet (Figure 7.15) are also shown. The $K_{S}$ efficiency curve for each jet $E_{T}$ integrates over all other efficiency dependencies. 
Table 7.3:

\begin{tabular}{|c|c|c|c|}
\hline Cuts & $\begin{array}{c}\text { Number of } K_{S} \\
\text { in MC }\end{array}$ & $\%$ & $\begin{array}{c}\% \text { in Data } \\
(1.5-1.7 \mathrm{GeV})\end{array}$ \\
\hline $\begin{array}{c}\text { After Event Cuts } \\
\chi^{2}<20.0\end{array}$ & $\begin{array}{l}495 . \pm 23 . \\
480+23\end{array}$ & $\begin{array}{c}1.032 \pm 0.0685 \\
1000\end{array}$ & $1.068 \pm 0.0946$ \\
\hline$\left|z_{K S^{-}} z_{\text {primary }}\right|<3.0 \mathrm{~cm}$ & 476. \pm 23 & $0.993 \pm 0.0663$ & $0.983 \pm 0.0857$ \\
\hline$p_{T}^{\text {tracks }}$ from $K S>500 \mathrm{MeV}$ & $288 . \pm 18$ & $0.601 \pm 0.0462$ & $0.692 \pm 0.0624$ \\
\hline$\left|\eta^{K S}\right|<1.0$ & $282 . \pm 17$ & $0.588 \pm 0.0454$ & $0.657 \pm 0.0593$ \\
\hline $3 \mathrm{D}$ Displacement ${ }^{K S}>1.0 \mathrm{~cm}$ & $258 . \pm 17$ & $0.537 \pm 0.0427$ & $0.629 \pm 0.0513$ \\
\hline $\cos \theta_{P D}^{K S}>0.990$ & $255 . \pm 16$ & $0.531 \pm 0.0423$ & $0.561 \pm 0.0428$ \\
\hline
\end{tabular}

Table 7.4: The number of $K_{S}$ after each successive cut for $p_{T}=1.6 \mathrm{GeV}$ inside $50-100 \mathrm{GeV}$ jets. The errors are taken from the fit of the area of the Gaussian.

\begin{tabular}{|c|c|c|c|}
\hline Cuts & $\begin{array}{l}\text { Number of } K_{S} \\
\text { in MC }\end{array}$ & $\%$ & $\begin{array}{c}\% \text { in Data } \\
(1.5-1.7 \mathrm{GeV})\end{array}$ \\
\hline After Event Cuts & $801 . \pm 30$ & $1.031 \pm 0.0550$ & $1.017 \pm 0.0816$ \\
\hline$\chi_{K S}^{2}<20.0$ & $777 . \pm 29$ & 1.000 & 1.000 \\
\hline$\left|z_{K S}-z_{\text {primary }}\right|<3.0 \mathrm{~cm}$ & $773 . \pm 29$ & $0.995 \pm 0.0534$ & $0.973 \pm 0.0753$ \\
\hline$p_{T}^{\text {tracks from } K S}>500 \mathrm{MeV}$ & $522 . \pm 24$ & $0.672 \pm 0.0398$ & $0.644 \pm 0.0536$ \\
\hline$\left|\eta^{K S}\right|<1.0$ & $508 . \pm 23$ & $0.654 \pm 0.0390$ & $0.598 \pm 0.0506$ \\
\hline $3 \mathrm{D}$ Displacement ${ }^{K S}>1.0 \mathrm{~cm}$ & $462 . \pm 22$ & $0.595 \pm 0.0364$ & $0.571 \pm 0.0427$ \\
\hline $\cos \theta_{P D}^{K S}>0.990$ & 459. \pm 22 . & $0.592 \pm 0.0362$ & $0.512 \pm 0.0369$ \\
\hline
\end{tabular}


Table 7.5: The number of $K_{S}$ after each successive cut for $p_{T}=1.6 \mathrm{GeV}$ inside $100-150 \mathrm{GeV}$ jets. The errors are taken from the fit of the area of the Gaussian.

\begin{tabular}{|c|c|c|c|}
\hline Cuts & $\begin{array}{c}\text { Number of } K_{S} \\
\text { in MC }\end{array}$ & $\%$ & $\begin{array}{c}\text { \% in Data } \\
(1.5-1.7 \mathrm{GeV})\end{array}$ \\
\hline $\bar{A}$ & $578 . \pm 25$ & $9 \pm 0$ & $1.069 \pm 0.0975$ \\
\hline$x^{2}$ & 25. & $1 .($ & 1.000 \\
\hline$z_{p}$ & $564 . \pm 25$. & $0.994 \pm$ & $0.971 \pm 0.0848$ \\
\hline$>500 \mathrm{MeV}$ & 373 & $.656 \pm$ & $0.656 \pm 0.0604$ \\
\hline & 20. & 14 & $0.634 \pm 0.0588$ \\
\hline isplacemen & $343 .=$ & $0.603 \pm 0$. & $0.563 \pm 0.0477$ \\
\hline $\cos \theta_{P D}^{K S}>0.990$ & $339 . \pm 18$. & $0.597 \pm 0.0420$ & $0.510 \pm 0.0391$ \\
\hline
\end{tabular}

Table 7.6: The number of $K_{S}$ after each successive cut for $p_{T}=6.0 \mathrm{GeV}$ in $20-50$ $\mathrm{GeV}$ jets. The errors are taken from the fit of the area of the Gaussian.

\begin{tabular}{|c|c|c|c|}
\hline Cuts & $\begin{array}{c}\text { Number of } K_{S} \\
\text { in } \mathrm{MC}\end{array}$ & $\%$ & $\begin{array}{c}\text { \% in Data } \\
(5.5-6.5 \mathrm{GeV})\end{array}$ \\
\hline After Ever & $515 . \pm 27$ & $1.010 \pm 0.0744$ & $1.006 \pm 0.0726$ \\
\hline$\gamma^{2}$ & 27. & 1.000 & 1.000 \\
\hline$\left|z_{K S^{-}} z_{\text {primary }}\right|<3.0 \mathrm{~cm}$ & $508 . \pm 26$ & $0.997 \pm 0.0732$ & $0.976 \pm 0.0701$ \\
\hline$p_{T}^{\text {tracks from } K \dot{S}}>500 \mathrm{MeV}$ & $508 . \pm 26$ & $0.997 \pm 0.0732$ & $1.039 \pm 0.0746$ \\
\hline$\left|\eta^{K S}\right|<1.0$ & $=26$. & $0.971 \pm$ & $0.996 \pm 0.0725$ \\
\hline $3 \mathrm{D} \mathrm{I}$ & 48 & $0.957 \pm 0$ & $0.968 \pm 0.0707$ \\
\hline & $487 . \pm 26$. & $0.955 \pm 0.0716$ & $0.956 \pm 0.0672$ \\
\hline
\end{tabular}


Table 7.7: The number of $K_{S}$ after each successive cut for $p_{T}=6.0 \mathrm{GeV}$ in $50-100$ $\mathrm{GeV}$ jets. The errors are taken from the fit of the area of the Gaussian.

\begin{tabular}{|c|c|c|c|}
\hline Cuts & $\begin{array}{c}\text { Number of } K_{S} \\
\text { in } M C\end{array}$ & $\%$ & $\begin{array}{c}\% \text { in Data } \\
(5.5-6.5 \mathrm{GeV})\end{array}$ \\
\hline After Event Cuts & $868 . \pm 35$ & $1.000 \pm 0.0569$ & $1.032 \pm 0.0869$ \\
\hline$\chi_{K S}^{2}<20.0$ & $868 . \pm 35$ & 1.000 & 1.000 \\
\hline$\left|z_{K S}-z_{\text {primary }}\right|<3.0 \mathrm{~cm}$ & 866. 土35. & $0.998 \pm 0.0564$ & $0.982 \pm 0.0800$ \\
\hline$p_{T}^{\text {tracks from } K S}>500 \mathrm{MeV}$ & 867. \pm 35 . & $0.999 \pm 0.0565$ & $1.123 \pm 0.0914$ \\
\hline$\left|\eta^{K S}\right|<1.0$ & 847. \pm 34 & $0.976 \pm 0.0554$ & $1.089 \pm 0.0875$ \\
\hline 3D Displacement ${ }^{K S}>1.0 \mathrm{~cm}$ & 823. \pm 33 & $0.948 \pm 0.0540$ & $1.046 \pm 0.0812$ \\
\hline $\cos \theta_{P D}^{K S}>0.990$ & $820 . \pm 33$ & $0.944 \pm 0.0535$ & $1.010 \pm 0.0778$ \\
\hline
\end{tabular}

Table 7.8: The number of $K_{S}$ after each successive cut for $p_{T}=6.0 \mathrm{GeV}$ in $100-150$ $\mathrm{GeV}$ jets. The errors are taken from the fit of the area of the Gaussian.

\begin{tabular}{|c|c|c|c|}
\hline Cuts & $\begin{array}{c}\text { Number of } K_{S} \\
\text { in } \mathrm{MC}\end{array}$ & $\%$ & $\begin{array}{c}\% \text { in Data } \\
(5.5-6.5 \mathrm{GeV})\end{array}$ \\
\hline After Event Cuts & $603 . \pm 29$. & $1.015 \pm 0.0698$ & $0.993 \pm 0.0886$ \\
\hline$\chi_{K S}^{2}<20.0$ & 094. 士 29. & 1.000 & 1.000 \\
\hline$\left|z_{K S}-z_{\text {primary }}\right|<3.0 \mathrm{~cm}$ & $592 . \pm 29$ & $0.997 \pm 0.0686$ & $0.969 \pm 0.0860$ \\
\hline$p_{T}^{\text {tracks from } K S}>500 \mathrm{MeV}$ & $597 . \pm 29$ & $1.004 \pm 0.0689$ & $1.144 \pm 0.1029$ \\
\hline$\left|\eta^{K S}\right|<1.0$ & $580 . \pm 28$ & $0.976 \pm 0.0674$ & $1.124 \pm 0.1016$ \\
\hline $3 \mathrm{D}$ Displacement ${ }^{K S}>1.0 \mathrm{~cm}$ & $570 . \pm 28$ & $0.960 \pm 0.0662$ & $1.062 \pm 0.0917$ \\
\hline $\cos \theta_{P D}^{K S}>0.990$ & $570 . \pm 28$ & $0.959 \pm 0.0660$ & $1.029 \pm 0.0881$ \\
\hline
\end{tabular}




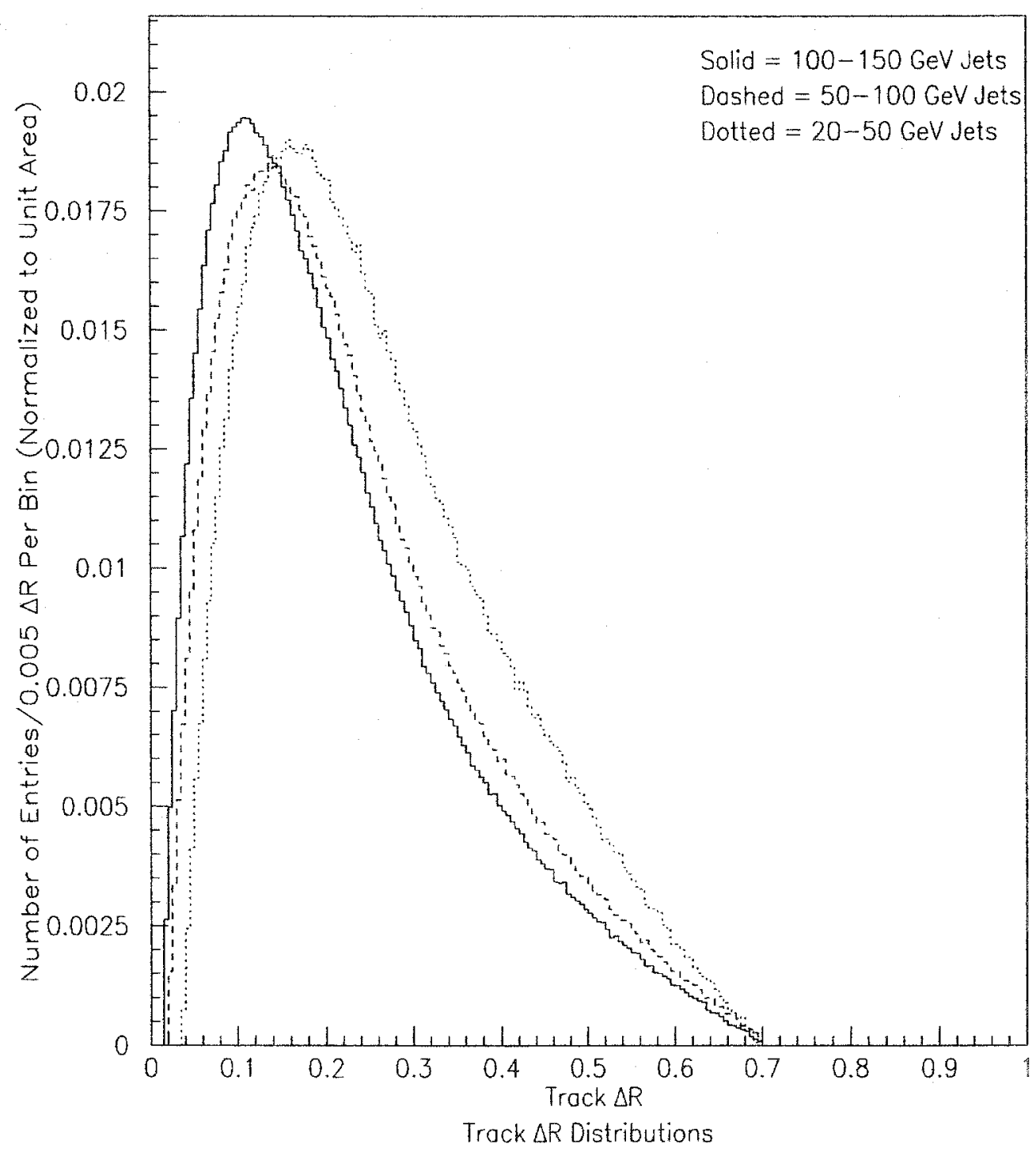

Figure 7.1: A few $\left|\Delta R_{\text {jet-track }}\right|$ distributions normalized to unit area. In order of decreasing peaks, the $\left|\Delta R_{\text {jet-track }}\right|$ distribution inside jet energies of $100-150 \mathrm{GeV}$, between $50 \mathrm{GeV}$ and $100 \mathrm{GeV}$, and between $20 \mathrm{GeV}$ and $50 \mathrm{GeV} . K_{S}$ are embedded into jets according to $\left|\Delta R_{\text {jet-track }}\right|$ distributions. 


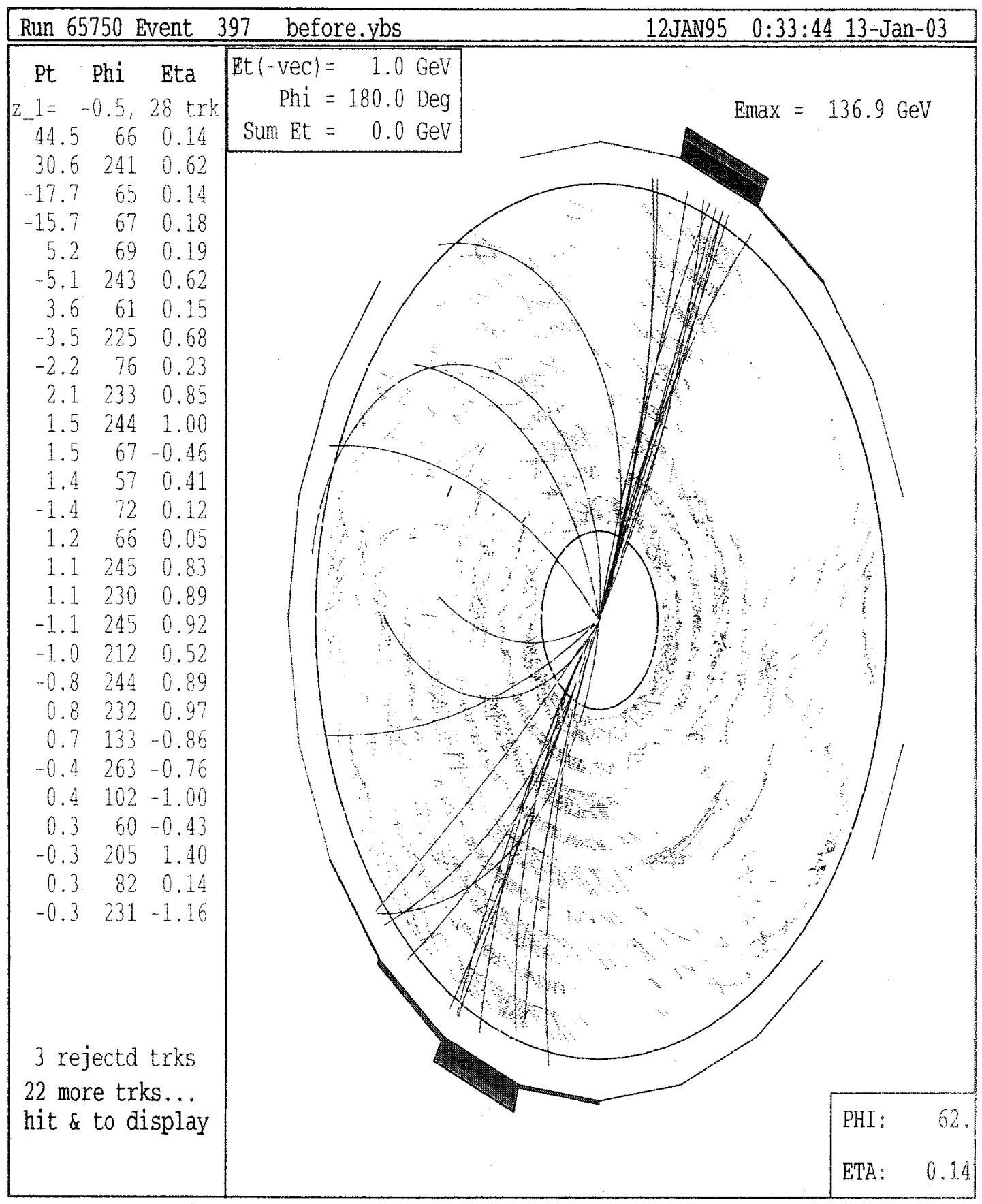

Figure 7.2: A transverse view of a jet event. A $K_{S}$ has not yet been track embedded. 


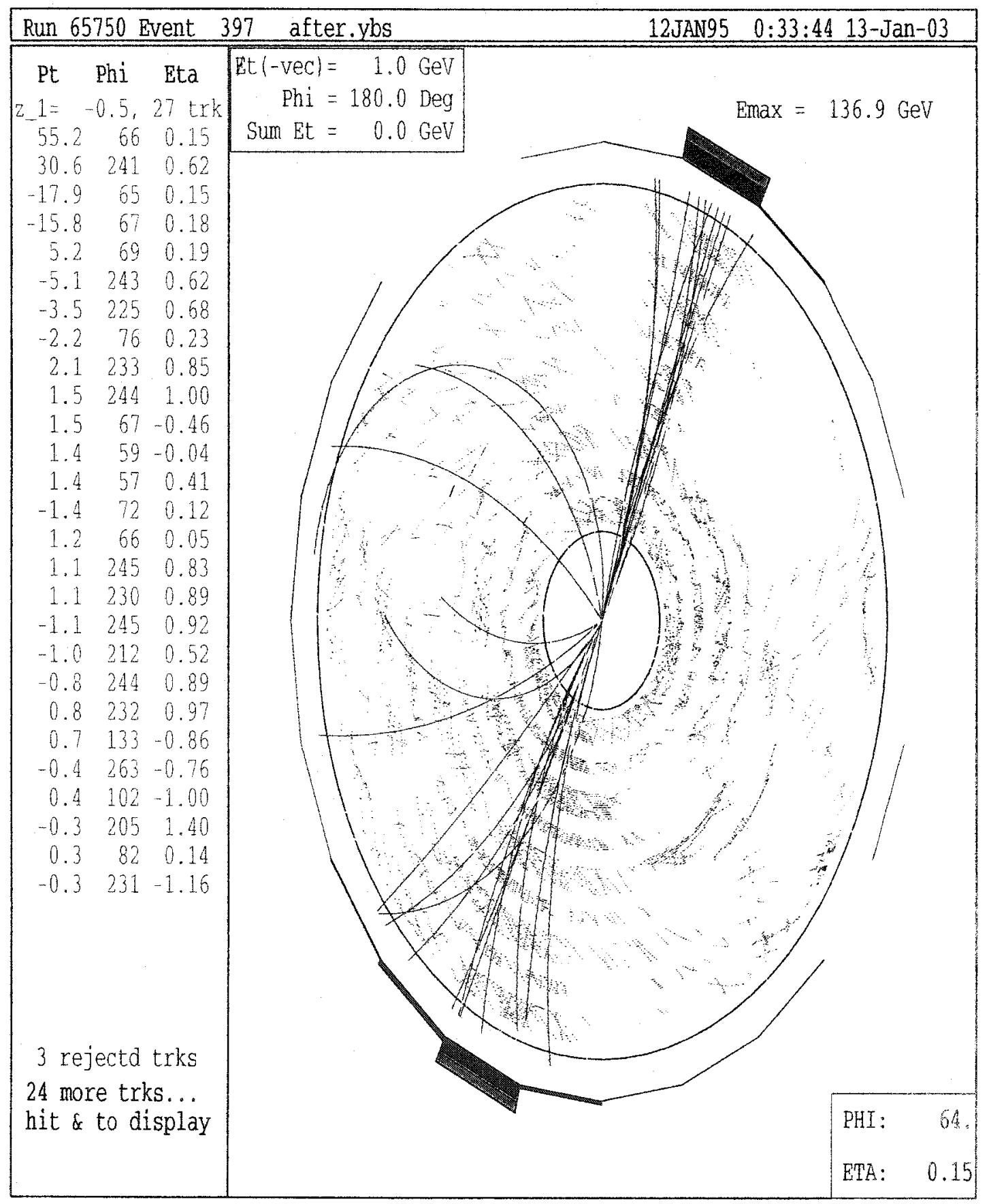

Figure 7.3: A transverse view of a jet event with a $3.0 \mathrm{GeV}$ track-embedded $K_{S}$. The $K_{S}$ consists of a straight track and a curved track not seen in Figure 7.1. The straight track is the higher $p_{T}$ pion track, and the curved track is the softer $p_{T}$ pion track. 


\subsection{Comparison with Jets in Data}

Tables 7.3-7.8 compare after each cut the embedded $K_{S}$ at a given $p_{T}$ value in jets to $K_{S}$ in jets within a particular $p_{T}$ range in the data. The $1.6 \mathrm{GeV}$ and 6.0 GeV embedded $K_{S}$ in the track-embedding are matched with $K_{S}$ having $p_{T}$ ranges within $1.5-1.7 \mathrm{GeV}$ and $5.5-6.5 \mathrm{GeV}$. This is done again for $20-50 \mathrm{GeV}, 50-100 \mathrm{GeV}$, and $100-150 \mathrm{GeV}$ jet $E_{T}$ ranges. For the $6.0 \mathrm{GeV}$ case, the agreement is quite good whereas for the $1.6 \mathrm{GeV}$ case, the data and MC agree to approximately $15 \%$. The disagreement either appears to originate with the track cut and continues throughout the rest of the table or appears at the end of the table with the appearance of the pointing cut. The comparisons are normalized with respect to $K_{S}$ in jets pointing to the primary vertex (i.e. the $\chi_{K S}^{2}<20.0 \mathrm{cut}$ ). This discrepancy is not fully understood although it has been studied.

\subsection{Single Track Efficiency}

The primary reason to why the single track in jets efficiencies are calculated is in order to correct the single track $p_{T}$ spectra so that $\frac{N_{\text {track }}}{\text { jet }}$ number may be computed (done in later Chapter 8). This number can also be compared with the $\frac{N_{K S}}{j e t}$. Another reason is a cross-check for the $K_{S}$ efficiency curves since the track efficiency in jets has been studied. In addition, the product of the single track efficiencies along with the secondary vertex reconstruction efficiency should yield the $K_{S}$ efficiency.

The procedure of single track-embedding is very similar to the procedure of the $K_{S}$ in jet track-embedding with the exception of instead of embedding 2 pion tracks from the $K_{S}$ at a particular $p_{T}$ with the $K_{S}$ directed according to the $\Delta R_{\text {jet-track }}$ distribution, a single pion track at a particular $p_{T}$ is embedded into a jet according 
to the $\Delta R_{\text {jet-track }}$ distribution. Moreover, the text files of pions at a particular $p_{T}$ are produced rather than $K_{S}$ at a particular $p_{T}$. To compute each efficiency point, background subtraction is applied to the $p_{T}$ to yield the number of pions found at that particular $p_{T}$. Typically, the single track efficiency is done separately for positive and negative pions, but since the efficiencies converge above $400 \mathrm{MeV}$, the single track efficiency uses only negative pions.

In Figure 7.19, the single track efficiency vs. $p_{T}$ for tracks inside $20-50 \mathrm{GeV}, 50-$ $100 \mathrm{GeV}$, and $100-150 \mathrm{GeV}$ is shown. The single track is approximately a constant of $95 \%$ for $20-50 \mathrm{GeV}$ and $100-150 \mathrm{GeV}$ ranges whereas the single track efficiency is about $90 \%$ for the $50-100 \mathrm{GeV}$ range.

It should be noted that aside from the track-embedding references in Chapter $5,[28][42][51]$ [54] [55] [56] [57] [58], the only analysis which embeds tracks in jets is [28]. This method applied here closely resembles this analysis with the exception that tracks (and $K_{S}$ ) are embedded according to the track density distributions and jet vertices. However, the single track efficiencies, even with different cuts, are similar as well as their dependence on $\eta$ and $\phi$ variables. The comparisons are good to $5-10 \%$. 



Figure 7.4: The mass distribution of $K_{S}$ candidates after track-embedding. The mass distributions of $1.6 \mathrm{GeV}$ (top left) and $6.0 \mathrm{GeV}$ (top right) $K_{S}$ candidates that pass (dashed line) and fail (solid line) $p_{T}$ window. The $p_{T}$ windows are 30 $\mathrm{MeV}$ and $0.5 \mathrm{GeV}$ for $1.6 \mathrm{GeV}$ and $6.0 \mathrm{GeV} K_{S}$. Below are the fitted background subtracted mass distributions. 


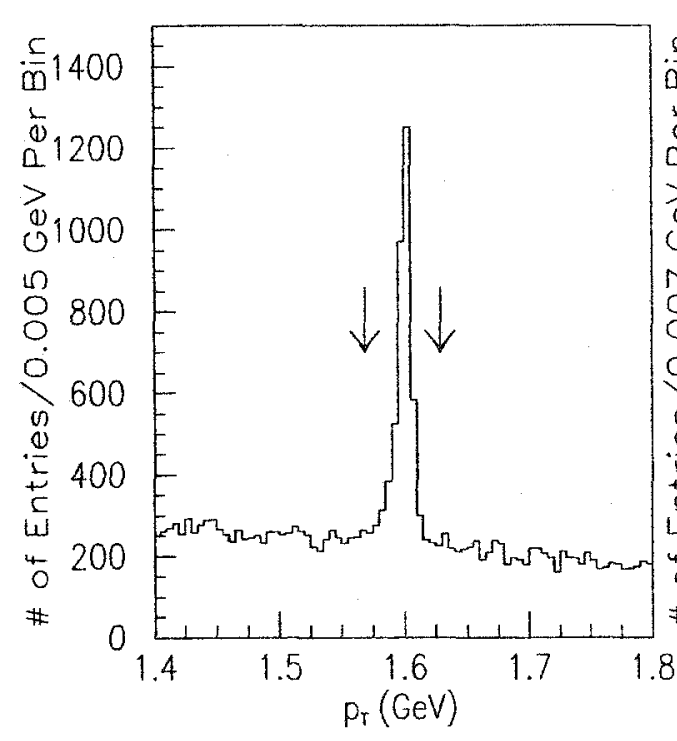

$1.6 \mathrm{GeV} \mathrm{K}_{\mathrm{s}}$ Embedded


6.0 GeV K $\mathrm{K}_{\mathrm{s}}$ Embedded

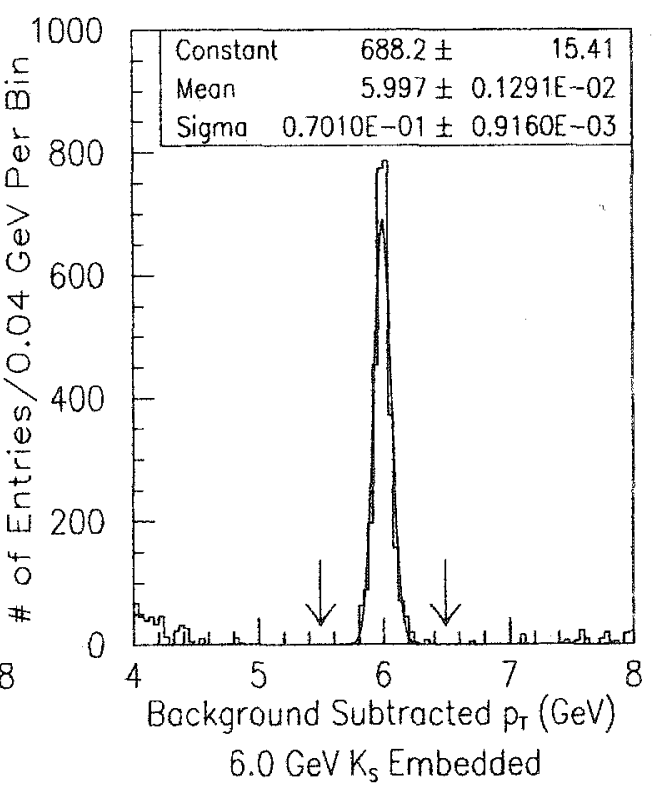

Figure 7.5: The $p_{T}$ distributions of $K_{S}$ candidates after track-embedding. The $p_{T}$ distributions of $1.6 \mathrm{GeV}$ (top left) and $6.0 \mathrm{GeV}$ (top right) $K_{S}$ candidates that pass (dashed line) and fail (solid line) $p_{T}$ window. The $p_{T}$ windows are $30 \mathrm{MeV}$ and $0.5 \mathrm{GeV}$ for $1.6 \mathrm{GeV}$ and $6.0 \mathrm{GeV} K_{S}$. Below are the fitted background subtracted $p_{T}$ distributions. 



Figure 7.6: In the top half, the $z_{\text {primary }}-z_{\text {jet }}$ distributions of the $1.6 \mathrm{GeV}$ (left) and $6.0 \mathrm{GeV}$ (right) candidates which pass (dashed line) and fail (solid line) the $p_{T}$ window cut. The arrows indicate the $\left|z_{\text {primary }}-z_{\text {jet }}\right|<5.0 \mathrm{~cm}$ cut. In the bottom half, the $z_{\text {jet }}^{r m s}$ distributions are shown similarly with the arrows indicating the $z_{\text {jet }}^{r m s}<5.0$ cut. 

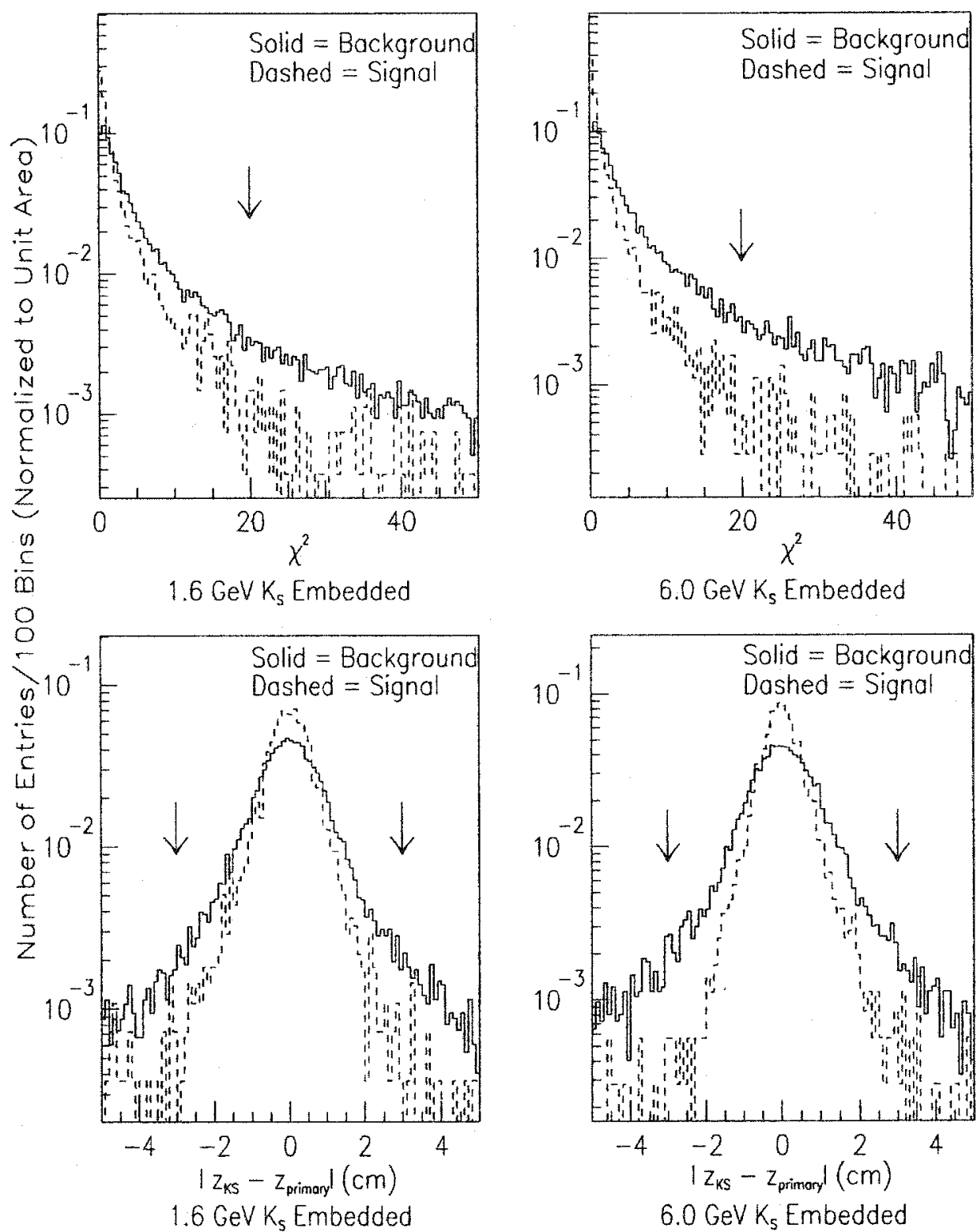

Figure 7.7: In the top half, the $\chi_{K S}^{2}$ distributions of the $1.6 \mathrm{GeV}$ (left) and 6.0 $\mathrm{GeV}$ (right) candidates which pass (dashed line) and fail (solid line) the $p_{T}$ window cut. The arrows indicate the $\chi_{K S}^{2}<20.0$ cut. In the bottom half, the $\left|z_{K S}-z_{\text {primary }}\right|$ distributions are shown similarly with the arrows indicating the $\left|z_{K S^{-}} z_{\text {primary }}\right|<3.0$ cm cut. 

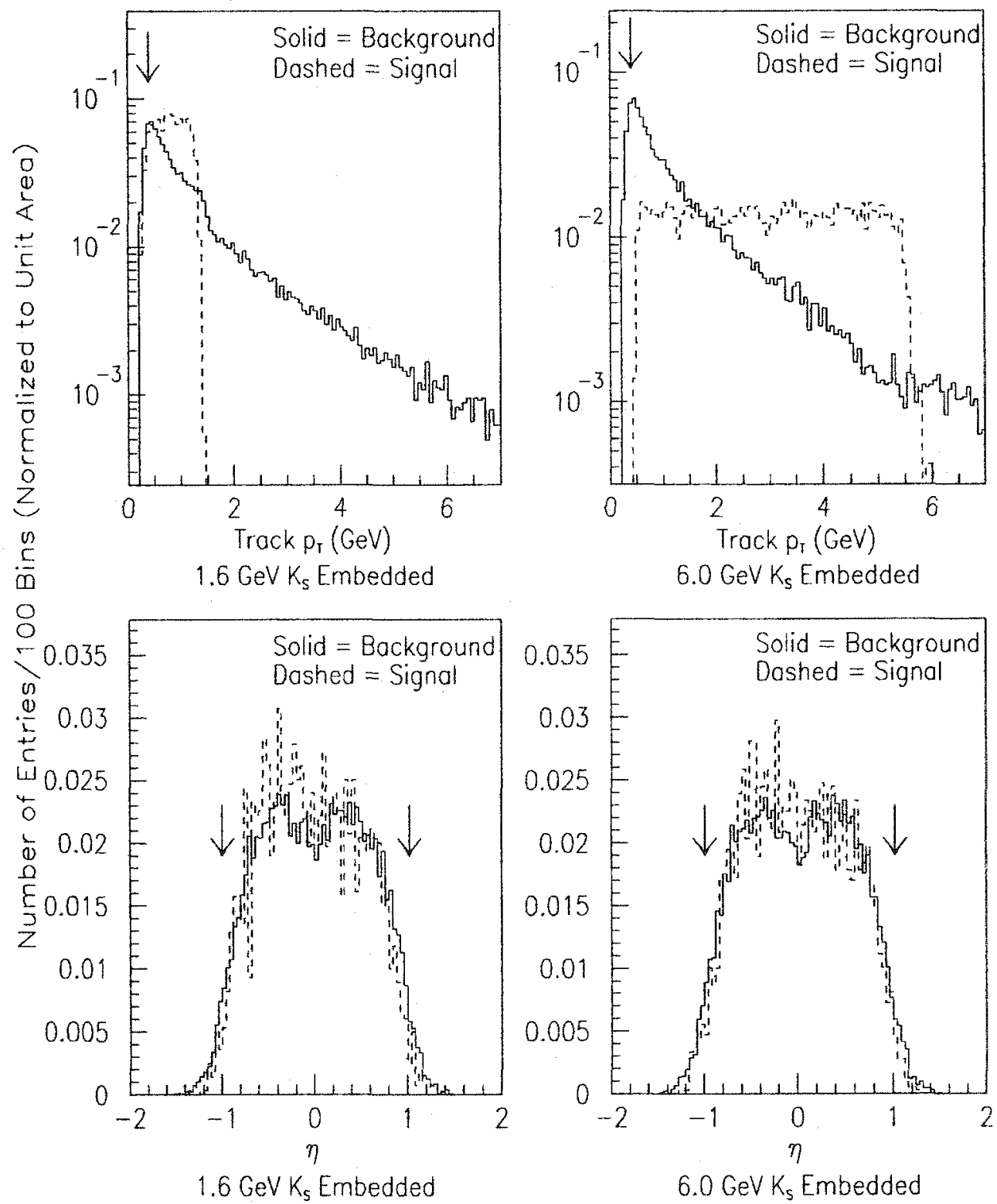

Figure 7.8: In the top half, the daughter track $p_{T}$ distributions of the $1.6 \mathrm{GeV}$ (left) and $6.0 \mathrm{GeV}$ (right) candidates which pass (dashed line) and fail (solid line) the $p_{T}$ window cut. The arrows indicate the $p_{T}^{\text {tracks }}$ from $K S>500.0 \mathrm{MeV}$ cut. In the bottom half, the $\eta^{K S}$ distributions are shown similarly with the arrows indicating the $\left|\eta^{K S}\right|<1.0$ cut. 

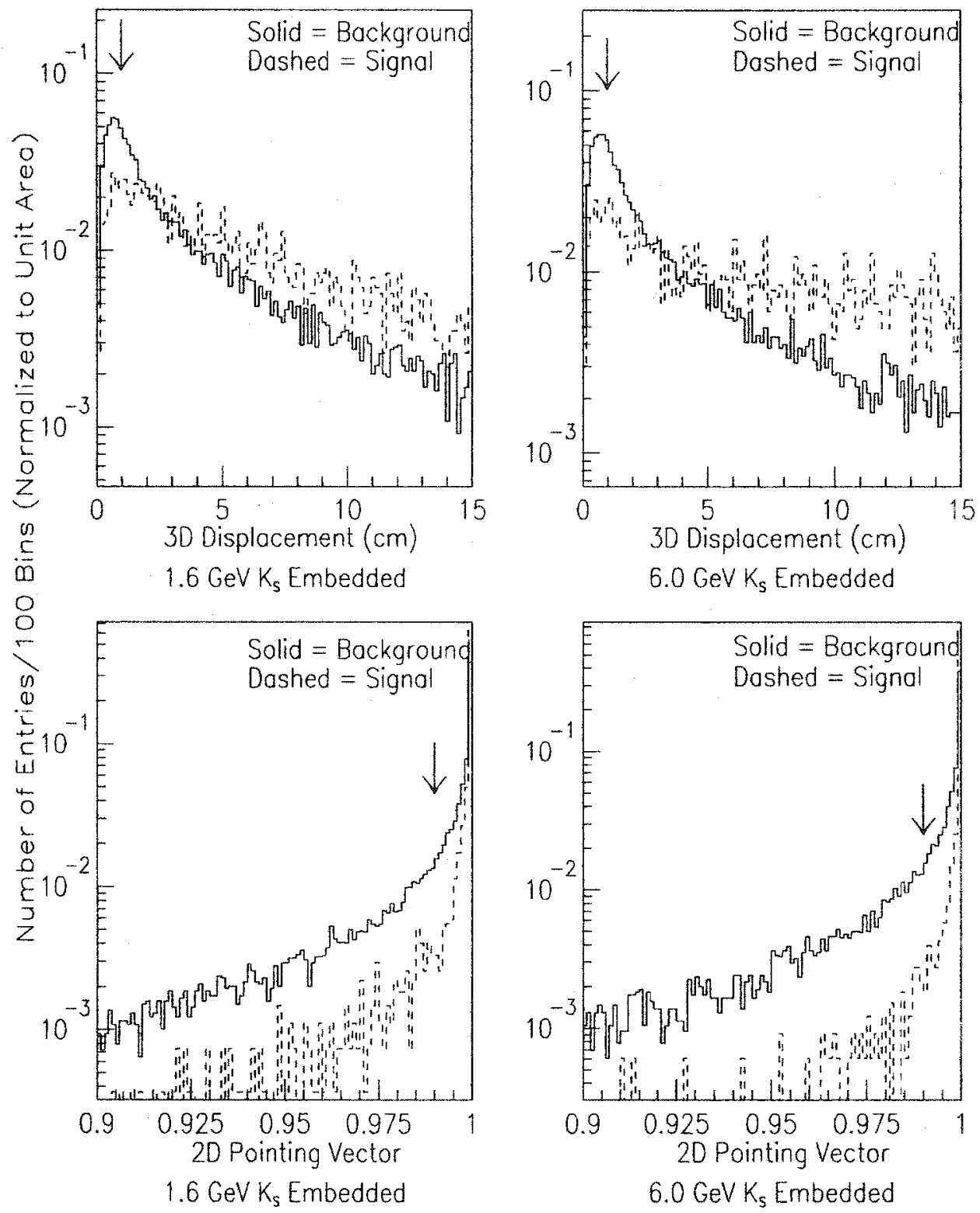

Figure 7.9: In the top half, the 3D displacement ${ }^{K S}$ distributions of the $1.6 \mathrm{GeV}$ (left) and 6.0 GeV (right) candidates which pass (dashed line) and fail (solid line) the $p_{T}$ window cut. The arrows indicate the $3 \mathrm{D}$ displacement ${ }^{K S}>1.0 \mathrm{~cm}$ cut. In the bottom half, the $2 \mathrm{D}$ pointing vector $\left(\cos \theta_{P D}^{K S}\right)$ distributions are shown similarly with the arrows indicating the $\cos \theta_{P D}^{K S}>0.990$ cut. 


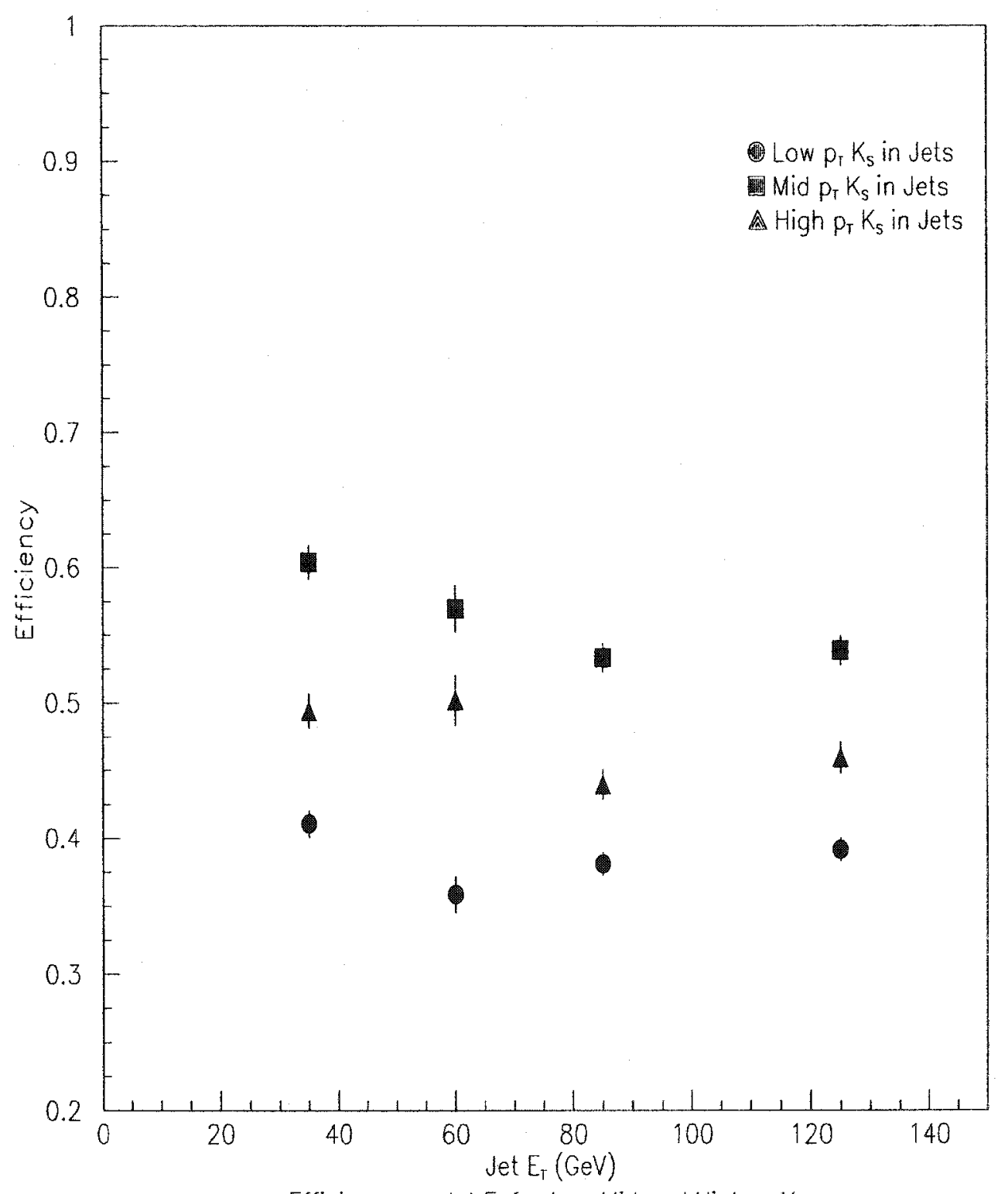

Efficiency vs. Jet $E_{T}$ for Low, Mid, and High $p_{T} K_{S}$

Figure 7.10: The $K_{S}$ efficiency vs. jet $E_{T}$ curve for the 3 groups of $K_{S} \quad p_{T}$ (low,mid,and high). The offsets in efficiency of each group to each other is due to the $p_{T}$ efficiency of the $K_{S}$ whereas the decrease in efficiency of every group is due to a jet $E_{T}$ efficiency dependence that will taken into account with 3 separate $K_{S}$ efficiency curves. 

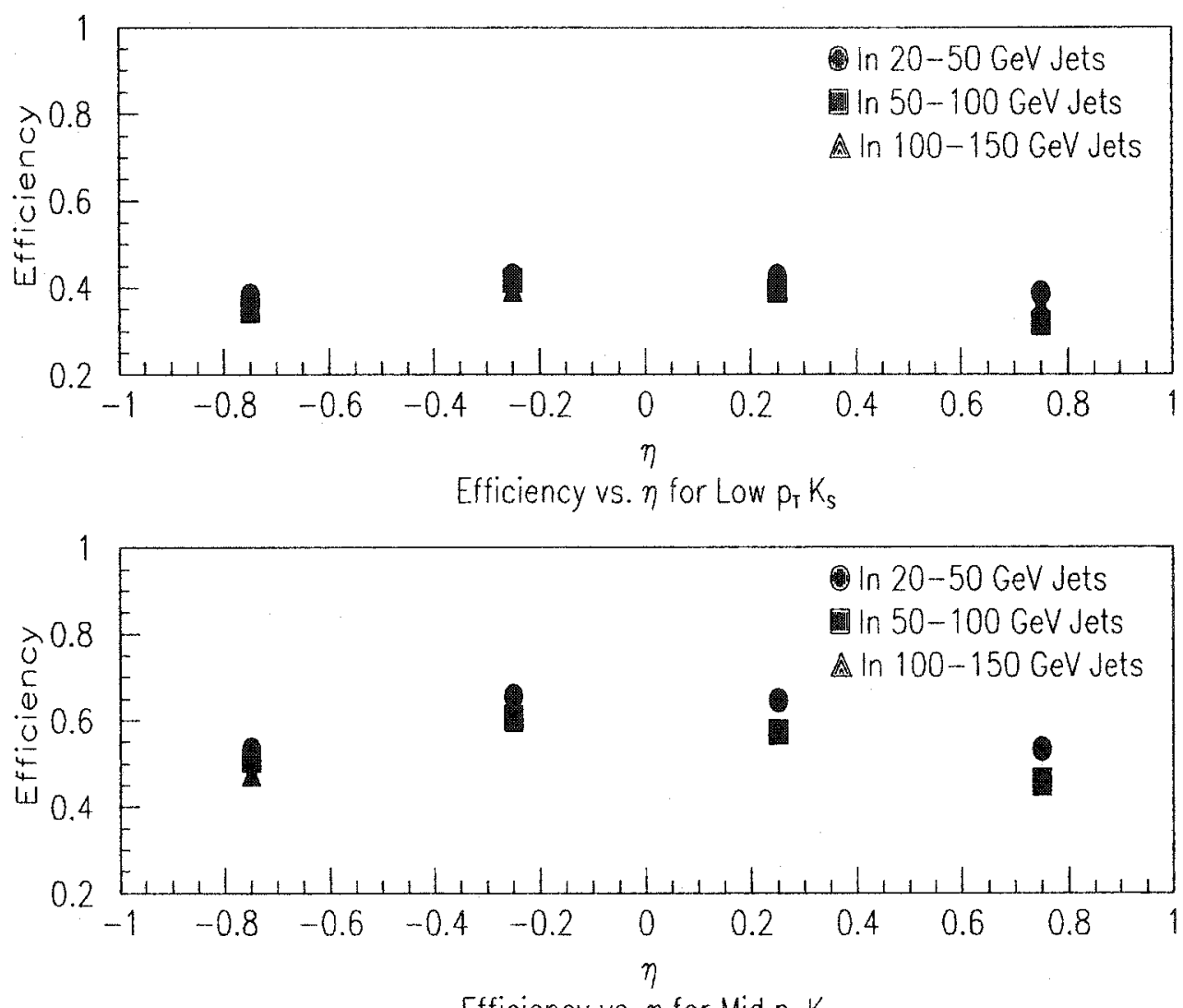

Efficiency vs. $\eta$ for Mid $p_{T} K_{S}$

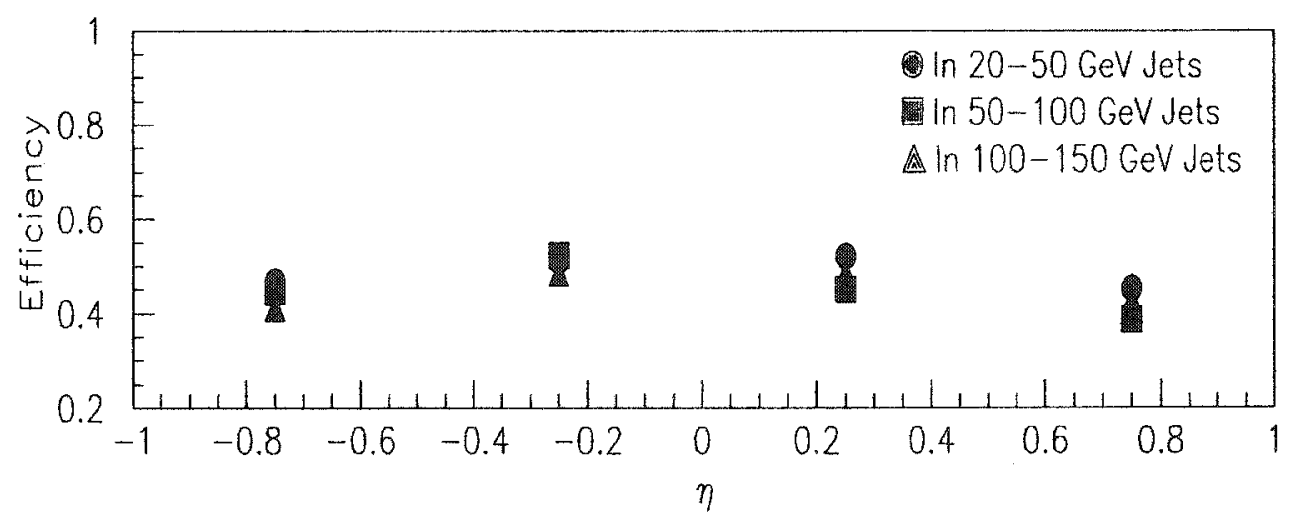

Efficiency vs. $\eta$ for High $p_{\mathrm{T}} \mathrm{K}_{\mathrm{s}}$

Figure 7.11: The $K_{S}$ efficiency vs. $\eta_{j e t}$ curve for the 3 groups of $K_{S} p_{T}$ (low, mid, and high). 


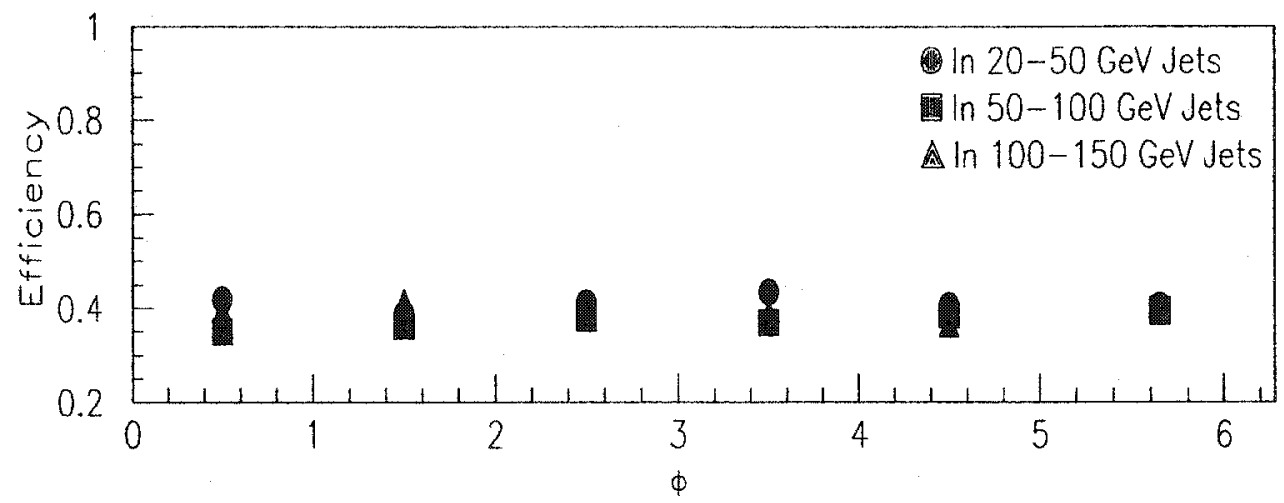

Efficiency vs. $\phi$ for Low $p_{\mathrm{T}} K_{\mathrm{S}}$



Efficiency vs. $\phi$ for Mid $p_{T} K_{S}$

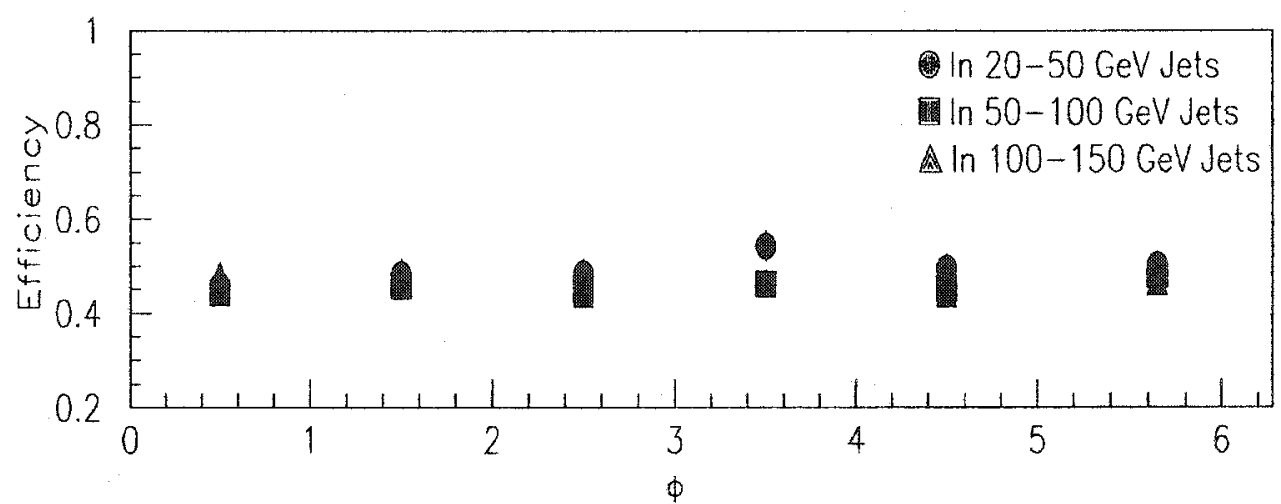

Efficiency vs. $\phi$ for High $p_{\mathrm{T}} K_{\mathrm{s}}$

Figure 7.12: The $K_{S}$ efficiency vs. $\phi_{j e t}$ curve for the 3 groups of $K_{S} p_{T}$ (low,mid,and high). 


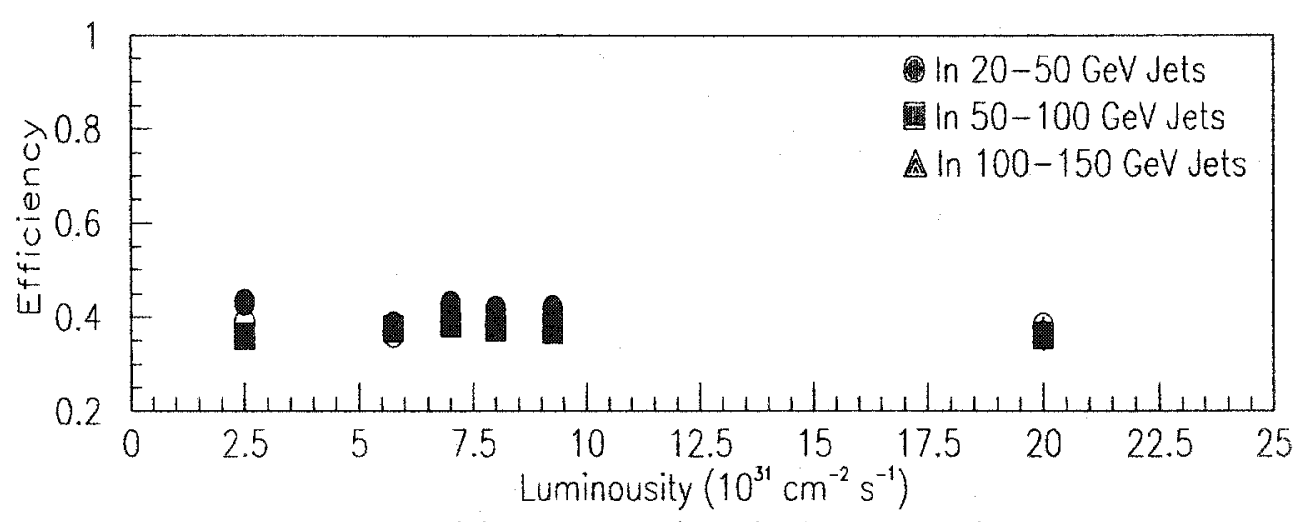

Efficiency vs. Luminousity for Low $p_{T}$ KS

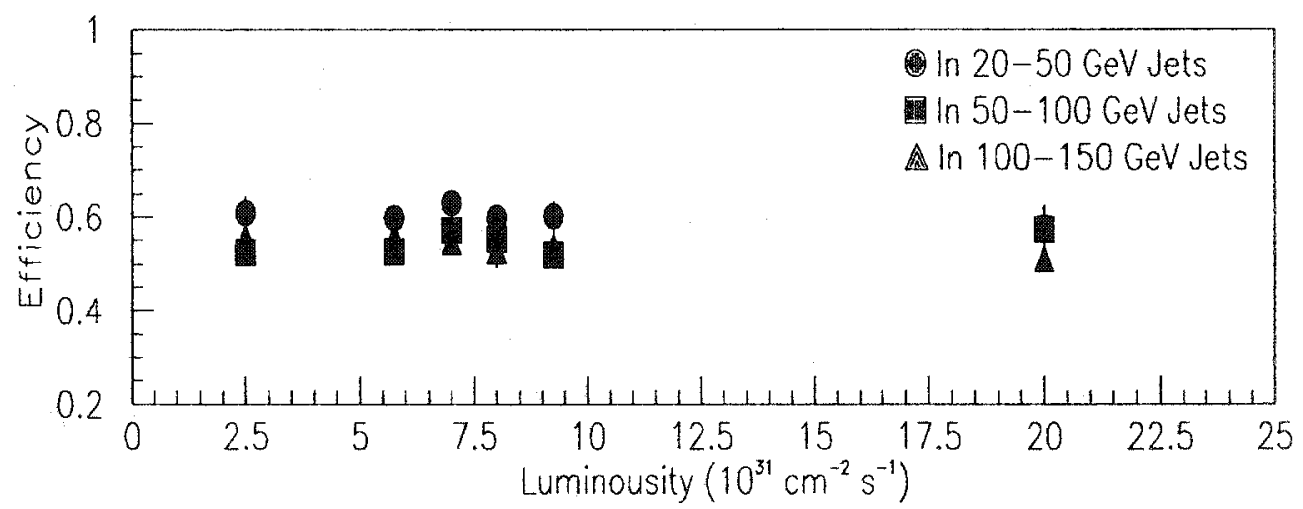

Efficiency vs. Luminousity for Mid $p_{\mathrm{r}} \mathrm{KS}$

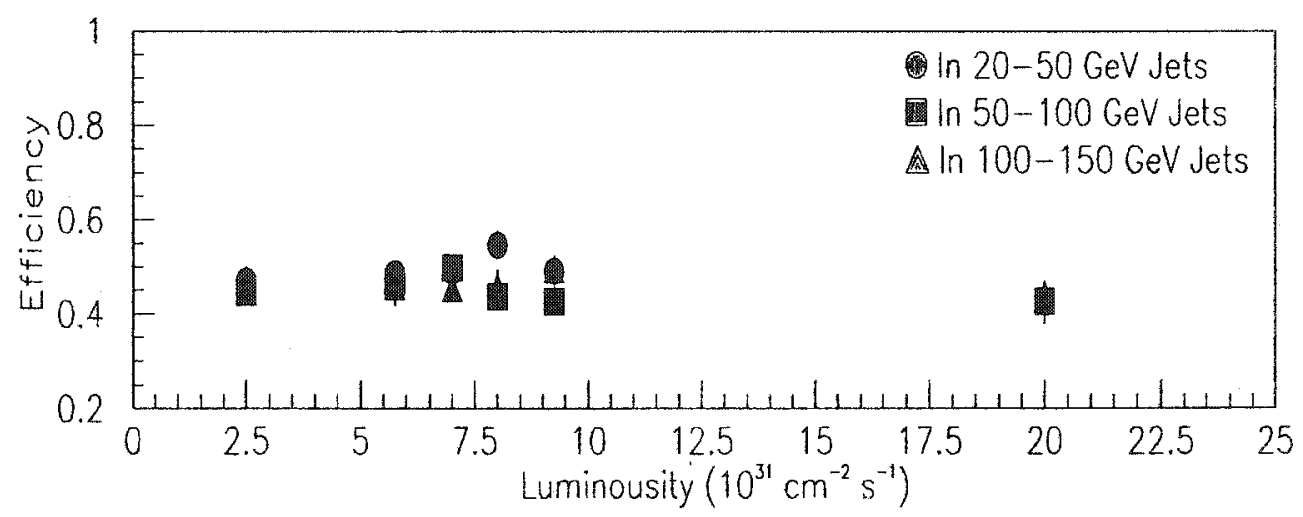

Efficiency vs. Luminousity for High $p_{T} K S$

Figure 7.13: The $K_{S}$ efficiency vs. luminosity curve for the 3 groups of $K_{S} p_{T}$ (low,mid, and high). 




Efficiency vs. $\left|z_{\text {primary }}-z_{\text {jel }}\right|$ for Low $p_{T} K_{S}$

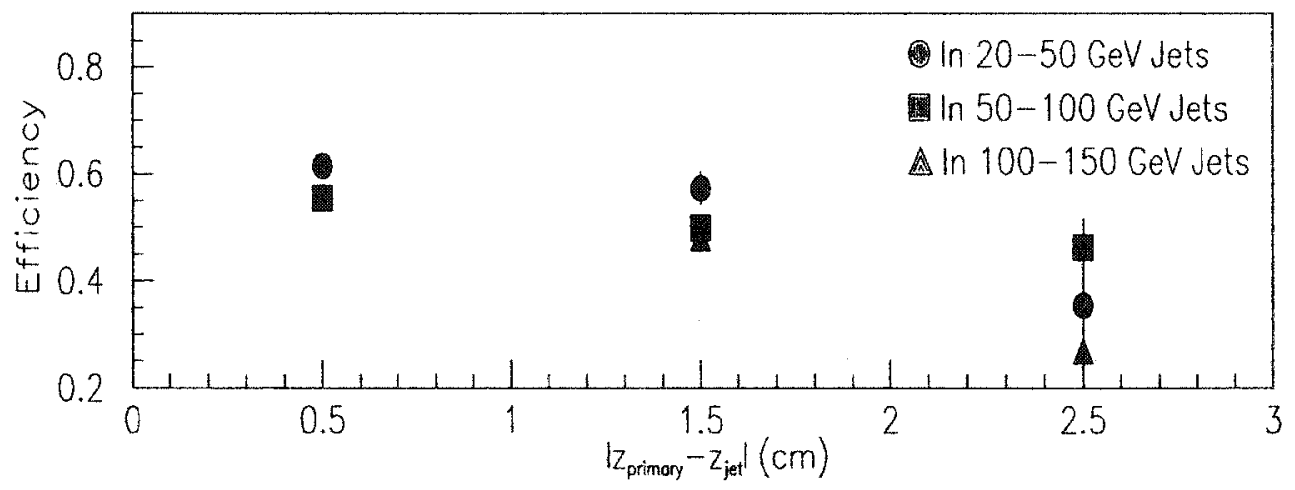

Efficiency vs. $\left|z_{\text {primery }}-z_{\text {jet }}\right|$ for Mid $p_{T} K_{S}$



Efficiency vs. $\left|z_{\text {primary }}-z_{\text {jet }}\right|$ for High $p_{T} K_{S}$

Figure 7.14: The $K_{S}$ efficiency vs. $\left|z_{\text {primary }}-z_{\text {jet }}\right|$ curve for the 3 groups of $K_{S} p_{T}$ (low, mid, and high). 


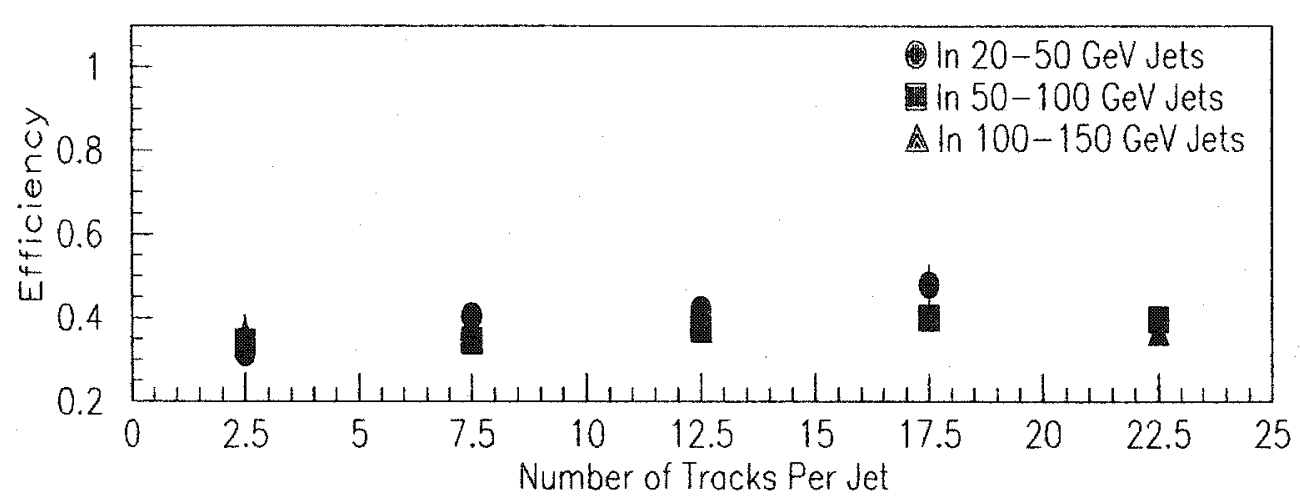

Efficiency vs. Number of Tracks Per Jet for Low $p_{r} K_{s}$



Efficiency vs. Number of Tracks Per Jet for Mid $p_{\mathrm{T}} K_{\mathrm{S}}$



Efficiency vs. Number of Trocks Per Jet for High $p_{1} K_{s}$

Figure 7.15: The $K_{S}$ efficiency vs. the number of tracks per jet curve for the 3 groups of $K_{S} \quad p_{T}$ (low,mid, and high). 


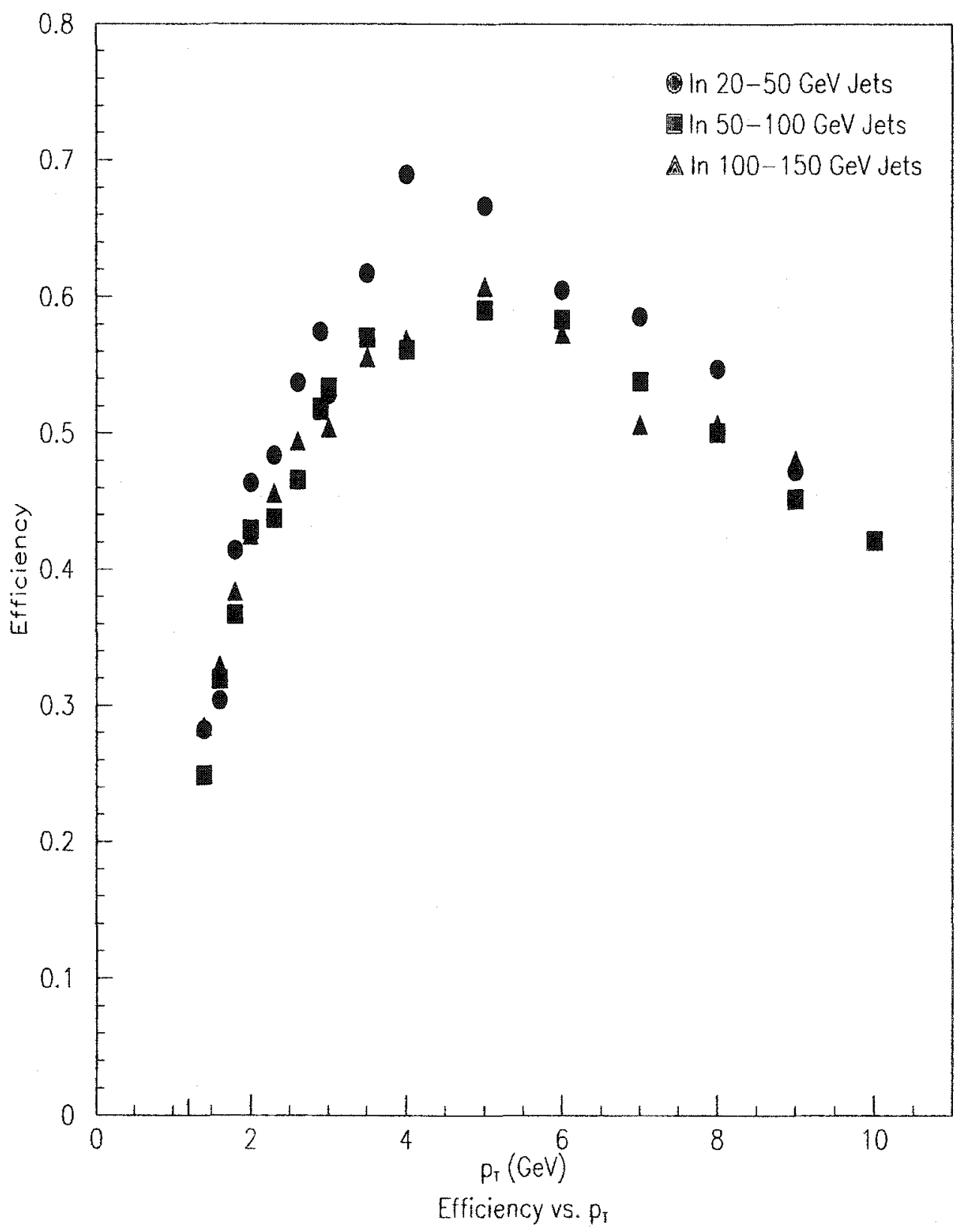

Figure 7.16: The $K_{S}$ efficiency vs. $p_{T}$ curve for $K_{S}$ inside jets. Each point in the top figure represents a $p_{T}$ value which implemented track-embedding. All three jet $E_{T}$ ranges are shown. 


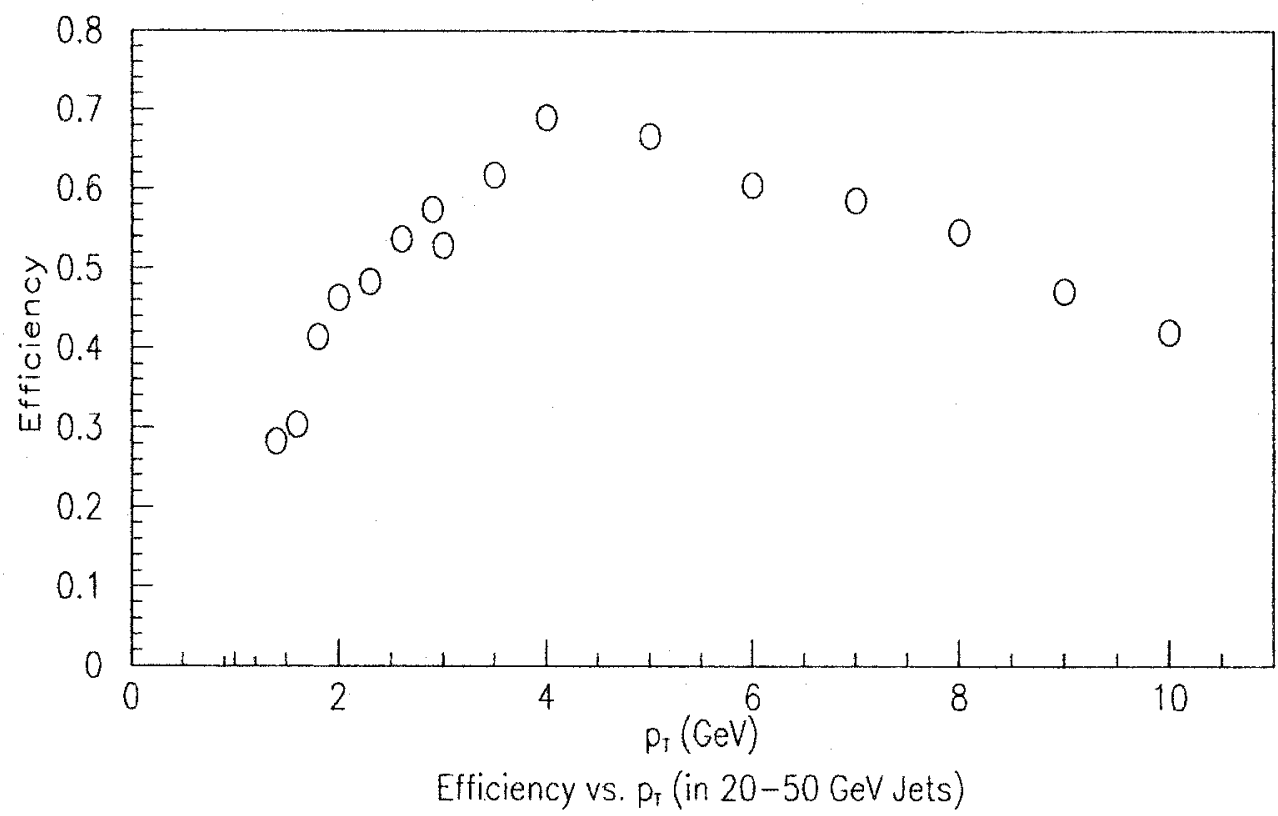

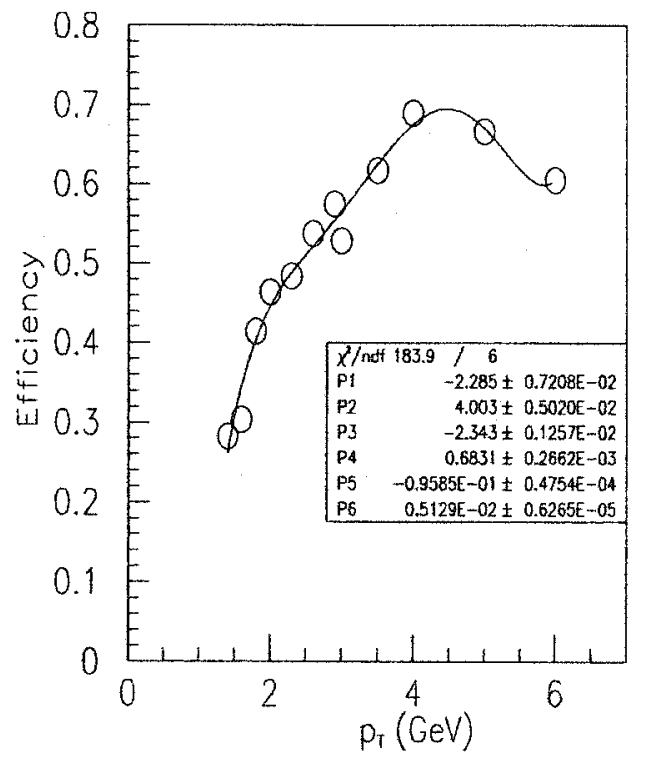

Efficiency vs. $p_{r}$ (in $20-50 \mathrm{GeV}$ Jets)

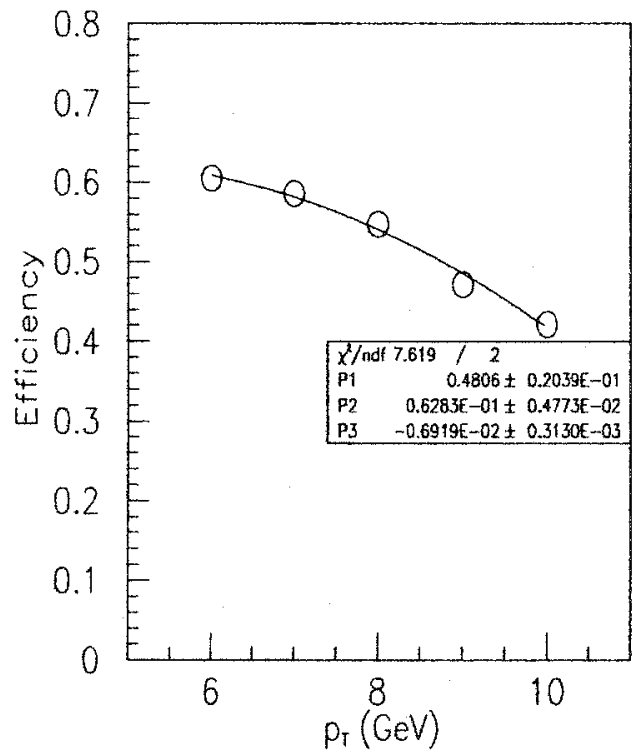

Efficiency vs. $p_{T}$ (in 20-50 GeV Jets)

Figure 7.17: The $K_{S}$ efficiency vs. $p_{T}$ curve for $K_{S}$ inside $20-50 \mathrm{GeV}$ jets. Each point in the top figure represents a $p_{T}$ value which implemented track-embedding. These points are divided into 2 distinct fit regions shown in the bottom diagrams. 


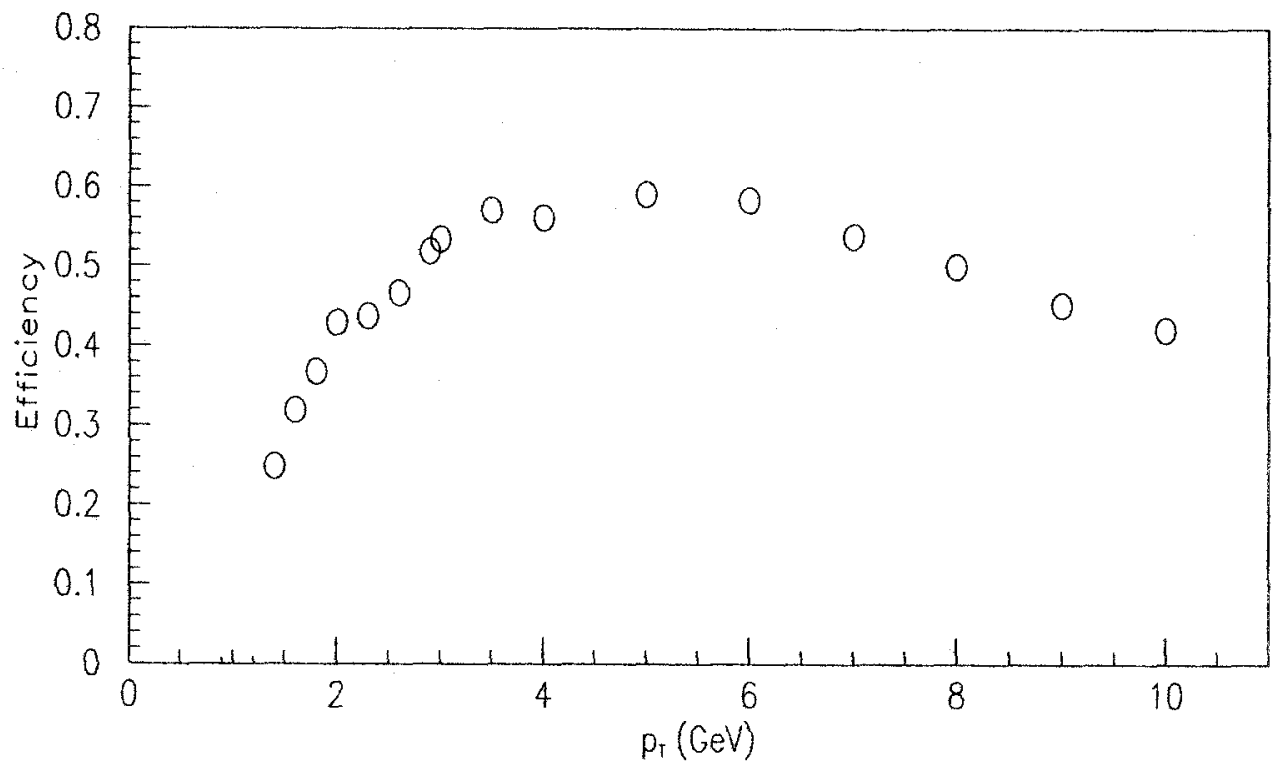

Efficiency vs. $\rho_{\mathrm{T}}$ (in 50-100 GeV Jets)

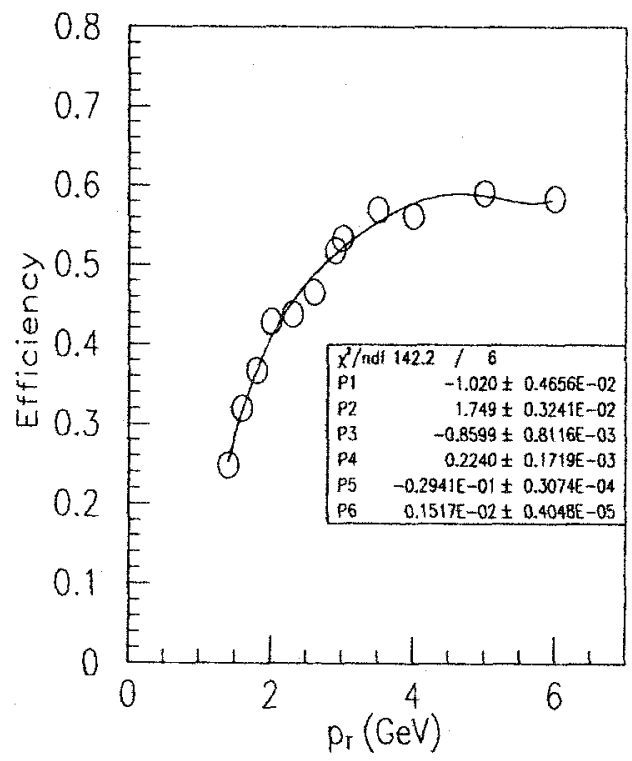

Efficiency vs. $p_{T}$ (in $50-100 \mathrm{GeV}$ Jets)

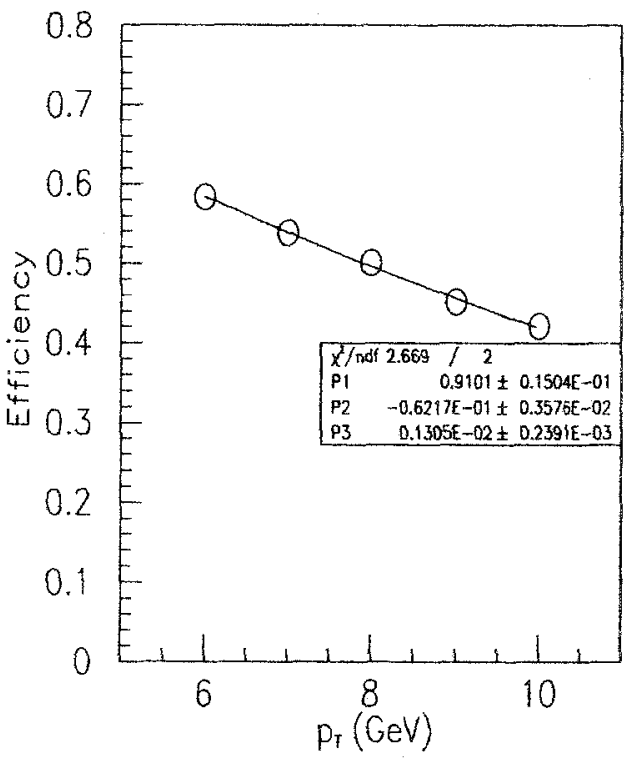

Efficiency vs. $p_{\mathrm{T}}$ (in $50-100 \mathrm{GeV}$ Jets)

Figure 7.18: The $K_{S}$ efficiency vs. $p_{T}$ curve for $K_{S}$ inside $50-100 \mathrm{GeV}$ jets. Each point in the top figure represents a $p_{T}$ value which implemented track-embedding. These points are divided into 2 distinct fit regions shown in the bottom diagrams. 


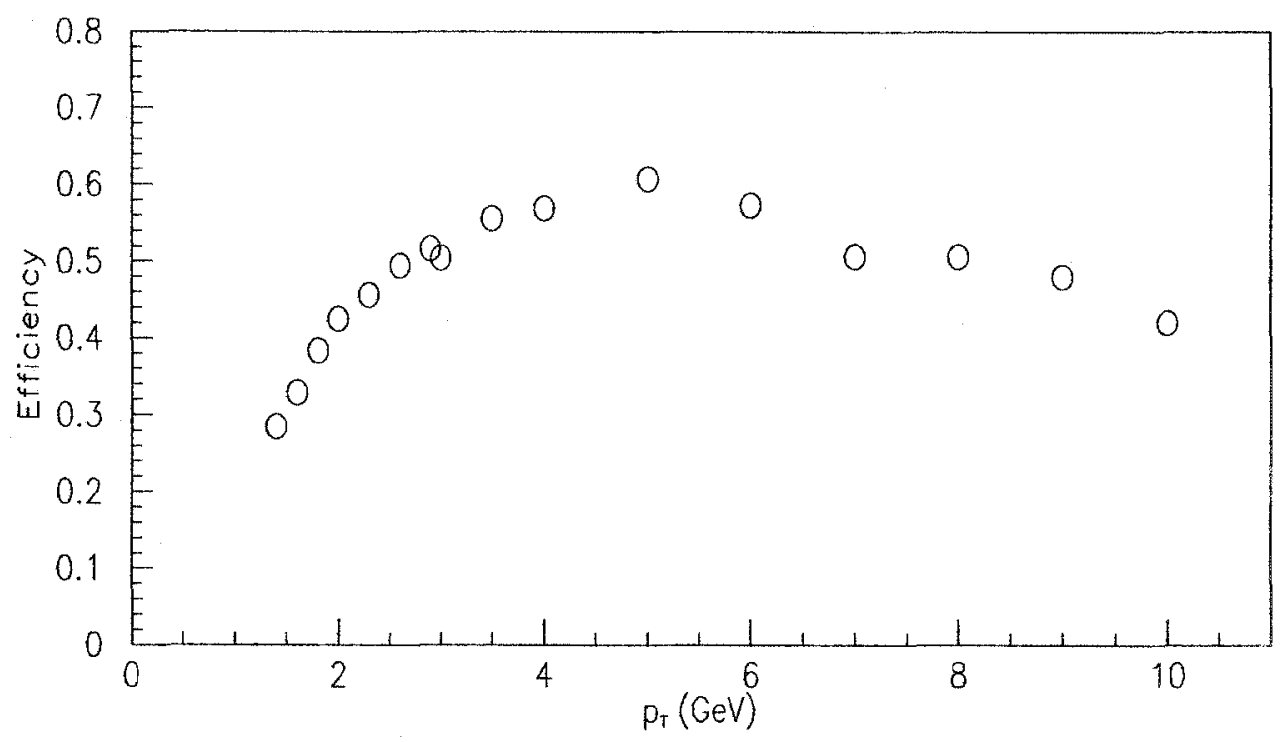

Efficiency vs. $p_{\mathrm{I}}$ (in $100-150 \mathrm{GeV}$ Jets)

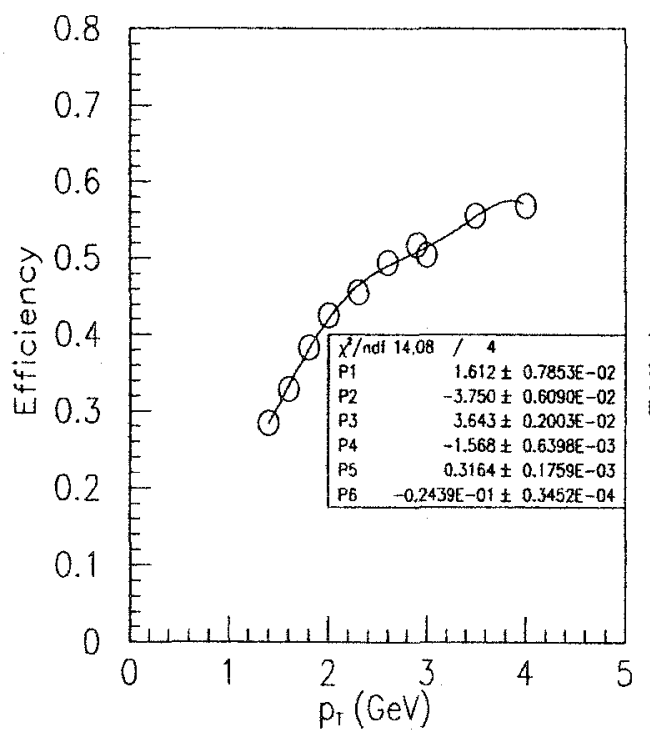

Efficiency vs. $p_{T}$ (in 100-150 GeV Jets)

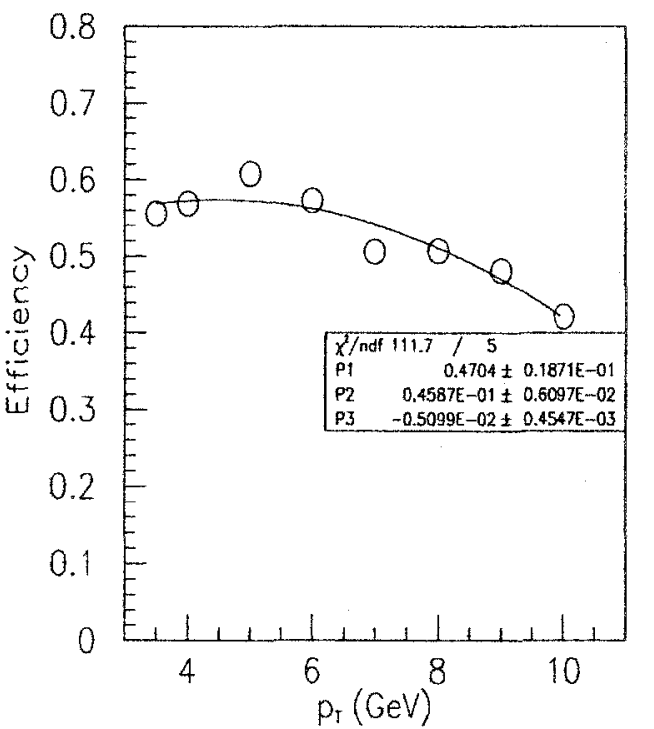

Efficiency vs. $p_{\mathrm{r}}$ (in 100-150 GeV Jets)

Figure 7.19: The $K_{S}$ efficiency vs. $p_{T}$ curve for $K_{S}$ inside $100-150 \mathrm{GeV}$ jets. Each point in the top figure represents a $p_{T}$ value which implemented track-embedding. These points are divided into 2 distinct fit regions shown in the bottom diagrams. 




Single Trock Efficiency vs. Pt (in 100-150 GeV Jets)

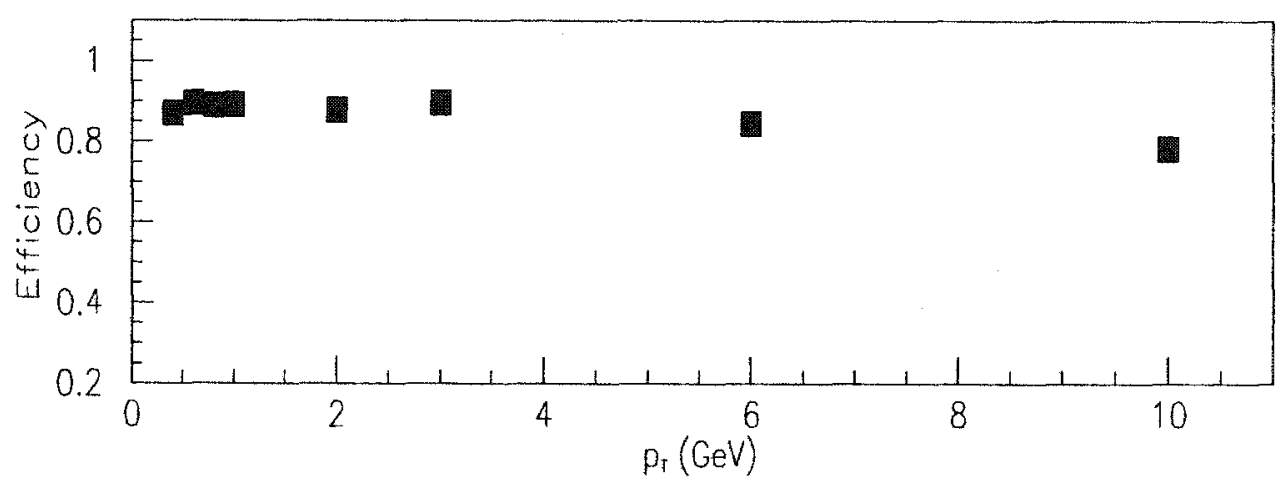

Single Track Efficiency vs. Pt (in 50-100 GeV Jets)

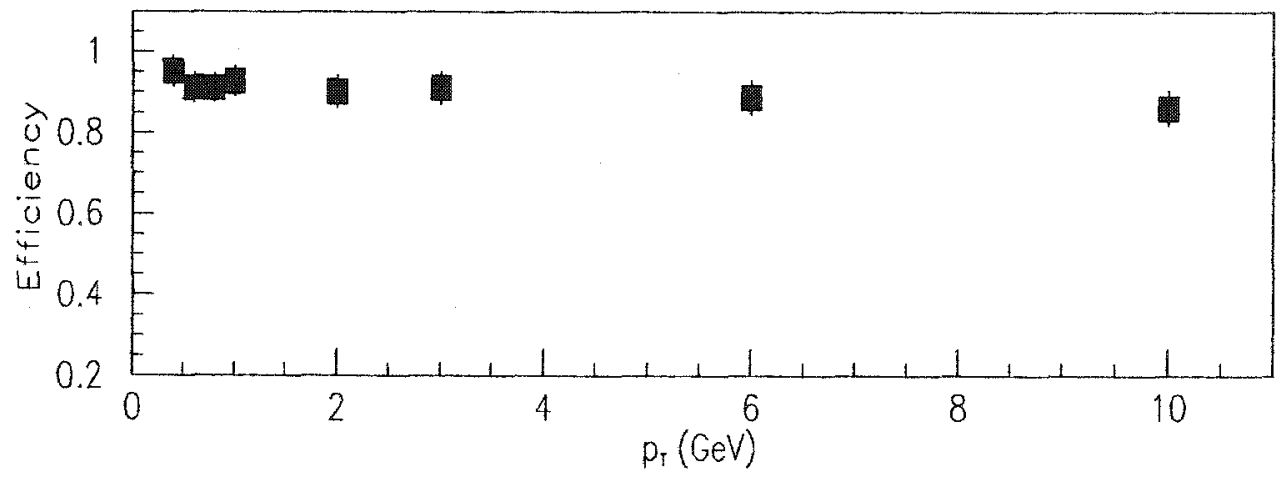

Single Track Efficiency vs. Pt (in 20-50 GeV Jets)

Figure 7.20: The single track efficiency vs. $p_{T}$ curve for single tracks inside 100$150 \mathrm{GeV}$ jets (top), $50-100 \mathrm{GeV}$ jets (middle), and $20-50 \mathrm{GeV}$ jets (bottom). Each point in the top figure represents a $p_{T}$ value which implemented track-embedding. 


\section{Chapter 8}

\section{Jets: Additional Corrections}

Aside from implementing the $p_{T}$ efficiency corrections, there are two additional adjustments necessary before arriving at any conclusions regarding the production of $K_{S}$ and tracks in jets. The first effect is due to the jet triggers which alter the jet $E_{T}$ spectrum and the number of tracks per jet, and the second factor is the inclusion of unwanted background $K_{S}$ originating from non-fragmentation processes. This chapter will discuss the trigger and background corrections applied to the jets, the tracks, and the $K_{S}$ in the data.

\subsection{Applying the $p_{T}$ Efficiency Corrections}

In regard to the types of physics plots generated in the analysis of $K_{S}$ and track production inside jets, the treatment will be substantially altered from the scenario of $K_{S}$ production described in Chapter 5. For the production of $K_{S}$ in the Minimum Bias events, quantities such as $\frac{d N_{K S}}{d \eta}$ and $\left\langle p_{T}\right\rangle$ are examined after fitting the $\frac{E}{N_{\text {event }}} \frac{d^{3} N_{K S}}{d^{3} p}$ spectra with a power law. These plots will be replaced in the jet study by the more relevant $\frac{1}{N_{j e t}} \frac{1}{p_{T}} \frac{d N_{K S}}{d p_{T}}$ and $\frac{1}{N_{j e t}} \frac{1}{p_{T}} \frac{d N_{\text {track }}}{d p_{T}}$ spectra. Furthermore, even though all of these plots from both the Minimum Bias and jet data originate from the $p_{T}$ curves corrected for the $p_{T}$ efficiencies, the way in which the $p_{T}$ spectra 
are generated and corrected for efficiencies will be quite different.

To generate the $\frac{E}{N_{\text {event }}} \frac{d^{3} N_{K S}}{d^{3} p}$ curve for Minimum Bias events, a single efficiencycorrected background-subtracted $p_{T}$ curve must be produced. To achieve this goal, the $K_{S}$ candidates passing the cuts (Chapter 3) are further divided into subsamples according to their mass. At this point, there are three $p_{T}$ spectra, one for each corresponding mass region. Following the background subtraction (Chapter 5), a single uncorrected background-subtracted $p_{T}$ curve for $K_{S}$ remains. Next, the $p_{T}$ efficiency corrections (Chapter 4) are applied bin-by-bin to this uncorrected background-subtracted $p_{T}$ curve to yield the efficiency corrected backgroundsubtracted $p_{T}$ spectra for $K_{S}$. Once this the spectra is computed, the $\frac{E}{N_{\text {event }}} \frac{d^{3} N_{K S}}{d^{3} p}$ spectrum is obtained by dividing the number of $K_{S}$ in each bin of the efficiency corrected $\frac{d N_{K S}}{d p_{T}}$ curve by the $p_{T}$ value at the midpoint of the bin, the $p_{T}$ bin width, the number of Minimum Bias events, and a constant of $8 \pi$. The generated $\frac{E}{N_{\text {event }}}$ $\frac{d^{3} N_{K S}}{d^{3} p} \quad$ spectra may now be fitted with a power law to compute $\frac{d N_{K S}}{d \eta}$ and $\left\langle p_{T}\right\rangle$ values.

Although the same techniques applied in the Minimum Bias study may be used again, another method is implemented. In particular, correcting for $p_{T}$ efficiencies will occur prior to background subtraction rather than after. These methods are equivalent. The latter method is preferred since other corrections will also be added in the form of weights. Also, since the particles contained within jets are measured with respect to the jet axis, producing the $\frac{E}{N_{\text {event }}} \frac{d^{3} N_{K S}}{d^{3} p} \quad$ spectra from the efficiencycorrected background subtracted $p_{T}$ curves is not the objective. This is the case because the $K_{S}$ (and tracks) inside jets are not distributed randomly in $\eta$ and $\phi$ space like the $K_{S}$ in the Minimum Bias data. Hence, the quantities used in Chapter 5 , like the $\frac{E}{N_{e v e n t}} \frac{d^{3} N_{K S}}{d^{3} p}$ and $\frac{d N_{K S}}{d \eta}$, are uninformative. Instead, the efficiency- 
corrected background-subtracted $p_{T}$ curves are converted into the $\frac{1}{N_{j e t}} \frac{1}{p_{T}} \frac{d N_{K S}}{d p_{T}}$ and $\frac{1}{N_{j e t}} \frac{1}{p_{T}} \frac{d N_{t r a c k}}{d p_{T}}$ spectra.

At this time, the computation of the $\frac{1}{N_{j e t}} \frac{1}{p_{T}} \frac{d N_{K S}}{d p_{T}}$ and $\frac{1}{N_{j e t}} \frac{1}{p_{T}} \frac{d N_{\text {track }}}{d p_{T}}$ curves with only the $p_{T}$ efficiency corrections will be described. For every $K_{S}$ candidate, there is associated along with it, a $p_{T}$ and jet $E_{T}$ value. Then, utilizing the efficiency curves determined in Chapter 7 , each $K_{S}$ and track candidate located inside a jet and passing all selection criteria in Chapter 6 is weighted by the corresponding $p_{T}$ efficiency correction factor. By grouping the $K_{S}$ inside jets into subsamples of different ranges of $p_{T}$, the number of $K_{S}$ candidates inside jets having a particular reconstructed mass value is weighted. Next, the series of weighted mass plots have the background subtracted out. In the end, the number of $K_{S}$ inside jets are tallied for a given interval of $p_{T}$, and they are plotted against $p_{T}$ to yield the efficiency corrected $p_{T}$ spectra. Similarly, the number of tracks inside jets with a particular $p_{T}$ are also weighted and grouped according to $p_{T}$. Now, the number of $K_{S}$ and tracks within each $p_{T}$ bin is divided by the $p_{T}$ value at the midpoint of the bin, the bin width, and the number of jets in a given jet $E_{T}$ range (20-50 GeV, 50-100 $\mathrm{GeV}$, or 100-150 GeV). In Figures 8.1 and 8.2 , the $\frac{1}{N_{j e t}} \frac{1}{p_{T}} \frac{d N_{K S}}{d p_{T}}$ and $\frac{1}{N_{j e t}} \frac{1}{p_{T}} \frac{d N_{\text {track }}}{d p_{T}}$ spectra are shown following the $p_{T}$ efficiency corrections for $K_{S}$ and tracks inside $20-50 \mathrm{GeV}, 50-100 \mathrm{GeV}$, and $100-150 \mathrm{GeV}$ jets. In addition, the " $p_{T}$ " notation in Tables 8.1-8.3 refer to values after the $p_{T}$ efficiency corrections have been applied. At this time, the other corrections will be discussed.

\subsection{Trigger Corrections to the Data}

The jet data sample consists of the combination of all unique events passing the JET20, JET50, JET70, and JET100 triggers described in Chapter 2. Only the 
effects of the jet triggers and the procedures followed to adjust the data will be outlined in this section. The origin as to why the triggers alter the data will not be studied in this analysis, but the jet triggers are known to be correlated to the jet $E_{T}$ [73] [74]. Thus, by weighting the jets, the tracks in the jets, and the $K_{S}$ in the jets by various functions of the jet $E_{T}$, both the jet $E_{T}$ and the number of tracks per jet trigger corrections are made.

\subsubsection{Jet $E_{T}$ Effect}

Since the production of $K_{S}$ and tracks in jets is a function of the jet $E_{T}$, the results should be calculated with respect to a particular jet $E_{T}$ spectrum. Although for the contrasting of the data with the Monte Carlo any jet $E_{T}$ spectrum would suffice, it is best to incorporate the shape of the jet $E_{T}$ cross-section curve as measured by CDF for the presentation of the results [73] [75] [76]. Hence, the effects of the trigger on the jet $E_{T}$ will be undone by weighting the jet $E_{T}$ spectra in the data and the Monte Carlo (to be discussed later) to be the same shape as the jet $E_{T}$ cross-section. Furthermore, the $K_{S}$ and tracks in jets are weighted along with the jets in order to undo the jet $E_{T}$ effect of the trigger.

The jet data sample (JET 20, JET50, JET70, and JET100 sets) is ran through, and the jet $E_{T}$ spectra for the $20-50 \mathrm{GeV}, 50-100 \mathrm{GeV}$, and $100-150 \mathrm{GeV}$ jets is histogramed in Figure 8.3. The bizarre jet $E_{T}$ spectrum in Figure 8.3 shows the various peaks and dips caused by the combined effect of all the jet triggers of each set. The uniqueness of the jets is ensured by the removal of the events included in more than one data set as described in Chapter 6. Even though the distributions of the jet $E_{T}$ vary upon the jet trigger data set, these effects can be corrected for all sets simultaneously. 
Before adjusting the jet $E_{T}$ histogram to be identical to the shape of the jet $E_{T}$ cross-section, the jet $E_{T}$ spectra is weighted to be flat. In Figure 8.4, all three jet energy ranges are weighted in this manner. The $20-50 \mathrm{GeV}$ case has a pedestal equal to 30,000 and the $50-100$ and $100-150 \mathrm{GeV}$ cases have pedestals of 40,000 . Any constant would do as long as it is fixed because the number of jets is divided out before particular quantities are computed. For the fragmentation measurement, it is better to quote results using the flat jet $E_{T}$ spectra. In addition, $K_{S}$ and tracks are weighted with the same values as the jets they are contained within.

This flat distribution is implemented because some other experiments have finely tuned $e^{+} e^{-}$collisions at a certain energy. This scenario is unlike the $p \bar{p}$ collisions at the Tevatron where the individual interacting quarks and gluons contain an arbitrary fraction of the energy of the proton and antiproton. The $e^{+} e^{-}$collisions give rise to back-to-back jets having energies on the order of $\frac{\sqrt{s}}{2}$. So when contrasting fragmentation results with other experiments, [9] [77] [78] [79] [80] [81], the jet energies produced from $e^{+} e^{-}$collisions are considered to be approximately constant.

For the $\frac{1}{N_{j e t}} \frac{1}{p_{T}} \frac{d N_{K S}}{d p_{T}}$ and $\frac{1}{N_{j e t}} \frac{1}{p_{T}} \frac{d N_{\text {track }}}{d p_{T}}$ as well as the number of $K_{S}$ per jet and the number of tracks per jet calculations, the jet $E_{T}$ is weighted so the resulting spectrum is similar to the shape of the jet cross-section as a function of jet $E_{T}$. This is accomplished by first obtaining the flat jet $E_{T}$ distribution, and then weighting this spectrum in order to reproduce the structure of the jet cross-section as a function of jet $E_{T}$. In Figure 8.5, the jet $E_{T}$ spectrum is shown after being corrected with this method. Again, the $K_{S}$ and tracks inside jets are weighted according to the value of the jet $E_{T}$ of the jet they are inside. The weighting of the Monte Carlo jets will be discussed later. 


\subsubsection{The Number of Tracks per Jet Effect}

The number of tracks per jet is also effected by the jet trigger. For each jet trigger, the number of tracks per jet is approximately $10 \%$ too high around and below the trigger threshold. Since the number of tracks per jet is dependent upon the onset of the jet trigger, it follows that the number of tracks per jet is a function of jet $E_{T}$. Moreover, the $K_{S}$, by decaying into charged pions, behave similarly to the tracks and consequently may be corrected using the identical approach.

The number of tracks per jet as a function jet $E_{T}$ is studied for each jet trigger sample separately. The examination of the jet trigger samples individually is different from the previous subsection where the jet $E_{T}$ trigger correction is carried out simultaneously to a sample consisting of all the jet triggers. For each trigger set, a given track is tallied as being contained within a jet if the following criteria are satisfied:

- Track Cuts

$$
\begin{aligned}
& -\left|z_{\text {primary }}\right|<60.0 \mathrm{~cm} \\
& -z_{j e t}^{\text {rms }}<5.0 \mathrm{~cm} \\
& -\left|z_{\text {primary }}-z_{\text {jet }}\right|<5.0 \mathrm{~cm} \\
& -\left|\eta^{\text {jet }}\right|<1.0 \\
& - \text { Tracks nearest } \Delta R=0.7 \text { Jet } \\
& -\left|z_{\text {primary }}-z_{\text {track }}\right|<5.0 \mathrm{~cm} \\
& -\left|\eta^{\text {track }}\right|<1.0 \\
& \text { - Saved According to jet } E_{T}
\end{aligned}
$$


Since the shape of the distribution is of concern, the properties that would alter the area of the distribution without changing the area normalized to unity are of little interest. The $p_{T}$ track and the $\left|z_{\text {primary }}-z_{\text {track }}\right|$ cuts, the track background, and the track efficiencies falling under this category are irrelevant here.

Without the jet triggers, the number of tracks per jet is expected to be a smoothly increasing function of jet $E_{T}$. In Figure 8.6, the number of tracks per jet vs. the jet $E_{T}$ is shown for each jet trigger set, and the data from each jet trigger set is denoted with the square symbols. Notice the bumps in around $50 \mathrm{GeV}$, 70-100 GeV, and 50-100 GeV for the JET50, JET70, and JET100 sets caused by the jet triggers. There is actually another such bump in the JET20 around $20-30 \mathrm{GeV}$ but it is hardly noticeable using the current divisions. If left uncorrected, below the onset on the jet trigger, the number of $K_{S}$ per jet and the number of tracks per jet would be approximately $10 \%$ too high.

Before adjusting the effect, the number of tracks per jet as a function of the jet $E_{T}$ must be studied with a sample that lacks a jet $E_{T}$ trigger. In this case, the muon trigger sample is selected. Either the electron or the photon data sets would have been sufficient; however, muons leave very little energy inside the calorimeter, unlike photons and electrons, and consequently the jets in the muon trigger sample would not require the removal of unwanted clusters prior to the calculation of jet $E_{T}$. Events passing both the muon trigger as well as any other jet trigger (JET20, JET50, JET70, and JET100) are excluded. In Figure 8.6, the number of tracks per jet vs. jet $E_{T}$ for the muon set, devoid of any jet trigger dependence, are denoted with circles and are fitted with a 4th order polynomial.

By dividing each jet trigger result by those from the muon trigger, the corrections are made to every jet trigger set separately for a given jet $E_{T}$ range. Hence, when 
the data is rerun, each track with a given jet $E_{T}$ is weighted to yield a smoothly increasing curve of the number of tracks per jet vs. jet $E_{T}$ spectrum. The identical weights are also applied to the $K_{S}$ in jets. Following the procedure just discussed, the trigger dependencies of the number of tracks per jet and the number $K_{S}$ per jet are decoupled from the sample. Thus, the number of tracks per jet and the number $K_{S}$ per jet are reduced by about $10 \%$ below the trigger threshold.

Other studies do not correct for the trigger effect on the number of tracks per jet in this manner. For example, in the inclusive cross-section study, [73] [74] [75] [76], jet $E_{T}$ thresholds of 75,100 , and $130 \mathrm{GeV}$ are applied to the 50,70 , and 100 $\mathrm{GeV}$ trigger samples in order to have a trigger efficiency above $95 \%$. In the dijet analyses, [82] [83] [28], the dijet masses are also selected to well above the 2.0 times the trigger thresholds. For another study of underlying events in low energy samples, [84] [85] [86], the $40 \mathrm{GeV}$ threshold is placed on the leading jet for the jet 20 trigger sample. This method does indeed cure the trigger problem, but many jets are lost.

The jet $E_{T}$ and the number of tracks per jet trigger corrections are simultaneously implemented on the jets, the tracks in jets, and the $K_{S}$ in jets as a product of two weights which are functions of jet $E_{T}$. On the other hand, the jets are only adjusted for the jet $E_{T}$ trigger effect. In the next section, the subtraction of the background $K_{S}$ and tracks from the number of the $K_{S}$ and tracks inside jets will be described.

\subsection{Background Corrections to the Data}

Not all of the $K_{S}$ and tracks inside jets originate from fragmentation, some are produced by other low energy processes. The background $K_{S}$ and tracks in jets 
are considered to be "fake" since they are of no interest in this analysis. Though the particles are indeed real $K_{S}$ and tracks, these are not the $K_{S}$ and tracks in jets being sought out. These "fake" $K_{S}$ and tracks in jets need to be subtracted from the total number of $K_{S}$ and tracks in jets in order to prevent the resulting quantities from being at most $8 \%$ too high. This problem is dealt with by calculating how many $K_{S}$ and tracks are in a random "fake" cone away from all other jets, and then subtracting this quantity from the number of $K_{S}$ and tracks found in jets after trigger corrections are performed.

There are many differences between a "fake" jet and a "real" jet. A "real" jet is defined by the clustering algorithms and the jet energy corrections mentioned in Chapter 6. Also, for a "real" jet, the $\eta_{j e t}$, the $\phi_{j e t}$, the jet $E_{T}$, and the jet vertex values are ultimately computed with the distributions of particles in a given event. In contrast, a "fake" jet does not exist in any material sense. A "fake" jet is not derived from the particles in the clusters found in the calorimeters, but rather via Monte Carlo techniques. Having just an $\eta_{\text {jet }}^{\text {fake }}$ and a $\phi_{\text {jet }}^{\text {fake }}$, a "fake" jet only exists if it is sufficiently isolated from all "real" jets. Moreover, a "fake" jet, not being calculated from particles, does not have either a jet $E_{T}$ or a jet vertex. Apart from this, since there is not a jet $E_{T}$ associated with this "fake" jet, the trigger corrections (jet $E_{T}$ and the number of tracks per jet) are not made. A "fake" jet can be thought as an arbitrary cone away from the other jets in which the $K_{S}$ and the tracks may be inside.

In order to determine whether either a track or a $K_{S}$ is contained within a "fake jet", the direction of a "fake" jet is defined by a random $\eta_{j e t}^{\text {fake }}\left(\left|\eta_{\text {jet }}^{\text {fake }}\right|<1.0\right)$ and a random $\phi_{j e t}^{f a k e}\left(0 \leq \phi_{j e t}^{f a k e}<2 \pi\right)$. The $\eta_{j e t}^{f a k e}$ should also have the same shape as the $\eta_{j e t}$ distribution of the "real" jets from the data. The selection of random $\eta_{j e t}^{\text {fake }}$ and 
$\phi_{\text {jet }}^{\text {fake }}$ values is repeated until $\Delta R_{\text {jet-fake jet }}>1.0$ for every "real" jet above $10.0 \mathrm{GeV}$ where $\Delta R_{\text {jet-fake jet }}$ is given by:

$$
\Delta R_{\text {jet-fake jet }}=\sqrt{\left(\eta_{j e t}-\eta_{j e t}^{f a k e}\right)^{2}+\left(\phi_{j e t}-\phi_{j e t}^{f a k e}\right)^{2}}
$$

Once this iterative process of locating a "fake" jet direction sufficiently away from all other "real" jets above $10.0 \mathrm{GeV}$ is complete, then the number of $K_{S}$ and tracks within the "fake" jet is computed. In particular, if there is a $K_{S}$ so $\Delta R_{K S-f a k e ~ j e t}<0.7$ where $\Delta R_{K S-f a k e ~ j e t}$ is denoted by

$$
\Delta R_{K S-f a k e ~ j e t}=\sqrt{\left(\eta_{K S}-\eta_{j e t}^{f a k e}\right)^{2}+\left(\phi_{K S}-\phi_{j e t}^{f a k e}\right)^{2}}
$$

, then the $K_{S}$ is considered a background $K_{S}$. Equivalently, a track is tallied as a background track if $\Delta R_{\text {track-fake jet }}<0.7$ where $\Delta R_{\text {track-fake jet }}$ is defined by

$$
\Delta R_{\text {track-fake jet }}=\sqrt{\left(\eta_{\text {track }}-\eta_{\text {jet }}^{\text {fake }}\right)^{2}+\left(\phi_{\text {track }}-\phi_{\text {jet }}^{\text {fake }}\right)^{2}}
$$

Both $K_{S}$ and tracks found inside "fake" jets should pass some of the cuts outlined in Chapter 6. Associating "fake" jets into $E_{T}$ ranges or cutting on jet vertices is not possible since the "fake" jet is defined only by $\eta_{j e t}^{\text {fake }}$ and $\phi_{j e t}^{f a k e}$. However, most other cuts are utilized.

Once the $p_{T}$ spectra for background $K_{S}$ and tracks are obtained, the curves derived from $K_{S}$ and tracks embedded inside $20-50 \mathrm{GeV}$ jets are implemented in order to correct for efficiencies. This is done because the "fake" jets are believed not to contain many tracks. Since the $20-50 \mathrm{GeV}$ have lower track multiplicities than either $50-100 \mathrm{GeV}$ or $100-150 \mathrm{GeV}$ jets, the track-embedding efficiency curves 
from Chapter 7 obtained from $K_{S}$ and tracks embedded into $20-50 \mathrm{GeV}$ jets are incorporated.

In summary, the selection criteria of "fake" jets as well as for the background tracks and $K_{S}$ inside the "fake" jets are listed below:

- Fake Jet Cuts

$-\left|z_{\text {primary }}\right|<60.0 \mathrm{~cm}$

- Select $\eta_{\text {jet }}^{\text {fake }}$ with $\left|\eta_{\text {jet }}^{\text {fake }}\right|<1.0$

- Select $\phi_{j e t}^{f a k e}$ with $0 \leq \phi_{j e t}^{f a k e}<2 \pi$

- For all jets with $E_{T}>10.0 \mathrm{GeV},\left|\Delta R_{\text {jet-fake jet }}\right|>1.0$

- Track Cuts

$-\left|z_{\text {primary }}\right|<60.0 \mathrm{~cm}$

$-\Delta R_{\text {track-fake jet }}<0.7$

$-\left|\eta^{\text {track }}\right|<1.0$

$-1.5<p_{T}^{\text {track }}<10.0 \mathrm{GeV}$

- Correct with $p_{T}$ efficiency curve from tracks embedded into $20-50 \mathrm{GeV}$ jets

- $K_{S}$ Cuts

$-\left|z_{\text {primary }}\right|<60.0 \mathrm{~cm}$

$-\chi_{K S}^{2}<20.0$

$-\left|z_{K S}-z_{\text {primary }}\right|<3.0 \mathrm{~cm}$

- $p_{T}^{\text {tracks from } K S}>500 \mathrm{MeV}$ 
$-\left|\eta^{K S}\right|<1.0$

- 3D Displacement ${ }^{K S}>1.0 \mathrm{~cm}$

$-\cos \theta_{P D}^{K S}>0.990$

$-\Delta R_{K S-\text { fake jet }}<0.7$

$-1.5<p_{T}^{K S}<10.0 \mathrm{GeV}$

- Correct with $p_{T}$ efficiency curve from $K_{S}$ embedded into $20-50 \mathrm{GeV}$ jets

The $\frac{1}{N_{j e t}} \frac{1}{p_{T}} \frac{d N_{K S}}{d p_{T}}$ and $\frac{1}{N_{j e t}} \frac{1}{p_{T}} \frac{d N_{\text {tracks }}}{d p_{T}}$ spectra for background $K_{S}$ and tracks as a function of $p_{T}$ are displayed in Figures 8.7 and 8.8. Although the HERWIG Monte Carlo results will be detailed in Chapter 9, it is shown along with the data. The square points represent the data whereas the three histograms generated with the HERWIG Monte Carlo correspond to three different jet $E_{T}$ range settings. Both data and HERWIG Monte Carlo curves are computed from the $K_{S}$ and tracks located within the "fake" jets. Notice that the data is higher than the HERWIG Monte Carlo (discussed in Chapter 9). The background curves are later subtracted bin-by-bin from the $p_{T}$ spectra of $K_{S}$ and tracks inside jets. Most of these background corrections are in low $p_{T}$ regions.

In the end, after the trigger adjustments, the background rate of $K_{S}$ and tracks in the data is at most an $8 \%$ effect. To be specific, of $2,078,760$ fake jets, there are $25979 K_{S}$ between 1.5 and $10 \mathrm{GeV}$ once the $p_{T}$ and the branching ratio corrections are made. The background rate of $K_{S}$ is $0.0125 \pm 0.0003$. Likewise, in the same ensemble of "fake" jets, there are 465099 tracks between 1.5 and $10 \mathrm{GeV}$ after the single track $p_{T}$ efficiency adjustments. This corresponds to a track background of $0.2237 \pm 0.0003$ tracks per jet. 


\subsection{Results from the Data}

In Tables 8.1 through 8.3 , the effect of the various corrections for the number of $K_{S}$ per jet, the number of tracks per jet, and 2.0 times the number of $K_{S}$ per track for data are listed for the $K_{S}$ and tracks inside $20-50 \mathrm{GeV}, 50-100 \mathrm{GeV}$, and 100-150 $\mathrm{GeV}$ jets. For example, in Table 8.1, the number of $K_{S}$ per jet in $20-50 \mathrm{GeV}$ jets is $0.175 \pm 0.002$. This number is taken after the branching ratio, the $p_{T}$ efficiency, and the jet $E_{T}$ trigger corrections have been taken into account. After the number of tracks per jet trigger adjustment, this number decreases to $0.168 \pm 0.002$. Moreover, after the background (or "fake") $K_{S}$ have been removed, the number of $K_{S}$ per jet is reduced to $0.156 \pm 0.002$. The number of tracks per jet and 2.0 times the number of $K_{S}$ per track may be understood similarly.

The motivation for multiplying the number of $K_{S}$ per track by 2.0 is to take into account $K_{L}$ production. It is assumed that the sum of $K_{S}$ and $K_{L}$ are equal, so $2.0^{*} K_{S}$ production is equal to $K_{S}$ plus $K_{L}$ production. Now, the sum of $K_{S}$ and $K_{L}$ production $\left(2.0 K_{S}\right.$ production) is equal to both the sum of $K_{0}$ and $\bar{K}_{0}$ production and the sum of $K^{+}$and $K^{-}$production. It turns out that the ratio of the charged kaons to the charged pions is about $10 \%$ for Minimum Bias events. So in order to study this ratio inside high $E_{T}$ jets, it becomes necessary to multiply $K_{S}$ production by 2.0 and dividing this product by the number of tracks (approximately the number of charged pions).

From viewing Tables 8.1 through 8.3, various trends are observable. In general, the number of tracks per jet trigger correction has the largest effect on the number of $K_{S}$ per jet and the number of tracks per jet below $100 \mathrm{GeV}$ because most of the jet sample comes from the Jet100 set (mainly the jets below the threshold are effected). As for the background rate, every energy range is taken into account 
Table 8.1: The number of $K_{S}$ per jet in the data $\left(1.5<p_{T}<10.0 \mathrm{GeV}\right)$.

\begin{tabular}{|c|c|c|c|}
\hline $\begin{array}{c}\text { After } \\
\text { Correction }\end{array}$ & $\begin{array}{c}\frac{N_{K S}}{j e t} \\
\text { in } 20-50 \text { Jets }\end{array}$ & $\begin{array}{c}\frac{N_{K S}}{j e t} \\
\text { in } 50-100 \text { Jets }\end{array}$ & $\begin{array}{c}\frac{N_{K S}}{j e t} \\
\text { in 100-150 Jets }\end{array}$ \\
\hline$P_{T}$ & $0.175 \pm 0.002$ & $0.232 \pm 0.003$ & $0.218 \pm 0.003$ \\
Trigger & $0.168 \pm 0.002$ & $0.219 \pm 0.003$ & $0.213 \pm 0.003$ \\
Fake Rate & $0.156 \pm 0.002$ & $0.206 \pm 0.003$ & $0.200 \pm 0.003$ \\
\hline
\end{tabular}

Table 8.2: The number of tracks per jet in the data $\left(1.5<p_{T}<10.0 \mathrm{GeV}\right)$.

\begin{tabular}{|c|c|c|c|}
\hline $\begin{array}{c}\text { After } \\
\text { Correction }\end{array}$ & $\begin{array}{c}\frac{N_{\text {track }}}{j e t} \\
\text { in 20-50 Jets }\end{array}$ & $\begin{array}{c}\frac{N_{\text {track }}}{\text { jet }} \\
\text { in } 50-100 \text { Jets }\end{array}$ & $\begin{array}{c}\frac{N_{\text {track }}}{\text { jet }} \\
\text { in 100-150 Jets }\end{array}$ \\
\hline$P_{T}$ & $3.095 \pm 0.002$ & $5.415 \pm 0.003$ & $6.282 \pm 0.003$ \\
Trigger & $3.026 \pm 0.002$ & $5.314 \pm 0.003$ & $6.181 \pm 0.003$ \\
Fake Rate & $2.802 \pm 0.002$ & $5.090 \pm 0.003$ & $5.957 \pm 0.003$ \\
\hline
\end{tabular}

Table 8.3: The number of $\left(2.0^{*} K_{S}\right)$ per track in the data $\left(1.5<p_{T}<10.0 \mathrm{GeV}\right)$.

\begin{tabular}{|c|c|c|c|}
\hline $\begin{array}{c}\text { After } \\
\text { Correction }\end{array}$ & $\begin{array}{c}2 \frac{N_{K S}}{N_{\text {track }}} \\
\text { in } 20-50 \text { Jets }\end{array}$ & $\begin{array}{c}2 \frac{N_{K S}}{N_{\text {track }}} \\
\text { in } 50-100 \text { Jets }\end{array}$ & $\begin{array}{c}2 \frac{N_{K S}}{N_{\text {track }}} \\
\text { in } 100-150 \text { Jets }\end{array}$ \\
\hline$P_{T}$ & $0.113 \pm 0.001$ & $0.086 \pm 0.001$ & $0.069 \pm 0.001$ \\
Trigger & $0.111 \pm 0.001$ & $0.082 \pm 0.001$ & $0.069 \pm 0.001$ \\
Fake Rate & $0.111 \pm 0.001$ & $0.081 \pm 0.001$ & $0.067 \pm 0.001$ \\
\hline
\end{tabular}


following this procedure. For the data, after all the corrections, the number of $K_{S}$ per jet increases from $0.156 \pm 0.002$ for $K_{S}$ inside $20-50 \mathrm{GeV}$ jets to $0.200 \pm 0.003$ for $K_{S}$ inside $100-150 \mathrm{GeV}$ jets. Hence, the number of $K_{S}$ per jet increases by about $30 \%$. The number of tracks per jet increases by more than a factor of 2.1 from $2.802 \pm 0.002$ to $5.957 \pm 0.003$ over a similar range. As for 2.0 times the number of $K_{S}$ per track, this quantity decreases by approximately a factor of 0.60 from $0.111 \pm 0.001$ to $0.067 \pm 0.001$. In Chapter 9 and Chapter 10, the results from the data will be compared with those from the HERWIG Monte Carlo. 


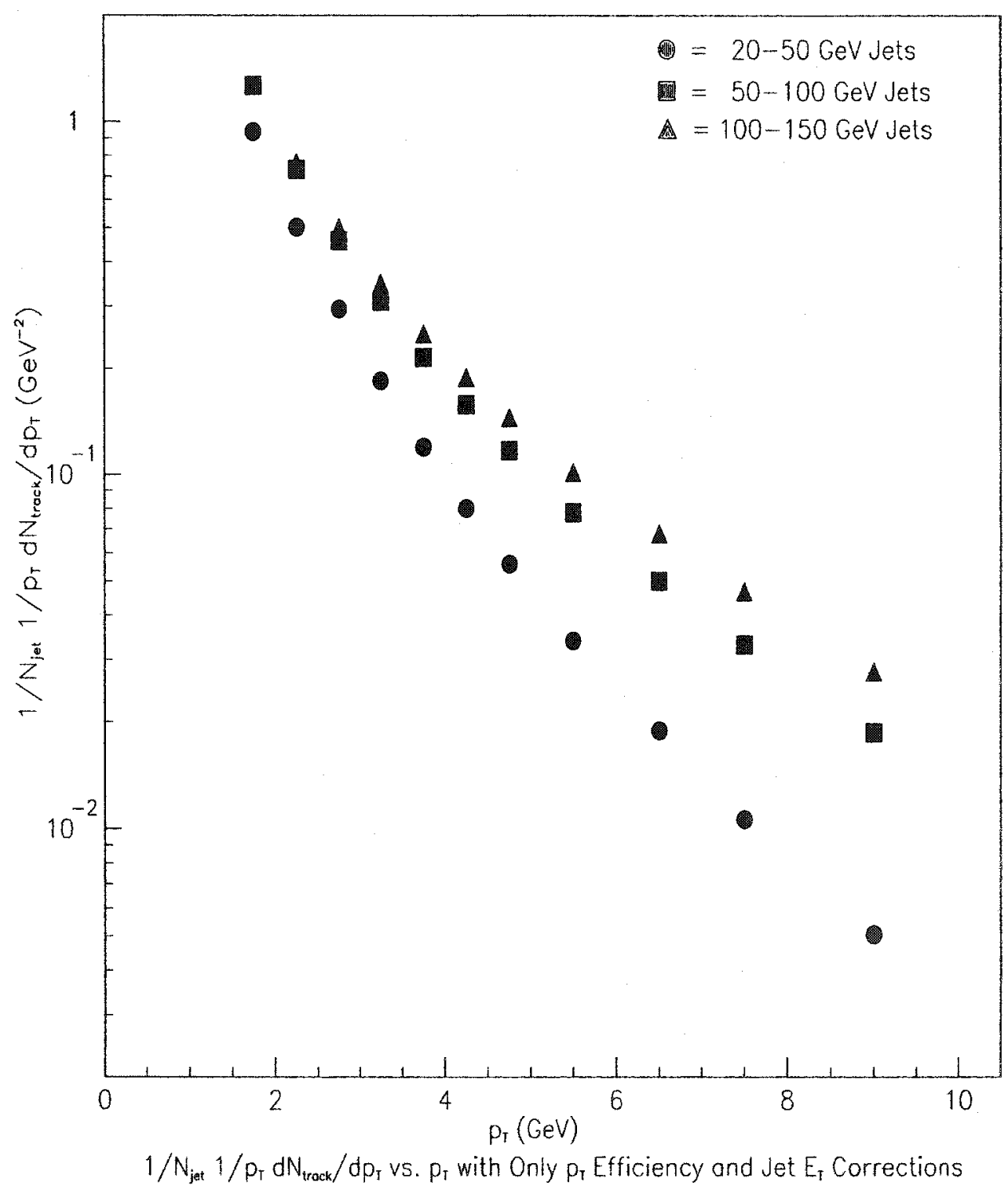

Figure 8.1: The $\frac{1}{N_{j e t}} \frac{1}{p_{T}} \frac{d N_{K S}}{d p_{T}}$ spectra with only $p_{T}$ efficiency and jet $E_{T}$ trigger corrections for $20-50 \mathrm{GeV}, 50-100 \mathrm{GeV}$, and $100-150 \mathrm{GeV}$ jets. 


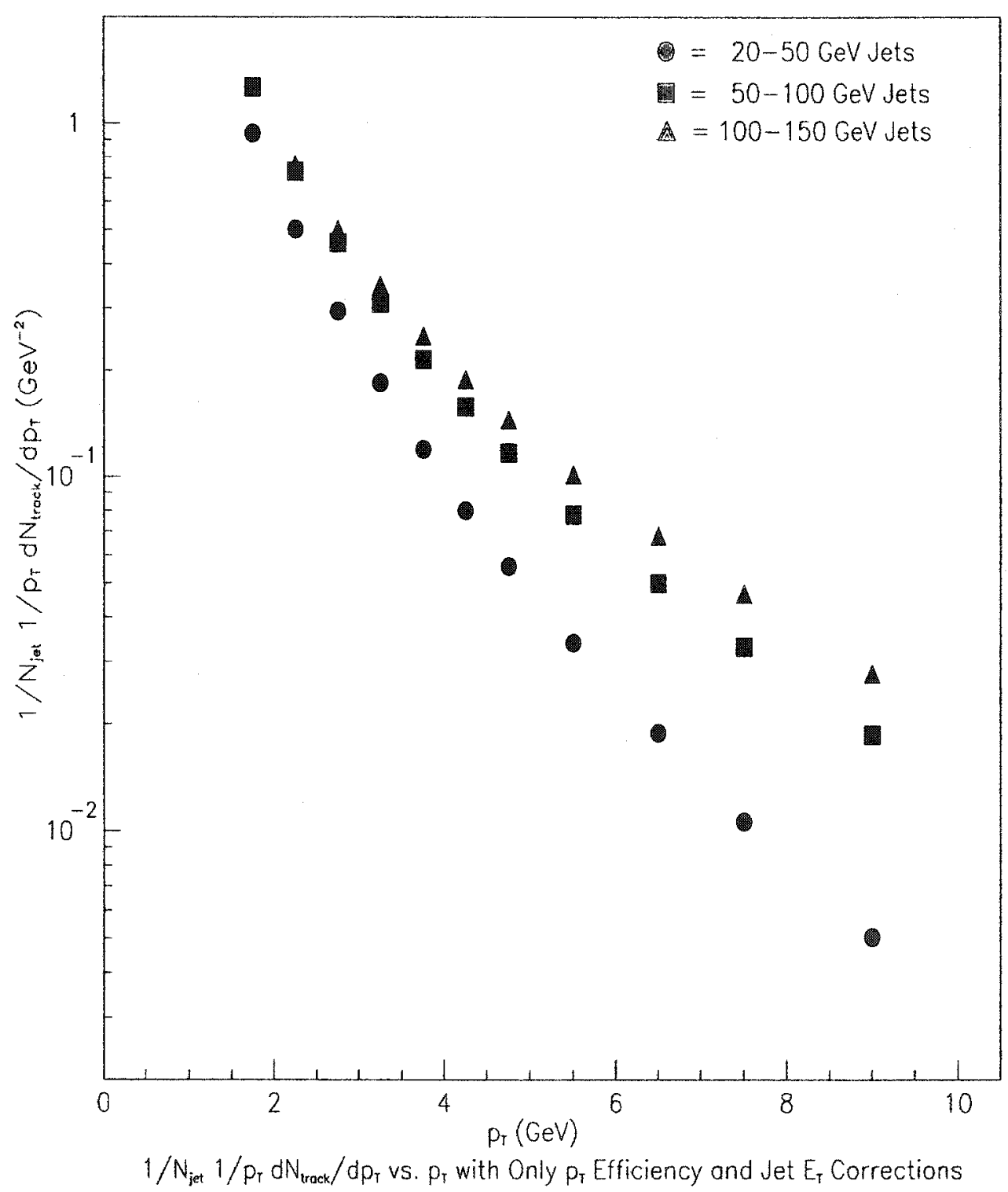

Figure 8.2: The $\frac{1}{N_{j e t}} \frac{1}{p_{T}} \frac{d N_{\text {track }}}{d p_{T}}$ spectra with only $p_{T}$ efficiency and jet $E_{T}$ trigger corrections for $20-50 \mathrm{GeV}, 50-100 \mathrm{GeV}$, and $100-150 \mathrm{GeV}$ jets. 


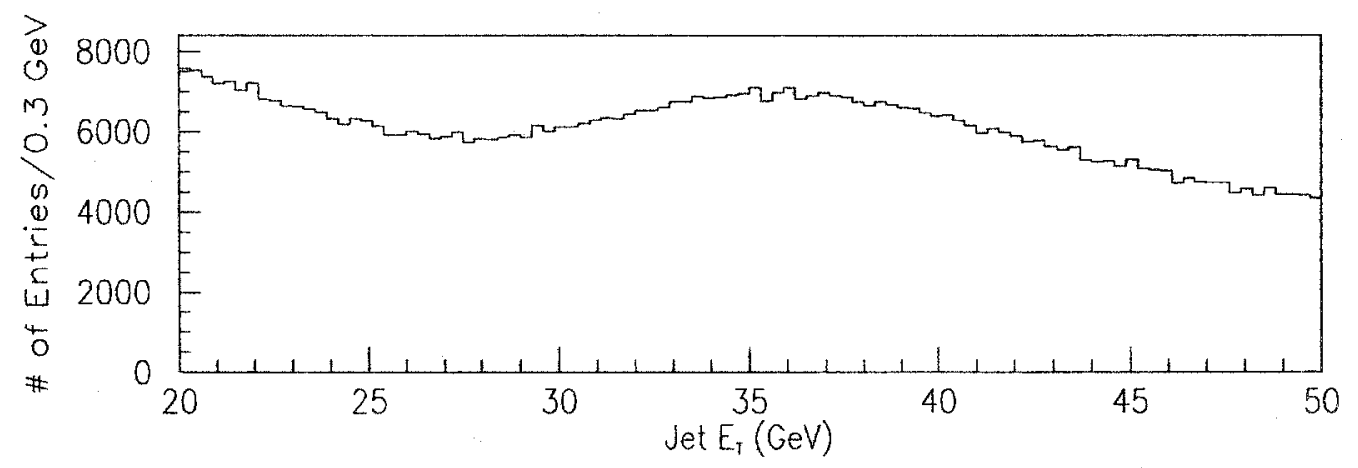

Jet $E_{T}$ (Only Corrected for Tracks per Jet Trigger Effect)



Jet $E_{T}$ (Only Corrected for Tracks per Jet Trigger Effect)



Jet $E_{\mathrm{T}}$ (Only Corrected for Tracks per Jet Trigger Effect)

Figure 8.3: The unweighted jet $E_{T}$ for $20-50 \mathrm{GeV}$ (top), $50-100 \mathrm{GeV}$ (middle), and $100-150 \mathrm{GeV}$ (bottom) jets. For all jet $E_{T}$ ranges, the jet $E_{T}$ spectrum has not yet been corrected for the jet $E_{T}$ dependence. Only the number of tracks per jet trigger effect has been taken into account. 


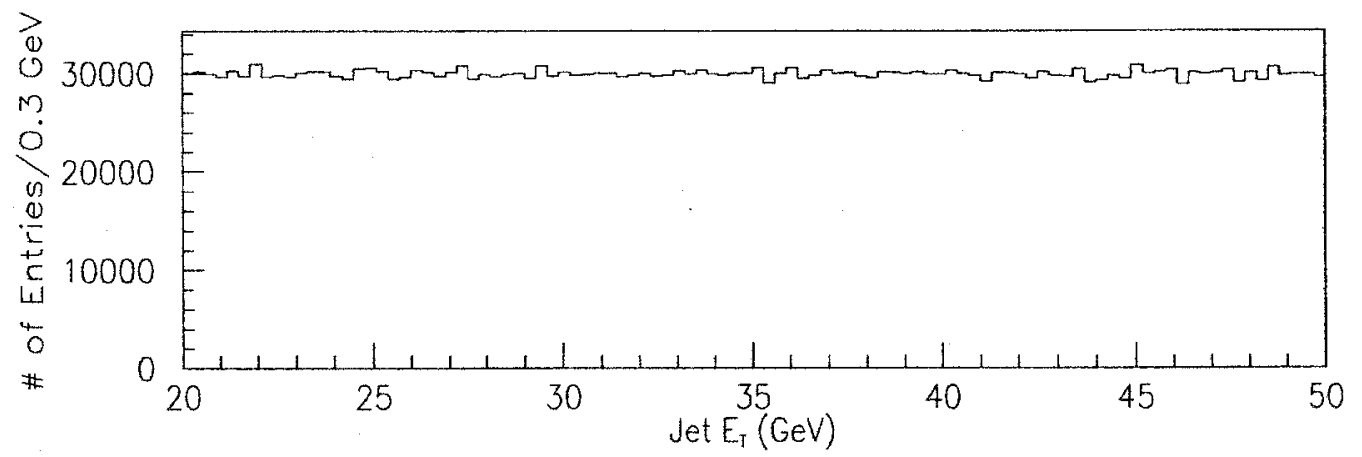

Jet $E_{\mathrm{T}}$ (Weighted by Flat Distribution)

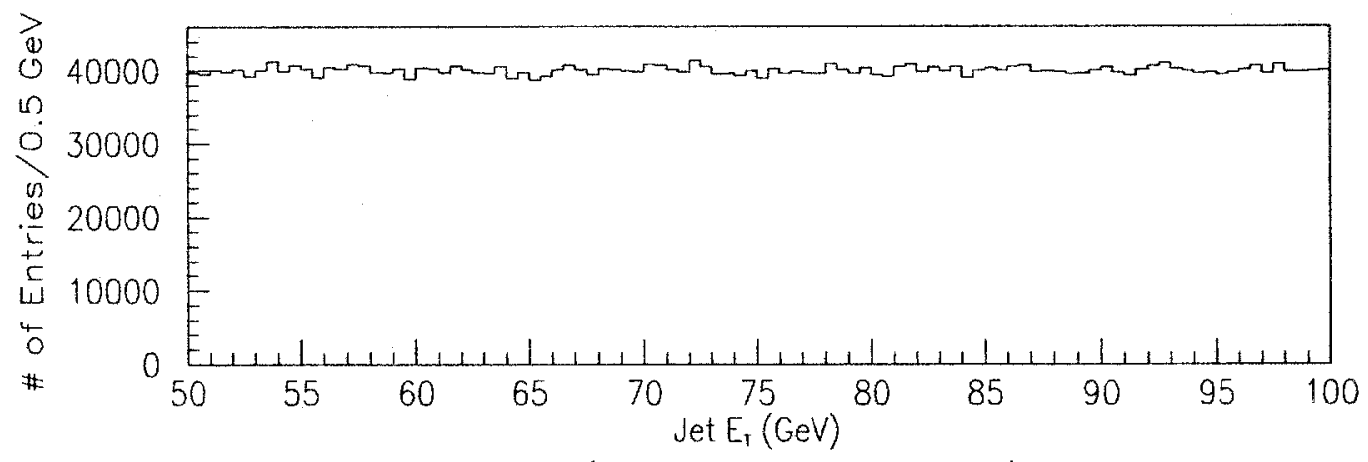

Jet $E_{T}$ (Weighted by Flat Distribution)

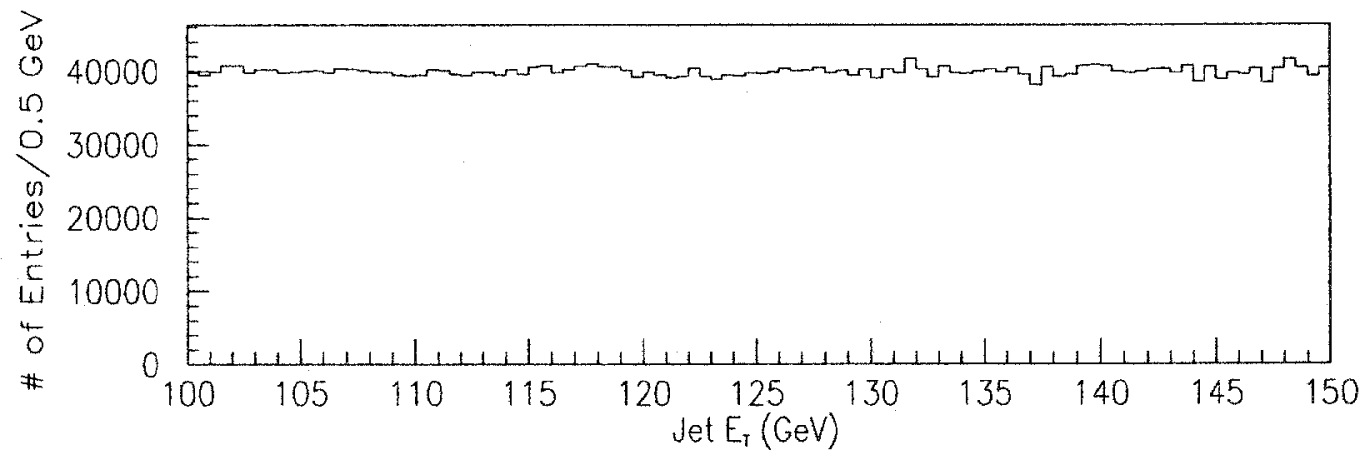

Jet $E_{T}$ (Weighted by Flat Distribution)

Figure 8.4: The weighted jet $E_{T}$ for $20-50 \mathrm{GeV}$ (top), $50-100 \mathrm{GeV}$ (middle), and $100-150 \mathrm{GeV}$ (bottom) jets. For all jet $E_{T}$ ranges, the jet $E_{T}$ spectrum has been weighted to yield the flat distribution vs. jet $E_{T}$. Both the number of tracks per jet and the jet $E_{T}$ trigger effects have been taken into account. 


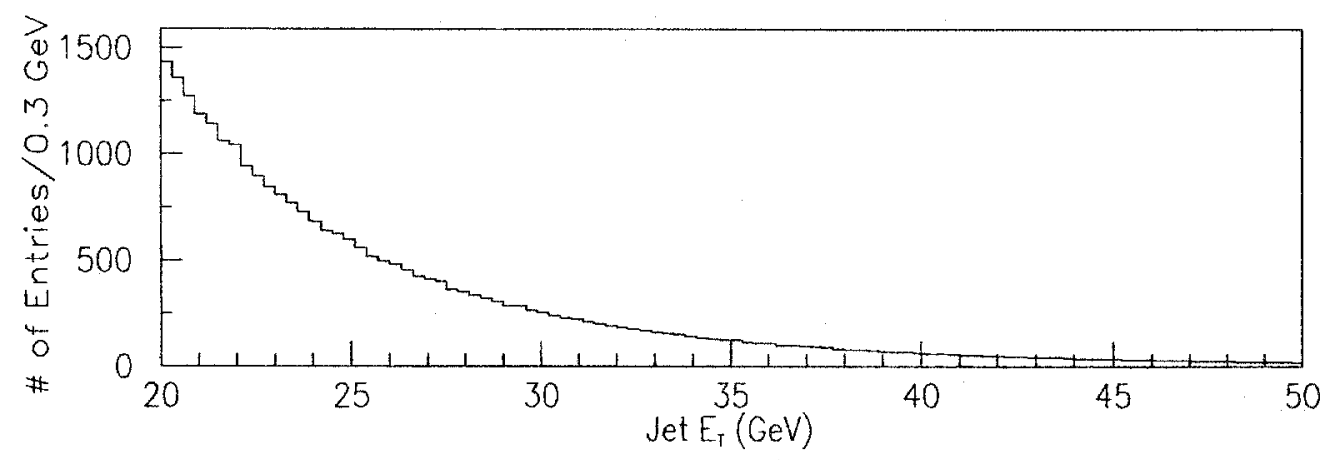

Jet $E_{T}$ (Weighted by Shape of Jet Cross-Section Distribution)

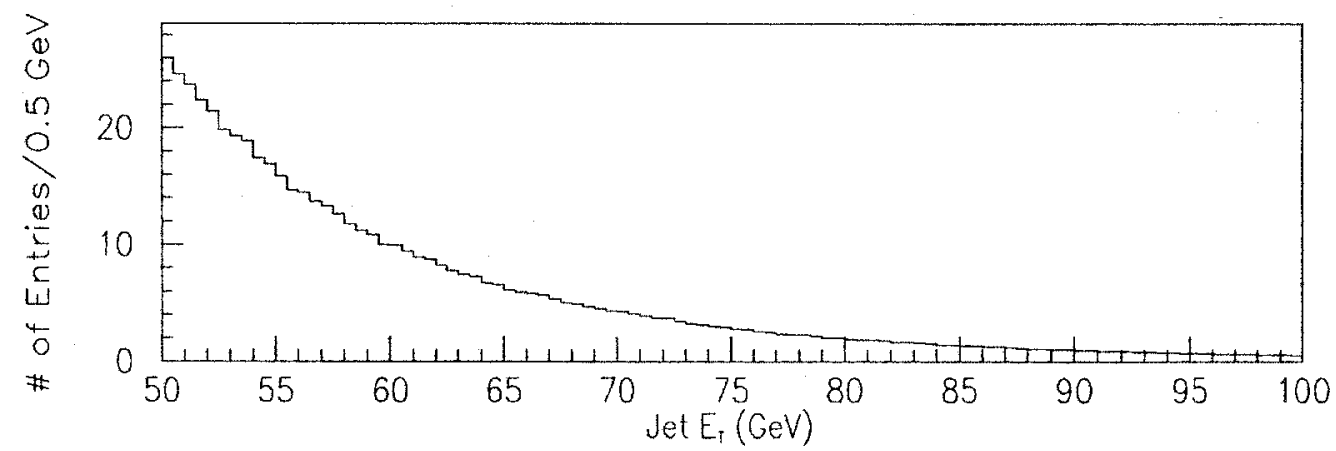

Jet $E_{\mathrm{T}}$ (Weighted by Shope of Jet Cross-Section Distribution)

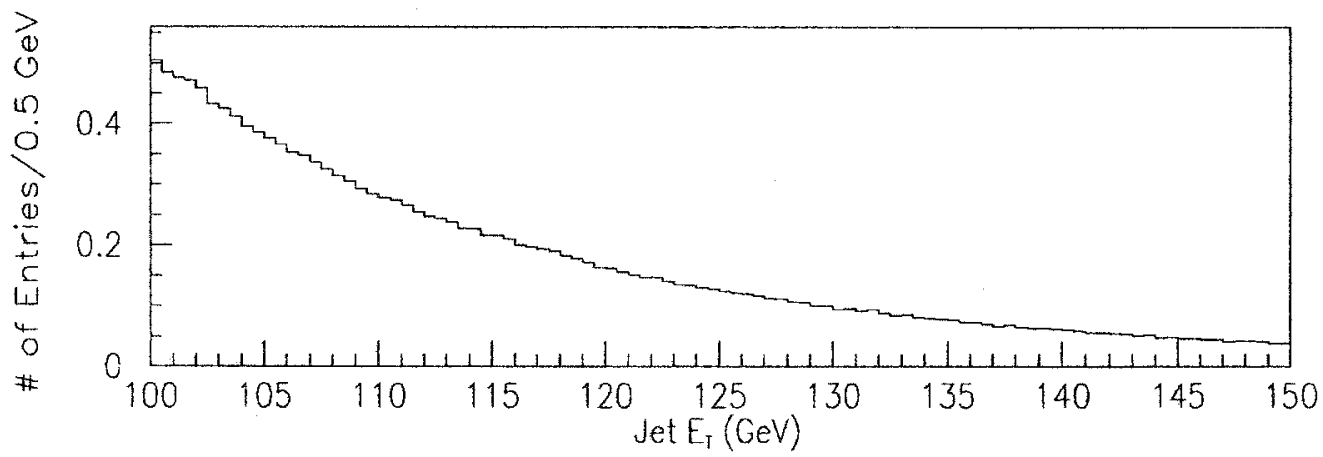

Jet $E_{T}$ (Weighted by Shape of Jet Cross--Section Distribution)

Figure 8.5: The weighted jet $E_{T}$ for $20-50 \mathrm{GeV}$ (top), $50-100 \mathrm{GeV}$ (middle), and $100-150 \mathrm{GeV}$ (bottom) jets. For all jet $E_{T}$ ranges, the jet $E_{T}$ spectrum has been weighted to yield the same distribution as the jet cross-section vs. jet $E_{T}$. Both the number of tracks per jet and the jet $E_{T}$ trigger effects have been taken into account. 


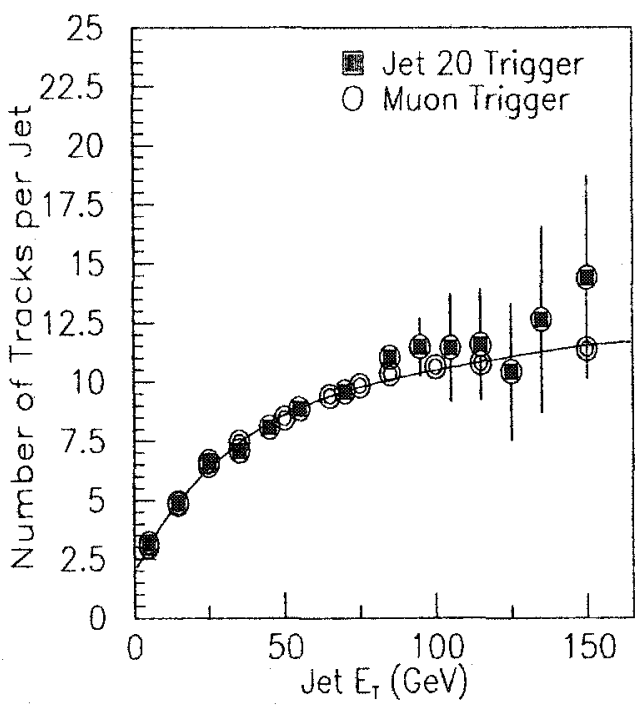

Tracks per Jet vs. Jet $E_{\mathrm{T}}$



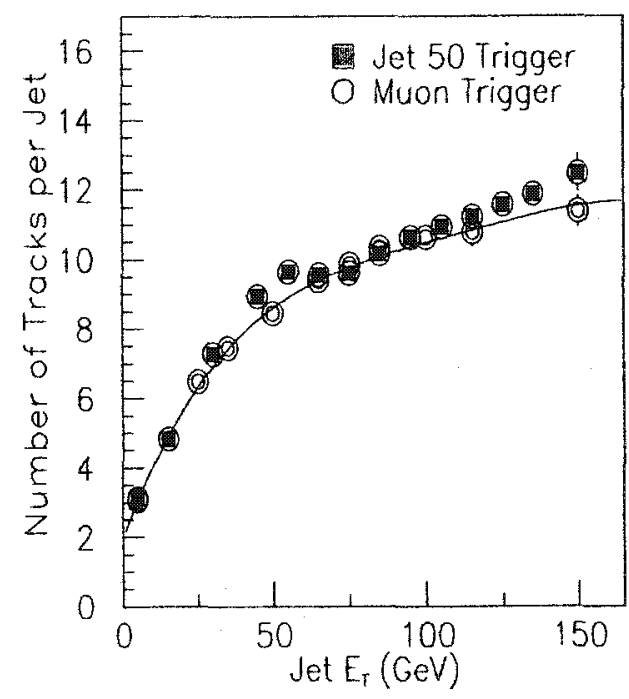

Trocks per Jet vs. Jet $E_{T}$

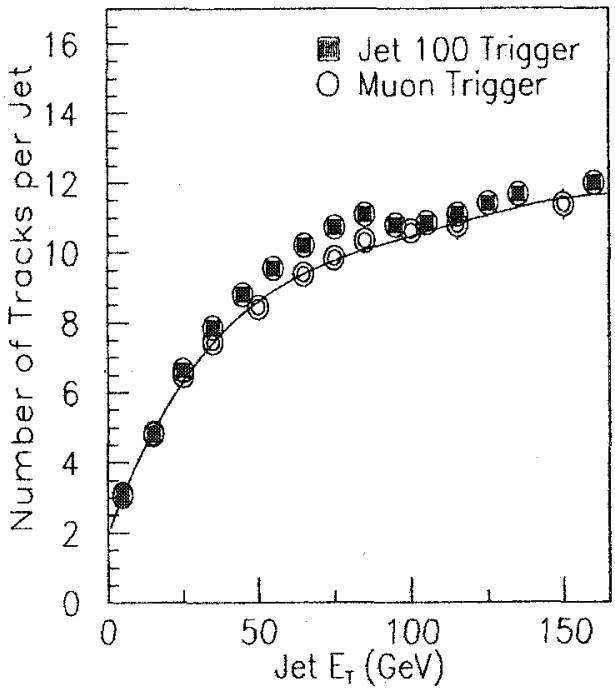

Tracks per Jet vs. Jet $E_{T}$

Figure 8.6: The jet trigger dependence on the number of tracks per jet vs. jet $E_{\mathrm{T}}$. In top left, the number of tracks per jet vs. jet $E_{T}$ dependence is plotted for both the JET20 and the muon sets. The squares are the JET20 set, and the circles are for the muon set. The muon set is fitted with a smooth curve. Similar, plots for JET50 (top right), JET70 (bottom left), and JET100 (bottom right) data sets are shown. 


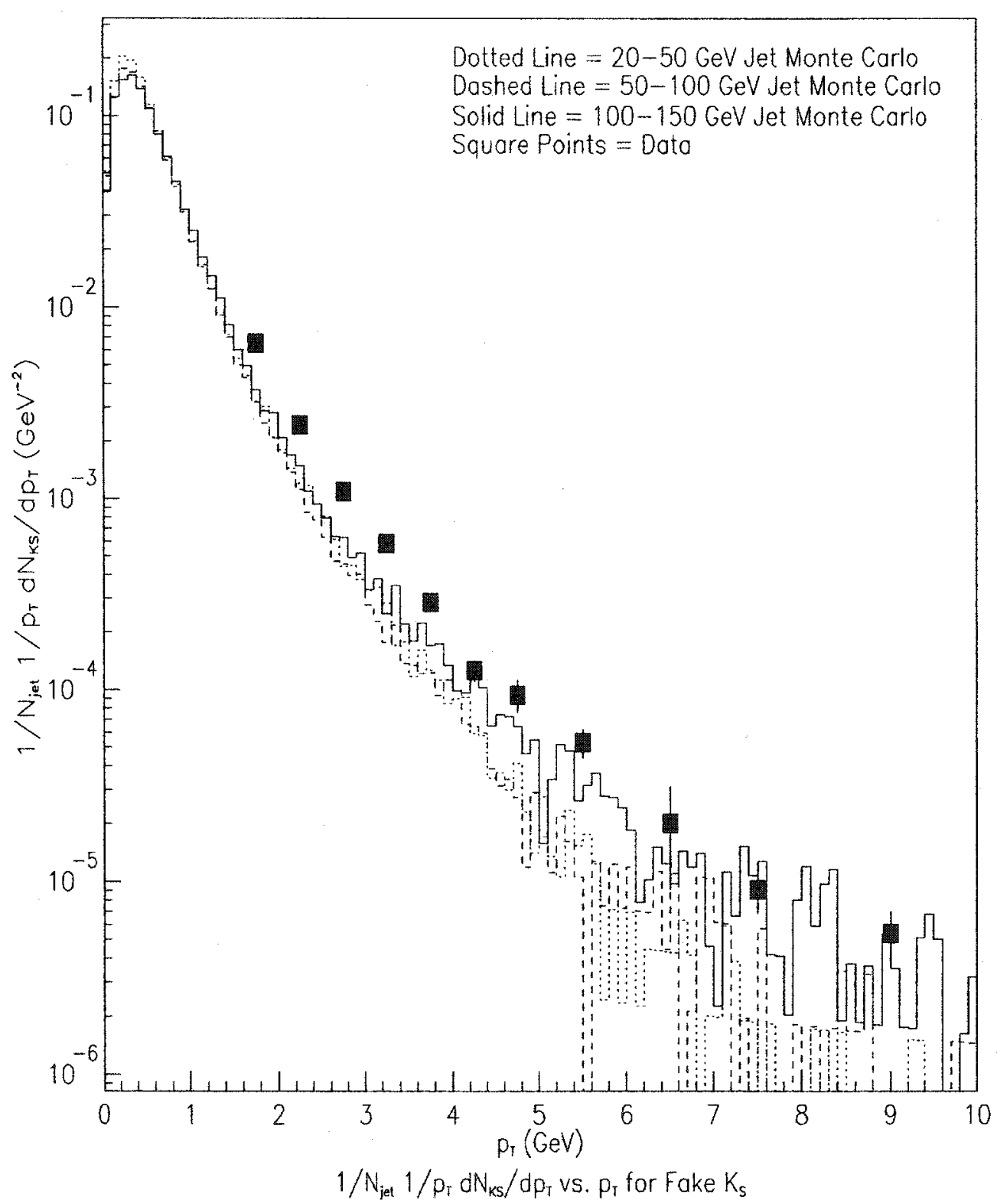

Figure 8.7: The $\frac{1}{N_{\text {jet }}} \frac{1}{p_{T}} \frac{d N_{K S}}{d p_{T}}$ distribution for background $K_{S}$ in jets for data and HERWIG Monte Carlo. This is described further in Chapter 9. The statistical errors on the HERWIG Monte Carlo histograms are on the order of 1.2E-04, 1.2E04 , and $1.5 \mathrm{E}-04$ at $2.0 \mathrm{GeV}$ for $20-50 \mathrm{GeV}, 50-100 \mathrm{GeV}$, and $100-150 \mathrm{GeV}$ jets, $6.3 \mathrm{E}-06,9.2 \mathrm{E}-06$, and $1.3 \mathrm{E}-05$ at $5.0 \mathrm{GeV}$ for $20-50 \mathrm{GeV}, 50-100 \mathrm{GeV}$, and $100-150$ $\mathrm{GeV}$ jets, and 2.0E-06, $4.7 \mathrm{E}-06$, and $3.3 \mathrm{E}-06$ at $7.0 \mathrm{GeV}$ for $20-50 \mathrm{GeV}, 50-100 \mathrm{GeV}$, and $100-150 \mathrm{GeV}$ jets. 


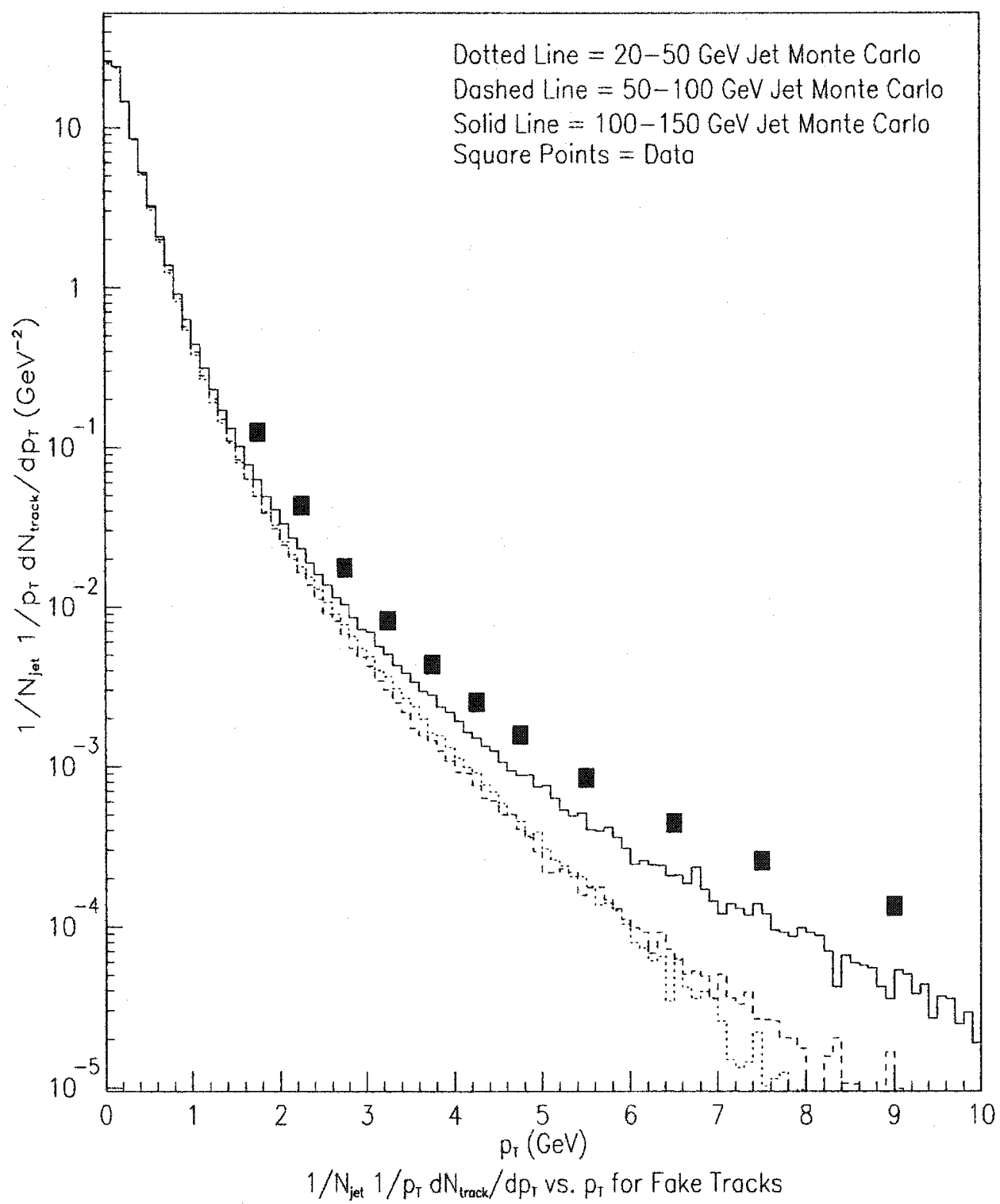

Figure 8.8: The $\frac{1}{N_{j e t}} \frac{1}{p_{T}} \frac{d N_{\text {track }}}{d p_{T}}$ distribution for background tracks in jets for data and HERWIG Monte Carlo. This is described further in Chapter 9. The statistical errors on the HERWIG Monte Carlo histograms are on the order of 4.0E-04, 3.9E-04 and $4.7 \mathrm{E}-04$ at $2.0 \mathrm{GeV}$ for $20-50 \mathrm{GeV}, 50-100 \mathrm{GeV}$, and $100-150 \mathrm{GeV}$ jets, $2.7 \mathrm{E}-05$, $2.4 \mathrm{E}-05$, and $4.1 \mathrm{E}-05$ at $5.0 \mathrm{GeV}$ for $20-50 \mathrm{GeV}, 50-100 \mathrm{GeV}$, and $100-150 \mathrm{GeV}$ jets, and 7.1E-06, 7.1E-06, and 1.5E-05 at 7.0 GeV for $20-50 \mathrm{GeV}, 50-100 \mathrm{GeV}$, and $100-150 \mathrm{GeV}$ jets. 


\section{Chapter 9}

\section{Jets: HERWIG Monte Carlo}

The comparison of the results from the data to those calculated via HERWIG Monte Carlo are an important part of this analysis. To initiate this discourse, the exact procedure of extracting the HERWIG Monte Carlo $K_{S}$ and tracks contained within the jets will be described. The HERWIG Monte Carlo requires some adjustments reminiscent of those applied to the data in Chapter 8. For example, the setting of certain Monte Carlo parameters skews the jet $E_{T}$ spectra in a manner which mirrors that of the data triggers. Furthermore, the HERWIG Monte Carlo background $K_{S}$ and tracks from non-fragmentation processes also make their way inside the jets. Both of these effects need to be examined for prior to arriving at any conclusions about the track and $K_{S}$ production inside jets. After these effects are accounted for in the HERWIG Monte Carlo, the stage is set to contrasting the data with the Monte Carlo.

\subsection{HERWIG Monte Carlo}

This would be an excellent time to briefly describe the Monte Carlo results employed in the comparison to the data. What is of interest is the various distributions of the $K_{S}$ and tracks inside the jets, and to achieve this goal, the information of the 
particles and the jets are extracted at different stages of the HERWIG generator and the QFL simulator procedure.

The particles of the jets are produced using the $2 \rightarrow 2$ parton hard-scattering processes in the HERWIG generator. For every proton and antiproton collision, a single parton from the proton will interact with another parton from the antiproton, and the two incoming partons will undergo $2 \rightarrow 2$ hard-scattering and leave just the two outgoing partons. These outgoing partons can fragment into groups of particles later clustered into jets. The hard-scattering momenta of the colliding partons are given by the default parton distribution functions. Besides this, the range of the transverse momenta of the interacting partons may be set by tuning the parameters which change the transverse momenta of the particles as well as the $E_{T}$ of jets comprising of groups of particles [31].

The 4-vector information of the particles (the $K_{S}$ and the tracks) is obtained directly after the HERWIG generator. At this level, the $K_{S}$ are identified by their particle IDs. Equivalently, the tracks are considered as the particles having the particle IDs of charged pions $\left(\pi^{+}\right.$and $\left.\pi^{-}\right)$, charged kaons $\left(K^{+}\right.$and $\left.K^{-}\right)$, electrons $\left(e^{-}\right)$, positrons $\left(e^{+}\right)$, muons $\left(\mu^{-}\right)$, antimuons $\left(\mu^{+}\right)$, protons $(p)$, and antiprotons $(\bar{p})$. At this point, the $p_{T}$ corrected distributions of the $K_{S}$ and the tracks are determined. Next, the $K_{S}$ and the tracks contained within jets need to be identified.

A jet is an entity that depends upon the clustering algorithm applied to the energy clusters in the calorimeter. Clearly, at the HERWIG generator level, this calorimetry information has not yet been created. Hence, the HERWIG generator level is used as the input for the QFL simulator [90]. QFL is a simulator (CDFSIM described Chapter 7 is another) which does tracking poorly and calorimetry reasonably well. After the QFL simulator, the detector information produced is ran 
through the same clustering algorithms and the jet energy corrections as the data in order to identify jets as well as compute the jet quantities like the jet vertices and the jet $E_{T}$.

If either a HERWIG level $K_{S}$ or track is found within a $\Delta R=0.7$ cone of a HERWIG+QFL+Cluster Code jet, then the particle is considered to be inside this jet. In particular, the following cuts are performed on the jets, the tracks, and the $K_{S}$ :

- Jet Cuts

$$
\begin{aligned}
& -\left|z_{\text {primary }}\right|<60.0 \mathrm{~cm} \\
& -z_{\text {jet }}^{\text {rms }}<5.0 \mathrm{~cm} \\
& -\left|z_{\text {primary }}-z_{\text {jet }}\right|<5.0 \mathrm{~cm} \\
& -\left|\eta^{\text {jet }}\right|<1.0 \\
& -20.0<E_{T}{ }^{j e t}<50.0 \mathrm{GeV}, 50.0<E_{T}^{j e t}<100.0 \mathrm{GeV} \text {, or } 100.0<E_{T}^{j e t}<150.0 \\
& \quad \mathrm{GeV}
\end{aligned}
$$

- Track Cuts

$$
\begin{aligned}
& -\left|z_{\text {primary }}\right|<60.0 \mathrm{~cm} \\
& -z_{j e t}^{\text {rms }}<5.0 \mathrm{~cm} \\
& -\left|z_{\text {primary }}-z_{\text {jet }}\right|<5.0 \mathrm{~cm} \\
& -\left|\eta^{j e t}\right|<1.0 \\
& -\left|\eta^{t r a c k}\right|<1.0 \\
& - \text { Pass Particle ID Criteria }\left(\pi^{+}, \pi^{-}, K^{+}, K^{-}, e^{-}, e^{+}, \mu^{-}, \mu^{+}, p, \text { and } \bar{p}\right) \\
& \text { - Tracks within } \Delta R=0.7 \text { Jet }
\end{aligned}
$$


$-20.0<E_{T}{ }^{j e t}<50.0 \mathrm{GeV}, 50.0<E_{T}{ }^{j e t}<100.0 \mathrm{GeV}$, or $100.0<E_{T}{ }^{j e t}<150.0$ $\mathrm{GeV}$

- $K_{S}$ Cuts

$-\left|z_{\text {primary }}\right|<60.0 \mathrm{~cm}$

$-z_{j e t}^{r m s}<5.0 \mathrm{~cm}$

$-\left|z_{\text {primary }}-z_{j e t}\right|<5.0 \mathrm{~cm}$

$-\left|\eta^{j e t}\right|<1.0$

$-\left|\eta^{\text {track }}\right|<1.0$

- Pass Particle ID Criteria $\left(K_{S}\right)$

- Tracks within $\Delta R=0.7$ Jet

$-20.0<E_{T}^{j e t}<50.0 \mathrm{GeV}, 50.0<E_{T}^{j e t}<100.0 \mathrm{GeV}$, or $100.0<E_{T}{ }^{j e t}<150.0$ $\mathrm{GeV}$

Since the QFL simulator reproduces tracking information poorly with $100 \%$ wire efficiency (most wires have efficiencies between 75-95\%), the value of the Monte Carlo jet vertices computed from this tracking information may be called into question. However, the jets with $E_{T}$ more than $20 \mathrm{GeV}$ usually have at least 7 tracks with momenta above $0.5 \mathrm{GeV}$, and since the tracking efficiency is greater than $90 \%$, a loss of a track or two would not radically alter the measurement. In addition, there are also tracks below $0.5 \mathrm{GeV}$ incorporated into a given jet vertex calculation.

The Monte Carlo jet properties have been studied closely by others as well as in the course of this analysis. Both types of jets have been found to be consistent. In the next two sections, the corrections to the Monte Carlo jets, the tracks in jets, and the $K_{S}$ in jets will be described. 


\section{2 "Trigger Corrections" to the Monte Carlo}

In order to compare the Monte Carlo with the data, many Monte Carlo jets at various energies need to be generated. With default values, it would be inefficient to acquire a large number of jets at a particular $E_{T}$. The greater the $E_{T}$, the more impractical the rational for the implementation of the default parameters. However, by changing the allowable range of the transverse momenta of the interacting partons in the Monte Carlo, numerous jets at any desired energy can be obtained in a reasonable time.

By adjusting the upper and lower bounds of the transverse momenta of the hard-scattering partons, a Gaussian-like distribution of jet $E_{T}$ about a jet $E_{T}$ is produced. The exact values of these parameters are set so the falling edge of this Gaussian-like jet $E_{T}$ distribution is contained within the jet $E_{T}$ region of interest. In particular, for the production of $20-50 \mathrm{GeV}, 50-100 \mathrm{GeV}$, and $100-150 \mathrm{GeV}$ jets, the limits of the transverse momenta of the partons are fixed at 10.0 and $50.0 \mathrm{GeV}$, 40.0 and $100.0 \mathrm{GeV}$, and 80.0 to $150.0 \mathrm{GeV}$, respectively.

The setting of the hard-scattering transverse momenta parameters to mold the Monte Carlo jet $E_{T}$ is analogous to how the jet triggers bias the jet $E_{T}$ of the data. Likewise, the Monte Carlo jet $E_{T}$ distributions are corrected similarly by weighting each jet $E_{T}$ value so the jet $E_{T}$ plots show a flat constant of 40,000 (any constant would do). Then, the jet $E_{T}$ spectrum for each of the $E_{T}$ ranges is weighted in order for the resulting histogram to be similar in shape to the jet cross-section as a function of jet $E_{T}$. The $K_{S}$ and tracks inside jets are weighted according to the $E_{T}$ of the jet in which they are embedded. As for the number of tracks per jet trigger correction for the Monte Carlo, there is none, since the setting of the hard-scattering transverse momenta range parameters do not effect the number of 
tracks per jet.

\subsection{Background Corrections to the Monte Carlo}

The background $K_{S}$ and tracks in jets are also removed from the $K_{S}$ and tracks in jets in the HERWIG Monte Carlo. These fake rates for the $K_{S}$ and tracks are expected to differ from the background rates of the data since multiple interactions, quite common in data, do not occur with the default Monte Carlo settings. However, like the case for the data, the background effect should not be more than $10 \%$ and most of the contribution should be confined to the first few low $p_{T}$ bins.

The techniques incorporated to calculate the background $K_{S}$ and tracks contained within jets in the Monte Carlo closely overlap those previously used for the data. An arbitrary cone with $\left|\eta_{j e t}^{\text {fake }}\right|<1.0$ and $0 \leq \phi_{j e t}^{\text {fake }}<2 \pi$ is first isolated from all jets above $10 \mathrm{GeV}$, then the number of $K_{S}$ and tracks inside the jets is tallied after making use of the particle ID and the 4-vector information taken directly from the HERWIG Monte Carlo. The isolation criteria depends on the jets above $10 \mathrm{GeV}$ obtained after the HERWIG Monte Carlo and the QFL simulator stages. For the fake rates of the $K_{S}$ and tracks in HERWIG Monte Carlo jets, the following cuts are employed for the jets, the tracks, and the $K_{S}$ :

- Fake Jet Cuts

$-\left|z_{\text {primary }}\right|<60.0 \mathrm{~cm}$

- Select $\eta_{\text {jet }}^{\text {fake }}$ with $\left|\eta_{\text {jet }}^{\text {fake }}\right|<1.0$

- Select $\phi_{\text {jet }}^{\text {fake with } 0.0 \leq \phi_{\text {jet }}^{\text {fake }}<2 \pi}$

- For all jets with $E_{T}>10.0 \mathrm{GeV},\left|\Delta R_{\text {jet-fake jet }}\right|>1.0$ 
- Track Cuts

$-\left|z_{\text {primary }}\right|<60.0 \mathrm{~cm}$

- Pass Particle ID Criteria $\left(\pi^{+}, \pi^{-}, K^{+}, K^{-}, e^{-}, e^{+}, \mu^{-}, \mu^{+}, p\right.$, and $\left.\bar{p}\right)$

$-\Delta R_{\text {track-fake jet }}<0.7$

$-\left|\eta^{\text {track }}\right|<1.0$

$-1.5<p_{T}^{\text {track }}<10.0 \mathrm{GeV}$

- $K_{S}$ Cuts

$-\left|z_{\text {primary }}\right|<60.0 \mathrm{~cm}$

- Pass Particle ID Criteria $\left(K_{S}\right)$

$-\Delta R_{\text {track-fake }} j e t<0.7$

$-\left|\eta^{K S}\right|<1.0$

$-1.5<p_{T}^{K S}<10.0 \mathrm{GeV}$

On the other hand, since the hard-scattering variables are set separately for the 20-50 GeV, $50-100 \mathrm{GeV}$, and $100-150 \mathrm{GeV}$ jets, there are actually three separate curves for the background $K_{S}$ and tracks contained within jets. Furthermore, each separate HERWIG Monte Carlo background plot is subtracted bin by bin from the appropriate corresponding histogram of either the number of $K_{S}$ per jet or the number of tracks per jet for a given jet $E_{T}$ range. The number of HERWIG Monte Carlo background $K_{S}$ per jet is $0.0073 \pm 0.0001,0.0059 \pm 0.0001,0.007 \pm 0.0001$ for the $20-50 \mathrm{GeV}, 50-100 \mathrm{GeV}$, and $100-150 \mathrm{GeV}$ cases, and the number of HERWIG Monte Carlo background track per jet is $0.1055 \pm 0.0004,0.0873 \pm 0.0003$, and $0.1081 \pm 0.0003$ for the $20-50 \mathrm{GeV}, 50-100 \mathrm{GeV}$, and $100-150 \mathrm{GeV}$ cases. The 
background rates for the number of tracks and the number of $K_{S}$ inside jets are at most a $5 \%$ effect.

\subsection{Comparing the Background of the Data and the HERWIG Monte Carlo}

In order to produce Figure 8.7 and 8.8 , the $p_{T}$ distributions of HERWIG Monte Carlo background $K_{S}$ and tracks inside jets are divided by the number of jets, the bin size, and the $p_{T}$ value at the midpoint of the bin. These $\frac{1}{N_{j e t}} \frac{1}{p_{T}} \frac{d N_{K S}}{d p_{T}}$ and $\frac{1}{N_{j e t}} \frac{1}{p_{T}} \frac{d N_{\text {track }}}{d p_{T}}$ are shown respectively for the $20-50,50-100$, and $100-150 \mathrm{GeV}$ jet $E_{T}$ ranges along with the equivalent background rates from the data. The data fake rate is greater than the Monte Carlo fake rates. Whereas in the data, the number of $K_{S}$ and tracks per jet for $1.5 \mathrm{GeV}<p_{T}<10.0 \mathrm{GeV}$ are $0.0125 \pm 0.0003$ and $0.2237 \pm 0.0003$, respectively, the equivalent quantities from the HERWIG+QFL Monte Carlo are only around 0.005 to 0.007 for the background $K_{S}$ per jet and .1 for the background tracks per jet. The data is approximately a factor of 2.0 higher than the HERWIG+QFL Monte Carlo. One reason for this is believed to be due to multiple interactions. There may be more than one interaction in a given data event whereas every default Monte Carlo event has only a single interaction. Thus, it is possible for the data to have either a $K_{S}$ or a track originating from a different interaction point near the primary vertex be counted along with the other particles that do come from the primary.

Another reason for the discrepancy may originate from the limitations in characterizing the "fake" jets. Unlike the "real" jets, "fake" jets have neither a jet vertex nor a jet $E_{T}$ associated with them. Moreover, correcting for the efficiencies 
with the curve for the track-embedded $K_{S}$ (and tracks) inside 20-50 GeV jets may be inappropriate. So if this efficiency is underestimated, then the correction factor might be larger than it should. Consequently, the background in the data would be computed to be too high.

Aside from the overall normalization factor, the shape of the background in the data closely resembles the $100-150 \mathrm{GeV}$ Monte Carlo. This is believed to be so because the data is dominated by jets with $E_{T}$ between $100-150 \mathrm{GeV}$. As for the 20-50 GeV and 50-100 GeV Monte Carlo cases having their distributions extending not as far in $p_{T}$ as the $100-150 \mathrm{GeV}$ Monte Carlo case, this is attributed to the likely possibility that the jets with greater $E_{T}$ have a higher $p_{T}$ background.

\subsection{Comparing Background with Other Analyses}

There are a couple of studies that should be mentioned, and both results have significantly higher track background rates than those stated here, referred to as "study 0", for both the data and HERWIG Monte Carlo.

In the first analysis, denoted by "study 1", [28] [82] [83], the background rate of tracks in dijets is computed by using a pair of "fake" 0.47 cones having the same jet $\eta$ direction of the leading jet in a given dijet event but having the $\phi$ component of the "fake" jet rotated by $90^{\circ}$ from that of the jet $\phi$ of the leading jet. Both "fake" 0.47 cones are collected statistically to yield a background rate of approximately 0.5-0.6 tracks per jet in the data.

In another analysis referred to as "study 2" ( [84] [85] [86]), the background track rate in "fake" 0.7 cones is reported for all jet events. Likewise, a pair of "fake" jets are $90^{\circ}$ in $\phi$ from the leading jet direction while having the same jet $\eta$ as the leading jet. However, only the "fake" jet with the lowest $E_{T}$ deposition in the calorimeter 
of the two "fake" jets is considered in the track background rate computation. This track background rate is also calculated to be around 0.5 tracks per jet in the data, and this quantity appears to be constant for all jet $E_{T}$.

Both studies 1 and 2 have track background rates (for the data) larger than the $0.2237 \pm 0.0003$ tracks per jet value stated in study 0 (this analysis). However, these other analyses (study 1 and 2) use different $p_{T}$ ranges to compute their values. In study 1 ( [84] [85] [86]), the $p_{T}$ range is from $0.4 \mathrm{GeV}$ and above, and in study 2 ( [28] [82] [83]), the $p_{T}$ range is from $0.5 \mathrm{GeV}$ and above. This analysis, study 0 , implements a much higher $p_{T}$ range, from $1.5 \mathrm{GeV}$ to $10.0 \mathrm{GeV}$. Virturally all of this difference is expected to be between $0.4 \mathrm{GeV}$ (or $0.5 \mathrm{GeV}$ ) and $1.5 \mathrm{GeV}$ since the number of tracks above $10.0 \mathrm{GeV}$ is small.

If study 0 implements similar ranges, these results may be contrasted with studies 1 and 2. For $0.4 \mathrm{GeV}(0.5 \mathrm{GeV})$ to $10.0 \mathrm{GeV}$, this analysis, study 0 , gets about $1.1(0.95)$ tracks per jet for the data. This extrapolation is carried out into a region where there are no data points, and the uncertainty is large. In addition, multiple interactions included in the data that would increase this rate above the 0.5-0.6 tracks per jet values in study 2 ( [28] [82] [83]), and the 0.5 tracks per jet quantity quoted in study 1 ( [84] [85] [86]). In any case, even a factor of 2.0 difference in the background rate would only effect the first few $p_{T}$ bins by at most $5 \%$. There is not another analysis that examines the background $K_{S}$ rate to make a similar comparison.

Despite the data rates, the HERWIG+Monte Carlo background rates are equivalent. From the other publications described in Chapter 8, the tracks in dijets in study 1 ( [73], [74], and [75]) and the tracks in jets in study 2 ( [76], [82], and [83]), the HERWIG+QFL track background rates are also quoted. For the former, study 
1 , the track background rate varies from 0.3 to 0.8 tracks per jet depending on the mass of the dijet, and for the latter, study 2 , the background rate is approximately 0.6 tracks per jet. Although both quantities are above the $0.175 \pm 0.002$ track per jet calculated in study 0 (this study), once the $p_{T}$ range is adjusted to be consistant, the agreement improves considerably. For $p_{T}$ between $0.4 \mathrm{GeV}$ and $10.0 \mathrm{GeV}$, study 0 gets around 0.55 tracks per jet for HERWIG+QFL. This is within the range of the 0.3-0.8 tracks per jet in study 1 ( [28] [82] [83]). In addition, for $0.5 \mathrm{GeV}$ to 10.0 GeV, this analysis yields between 0.45 to 0.49 tracks per jet. Again, this value is near the 0.6 tracks per jet quantity quoted in study 2 ([84] [85] [86]).

These differences are not expected to alter the results much since the total background correction is less than $8 \%$, and they may be attributed to the differences in computation of these background rates from analysis to analysis such as the cone size and the definition of jet. The exact details will be spared, but in study 1 ( [73], [74], and [75]), the cone-size is 0.47 and the $\eta$ range is within $|\eta|<0.7$. Furthermore, in study 2 ( [76], [82], and [83]), the jets are defined through charged tracks rather than calorimetry information. Despite this, the background rates agree with the Monte Carlo, and the differences between in the background rate in the data is believed to be due to multiple interactions. The net effect will be small.

\subsection{The HERWIG Monte Carlo Results}

The number $K_{S}$ per jet, the number of tracks per jet, and 2.0 times the number of $K_{S}$ per track are shown for the HERWIG+QFL Monte Carlo after each correction in Tables 9.1-9.3. To render greater detail, in Table 9.1, after the jet $E_{T}$ "trigger" correction, the number of $K_{S}$ per jet inside the 20-50 GeV HERWIG Monte Carlo jets is $0.175 \pm 0.002$. These $K_{S}$ are contained within jets which are distributed 
according to the shape of the jet $E_{T}$ cross-section. Being HERWIG Monte Carlo $K_{S}, p_{T}$ efficiency corrections and the number of tracks per jet trigger adjustments are unnecessary. Hence, the number remains $0.162 \pm 0.002$ in the next row. After the fake rate has been subtracted out, there are $0.155 \pm 0.002 K_{S}$ per jet in the 20-50 GeV HERWIG Monte Carlo jets. Note that the tracks per jets behave equivalently in Table 9.2, and 2.0 times the $K_{S}$ per track are virtually unchanged in Table 9.3.

In comparing the data with the HERWIG+QFL Monte Carlo, Tables 9.1-9.3 will be examined along with Tables 8.1-8.3. First, in Table 9.1, the number of $K_{S}$ in jets in for $20-50 \mathrm{GeV}, 50-100 \mathrm{GeV}$, and $100-150 \mathrm{GeV}$ cases after all corrections in the HERWIG+QFL Monte Carlo is $0.155 \pm 0.002,0.259 \pm 0.001$, and $0.313 \pm 0.001$. Note that there is definately an increase. However, in the data, the number of $K_{S}$ inside jets is $0.156 \pm 0.002,0.206 \pm 0.003$, and $0.200 \pm 0.003$. So, for the data, the $K_{S}$ production inside jets reaches a plateau despite the agreement for $20-50 \mathrm{GeV}$. This is not the case with track production inside jets; the HERWIG+QFL and the data agree within $10 \%$ for all jet $E_{T}$. In addition, from Table 9.2, the number of tracks inside jets from the HERWIG+QFL Monte Carlo increases from $2.839 \pm 0.012$ to $6.641 \pm 0.007$ from the $20-50 \mathrm{GeV}$ case to the $100-150 \mathrm{GeV}$ case. Similarly, from Table 8.2, the number of tracks inside jets from the data increases from $2.802 \pm 0.002$ to $5.957 \pm 0.003$ from the $20-50 \mathrm{GeV}$ case to the $100-150 \mathrm{GeV}$ case. As a result of the $K_{S}$ production discrepancy between the data and HERWIG+QFL increasing with jet $E_{T}$ while the track production is in agreement, the $\left(2^{*} K_{S}\right)$ per track ratios in Tables 9.3 and 8.3 diverge as well. This will be discussed further in Chapter 10.

At least two other analyses have discussed the topic of the number of tracks per jet. In study 1 ( [28] [82] [83]), the number of tracks per jet as a function of dijet mass is examined. Granted there are differences from study 0 , such as the 
lower $400 \mathrm{MeV}$ track cut among others; nonetheless, for the data, the number of tracks inside a 0.47 cone jet in study 1 increases from $6.1 \pm 0.0 \pm 0.5$ for dijets of mass $82 \mathrm{GeV} / c^{2}$ to $10.3 \pm 0.1 \pm 0.7$ for dijets of mass $293 \mathrm{GeV} / c^{2}$. Using a similar $p_{T}$ range $\left(0.4 \mathrm{GeV}<p_{T}<10.0 \mathrm{GeV}\right)$ for the data in study 0 , the number of tracks per jet is $5.019 \pm 0.013,8.134 \pm 0.013$, and $8.763 \pm 0.010$ for $20-50 \mathrm{GeV}$, 50-100 GeV, and 100-150 GeV jets, respectively. Even though dijets are different objects than jet $E_{T}$, the number of tracks per jet appear to be comparable. For HERWIG+QFL and the identical $p_{T}$ range in study 0 , the number of tracks per jet is $4.752 \pm 0.034,7.770 \pm 0.020$, and $9.715 \pm 0.015$ for $20-50 \mathrm{GeV}, 50-100 \mathrm{GeV}$, and $100-150 \mathrm{GeV}$ jets, respectively. The important to note that in study 1 , the data results are approximately $11 \%$ below those from the HERWIG Monte Carlo (not shown for study 1). This is also true for study 0 .

The other analysis, study 2 ( [87], [88], and [89]) calculates the number of tracks per jet in a manner similar to here, but the jets are computed strictly from tracks instead of calorimetry. Again, the details have no place here since there is an obvious bias of constructing jets from tracks. Now, a $20 \mathrm{GeV}$ jet computed from just tracks would correspond to a much higher $E_{T}$ jet calculated from the calorimeter. Nevertheless, there are about 6.0 tracks inside a 0.7 cone, $20 \mathrm{GeV} p_{T}$ jet, and this value increases to 10.0 tracks per jet for $50 \mathrm{GeV} p_{T}$ jets. This is not necessarily inconsistent with study 0 since the data also deviates from the HERWIG Monte Carlo as jet $E_{T}$ increases. If study 0 uses the identical $p_{T}\left(0.5 \mathrm{GeV}<p_{T}<10.0 \mathrm{GeV}\right)$ range, the number of tracks per jet is $4.809 \pm 0.012,7.837 \pm 0.012$, and $8.505 \pm 0.010$ for $20-50 \mathrm{GeV}, 50-100 \mathrm{GeV}$, and $100-150 \mathrm{GeV}$ jets, respectively. Similiarly, for the HERWIG+QFL results in study 0 , the number of tracks per jet is $4.575 \pm 0.033$, $7.508 \pm 0.020$, and $9.410 \pm 0.014$ for $20-50 \mathrm{GeV}, 50-100 \mathrm{GeV}$, and $100-150 \mathrm{GeV}$ jets, 
Table 9.1: The number of $K_{S}$ per Jet in the HERWIG Monte Carlo $\left(1.5<p_{T}<10.0 \mathrm{GeV}\right)$.

\begin{tabular}{|c|c|c|c|}
\hline $\begin{array}{c}\text { After } \\
\text { Correction }\end{array}$ & $\begin{array}{c}\frac{N_{K S}}{j e t} \\
\text { in 20-50 Jets }\end{array}$ & $\begin{array}{c}\frac{N_{K S}}{j e t} \\
\text { in 50-100 Jets }\end{array}$ & $\begin{array}{c}\frac{N_{K S}}{j e t} \\
\text { in } 100-150 \text { Jets }\end{array}$ \\
\hline$P_{T}$ & $0.162 \pm 0.002$ & $0.265 \pm 0.001$ & $0.319 \pm 0.001$ \\
Trigger & $0.162 \pm 0.002$ & $0.265 \pm 0.001$ & $0.319 \pm 0.001$ \\
Fake Rate & $0.155 \pm 0.002$ & $0.259 \pm 0.001$ & $0.313 \pm 0.001$ \\
\hline
\end{tabular}

Table 9.2: The number of tracks per jet in HERWIG Monte Carlo $\left(1.5<p_{T}<10.0 \mathrm{GeV}\right)$.

\begin{tabular}{|c|c|c|c|}
\hline $\begin{array}{c}\text { After } \\
\text { Correction }\end{array}$ & $\begin{array}{c}\frac{N_{\text {track }}}{\text { jet }} \\
\text { in 20-50 Jets }\end{array}$ & $\begin{array}{c}\frac{N_{\text {track }}}{\text { jet }} \\
\text { in 50-100 Jets }\end{array}$ & $\begin{array}{c}\frac{N_{\text {track }}}{\text { jet }} \\
\text { in 100-150 Jets }\end{array}$ \\
\hline$P_{T}$ & $2.945 \pm 0.012$ & $5.170 \pm 0.006$ & $6.749 \pm 0.007$ \\
Trigger & $2.945 \pm 0.012$ & $5.170 \pm 0.006$ & $6.749 \pm 0.007$ \\
Fake Rate & $2.839 \pm 0.012$ & $5.083 \pm 0.006$ & $6.641 \pm 0.007$ \\
\hline
\end{tabular}

Table 9.3: 2.0 times the number of $K_{S}$ per track in HERWIG Monte Carlo $\left(1.5<p_{T}<10.0 \mathrm{GeV}\right)$.

\begin{tabular}{|c|c|c|c|}
\hline $\begin{array}{c}\text { After } \\
\text { Correction }\end{array}$ & $\begin{array}{c}2 \frac{N_{K S}}{N_{\text {track }}} \\
\text { in } 20-50 \text { Jets }\end{array}$ & $\begin{array}{c}2 \frac{N_{K S}}{N_{\text {track }}} \\
\text { in } 50-100 \text { Jets }\end{array}$ & $\begin{array}{c}2 \frac{N_{K S}}{N_{\text {track }}} \\
\text { in } 100-150 \text { Jets }\end{array}$ \\
\hline$P_{T}$ & $0.110 \pm 0.001$ & $0.102 \pm 0.0003$ & $0.095 \pm 0.0003$ \\
Trigger & $0.110 \pm 0.001$ & $0.102 \pm 0.0003$ & $0.095 \pm 0.0003$ \\
Fake Rate & $0.109 \pm 0.001$ & $0.102 \pm 0.0003$ & $0.094 \pm 0.0003$ \\
\hline
\end{tabular}

respectively. Again, the agreement can be seen.

A detailed comparison between the data and HERWIG+QFL for the tracks and $K_{S}$ production inside jets will occur later in Chapter 10 along with the conclusion. 


\section{Chapter 10}

\section{Jets: Results and Conclusions}

The production of $K_{S}$ and tracks inside jets will now be elaborated in greater detail. After summarizing the implementation of the $p_{T}$, trigger, and background corrections to the $p_{T}$ curves, the $\frac{1}{N_{j e t}} \frac{1}{p_{T}} \frac{d N_{K S}}{d p_{T}}$ and $\frac{1}{N_{j e t}} \frac{1}{p_{T}} \frac{d N_{t r a c k}}{d p_{T}}$ spectra from the data and the HERWIG+QFL Monte Carlo are compared. Then, the number of $K_{S}$ per jet $\left(\frac{N_{K S}}{j e t}\right)$, the number of tracks per jet $\left(\frac{N_{\text {track }}}{\text { jet }}\right)$, and the $\left\langle p_{T}>\right.$ for both $K_{S}$ and tracks in jets are derived from the fit parameters of these spectra. Following this, the fragmentation plots of the $K_{S}$ in jets are contrasted with $e^{+} e^{-}$results from other experiments. Finally, bringing this analysis to a close, the significance of the measurement in terms of the fragmentation model will be discussed.

\subsection{Correcting for Tracks and $K_{S}$ Inside Jets}

As mentioned earlier in Chapter 8, to generate the $\frac{1}{N_{j e t}} \frac{1}{p_{T}} \frac{d N_{\text {track }}}{d p_{T}}$ and $\frac{1}{N_{j e t}} \frac{1}{p_{T}}$ $\frac{d N_{K S}}{d p_{T}}$ spectra requires many steps and corrections. Once the $K_{S}$ candidates and tracks in jets meet the selection criteria, the number of candidates with a given mass are grouped according to intervals of $p_{T}$. Moreover, prior to subtracting the background, these candidates are weighted by the $p_{T}$ efficiency corrections and the trigger adjustments simultaneously. By doing this, the resulting background- 
subtracted curve is already corrected for the $p_{T}$ and the trigger biases. Note that for the $p_{T}$ curve, the jet $E_{T}$ is weighted to have the same shape as the jet cross-section as a function of jet $E_{T}$ [73] [75] [76]. The fragmentation quantities, grouped into intervals of $\frac{p_{T}}{J_{e t E_{T}}}$, are also weighted so that the jet $E_{T}$ has a flat distribution. Thus, by the application of three weights for every candidate (one for the $p_{T}$ efficiency correction, one for the jet $E_{T}$ trigger adjustment, and one for the number of tracks trigger effect), the number of $K_{S}$ within a range of $p_{T}$ creates a $p_{T}$ curve needing only to account for the fake rate. As mentioned previously in Chapter 8 , through bin-by-bin subtraction of the fake rate $p_{T}$ curve from the $p_{T}$ curve, the removal of the background contributions may be accomplished.

In summary, the cuts and the corrections for the tracks and $K_{S}$ in $20-50 \mathrm{GeV}$, 50-100 GeV, and $100-150 \mathrm{GeV}$ jets are listed below:

- Track Cuts

$$
\begin{aligned}
& -\left|z_{\text {primary }}\right|<60.0 \mathrm{~cm} \\
& -z_{j e t}^{\text {rms }}<5.0 \\
& -\left|z_{\text {primary }}-z_{\text {jet }}\right|<5.0 \mathrm{~cm} \\
& -\left|\eta^{\text {jet }}\right|<1.0
\end{aligned}
$$

- Tracks are inside the nearest $\Delta R=0.7$ jet.

$-\left|z_{\text {primary }}-z_{\text {track }}\right|<5.0 \mathrm{~cm}$

$-\left|\eta^{\text {track }}\right|<1.0$

$-1.5<p_{T}^{\text {track }}<10.0 \mathrm{GeV}$

- Weight with the $p_{T}^{\text {track }}$ Efficiency Correction

- Weight with the Number of Tracks per Jet Trigger Correction 
- Weight with the Jet $E_{T}$ Trigger Correction

- Group into bins according to the range of the $p_{T}^{\text {track }}$

- $K_{S}$ Cuts

- $K_{S}$ candidates within $\Delta R=0.7$ jet cone

$-z_{j e t}^{r m s}<5.0$

$-\left|z_{\text {primary }}-z_{\text {jet }}\right|<5.0 \mathrm{~cm}$

$-\chi_{K S}^{2}<20.0$

$-\left|z_{K S^{-}} z_{\text {primary }}\right|<3.0 \mathrm{~cm}$

$-p_{T}^{\text {tracks from } K S}>500 \mathrm{MeV}$

$-\left|\eta^{K S}\right|<1.0$

- 3D Displacement ${ }^{K S}>1.0 \mathrm{~cm}$

$-\cos \theta_{P D}{ }^{K S}>0.990$

$-1.5<p_{T}^{K S}<10.0 \mathrm{GeV}$

- Weight with the $p_{T}$ Efficiency Correction

- Weight with the Number of Tracks per Jet Trigger Correction

- Weight with the Jet $E_{T}$ Trigger Correction

- Group into bins according to the range of the $p_{T}^{K S}$

\subsection{Comparing the Data with HERWIG+QFL Monte Carlo}

To derive the $\frac{1}{N_{j e t}} \frac{1}{p_{T}} \frac{d N_{K S}}{d p_{T}}$ and $\frac{1}{N_{j e t}} \frac{1}{p_{T}} \frac{d N_{t r a c k}}{d p_{T}}$ curves, the number of entries in each bin of a weighted background subtracted $p_{T}$ histogram is divided by the values of 
the total number of jets in a given jet $E_{T}$ range $(20-50,50-100$, and $100-150 \mathrm{GeV})$, the midpoint of the $p_{T}$ range, and the width of the $p_{T}$ range. These results are displayed in Figures 10.1-10.6. The data is denoted as points while the overlapping solid curves represent the HERWIG+QFL Monte Carlo results derived Chapter 9.

Similar to the $\frac{E}{N_{\text {event }}} \frac{d^{3} N_{K S}}{d^{3} p}$ spectrum in Chapter 5 , the $\frac{1}{N_{\text {jet }}} \frac{1}{p_{T}} \frac{d N_{K S}}{d p_{T}}$ and $\frac{1}{N_{\text {jet }}}$ $\frac{1}{p_{T}} \frac{d N_{t r a c k}}{d p_{T}}$ plots are fitted with a power law. Each curve here will be fitted from 1.5 to $10.0 \mathrm{GeV}$ on two consecutive occasions. At first 3 free parameters $\left(\mathrm{A}, \mathrm{n}, p_{0}\right)$ are used, then $p_{0}$ is fixed and the spectrum is refitted with only 2 free parameters $(A, n)$. This is done in order to simplify the error calculation. These parameters will be needed to compute the number of $K_{S}$ and tracks per jet as well as $\left\langle p_{T}\right\rangle$ for both $K_{S}$ and tracks in jets.

After fitting the $\frac{1}{N_{j e t}} \frac{1}{p_{T}} \frac{d N_{K S}}{d p_{T}}$ and $\frac{1}{N_{j e t}} \frac{1}{p_{T}} \frac{d N_{\text {track }}}{d p_{T}}$ plots, the power law with its known parameters are incorporated in the calculation of interesting quantities. To compute the number of $K_{S}\left(\frac{N_{K S}}{j e t}\right)$ and tracks per jet $\left(\frac{N_{t r a c k}}{j e t}\right)$, the power law multiplied by $p_{T}$ needs to be integrated. Moreover, as for the $\left\langle p_{T}\right\rangle$ for $K_{S}$ and tracks in jets, the ratio of the integral of the power law times the square of the $p_{T}$ and the integral of the power law multiplied by $p_{T}$ needs to be determined. The limits of integration are altered to generate quantities valid over different ranges of $p_{T}$. Equations 10.1 and 10.2 show the equations used to calculate $\frac{N_{K S}}{j e t}$ and $\frac{N_{\text {track }}}{\text { jet }}$ whereas equations 10.3 and 10.4 are implemented in the computation of the $<p_{T}>$ of the tracks and $K_{S}$. The equations are given below:

$$
\begin{aligned}
\frac{N_{K S}}{j e t} & =\int_{1.0 \mathrm{GeV}}^{10.0 \mathrm{GeV}} \frac{A p_{0}^{n} p_{T} d p_{T}}{\left(p_{T}+p_{0}\right)^{n}} \\
\frac{N_{\text {track }}}{j e t} & =\int_{1.0 \mathrm{GeV}}^{10.0 \mathrm{GeV}} \frac{A p_{0}^{n} p_{T} d p_{T}}{\left(p_{T}+p_{0}\right)^{n}}
\end{aligned}
$$




$$
\begin{gathered}
<p_{T}^{K S}>=\frac{\int_{1.0 \mathrm{GeV}}^{10.0 \mathrm{GeV}} \frac{A p_{0}^{n} p_{T}^{2} d p_{T}}{\left(p_{T}+p_{0}\right)^{n}}}{\int_{1.0 \mathrm{GeV}}^{10.0 \mathrm{GeV}} \frac{A p_{0}^{n} p_{T} d p_{T}}{\left(p_{T}+p_{0}\right)^{n}}} \\
<p_{T}^{\text {track }}>=\frac{\int_{1.0 \mathrm{GeV}}^{10.0 \mathrm{GeV}} \frac{A p_{0}^{n} p_{T}^{2} d p_{T}}{\left(p_{T}+p_{0}\right)^{n}}}{\int_{1.0 \mathrm{GeV} V}^{10.0 \mathrm{GeV} V} \frac{A p_{0}^{n} p_{p} d p_{T}}{\left(p_{T}+p_{0}\right)^{n}}} .
\end{gathered}
$$

In Tables 10.1 through 10.6, the $\frac{N_{K S}}{j e t}, \frac{N_{\text {track }}}{\text { jet }},\left\langle p_{T}^{K S}\right\rangle$, and $\left\langle p_{T}^{\text {track }}\right\rangle$ for all three jet $E_{T}$ ranges are listed for both the data and the HERWIG+QFL Monte Carlo with limits of integration from $1.0 \mathrm{GeV}$ to $10.0 \mathrm{GeV}$ and $1.5 \mathrm{GeV}$ to $10.0 \mathrm{GeV}$. The results of the data with limits of integration from $1.5 \mathrm{GeV}$ to $10.0 \mathrm{GeV}$ in these tables are consistent with those non-fitted numbers computed in Chapter 8 and Chapter 9.

For the number of tracks inside jets with $p_{T}$ between 1.5 and $10.0 \mathrm{GeV}$, the ratio between the results of the data and the HERWIG+QFL Monte Carlo grows from within $5 \%$ for $20-50 \mathrm{GeV}$ and $50-100 \mathrm{GeV}$ cases to almost $10 \%$ for the $100-150 \mathrm{GeV}$ jets. This analysis is not the first to notice the $10 \%$ difference between the tracks in jets in the data and the HERWIG+QFL Monte Carlo for high transverse energy jets.

Since the $K_{S}$ efficiency may be approximated by the product of two single track efficiencies, one might predict the discrepancy of the data and the HERWIG+QFL Monte Carlo for the production of the $K_{S}$ inside jets. Doing this, one is led to believe that the data and the HERWIG+QFL Monte Carlo for $K_{S}$ inside 20-50 $\mathrm{GeV}$ and $50-100 \mathrm{GeV}$ jets would be in agreement because the corresponding cases for the tracks inside jets in the data are consistent with the HERWIG+QFL Monte Carlo. Thus, the $K_{S}$ inside $100-150 \mathrm{GeV}$ jets would be predicted to have the data about $20 \%(90 \% * 90 \%=81 \%$, assuming reconstruction efficiency $\sim 100 \%)$ lower than the HERWIG+QFL Monte Carlo. 
Surprisingly, although the number of $K_{S}$ inside $20-50 \mathrm{GeV}$ jets in the data (with $p_{T}$ between 1.5 and $10.0 \mathrm{GeV}$ ) agrees to within $5 \%$ of the HERWIG+QFL Monte Carlo, the disagreement grows to approximately $20 \%$ and $35 \%$ for the $50-100 \mathrm{GeV}$ and $100-150 \mathrm{GeV}$ jets, respectively. The number of $K_{S}$ per jet in the data tends to be lower than what is expected from the HERWIG+QFL Monte Carlo. The disagreement is even $20 \%$ greater than what would be expected from a possible inaccuracy in the track efficiency in jets alone.

It is feasible that the data and the HERWIG+QFL Monte Carlo vary because detector efficiency issues, but even so, this is not believed to be the case. Instead, it more likely that the production of the $K_{S}$ inside jets for the HERWIG Monte Carlo is overestimated, especially at higher and higher jet $E_{T}$.

On the other hand, the $\left\langle p_{T}^{\text {track }}\right\rangle$ and $\left\langle p_{T}^{K S}\right\rangle$ agree to within $5 \%$ for all jet $E_{T}$ (with the exception of the 1.0-10.0 GeV case for $K_{S}$ where it is within $10 \%$ ). Consequently, the shape of the HERWIG+QFL Monte Carlo and the data are consistent for tracks and $K_{S}$ inside jets for all jet $E_{T}(20-150 \mathrm{GeV})$. Moreover, Figures 10.1-10.6 reflect these observations. Typically, the 1.0 to $10.0 \mathrm{GeV}$ case is within $5 \%$ of the 1.5 to $10.0 \mathrm{GeV}$ case, except for the $20-50 \mathrm{GeV}$ case where it is within $10 \%$ for the number of $K_{S}$.

\subsection{Comparing Fragmentation with Other Ex- periments}

One of the motivations of this analysis is to compare the fragmentation function and hadronization of jets from $1.8 \mathrm{TeV} p \bar{p}$ collisions with those of $e^{+} e^{-}$collisions at other center of mass energies. It is expected that just the type and the momenta 
of the outgoing partons determine the fragmentation function and hadronization, and that the fragmentation function might scale with the center of mass energy. Typically, the fragmentation function results from $e^{+} e^{-}$machines are quoted in terms of $x_{T}=\frac{p_{K S}}{p_{\text {beam }}}$ and charged kaons. It should be noted that the $p_{\text {beam }}$ variable should be $\frac{\sqrt{s}}{2}$, and center of mass collisions at $\sqrt{s}$ usually give rise to back-to-back jets at energies of $\frac{\sqrt{s}}{2}$. On the other hand, the interaction energy of partons in $p \bar{p}$ collisions is only a fraction of the total momenta of proton and antiproton carried by the interaction quarks. In this case, most of this energy for hard-scattering in the central region is given by the jet $E_{T}$. Likewise, most of the momenta of the $K_{S}$ in the central region consists of transverse momenta $p_{T}$. Thus, the fragmentation results in this analysis will be presented in terms of $x_{T}=\frac{p_{T}}{j e t E_{T}}$. In order to compare with the charged kaons production from $e^{+} e^{-}$collisions, this result needs to be multiplied by a factor of 2 because charged kaons production is assumed to be twice that of $K_{S}$ production.

There are a few of differences between the generation of the $p_{T}$ spectra and the fragmentation curves. First, the $K_{S}$ in jets are booked according to $\frac{p_{T}}{J e t E_{T}}$ instead of $p_{T}$. Also, just the $p_{T}$ efficiency and the jet $E_{T}$ trigger weights are applied. As for the jet $E_{T}$ trigger corrections for the fragmentation plots, the jet $E_{T}$ spectra is examined at sufficiently narrow intervals. Consequently, the jet $E_{T}$ of each desired range are adjusted to have a constant distribution. In particular, there are three plots for $K_{S}$ in jets for the $35-45 \mathrm{GeV}, 75-85 \mathrm{GeV}$, and $115-125 \mathrm{GeV}$ jet $E_{T}$ ranges.

The $K_{S}$ candidates are weighted prior to background subtraction, and the number of weighted background subtracted $K_{S}$ will be plotted against $x_{T}=\frac{p_{T}}{j e t E_{T}}$. Neither the number of tracks per jet trigger corrections nor subtractions of background rates in the generation of fragmentation spectra are included. These other 
corrections are left out because they are believed to small. To get the $\frac{p_{T}}{J e t E_{T}}$ per jet, each bin in the $\frac{p_{T}}{J e t E_{T}}$ curve is divided by the values of the total number of jets in the particular jet $E_{T}$ range $(35-45 \mathrm{GeV}, 75-85 \mathrm{GeV}$, and $115-125 \mathrm{GeV})$ and the width of the $\frac{p_{T}}{J e t E_{T}}$ range.

These results are shown in Figures 10.7-10.9 with the approximate $e^{+} e^{-}$results, [9] [77] [78] [79] [80] [81], overlapping the curves. Again, the shapes of the previous data are similar to this analysis although the Tevatron's points appear to be slightly lower. This could be evidence of scaling violations due to the greater collision energies at the Tevatron. To render greater detail, as the collision energy increases, the fragmentation curve is expected to shift to lower values of $x_{T}$. Clearly, this may case since the Tevatron's points appear to be lower. The results from all jet $E_{T}$ ranges $(20-50,50-100,100-150 \mathrm{GeV})$ are displayed together in Figure 10.10, and they overlap as they should since regardless of the jet $E_{T}$, all Tevatron fragmentation curves are generated at the same collision energy.

\subsection{Estimation of Systematics}

All errors quoted in this analysis are statistical. Although systematics has not been done in a rigorous sense, over the course of this analysis, a reliable estimation may be made. There are many potential sources of systematics: the variation of cuts, the particulars of the track embedding (such as incorporating the track density distribution), the fitting of the efficiency curves, the fitting of the mass curves, various implementations of trigger and efficiency corrections, and the fitting of the final spectra with a power law. The dominant systematic error is believed to arise from the fitting of the mass plus the background spectra, and it is on the order of 10\%. All of the other systematic errors would be expected to alter the results by 
around $5 \%$. The overall systematic error, with all of these contributing factors, is believed to be approximately $15 \%$.

\subsection{Conclusions}

In this thesis, the analysis of $K_{S}$ production properties inside jets began with studying $K_{S}$ production in Minimum Bias events. In order to compute the efficiencies, Monte Carlo $K_{S}$ are embedded into real Minimum Bias data events. The $K_{S}$ efficiency is found to be strongly correlated to the $p_{T}$ of the $K_{S}$. After implementing the efficiencies, the corrected $\mathrm{c} \tau$ of $K_{S}$ is measured to be $2.6882 \pm 0.0124$ $\mathrm{cm}$. This is within errors of the accepted value of $2.6786 \pm 0.0024 \mathrm{~cm}$. This implies that the generator and the simulator used for the track embedding are very reliable. In addition, the $\frac{E}{N_{\text {event }}} \frac{d^{3} N_{K S}}{d^{3} p}$ spectra as well as the invariant cross-section for $K_{S}$

production are within $5 \%$ of previous analyses. By fitting the $\frac{E}{N_{\text {event }}} \frac{d^{3} N_{K S}}{d^{3} p}$ spectra with a power law, the number of $K_{S}$ per eta $\frac{d N_{K S}}{d \eta}$ and the average $p_{T}$ of the $K_{S}$ $<p_{T}>$ are also computed. The $\frac{d N_{K S}}{d \eta}=0.129 \pm 0.005$ and the $\left\langle p_{T}\right\rangle=1.022 \pm 0.005$ for $K_{S}$ where $0.5 \mathrm{GeV}<p_{T}<10.0 \mathrm{GeV}$. Other calculations find these values to be in accord with previous publications.

After verifying the track-embedding Monte Carlo to be valid by studying the $K_{S}$ production in Minimum Bias events, $K_{S}$ and track production inside jets became the focus. The efficiencies are calculated by embedding Monte Carlo $K_{S}$ into jets in real data events, and the $K_{S}$ efficiency is strongly correlated to the $p_{T}$ of the $K_{S}$ as well as jet $E_{T}$. Furthermore, the jet triggers also effect both the jet $E_{T}$ spectra and the number of tracks per jet. By weighting $K_{S}$, tracks, and jets by correction factors, the $K_{S}$ and track production may be determined for a jet distribution which resembles that of the jet $E_{T}$ cross-section. Background corrections are made 
by tallying the number of $K_{S}$ or tracks inside an arbitrary cone away from all jets. Once this background is subtracted out, efficiency and trigger adjusted $\frac{1}{N_{j e t}}$ $\frac{1}{p_{T}} \frac{d N_{\text {track }}}{d p_{T}}$ and $\frac{1}{N_{j e t}} \frac{1}{p_{T}} \frac{d N_{K S}}{d p_{T}}$ spectra are determined for $K_{S}$ and tracks inside jets having $E_{T}$ ranges of $20-50 \mathrm{GeV}, 50-100 \mathrm{GeV}$, and $100-150 \mathrm{GeV}$.

Once the $\frac{1}{N_{j e t}} \frac{1}{p_{T}} \frac{d N_{\text {track }}}{d p_{T}}$ and $\frac{1}{N_{j e t}} \frac{1}{p_{T}} \frac{d N_{K S}}{d p_{T}}$ spectra are fitted with a power law, the number of $K_{S}$ per jet $\frac{N_{K S}}{j e t}$ and the number of tracks per jet $\frac{N_{\text {track }}}{\text { jet }}$ may be studied and compared with the HERWIG+QFL generator+simulator results. If the fragmentation and hadronization models are accurate, the agreement between the data and the Monte Carlo should be quite close. The HERWIG+QFL Monte Carlo predicts that both the $\frac{N_{K S}}{j e t}$ and $\frac{N_{\text {track }}}{\text { jet }}$ values increase with jet $E_{T}$. On the other hand, the data shows that although the $\frac{N_{\text {track }}}{j e t}$ quantity increases, the $\frac{N_{K S}}{j e t}$ value eventually becomes constant. This latter point is in stark contrast to the HERWIG+QFL predictions.

To render greater detail, in the data, for track production in jets, the $\frac{N_{\text {track }}}{j \text { jet }}$ quantity for $1.5<p_{T}<10.0 \mathrm{GeV}$ grows from $2.816 \pm 0.008,5.107 \pm 0.009,5.972 \pm 0.008$ for the $20-50 \mathrm{GeV}, 50-100 \mathrm{GeV}$, and $100-150 \mathrm{GeV}$ cases. This is inline with the expectations for the HERWIG+QFL values of $2.785 \pm 0.004,4.999 \pm 0.015,6.536 \pm 0.011$ for the $20-50 \mathrm{GeV}, 50-100 \mathrm{GeV}$, and $100-150 \mathrm{GeV}$ cases. Even at high jet $E_{T}$, the discrepancy is only $10 \%$. The HERWIG+QFL reproduces the data reasonably well.

As for the $\frac{N_{K S}}{j e t}$ variable, the data and HERWIG+QFL results diverge with jet $E_{T}$. For the data, the $\frac{N_{K S}}{j e t}$ quantities are $0.156 \pm 0.007$ for the $20-50 \mathrm{GeV}$ jets, $0.206 \pm 0.011$ for the $50-100 \mathrm{GeV}$ jets, and $0.199 \pm 0.011100-150 \mathrm{GeV}$ jets. Clearly, for jets above $50 \mathrm{GeV}$ jets, the $\frac{N_{K S}}{j e t}$ value is constant. The HERWIG+QFL values for $\frac{N_{K S}}{j e t}$ increase for all measured jet $E_{T}$ from $0.150 \pm 0.008$ for the $20-50 \mathrm{GeV}$ jets to $0.254 \pm 0.004$ for the $50-100 \mathrm{GeV}$ jets and to $0.308 \pm 0.005$ for the $100-150 \mathrm{GeV}$ 
jets. Thus, the HERWIG+QFL values are greater than those of the data by about $5 \%, 20 \%$, and $35 \%$ for the $20-50 \mathrm{GeV}, 50-100 \mathrm{GeV}$, and $100-150 \mathrm{GeV}$ cases. The differences are believed to be due to HERWIG overestimating $K_{S}$ production inside jets for the higher jet $E_{T}$ ranges.

In addition, although the number of $K_{S}$ per jet and the number of tracks increases for both the data and the Monte Carlo, at higher jet $E_{T}$, the $K_{S}$ production levels off for the data. As a result, 2.0 times the number of $K_{S}$ per track is almost constant for the Monte Carlo whereas it decreases substantially for the data. To give more details, the quantity of 2.0 times the number of $K_{S}$ per track decreases by approximately a factor of 0.60 from $0.111 \pm 0.001$ to $0.067 \pm 0.001$ for the $K_{S}$ and tracks inside the $20-50 \mathrm{GeV}$ and $100-150 \mathrm{GeV}$ jets. For the HERWIG Monte Carlo, this value is approximately constant, $0.109 \pm 0.001$ to $0.094 \pm 0.0003$ for the $20-50$ $\mathrm{GeV}$ and $100-150 \mathrm{GeV}$ cases, respectively.

The average $p_{T}$ of the tracks and the $K_{S},\left\langle p_{T}^{\text {track }}\right\rangle$ and $\left\langle p_{T}^{K S}\right\rangle$, are consistent for all three jet $E_{T}$ ranges. For the tracks with $1.5<p_{T}<10.0 \mathrm{GeV}$, the data predicts the $\left\langle p_{T}^{\text {track }}\right\rangle$ of $3.354 \pm 0.001 \mathrm{GeV}, 3.880 \pm 0.001 \mathrm{GeV}$, and $4.148 \pm 0.001 \mathrm{GeV}$ for the $20-50 \mathrm{GeV}, 50-100 \mathrm{GeV}$, and $100-150 \mathrm{GeV}$ cases. Moreover, for the $K_{S}$ with $1.5<p_{T}<10.0 \mathrm{GeV}$, the data predicts the $\left\langle p_{T}^{K S}>\right.$ of $3.809 \pm 0.026 \mathrm{GeV}, 4.348 \pm 0.031$ $\mathrm{GeV}$, and $4.458 \pm 0.036 \mathrm{GeV}$ for the $20-50 \mathrm{GeV}, 50-100 \mathrm{GeV}$, and $100-150 \mathrm{GeV}$ cases. Over all measured jet $E_{T}$ ranges, the values for $\left\langle p_{T}^{\text {track }}\right\rangle$ and $\left\langle p_{T}^{K S}\right\rangle$ increase with jet $E_{T}$, and the data and the HERWIG+QFL Monte Carlo are within a few percent of each other. Consequently, the fragmentation model in HERWIG predictions are valid.

In contrasting the fragmentation functions of the Tevatron with those from $e^{+} e^{-}$ experiments, the overall shapes of the fragmentation distributions are in fair agree- 
ment. However, in general, the Tevatron data is lower than the $e^{+} e^{-}$data. The fragmentation spectra for all of the jet $E_{T}$ ranges overlap for the Tevatron, and this indicates that fragmentation functions for the Tevatron are independent of jet $E_{T}$.

\subsection{Overview}

Since quarks and gluons have never been observed in isolation, various fragmentation models have attempted to address how these partons are converted into mesons and baryons. These groups of hadrons directed in region a phase space produced from a fragmenting parton are called "jets". Through the study of particle production within jets, greater insight to the particulars of the fragmentation process may be tested. Most of the fragmentation data comes from $e^{+} e^{-}$collisions, and these theories are also applied to those from $p \bar{p}$ collisions. To do so, many models assume that only the type and kind of outgoing parton from an interaction, rather than the process itself, governs the fragmentation process. In particular, an outgoing parton from a hard-scattering process will shower into other partons, and this is explained with perturbative QCD down to an energy scale of around a few hundred MeV.

At this point, in order to describe how groups of gluons and quarks are transformed into mesons and baryons, various hadronization models must be implemented. There are two major types of hadronization models: the string and the cluster model. The string model is based upon the breaking of strong force lines from separating quarks into quark-antiquark pairs, and the cluster model consists of grouping and decaying clusters of quarks and gluons in regions of phase space. Both of these models predict that strange quark production (and particles containing strange quarks) will be suppressed. In this analysis, charged particle and strangeness production in the data will be compared with HERWIG, an event gen- 
erator based upon the cluster model. Moreover, strangeness production will be contrasted with $e^{+} e^{-}$experiments. Due to the higher mass of the strange quarks, studies in strange quark production reveal additional information as to how partons are converted into hadrons that is not otherwise provided through the examination of the fragmentation of only the lighter up and down quarks.

It is found that HERWIG generates too many tracks and $K_{S}$ inside high $E_{T}$ jets. However, the discrepancy between the data and HERWIG may not all be attributed to an overall discrepancy in track production inside jets. Through comparing $K_{S}$ fragmentation functions from $p \bar{p}$ collisions with those of other experiments, the premise of fragmentation depending only upon the type and the energy of the outgoing parton is tested. In addition, possible scaling violations may be observed. The Tevatron $p \bar{p}$ results are below those from $e^{+} e^{-}$machines. Also, the shapes of the fragmentations is in fair agreement, but conclusions as to the existence of scaling violations are indeterminate.

This study indicates that the HERWIG cluster hadronization model needs to be adjusted in regard to strangeness and track production for high $E_{T}$ jets, though $<p_{T}>$ of $K_{S}$ and tracks inside jets are consistent. 
Table 10.1: The $\frac{N_{\text {particle }}}{\text { jet }}$ and $\left\langle p_{T}\right\rangle$ values for tracks and $K_{S}$ inside $20-50 \mathrm{GeV}$ jets in the data. Systematic errors are not included.

\begin{tabular}{|c|c|c|}
\hline & tracks in 20-50 Jets & $K_{S}$ in 20-50 Jets \\
\hline Fit Range & $1.5<p_{T}<10.0 \mathrm{GeV}$ & $1.5<p_{T}<10.0 \mathrm{GeV}$ \\
\hline Function Fitted & $\frac{A p_{0}^{n}}{\left(p_{0}+p_{T}\right)^{n}}$ & $\frac{A p_{0}^{n}}{\left(p_{0}+p_{T}\right)^{n}}$ \\
\hline Resulting & $A=10.313 \pm 0.022$ & $A=0.155 \pm 0.005$ \\
Fit Parameters & $n=5.345 \pm 0.003$ & $n=6.845 \pm 0.076$ \\
& $p_{0}=2.826($ fixed $)$ & $p_{0}=6.583($ fixed $)$ \\
\hline$\frac{N_{\text {particle }} \text { from } 1.0-10.0 \mathrm{GeV}}{\text { jet }}$ fixt & $3.725 \pm 0.010$ & $0.185 \pm 0.008$ \\
$\frac{N_{\text {particle }} \text { from } 1.5-10.0 \mathrm{GeV}}{\text { jet }}$ from 1.0-10.0 GeV & $2.816 \pm 0.008$ & $0.156 \pm 0.007$ \\
$\left\langle p_{T}>\right.$ from & $2.838 \pm 0.001$ & $3.404 \pm 0.029$ \\
$\left\langle p_{T}>\right.$ from 1.5-10.0 GeV & $3.354 \pm 0.001$ & $3.809 \pm 0.026$ \\
\hline
\end{tabular}

Table 10.2: The $\frac{N_{\text {particle }}}{\text { jet }}$ and $\left\langle p_{T}>\right.$ values for tracks and $K_{S}$ inside $20-50 \mathrm{GeV}$ jets in HERWIG Monte Carlo. Systematic errors are not included.

\begin{tabular}{|c|c|c|}
\hline & tracks in 20-50 Jets & $K_{S}$ in 20-50 Jets \\
\hline Fit Range & $1.5<p_{T}<10.0 \mathrm{GeV}$ & $1.5<p_{T}<10.0 \mathrm{GeV}$ \\
\hline Function Fitted & $\frac{A p_{0}^{n}}{\left(p_{0}+p_{T}\right)^{n}}$ & $\frac{A p_{0}^{n}}{\left(p_{0}+p_{T}\right)^{n}}$ \\
\hline Resulting & $A=7.985 \pm 0.048$ & $A=0.801 \pm 0.031$ \\
Fit Parameters & $n=5.821 \pm 0.009$ & $n=3.606 \pm 0.029$ \\
& $p_{0}=3.493($ fixed $)$ & $p_{0}=1.296($ fixed $)$ \\
\hline$\frac{N_{\text {particle }} \text { from 1.0-10.0 GeV }}{\text { jet }}$ from & $3.625 \pm 0.028$ & $0.194 \pm 0.010$ \\
$\frac{N_{\text {particle }} \text { from } 1.5-10.0 \mathrm{GeV}}{\text { jet }}$ from $1.0-10.0 \mathrm{GeV}$ & $2.785 \pm 0.023$ & $0.150 \pm 0.008$ \\
$\left\langle p_{T}>\right.$ from & $2.883 \pm 0.004$ & $3.137 \pm 0.024$ \\
$\left\langle p_{T}>\right.$ from 1.5-10.0 GeV & $3.378 \pm 0.004$ & $3.694 \pm 0.021$ \\
\hline
\end{tabular}


Table 10.3: The $\frac{N_{\text {particle }}}{j e t}$ and $\left\langle p_{T}>\right.$ values for tracks and $K_{S}$ inside $50-100 \mathrm{GeV}$ jets in the data. Systematic errors are not included.

\begin{tabular}{|c|c|c|}
\hline & tracks in 50-100 Jets & $K_{S}$ in 50-100 Jets \\
\hline Fit Range & $1.5<p_{T}<10.0 \mathrm{GeV}$ & $1.5<p_{T}<10.0 \mathrm{GeV}$ \\
\hline Function Fitted & $\frac{A p_{0}^{n}}{\left(p_{0}+p_{T}\right)^{n}}$ & $\frac{A p_{0}^{n}}{\left(p_{0}+p_{T}\right)^{n}}$ \\
\hline Resulting & $A=16.448 \pm 0.022$ & $A=0.163 \pm 0.006$ \\
Fit Parameters & $n=3.503 \pm 0.001$ & $n=3.805 \pm 0.049$ \\
& $p_{0}=1.511($ fixed $)$ & $p_{0}=3.259($ fixed $)$ \\
\hline$\frac{N_{\text {particle }}}{\text { jet }}$ from $1.0-10.0 \mathrm{GeV}$ & $6.351 \pm 0.011$ & $0.236 \pm 0.012$ \\
$\frac{N_{\text {particle }} \text { from } 1.5-10.0 \mathrm{GeV}}{\text { jet }}$ & $5.107 \pm 0.009$ & $0.206 \pm 0.011$ \\
$\left\langle p_{T}\right\rangle$ from $1.0-10.0 \mathrm{GeV}$ & $3.362 \pm 0.001$ & $3.961 \pm 0.035$ \\
$\left\langle p_{T}>\right.$ from $1.5-10.0 \mathrm{GeV}$ & $3.880 \pm 0.001$ & $4.348 \pm 0.031$ \\
\hline
\end{tabular}

Table 10.4: The $\frac{N_{\text {particle }}}{\text { jet }}$ and $\left\langle p_{T}\right\rangle$ values for tracks and $K_{S}$ inside $50-100 \mathrm{GeV}$ Jets in HERWIG Monte Carlo. Systematic errors are not included.

\begin{tabular}{|c|c|c|}
\hline & tracks in $50-100 \mathrm{Jets}$ & $K_{S}$ in $50-100 \mathrm{Jets}$ \\
\hline Fit Range & $1.5<p_{T}<10.0 \mathrm{GeV}$ & $1.5<p_{T}<10.0 \mathrm{GeV}$ \\
\hline Function Fitted & $\frac{A p_{0}^{n}}{\left(p_{0}+p_{T}\right)^{n}}$ & $\frac{A p_{0}^{n}}{\left(p_{0}+p_{T}\right)^{n}}$ \\
\hline Resulting & $A=13.639 \pm 0.029$ & $A=0.309 \pm 0.003$ \\
Fit Parameters & $n=3.562 \pm 0.002$ & $n=3.591 \pm 0.011$ \\
& $p_{0}=1.669($ fixed $)$ & $p_{0}=2.422($ fixed $)$ \\
\hline$\frac{N_{\text {particle }}}{\text { jet }}$ from $1.0-10.0 \mathrm{GeV}$ & $6.162 \pm 0.017$ & $0.298 \pm 0.004$ \\
$\frac{N_{\text {particle }} \text { from } 1.5-10.0 \mathrm{GeV}}{\text { jet }}$ & $4.999 \pm 0.015$ & $0.254 \pm 0.004$ \\
$\left\langle p_{T}\right\rangle$ from $1.0-10.0 \mathrm{GeV}$ & $3.407 \pm 0.001$ & $3.774 \pm 0.009$ \\
$\left\langle p_{T}\right\rangle$ from 1.5-10.0 GeV & $3.910 \pm 0.001$ & $4.204 \pm 0.007$ \\
\hline
\end{tabular}


Table 10.5: The $\frac{N_{\text {particle }}}{\text { jet }}$ and $\left\langle p_{T}>\right.$ values for tracks and $K_{S}$ inside $100-150 \mathrm{GeV}$ Jets in the data. Systematic errors are not included.

\begin{tabular}{|c|c|c|}
\hline & tracks in 100-150 Jets & $K_{S}$ in 100-150 Jets \\
\hline Fit Range & $1.5<p_{T}<10.0 \mathrm{GeV}$ & $1.5<p_{T}<10.0 \mathrm{GeV}$ \\
\hline Function Fitted & $\frac{A p_{0}^{n}}{\left(p_{0}+p_{T}\right)^{n}}$ & $\frac{A p_{0}^{n}}{\left(p_{0}+p_{T}\right)^{n}}$ \\
\hline Resulting & $A=13.547 \pm 0.014$ & $A=0.084 \pm 0.003$ \\
Fit Parameters & $n=3.107 \pm 0.001$ & $n=5.749 \pm 0.093$ \\
& $p_{0}=1.419($ fixed $)$ & $p_{0}=7.815($ fixed $)$ \\
\hline$\frac{N_{\text {particle }} \text { from } 1.0-10.0 \mathrm{GeV}}{\text { jet }}$ from & $7.161 \pm 0.009$ & $0.221 \pm 0.012$ \\
$\frac{N_{\text {particle }}}{\text { jet }}$ from $1.5-10.0 \mathrm{GeV}$ & $5.972 \pm 0.008$ & $0.199 \pm 0.011$ \\
$<p_{T}>$ from $1.0-10.0 \mathrm{GeV}$ & $3.666 \pm 0.001$ & $4.135 \pm 0.040$ \\
$<p_{T}>$ from $1.5-10.0 \mathrm{GeV}$ & $4.148 \pm 0.001$ & $4.458 \pm 0.036$ \\
\hline
\end{tabular}

Table 10.6: The $\frac{N_{\text {particle }}}{\text { jet }}$ and $\left\langle p_{T}>\right.$ values for tracks and $K_{S}$ inside $100-150 \mathrm{GeV}$ jets in HERWIG Monte Carlo. Systematic errors are not included.

\begin{tabular}{|c|c|c|}
\hline & tracks in 100-150 Jets & $K_{S}$ in 100-150 Jets \\
\hline Fit Range & $1.5<p_{T}<10.0 \mathrm{GeV}$ & $1.5<p_{T}<10.0 \mathrm{GeV}$ \\
\hline Function Fitted & $\frac{A p_{0}^{n}}{\left(p_{0}+p_{T}\right)^{n}}$ & $\frac{A p_{0}^{n}}{\left(p_{0}+p_{T}\right)^{n}}$ \\
\hline Resulting & $A=17.172 \pm 0.023$ & $A=0.383 \pm 0.004$ \\
Fit Parameters & $n=2.994 \pm 0.001$ & $n=2.947 \pm 0.009$ \\
& $p_{0}=1.239($ fixed $)$ & $p_{0}=1.717($ fixed $)$ \\
\hline$\frac{N_{\text {particle }} \text { from 1.0-10.0 GeV }}{\text { jet }}$ fixe & $7.866 \pm 0.012$ & $0.355 \pm 0.006$ \\
$\frac{N_{\text {particle }} \text { from 1.5-10.0 GeV }}{\text { jet }}$ from 1.0-10.0 GeV & $6.536 \pm 0.011$ & $0.308 \pm 0.005$ \\
$\left\langle p_{T}\right\rangle$ from & $3.664 \pm 0.001$ & $3.987 \pm 0.009$ \\
$\left\langle p_{T}\right\rangle$ from $1.5-10.0 \mathrm{GeV}$ & $4.156 \pm 0.001$ & $4.411 \pm 0.008$ \\
\hline
\end{tabular}




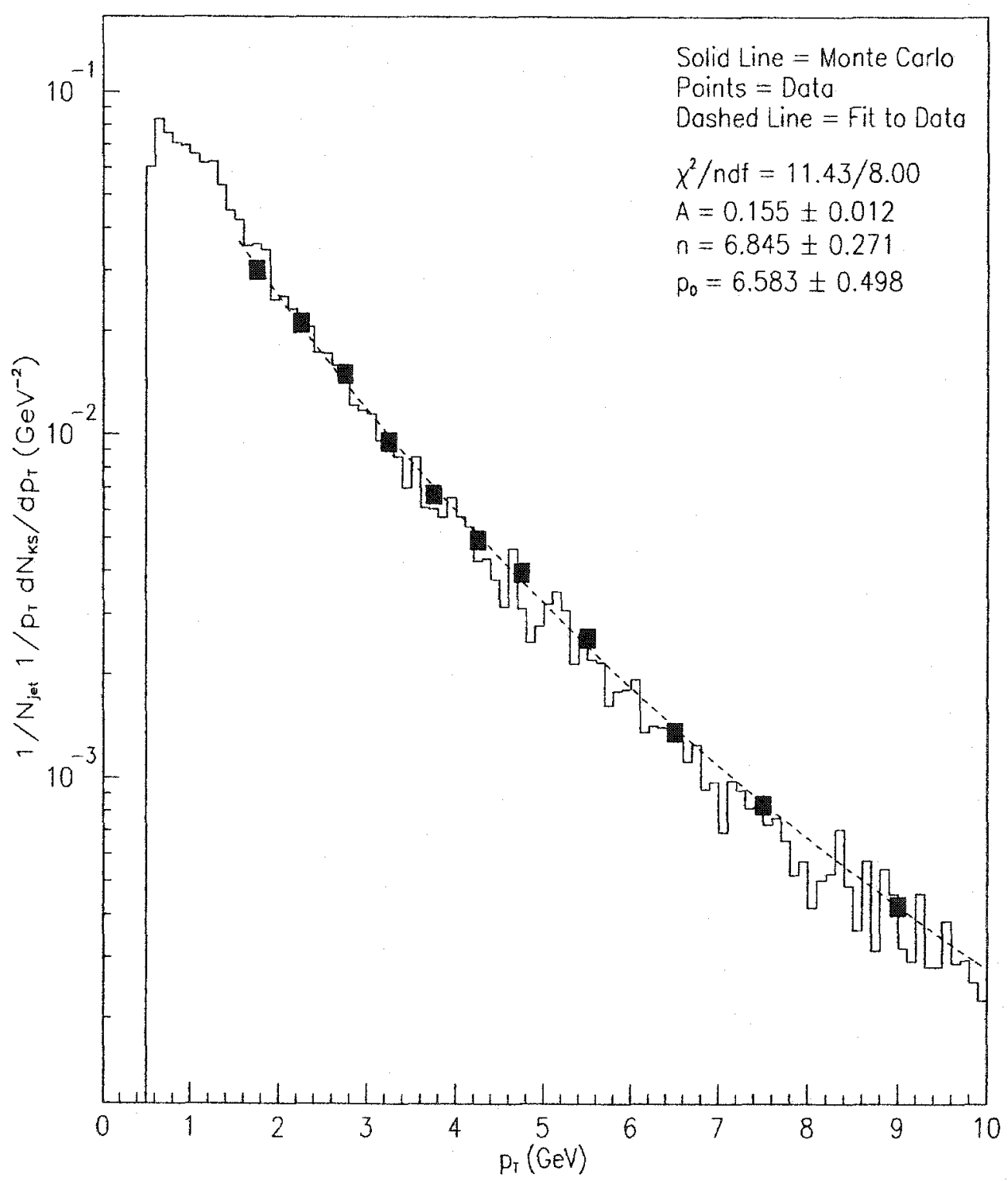

$1 / N_{\text {jet }} 1 / p_{\mathrm{T}} d N_{\mathrm{kS}} / d p_{\mathrm{T}}$ vs. $p_{\mathrm{T}}$ for $20-50 \mathrm{GeV}$ Jets

Figure 10.1: The $\frac{1}{N_{j e t}} \frac{1}{p_{T}} \frac{d N_{K S}}{d p_{T}}$ spectrum $K_{S}$ inside 20-50 GeV jets. Both the data and the Monte Carlo have been corrected for trigger and background effects. The errors on the HERWIG Monte Carlo are 0.0016, 0.0003 , and 0.0002 at $2.0 \mathrm{GeV}$, $5.0 \mathrm{GeV}$, and $7.0 \mathrm{GeV}$, respectively. Systematic errors are not included. 


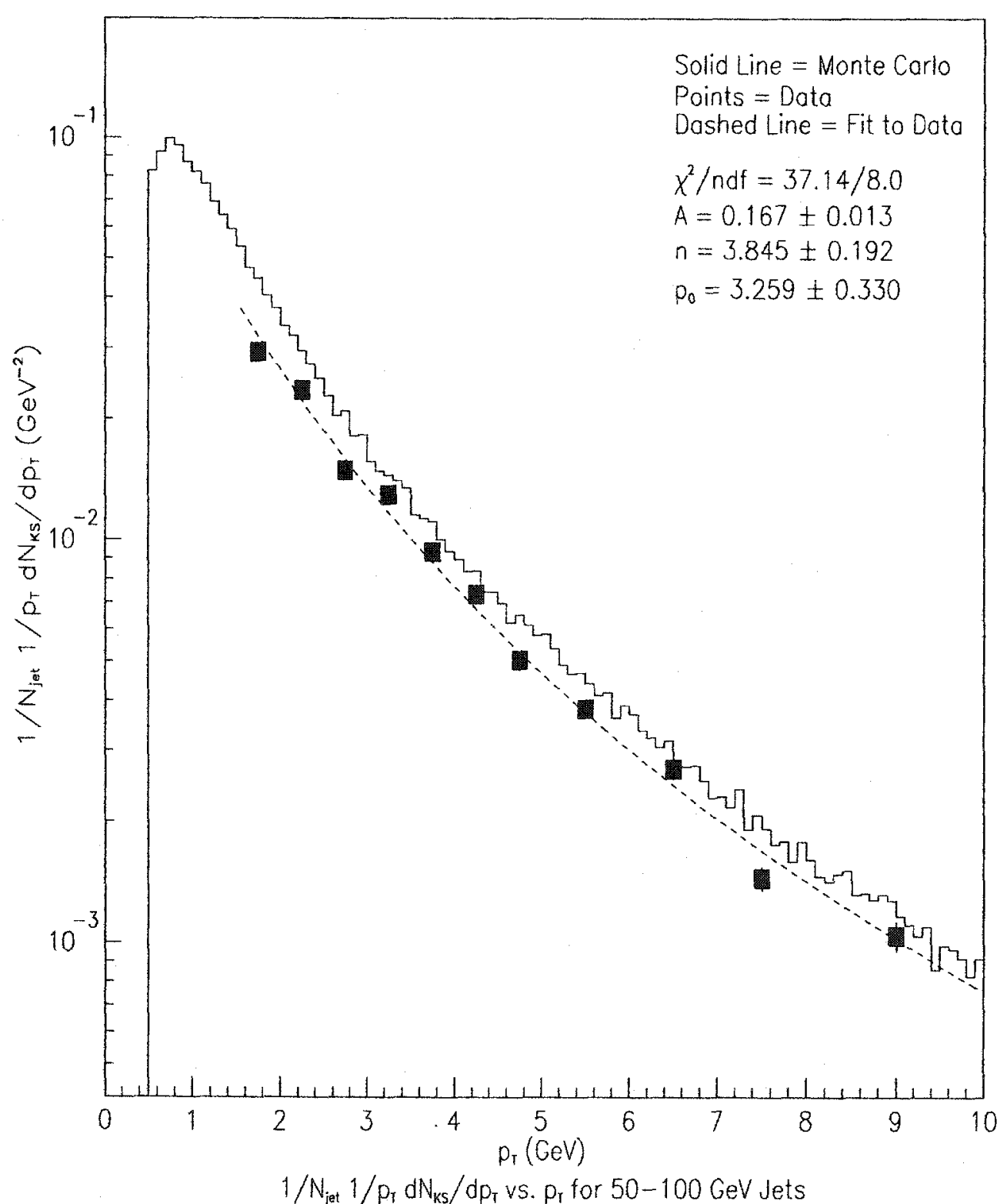

Figure 10.2: The $\frac{1}{N_{\text {jet }}} \frac{1}{p_{T}} \frac{d N_{K S}}{d p_{T}}$ spectrum for $K_{S}$ inside $50-100 \mathrm{GeV}$ jets. Both the data and the Monte Carlo have been corrected for trigger and background effects. The errors on the HERWIG Monte Carlo are 0.0009, 0.0002, and 0.0001 at $2.0 \mathrm{GeV}$, $5.0 \mathrm{GeV}$, and $7.0 \mathrm{GeV}$, respectively. Systematic errors are not included. 


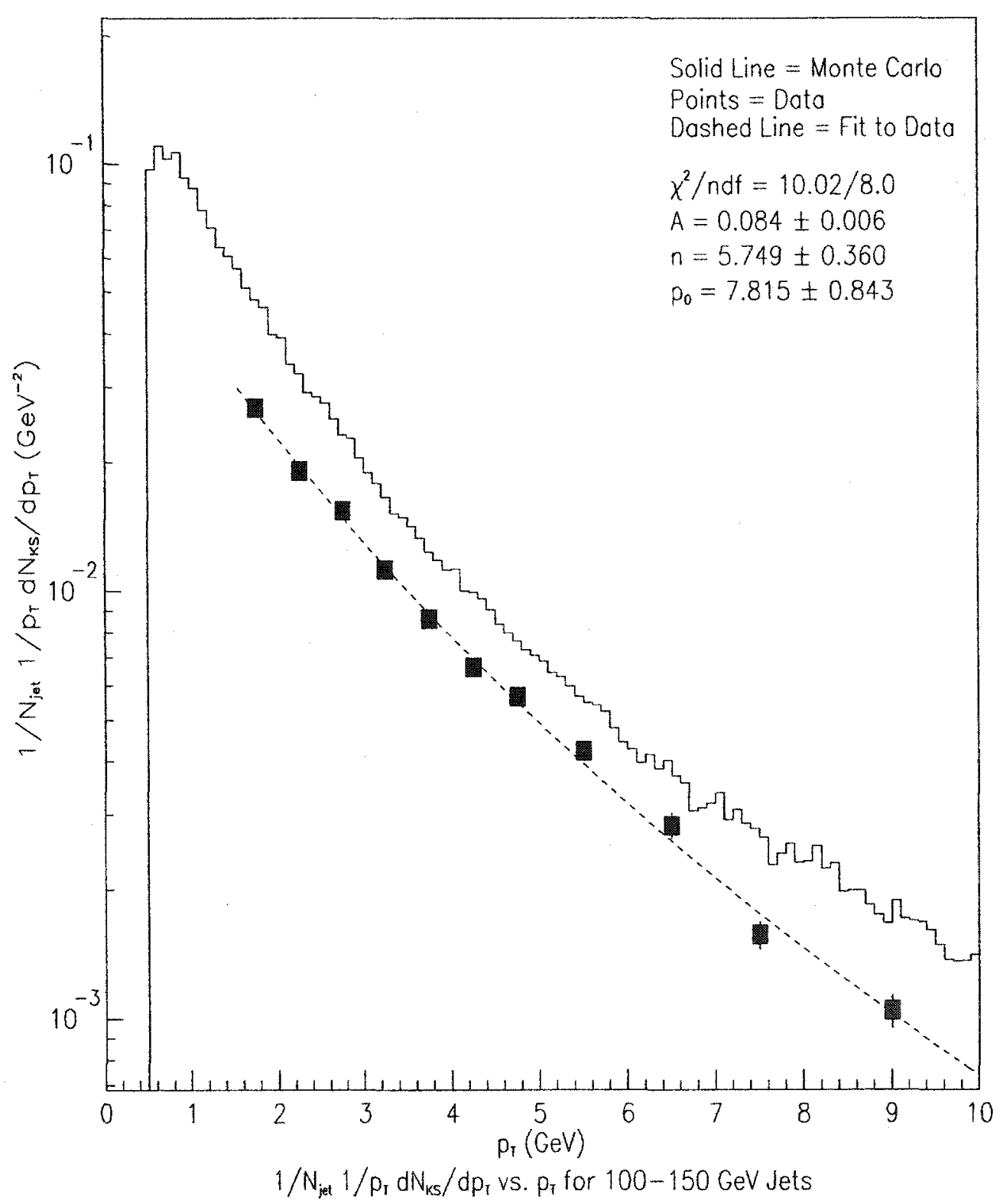

Figure 10.3: The $\frac{1}{N_{j e t}} \frac{1}{p_{T}} \frac{d N_{K S}}{d p_{T}}$ spectrum for $K_{S}$ inside $100-150 \mathrm{GeV}$ jets. Both the data and the Monte Carlo have been corrected for trigger and background effects. The errors on the HERWIG Monte Carlo are $0.0009,0.0002$, and 0.0001 at $2.0 \mathrm{GeV}$, $5.0 \mathrm{GeV}$, and $7.0 \mathrm{GeV}$, respectively. Systematic errors are not included. 


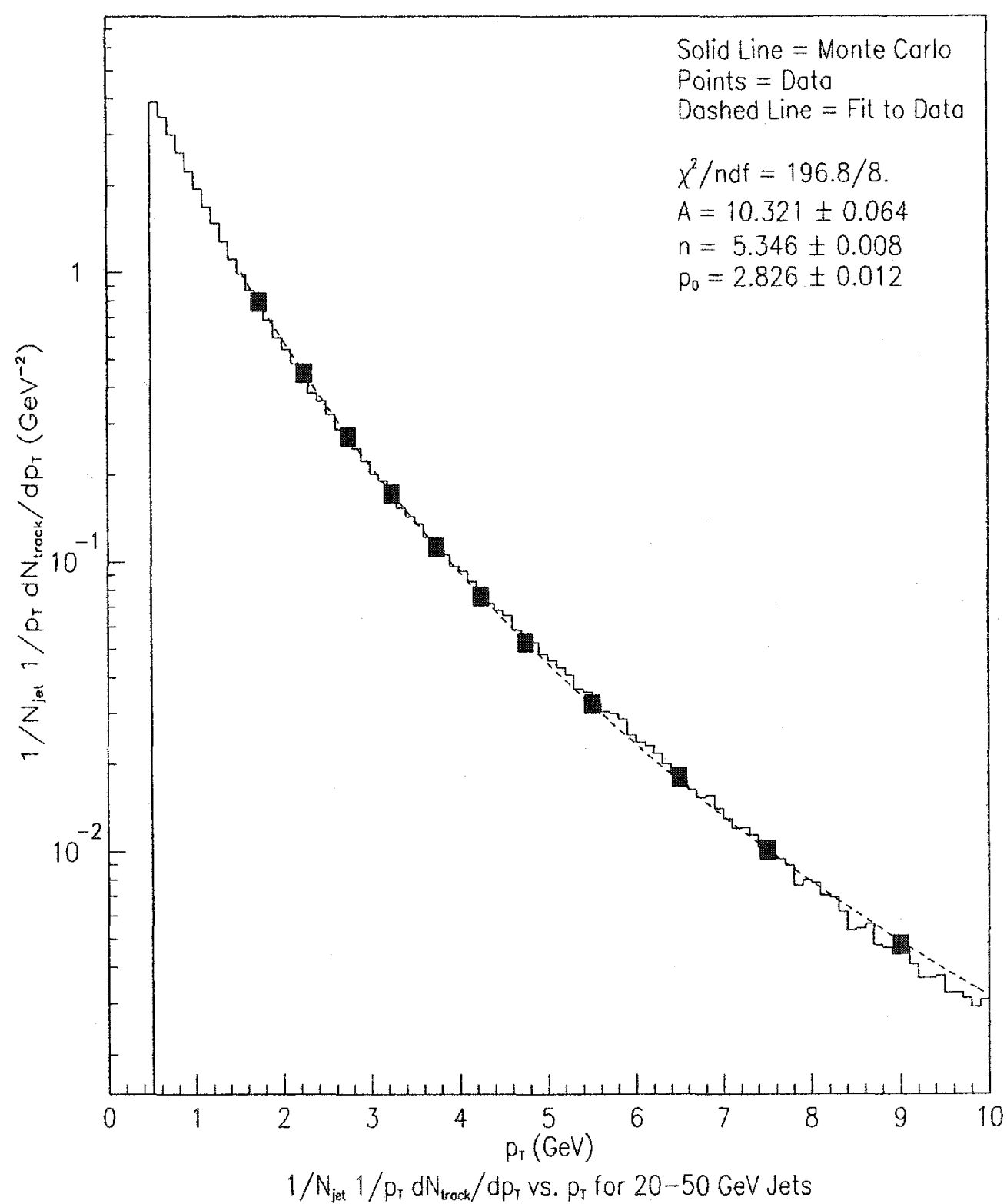

Figure 10.4: The $\frac{1}{N_{j e t}} \frac{1}{p_{T}} \frac{d N_{\text {track }}}{d p_{T}}$ spectrum for tracks inside $20-50 \mathrm{GeV}$ jets. Both the data and the Monte Carlo have been corrected for trigger and background effects. The errors on the HERWIG Monte Carlo are $0.0065,0.0011$, and 0.0005 at $2.0 \mathrm{GeV}, 5.0 \mathrm{GeV}$, and $7.0 \mathrm{GeV}$, respectively. Systematic errors are not included. 


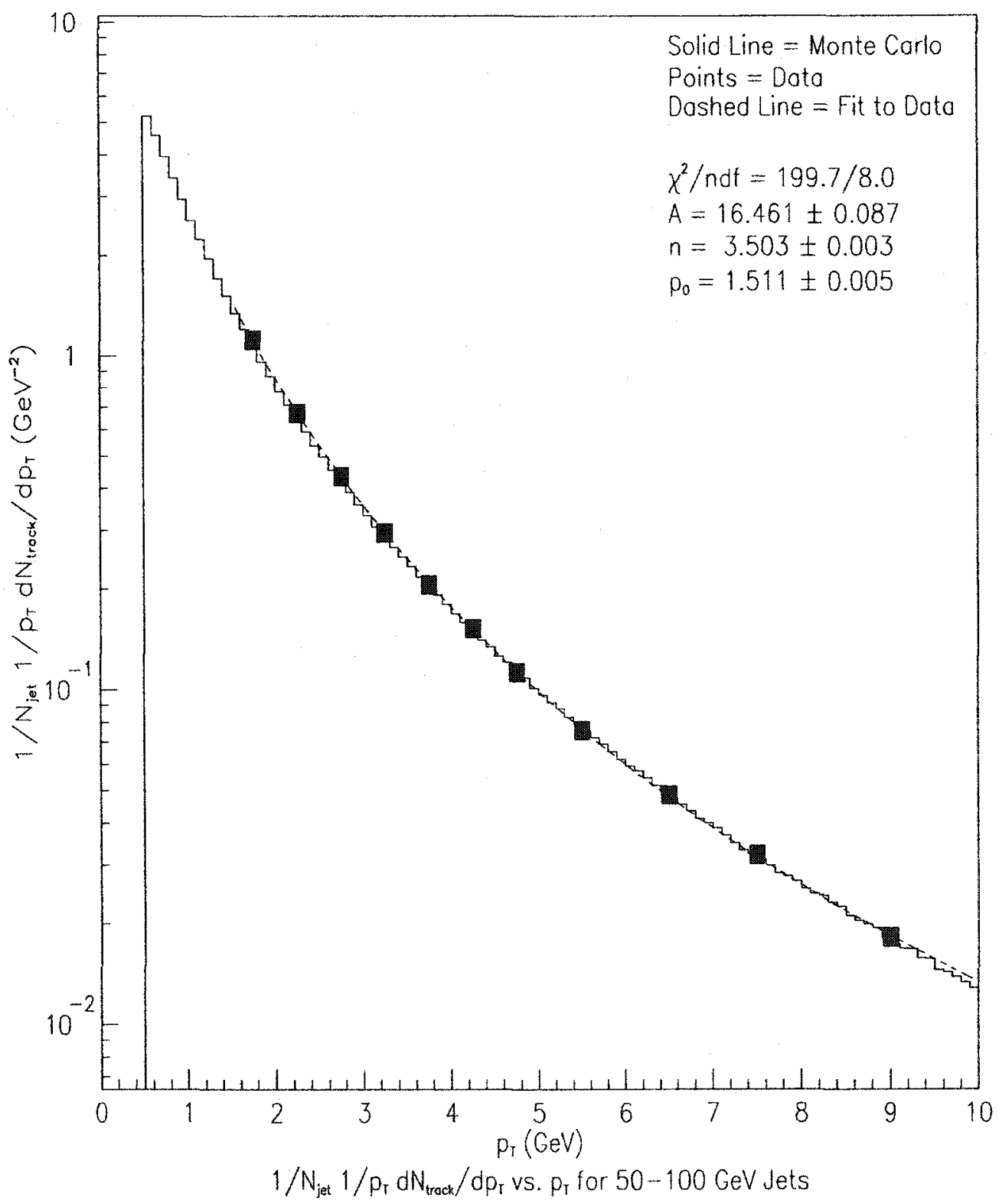

Figure 10.5: The $\frac{1}{N_{j e t}} \frac{1}{p_{T}} \frac{d N_{\text {track }}}{d p_{T}}$ spectrum for tracks inside $50-100 \mathrm{GeV}$ jets. Both the data and the Monte Carlo have been corrected for trigger and background effects. The errors on the HERWIG Monte Carlo are $0.0034,0.0007$, and 0.0004 at $2.0 \mathrm{GeV}, 5.0 \mathrm{GeV}$, and $7.0 \mathrm{GeV}$, respectively. Systematic errors are not included. 


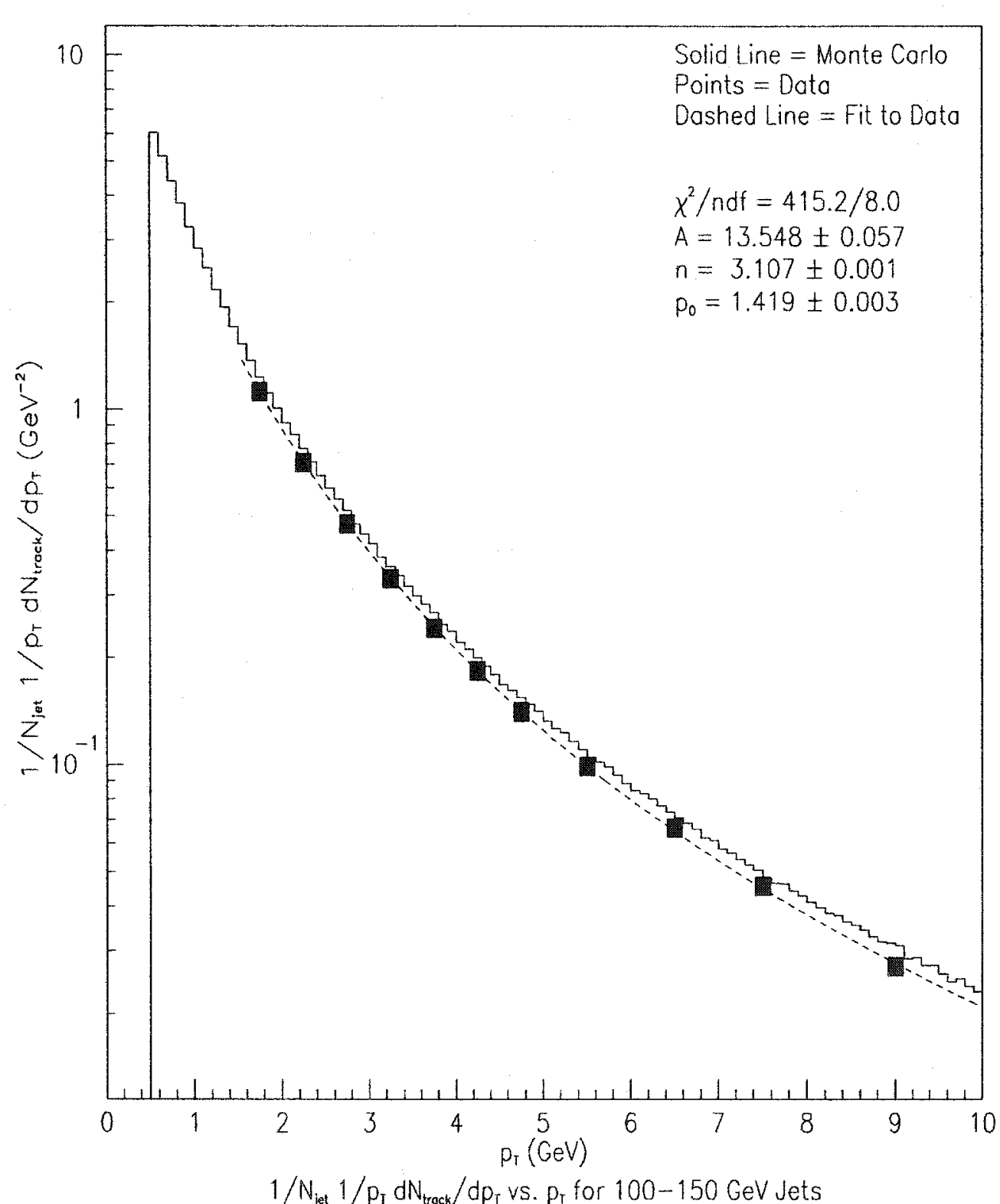

Figure 10.6: The $\frac{1}{N_{\text {jet }}} \frac{1}{p_{T}} \frac{d N_{\text {track }}}{d p_{T}}$ spectrum for tracks inside $100-150 \mathrm{GeV}$ jets. Both the data and the Monte Carlo have been corrected for trigger and background effects. The errors on the HERWIG Monte Carlo are $0.0039,0.0009$, and 0.0005 at $2.0 \mathrm{GeV}, 5.0 \mathrm{GeV}$, and $7.0 \mathrm{GeV}$, respectively. Systematic errors are not included. 


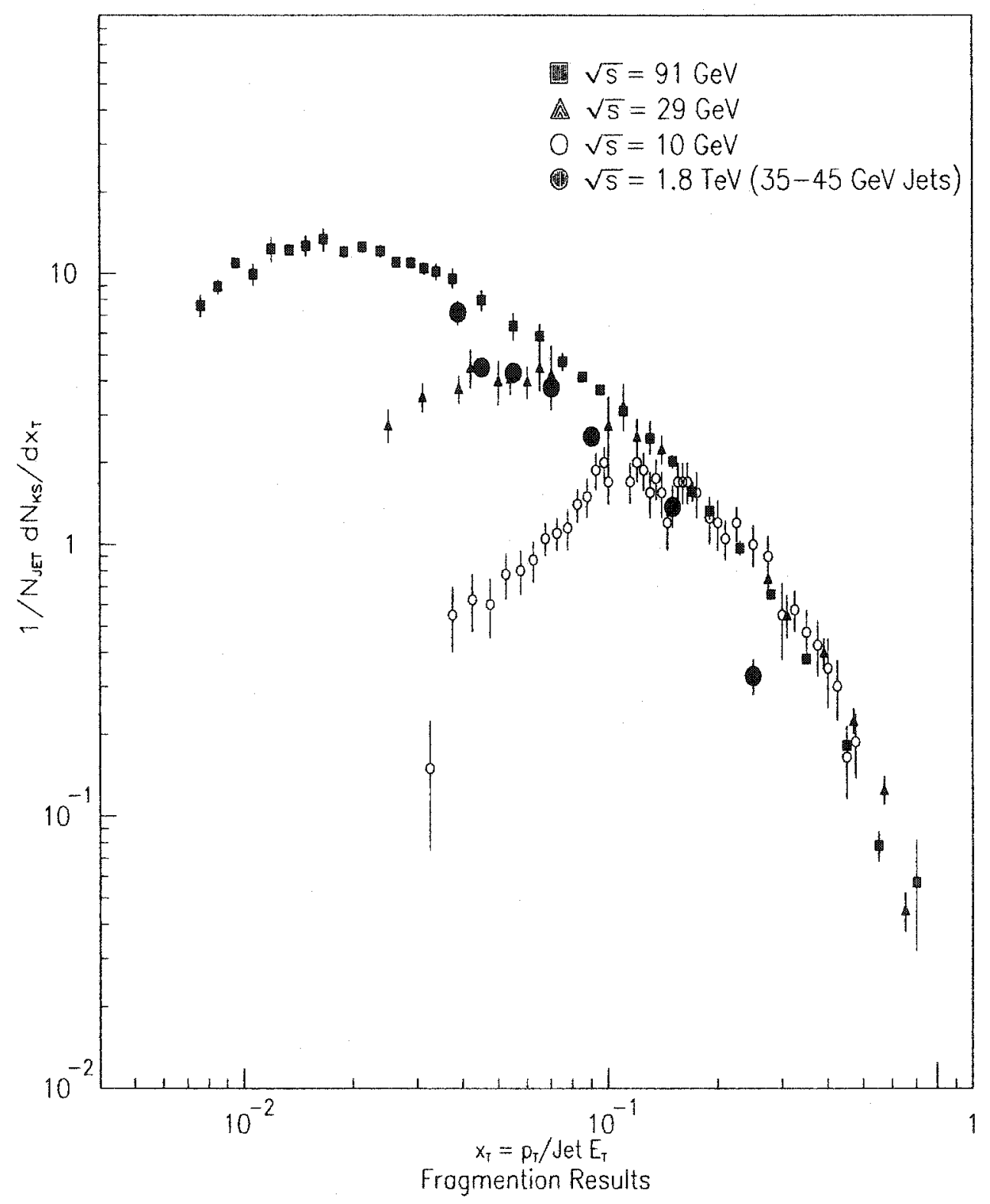

Figure 10.7: The fragmentation spectrum $K_{S}$ inside $20-50 \mathrm{GeV}$ jets. The solid circular symbols are the $p \bar{p}$ results whereas the triangles, the squares, and hollow circles represent the results from $e^{+} e^{-}$collisions. The data has been corrected for the trigger effect. Systematic errors are not included. 


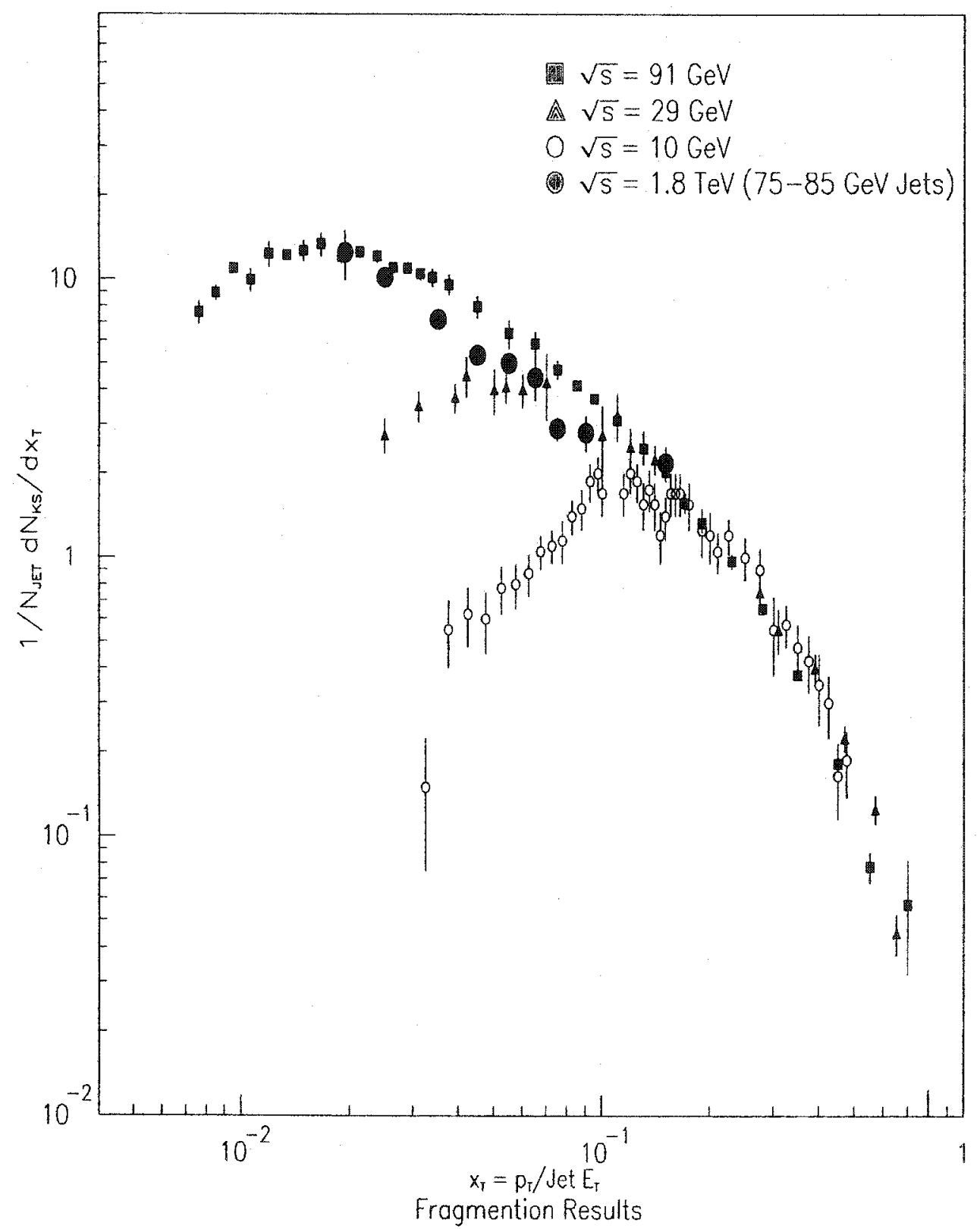

Figure 10.8: The fragmentation spectrum $K_{S}$ inside $50-100 \mathrm{GeV}$ jets. The solid circular symbols are the $p \bar{p}$ results whereas the triangles, the squares, and hollow circles represent the results from $e^{+} e^{-}$collisions. The data has been corrected for the trigger effect. Systematic errors are not included. 




Figure 10.9: The fragmentation spectrum $K_{S}$ inside $100-150 \mathrm{GeV}$ jets. The solid circular symbols are the $p \bar{p}$ results whereas the triangles, the squares, and hollow circles represent the results from $e^{+} e^{-}$collisions. The data has been corrected for the trigger effect. Systematic errors are not included. 




Figure 10.10: The fragmentation spectrum tracks inside 20-50,50-100,100-150 $\mathrm{GeV}$ jets. The data has been corrected for the trigger effect. Systematic errors are not included. 


\section{Bibliography}

[1] G.D. Rochester and C.C. Butler, "Evidence for the Existence of New Unstable Elementary Particles", Nature 160:855-857, 1947.

[2] C. Powell et al., "Observations with Electron Sensitive Plates Exposed to Cosmic Radiation", Nature 163:82, 1949.

[3] David Griffiths, "Introduction to Elementary Particles", John Wiley \& Sons, 1987

[4] M. Gell-Mann, Phys. Lett. 8, 218(1964); G. Zweig, CERN-8419-TH-412 (1964).

[5] B.R. Martin, G. Shaw, "Particle Physics", John Wiley \& Sons, 1992.

[6] D.Cronin-Hennessy, CDF thesis - "Tests of perturbative QCD in W+ jets events produced in $\sqrt{s}=1.8 \mathrm{TeV} \bar{p} p$ collisions", 1997 .

[7] J.R.Dittmann, CDF thesis - "Measurement of the $\mathrm{W}+\leq 1$ jet cross section in proton-antiproton collisions at $\sqrt{s}=1.8 \mathrm{TeV}, 1998$.

[8] M. Brozovic, CDF thesis - "Studies of the WZ Production Associated with Direct Photons, 2002.

[9] C. Caso et al. (Particle Data Group), The European Physical Journal C3(1998)

[10] Leslie S. Groer, CDF thesis - "A Search for Charged Higgs Boson Decays of the Top Quark Using Hadronic Decays of the Tau Lepton, in Proton-Antiproton Collisions at $\sqrt{s}=1.8 \mathrm{TeV}$ at CDF, 1998.

[11] Michael Schmelling, "Status of the Strong Coupling Constant", Plenary talk given at the XXVIII Intern. Conf. on High Energy Physics, Warsaw, July 25-31, 1996.

[12] W. Bardeen et al., Phys. Rev. D. 18, 3998 (1978).

[13] CCFR Collaboration: W.G. Seligman et al., Phys. Rev. Lett. 79, 1213 (1997); NMC Collaboration: M. Arneodo et al., Phys. Lett. B309, 222 (1993); L.W. Whitlow, Ph.D. thesis, SLAC Report 357 (1990). 
[14] S. L. Glashow et al., Phys. Rev. D2, 1285(1970); S. Weinberg, Phys. Rev. Lett. 19, 1264 (1967); A. Salam, p. 367 of Elementary Particle Theory, Almquist and Wiksells, Stockholm (1969).

[15] G. Arnison et al., Phys. Lett. 122B, 103 (1983).

[16] M. Banner et al, Phys. Lett. B 122, 476 (1983).

[17] G. Arnison et al., Phys. Lett. 126B, 398 (1983).

[18] P. Bagnaia et al, Phys. Lett. B 129, 130 (1983).

[19] C. Caso et al, European Physical Journal C3, 1 (1998).

[20] V. Berger, R. Phillips, "Collider Physics", Addison-Wesley, 1987.

[21] B. Webber, "Fragmentation and Hadronization", e-conference C990809.

[22] B. Webber, P. Nason, and O. Biebel, "Jet Fragmentation in $e^{+} e^{-}$Annihilation", hep-ph/0109282, Nov 2001.

[23] L. N. Lipatov, Sov. J. Nucl. Phys. 20, 95 (1975); V.N. Gribov and L.N. Lipatov, Sov. J. Nucl. Phys. 15, 438 (1972); G. Altarelli and G. Parisi, Nucl. Phys. B126, 298 (1977); Yu.L. Dockshitzer, Sov. Phys. JETP 46, 641 (1977).

[24] D. J. Fox et al., Phys. Rev. Lett 33, 494 (1979).

[25] V.N. Gribov and L.N. Lipatov, Sov. J. Nucl. Phys. 15, 438 (1972); L.N. Lipatov, Sov. J. Nucl. Phys. 20, 94 (1975); G. Altarelli and P. Parisi, Nucl. Phys. B 126, 298 (1977); Yu. L. Dokshitzer, Sov. Phys. JETP, 46,641 (1977).

[26] Yu. L. Dokshitzer and S. L. Troyan, Proc. 19th Winter School of the LNPI, Vol. I, p. 144; A. H. Mueller, Nucl. Phys. B 213, 85 (1983).

[27] Yu. Dokshitzer, S. Troyan, XIX Winter School of LNPI, vol. 1, p. 144 (1984); A. H. Mueller, Nucl. Phys. B 213, 85 (1983).

[28] A.N. Safonov, CDF thesis - "Jet Fragmentation and Predictions of the Resummed Perturbative QCD", 2001

[29] X. Artru and G. Mennessier, Nucl. Phys. B70, 93 (1974); B. Andersson, G. Gustafson, G. Ingelman, T. Sjostrand, Phys. Reports 97, 31 (1983). 
[30] Ya. I. Azimonv, Yu. Dokshitzer, V. Khoze, and S. Troyan, Z. Phys. C 27,65 (1985).

[31] Marechesini et al., HERWIG v 5.1 a Monte Carlo generator that simulates the initial and final state radiation and hadronization, 1992.

[32] V.S. Fadin, Sov. Journ. Nucl. Phys. 37, 245 (1983).

[33] G. Marchesini et al., Comp. Phys. Comm. 67, 465 (1992); G. Corcella et al., JHEP 0101, 010 (2001).

[34] Torbjorn Sjostrand, "PYTHIA v5.7" and "JETSET 7.4" Physics and Manual, Comput. Phys. Commun. 82, 741994.

[35] CDF collaboration, "The Collider Detector at Fermilab" - A Compilation of Articles Reprinted from Nuclear Instruments and Methods in Physics Research, 1988.

[36] K. Burkett, "Trigger Tables", 1995.

[37] E. Buckley-Geer and S. Lammel, "How to Find Out What Run1B Data is Available", 1994.

[38] Anwar Ahmad Bhatti CDF note 3642

[39] Anwar Ahmad Bhatti CDF note 5286

[40] T. Daniels, CDF thesis - "Charmoniun Production in $p \bar{p}$ Collisions at $\sqrt{s}=1.8 \mathrm{TeV} 1800 \mathrm{GeV}$ ", 1997

[41] M.H. Schub, CDF thesis - "Strange Particle Production in ProtonAntiproton Collisions at Center of Mass Energies of $630 \mathrm{GeV}$ and $1800 \mathrm{GeV}, 1989$

[42] J.B. Gonzalex, J. G. Heinrich, N. S. Lockyer CDF note 3010

[43] M. Deninno, N. Moggi, F. Rimondi, CDF note 6075

[44] T. A. Keaffaber, CDF thesis - "Measurement of the $B^{+}$Meson Cross Section in Proton-Antiproton Collisions at $1.8 \mathrm{TeV}$ Using the Fully Reconstructed Decay $B^{+} \rightarrow J / \psi K^{+"}, 2000$

[45] J. Marriner CDF note 1996

[46] N. Moggi, F. Rimondi CDF note 5575

[47] S. Oh and C. Wang CDF note 4343 
[48] T. Devlin CDF note 4205

[49] P. Perchonok, fakeevent.cdf, 1995

[50] CDF collaboration, CDF detector simulator CDFSIM

[51] N. Moggi, F. Rimondi CDF note 6043

[52] F. Bedeschi and A. Mukherjee, qtrkpr.cdf, 1993

[53] A. Caner and A. Mukherjee, ctaddh.cdf, 1994

[54] M.W. Bailey, A. F. Garinkel, S. M. Tkaczyk CDF note 2815

[55] Andreas Warburton CDF note 4139

[56] Todd A. Keaffaber, Jonathan D. Lewis, Mark W. Bailey, Daniela Bortoletto, Slawek Tkaczyk, Arthur F. Garfinkel CDF note 4768

[57] A. Caner, A. Mukherjee, A. Yagil CDF note 2363

[58] William Trischuk, Andreas Warburton CDF note 4423

[59] Ting Miao CDF note 3843

[60] Petar Maksimovic, G. Bauer, J. Friedman, T. Shah, P. Sphicas, J. Tseng CDF note 4487

[61] Fumihiko Ukegaw CDF note 5204

[62] Personal Correspondence with M. Kirk, P. Maksimovic, and S. Oh on separate occassions.

[63] "Measurement of B Meson Lifetimes using Fully Reconstructed B Decays Produced in $p \bar{p}$ Collisions at $\sqrt{s}=1.8 \mathrm{TeV} ", \mathrm{D}$. Acosta et al., The CDF Collaboration, Phys. Rev. D65, 092009 (2002).

[64] "B Lifetimes, Mixing and CP Violation at CDF", M. Paulini, Int. J. Mod. Phys. A14, 2791 (1999).

[65] " $K_{S}$ production in $p \bar{p}$ interactions at $\sqrt{s}=630$ and $1800 \mathrm{GeV}$ ", F. Abe et al., The CDF Collaboration, Phys. Rev. D 40, 3791 (1989).

[66] "Topology of three-jet events in $p \bar{p}$ Collisions at $\sqrt{s}=1.8 \mathrm{TeV}$ ", Phys. Rev. D 45,1448 (2001)

[67] B. Flaugher et al CDF note 2902 
[68] N. Eddy CDF note 3534

[69] J.R. Dittmann CDF note 4001

[70] L. Galtieri et al CDF note 3253

[71] Anwar Ahmad Bhatti CDF note 4440

[72] Anwar Ahmad Bhatti CDF note 5285

[73] "Measurement of the Inclusive Jet Cross Section in $p \bar{p}$ Collisions at $\sqrt{s}=$ 1.8 TeV", Phys. Rev. D64,032001(2001)

[74] Anwar Bhatti, 'CDF note 5108

[75] Anwar Bhatti, CDF note 4678

[76] Anwar Bhatti and B. Flaugher, CDF note 4890

[77] R. Akers et al., Z. Phys. C63, 181 (1994).

[78] P. Abreau et al., Nucl. Phys. B444, 3 (1995).

[79] D. Buskulic et al., Z. Phys. C66, 355 (1995).

[80] H. Aihara et al., Phys. Rev. Lett. 61, 1263 (1988).

[81] H. Albrecht et al., Z. Phys. C44, 547 (1989).

[82] "Momentum Distribution of Charged Particles in Dijet Events in $p \bar{p}$ Collisions at $\sqrt{s}=1.8 \mathrm{TeV}$ and Comparisons to Perturbative QCD Predictions", Phys. Rev. D65,092002(2002)

[83] "Charged Particle Multiplicity in $p \bar{p}$ Collisions at $\sqrt{s}=1.8 \mathrm{TeV}$ ", Phys. Rev. Letters 87,211804(2001) the Resummed Perturbative QCD", 2001

[84] Valeria Tano CDF note 5757

[85] Anwar Bhatti, Eve Kovacs, Joey Huston, Valeria Tano CDF note 5600

[86] Anwar Bhatti, Joey Huston, Eve Kovacs, Valeria Tano CDF note 5214

[87] "Charged Jet Evolution and the Underlying Event in Proton-Antiproton Collisions at 1.8 TeV", Phys. Rev. D65,092002(2002)

[88] Rick Field CDF note 5746

[89] Rick Field CDF note 5245 
[90] CDF collaboration, CDF detector simulator QFL v3.59

$$
\sqrt{s}=1.8 \mathrm{TeV}^{\prime \prime}, 1998
$$


Proguest

2017 ProQuest Distribution Agreement

Agreement is between the author (Author) and Proquest ULC.
West/UMI) Under this Agreement, Author grants Pro quest ce

timon I. License for Inclusion of the Work in Pro Quest Publishing Program

and
Dr.

Dance university 